\title{
Cultural Differences in the Autobiographical Memory of Trauma
}

\author{
Laura A. Jobson
}

A thesis submitted for the degree of Doctor of Philosophy (in Clinical Psychology) from the Australian National University

School of Psychology, ANU, Canberra, Australia

January, 2008 



\section{DECLARATION}

I declare that this thesis reports my original work, that no part has been previously accepted and presented for the award of any degree or diploma from any university, and that, to the best of my knowledge, no material previously published or written by any other person is included, except where due acknowledgement is given.

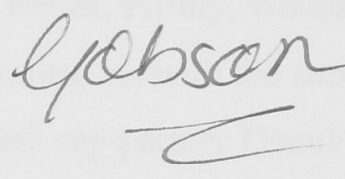

Laura A. Jobson 


\section{DEDICATION}

This thesis is dedicated to the trauma survivors that participated in this research. Their resilience, strength and courage are admired. Additionally, the thesis is dedicated to the advancement of cross-cultural clinical psychology and to the day in which non-Western clinical psychology is as prevalent in our research as that of Western clinical psychology. 


\section{ACKNOWLEDGEMENTS}

Firstly, I would like to thank and acknowledge my supervisor Associate Professor Richard O'Kearney. His assistance, direction, support and encouragement were essential in the production of this thesis. Secondly, I would like thank and acknowledge my supervisory panel, Professor Marie Carroll and Dr Ross Wilkinson, for their guidance, direction, assistance and knowledge. Thirdly, I acknowledge Zhong Tao for his assistance with coding. Fourthly, I would like to acknowledge the trauma survivors that participated in this research. I would like to thank them for the courage they demonstrated by participating in this research. The participants retrieved memories and discussed very personal issues. Fifthly, would like to acknowledge and thank the Australian National University for providing me with the support to complete the $\mathrm{PhD}$. Finally and most importantly, I would like to thank my partner Danny for his support, patience, humor and long-suffering, my parents Phillip and Anne, my siblings Peter and Amy, my grandparents and extended family for their continual support and encouragement, and my colleagues for making the journey an adventure. 


\section{PUBLICATIONS ARISING FROM THIS THESIS}

It is acknowledged that the findings of this thesis have contributed to the following articles:

Jobson, L., \& O’ Kearney, R.T. (2006). Cultural differences in the autobiographical memory of trauma. Clinical Psychologist, 10, 89-98.

Jobson, L., \& O'Kearney, R.T. (in press). Cultural differences in the retrieval of self-defining memories. Journal of Cross-Cultural Psychology.

Jobson, L., \& O’Kearney, R.T., (in press). Cultural differences in personal identity in posttraumatic stress disorder. British Journal of Clinical Psychology. 


\section{ABSTRACT}

This thesis explored cultural differences in autobiographical memory of trauma. Cultural differences in self-construal impact on the psychological processes (e.g., autobiographical memory, appraisals, schema, etc.) which have been implicated by current posttraumatic stress disorder (PTSD) models in the etiology and maintenance of PTSD. However, at present, models of PTSD have not considered cultural differences in self-construal. The objectives of the thesis were to investigate the impact of cultural variation in self-construal on (a) autobiographical remembering of trauma, (b) integration of the trauma memory, (c) appraisals, and (d) meaning and belief systems. The thesis was comprised of two series of studies. Series 1 explored the objectives in a non-clinical sample. Series 2 examined the objectives in a clinically relevant sample of trauma survivors with and without PTSD. The methodology adopted a questionnaire approach that investigated autobiographical remembering of trauma using narratives and selfdefining memories; integration of the trauma memory using personal goals, self-defining memories and self-cognitions; appraisals using trauma narratives; and meaning and belief systems using free-response questions. First, the thesis found while the cultural distinction between autonomy versus relatedness was evident in autobiographical memories of everyday events, no cultural distinction was evident in the autobiographical remembering of trauma. The autobiographical remembering of trauma was predominately autonomous. Moreover, it was found that the cultural distinction in self impacts on the relationship between the nature of autobiographical remembering and posttraumatic psychological adjustment. Trauma survivors from independent cultures with PTSD had less autonomous orientation in their autobiographical remembering than trauma survivors from independent cultures without PTSD. In contrast, trauma survivors from interdependent cultures with PTSD had more autonomous orientation in their autobiographical remembering than trauma survivors from interdependent cultures without PTSD. Second, the thesis demonstrated that the cultural distinction in self moderated trauma's impact on personal identity, self-definition and psychological adjustment to trauma. Traumaexposed participants from independent cultures with PTSD clearly displayed a more traumadefined personal identity than those without PTSD. For those from interdependent cultures, however, there were no differences in trauma-centered identity between the PTSD and no PTSD groups. Third, it was found that the cultural difference in self moderates the relationship between 
cognitive appraisal styles and psychological adjustment. Specifically, for independent cultures those with PTSD had more negative autonomous appraisals and more alienation appraisals in their trauma memories than those without PTSD. However, those with and without PTSD from interdependent cultures did not differ regarding autonomous cognitive appraisal styles but rather trauma survivors from interdependent cultures with PTSD had more alienation appraisals in their trauma narratives than trauma survivors from interdependent cultures without PTSD. Finally, it was shown that cultural variation in self impacts on the meanings attached to the trauma autobiographical memory; trauma survivors from independent cultures focused on self-change and life-change, while trauma survivors from interdependent cultures focused on interdependence. Theoretically, PTSD models were challenged to articulate how the cultural self aligns with their accounts of PTSD and a model, 'Threat to the Conceptual Self' model, was developed to account for the relationship between trauma and the conceptual self in the etiology and maintenance of PTSD. Clinically, it was emphasized that cognitive behavior therapy for PTSD is expanded to include public and communal aspects of the self. Limitations and future directions were considered. 


\section{TABLE OF CONTENTS}

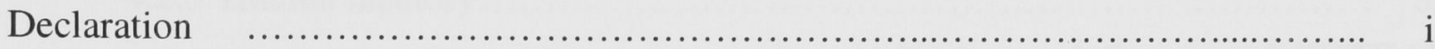

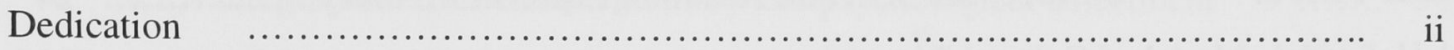

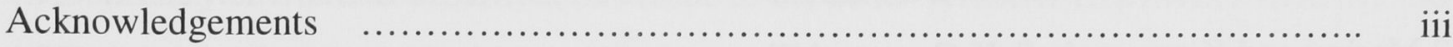

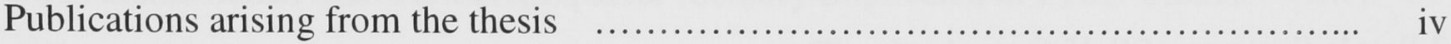

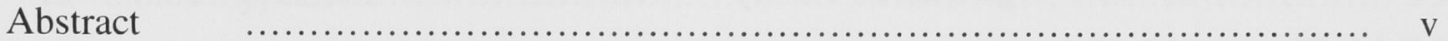

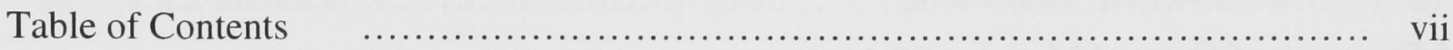

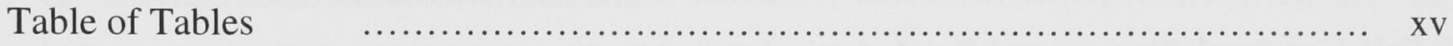

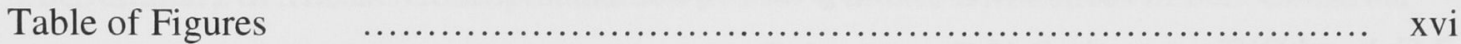

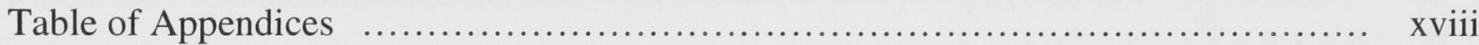

CHAPTER 1. Overview $\quad$.............................................. 1

CHAPTER 2. Socio-cognitive models of Posttraumatic Stress Disorder ..... 5

2.1 Definition of Posttraumatic Stress Disorder (PTSD) $\quad$....................... 5

2.2 History of the Emergence of Autobiographical Memory in PTSD Models $\quad \ldots . . \quad 5$

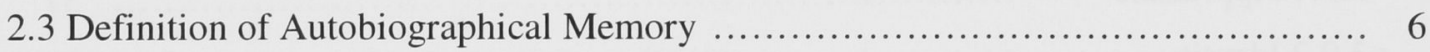

2.4 Content, Structure and Function of Autobiographical Memory $\ldots \ldots \ldots \ldots \ldots \ldots \ldots \ldots$

2.4.1 Social function $\ldots \ldots \ldots \ldots \ldots \ldots \ldots \ldots \ldots \ldots \ldots \ldots \ldots \ldots \ldots \ldots \ldots \ldots \ldots \ldots \ldots \ldots, \quad 8$

2.4.2 Directive function $\quad \ldots \ldots \ldots \ldots \ldots \ldots \ldots \ldots \ldots \ldots \ldots \ldots \ldots \ldots \ldots \ldots \ldots \ldots \ldots \ldots, \quad 8$

2.4.3 Self function $\quad$................................................... 8

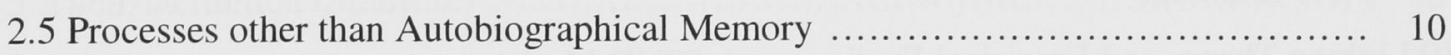

2.6 Socio-Cognitive Models of PTSD $\quad$................................ 10

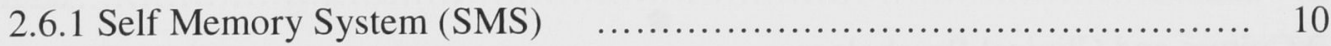

2.6.2 Appraisal model $\ldots \ldots \ldots \ldots \ldots \ldots \ldots \ldots \ldots \ldots \ldots \ldots \ldots \ldots \ldots \ldots \ldots \ldots \ldots \ldots, 14$

2.6.3 The schematic, propositional, analogue and associative representational systems

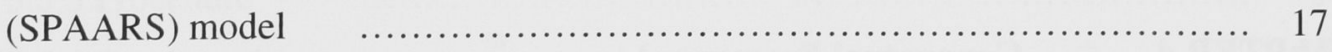

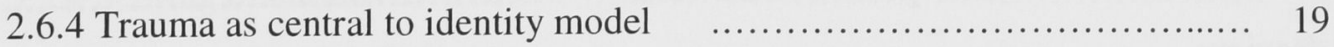

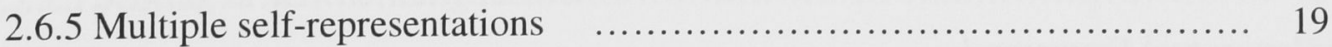

2.7 Summary of PTSD Models and their Current Links to the Self $\ldots \ldots \ldots \ldots \ldots \ldots \ldots \ldots \ldots$

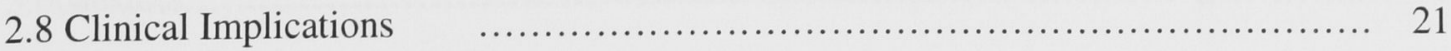


CHAPTER 3. Culture, the Self, and Autobiographical Memory .............. 24

3.1 Hofstede's Model of Cultural Dimensions $\quad$.............................. 24

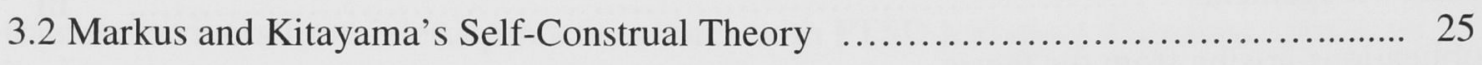

3.3 Sato's Systems of Self-Organization Theory $\quad$............................ 27

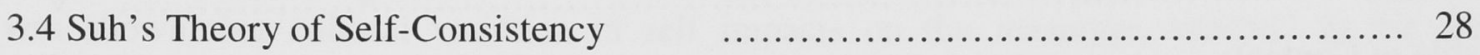

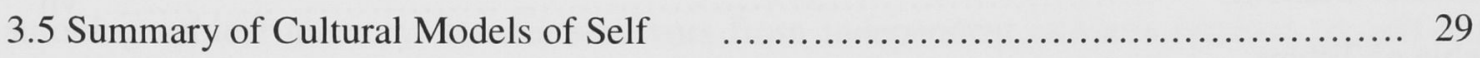

3.6 Self-Construal, Trauma and Psychological Adjustment ...................... 30

3.7 Cultural Differences in Self-Construal Influence on Autobiographical Memory 30

3.7.1 Social cultural development theory of autobiographical memory $\quad \ldots \ldots \ldots . .31$

3.8 Linking Theoretical Stances and Empirical Findings of Cultural Variations in

Autobiographical Memory with PTSD Models $\quad$.............................. 35

3.9 Cultural Differences in Self-Construal Influence on other Processes Implicated in PTSD

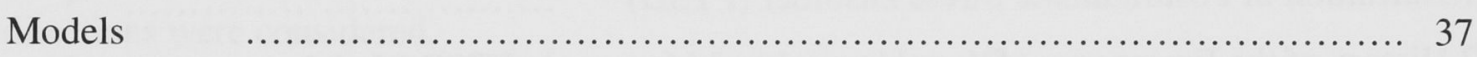

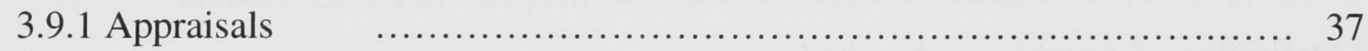

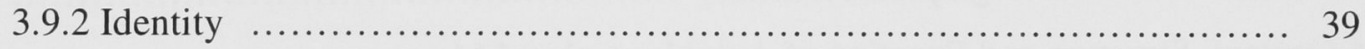

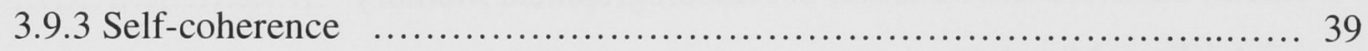

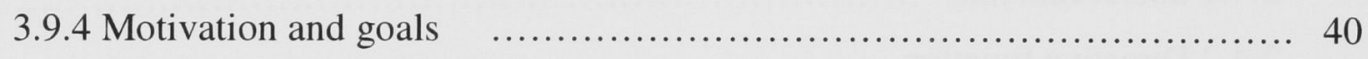

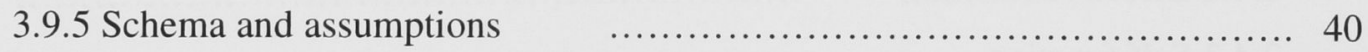

3.9.6 Emotions $\quad$............................................... 41

3.10 Linking Theory and Empirical Findings with PTSD Models $\quad \ldots \ldots \ldots \ldots \ldots \ldots \ldots . . \ldots 1$

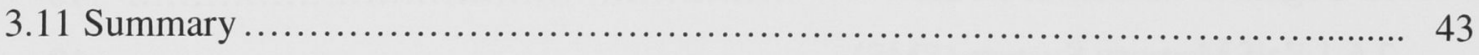

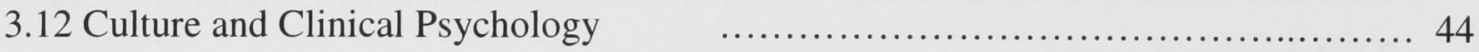

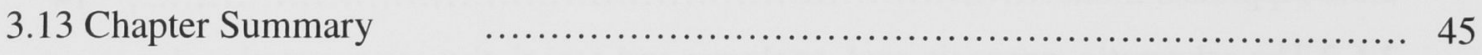

CHAPTER 4. Conceptual Framework $\quad$................................... 46

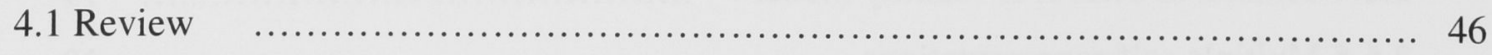

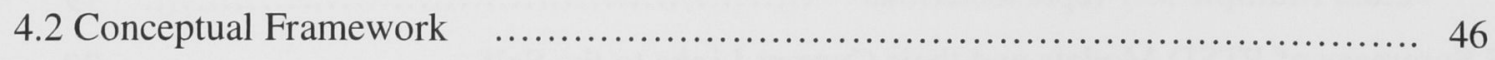

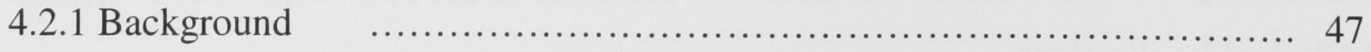




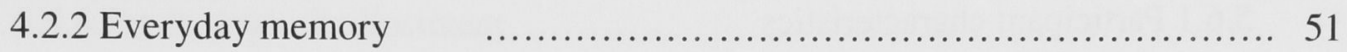

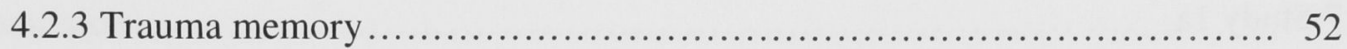

4.2.4 Integration of the trauma memory and psychological adjustment $\quad \ldots . . .54$

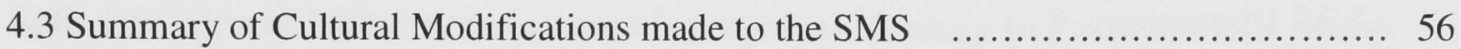

4.4 Processes other than Autobiographical Memory Implicated in PTSD Models ..... 59

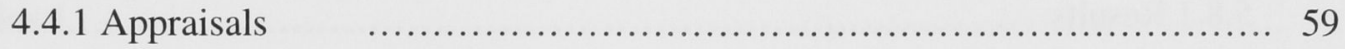

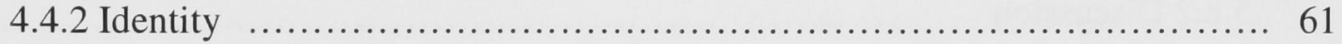

4.4.3 Transformation of meaning $\quad$........................................ 62

4.5 Summary of Thesis' Conceptualization of how Cultural Differences in Self-Construal Moderate Processes other than Autobiographical Memory Implicated in PTSD Models 63

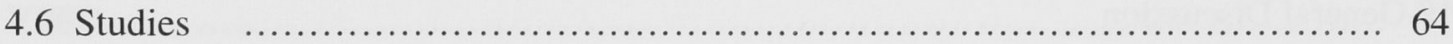

4.7 Conclusion $\quad$........................................................... 66

SERIES 1 CULTURAL DIFFERENCES IN AUTOBIOGRAPHICAL MEMORY OF TRAUMA IN A NON-CLINICAL SAMPLE $\quad$................................ xix

Chapter 5. Study 1: Cultural Differences in Autobiographical Memory of Trauma and Retrieval of Self-Defining Memories in a Non-Clinical Sample $\ldots . . .67$

5.1 Autobiographical Remembering of Everyday and Trauma Events (Study 1a) ..... 67

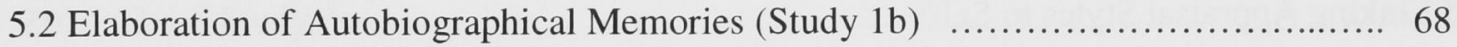

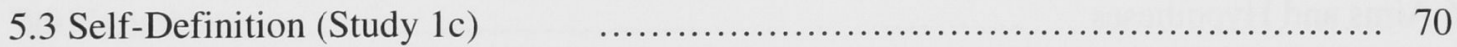

5.4 Hypotheses $\quad$........................................................... 72

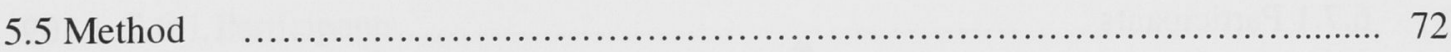

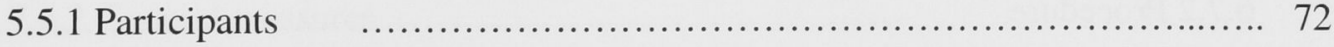

5.5.2 Measures $\quad$.

5.5.3 Procedure $\quad$................................................... 74

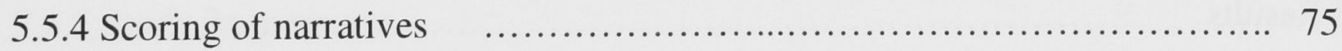

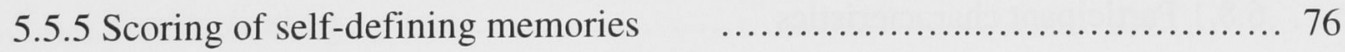

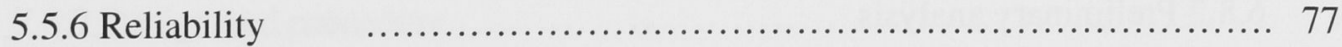

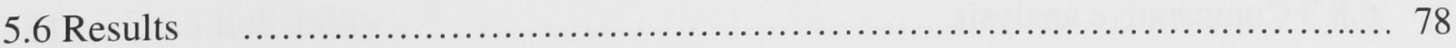


5.6.1 Participant characteristics $\quad$....................................... 78

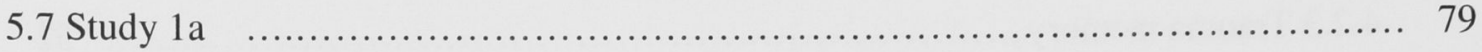

5.7.1 Results ........................................................... 79

5.7.2 Discussion $\quad$...................................................... 84

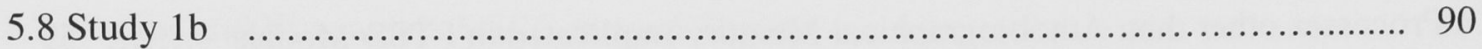

5.8.1 Results ............................................................ 90

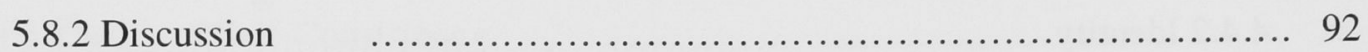

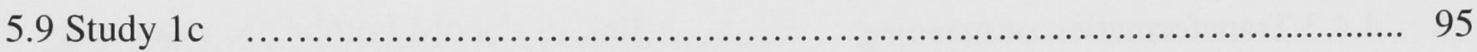

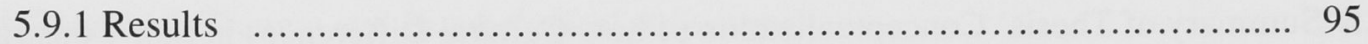

5.9.2 Discussion $\quad$...................................................... 96

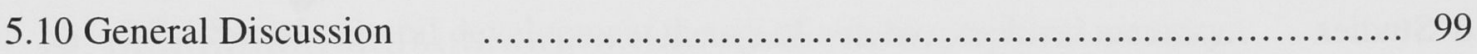

CHAPTER 6. Study 2: Cultural Differences in Cognitive Appraisal Styles Utilized During Trauma and Subsequent Impact on Psychological Adjustment in a Non-Clinical Sample $\quad$.................................................................... 102

6.1 Mental Defeat/Lack of Psychological Autonomy $\quad$.......................... 103

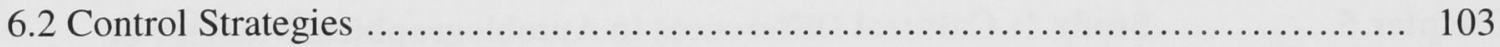

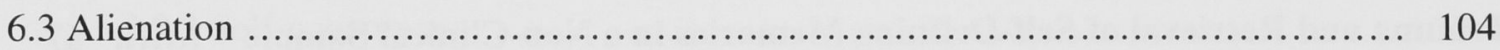

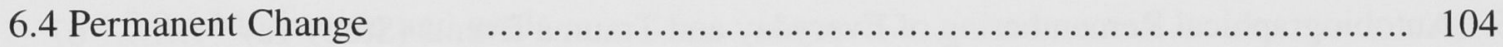

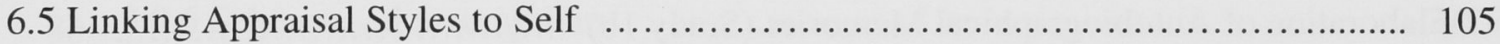

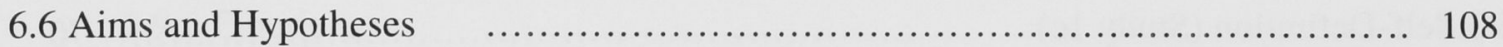

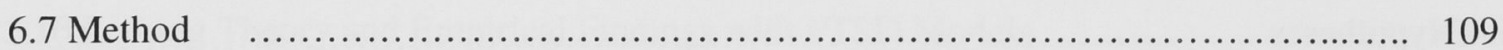

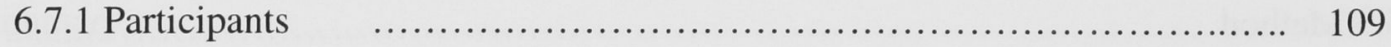

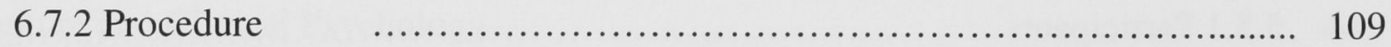

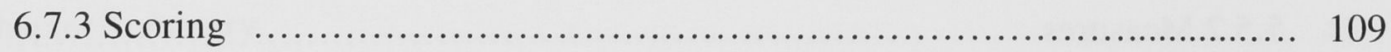

6.7.4 Reliability $\quad$...................................................... 112

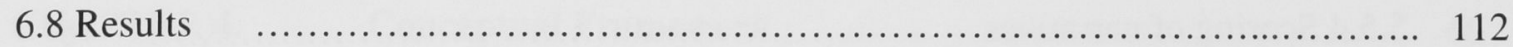

6.8.1 Participant characteristics $\quad$........................................ 112

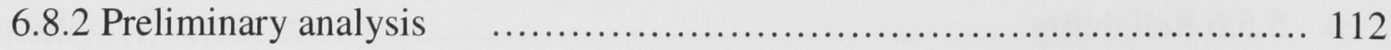

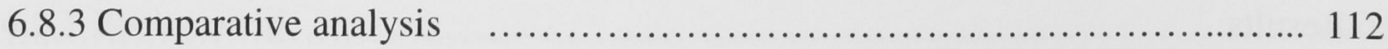


6.8.4 Psychological adjustment $\quad$.......................................... 113

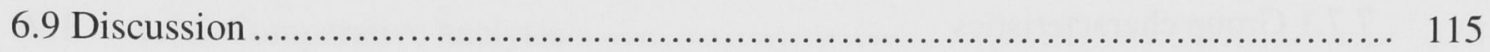

Series 2 Cultural Differences in Autobiographical Memory in Posttraumatic Stress

Disorder: Extending the Ecological Validity, and Theoretical and Clinical Implications of Series 1

CHAPTER 7 Study 3: Cultural Differences in Autobiographical Memory of Trauma and Personal Identity in Posttraumatic Stress Disorder

7.1 Relationship between Cultural Differences in Autobiographical Memory and

PTSD

7.1.1 Centrality of the trauma memory

7.2 Threat to the Conceptual Self

7.2.1 Impact on the functional role of autobiographical memory

7.2.2 Impact on other autobiographical memories

7.3 Trauma Central to Identity

7.4 Sub-Studies

7.4.1 Study $3 \mathrm{a}$

7.4.2 Study $3 b$

7.4.3 Study $3 \mathrm{c}$

7.5 Hypotheses

7.6 Method

7.6.1 Participants

7.6.2 General measures

7.6.3 Study 3 a measures

7.6.4 Study $3 \mathrm{~b}$ measures

7.6.5 Study $3 c$ measures

7.6.6 Scoring/coding system

7.6.7 Design and procedure

7.6.8 Reliability 
7.7 Results

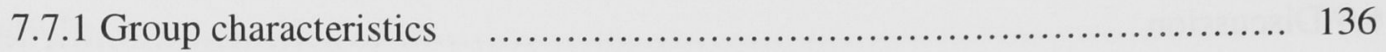

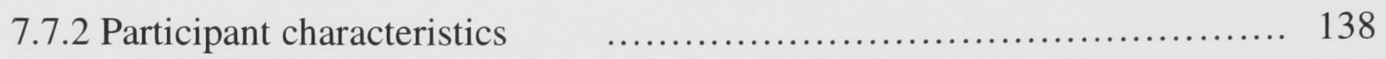

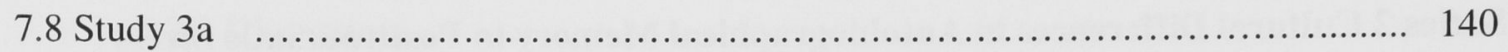

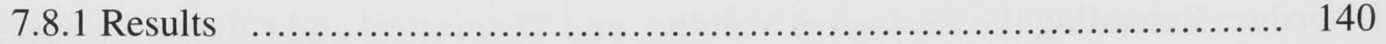

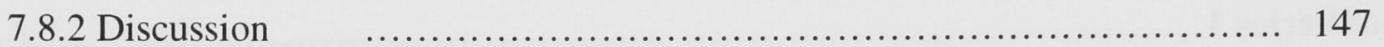

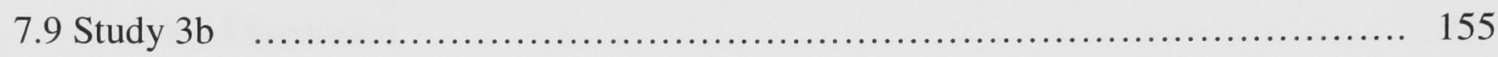

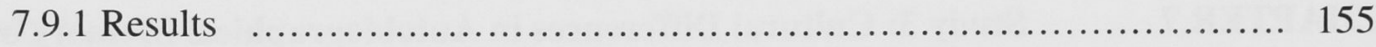

7.9.2 Discussion $\quad$............................................... 158

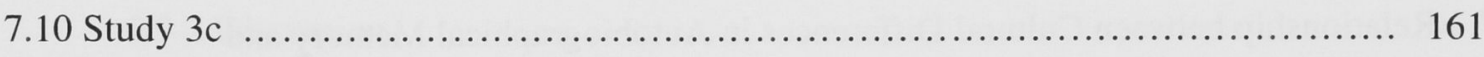

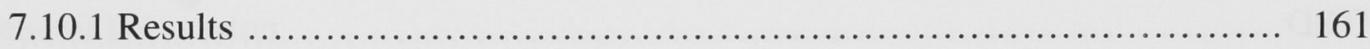

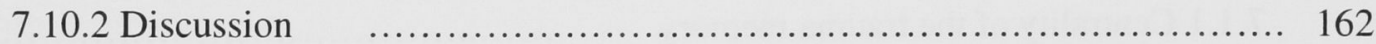

7.11 General Discussion $\quad$............................................... 164

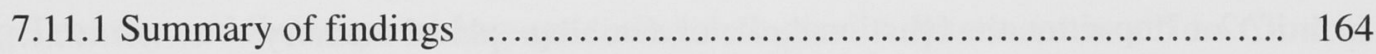

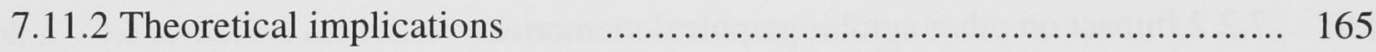

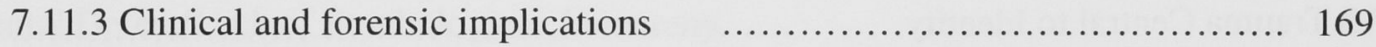

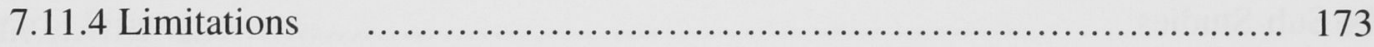

7.11 .5 Conclusion $\quad$.............................................. 174

CHAPTER 8 Study 4: Cultural Differences in Cognitive Appraisal Styles Utilized During Trauma in those with and without PTSD .............................. 176

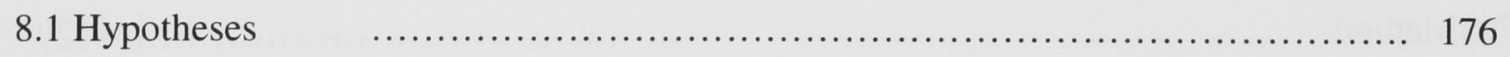

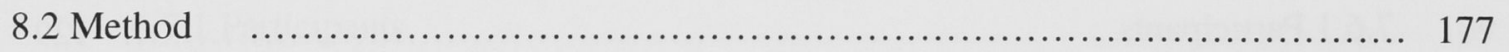

8.2.1 Participants $\quad$................................................ 177

8.2.2 Procedure $\quad$................................................. 177

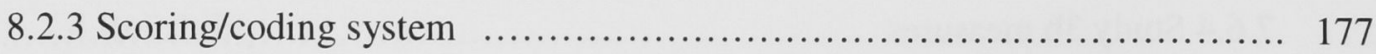

8.2.4 Reliability $\quad$................................................ 177

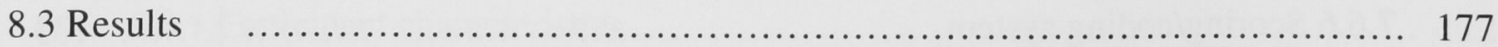

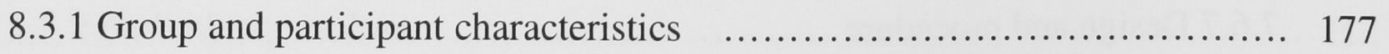


8.3.2 Preliminary analysis _............................................... 178

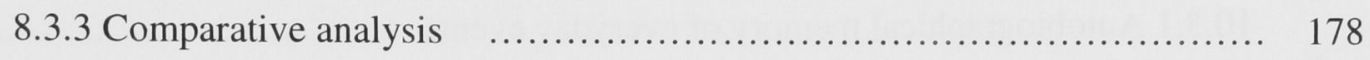

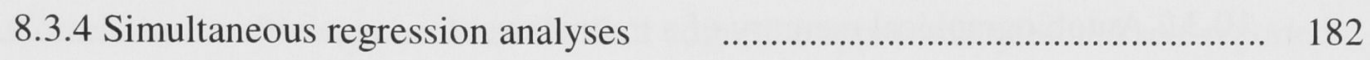

8.3.5 Trauma type (interpersonal versus non-interpersonal) .................. 183

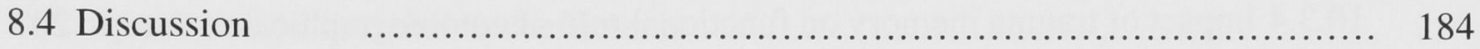

\section{CHAPTER 9 Study 5: Cultural Differences in Meanings Attached to Trauma}

Autobiographical Memory in Posttraumatic Stress Disorder $\quad$.................................. 190

9.1 Hypotheses

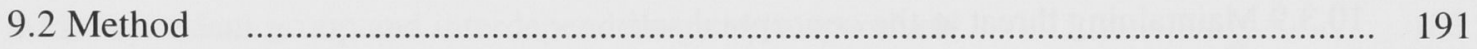

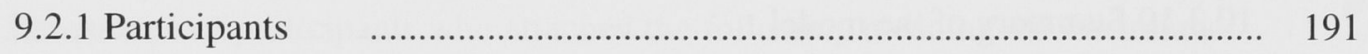

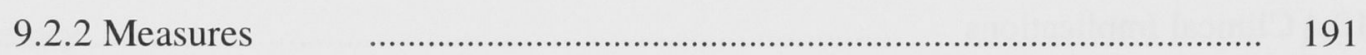

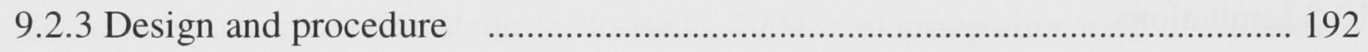

9.3 Results

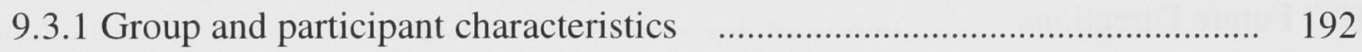

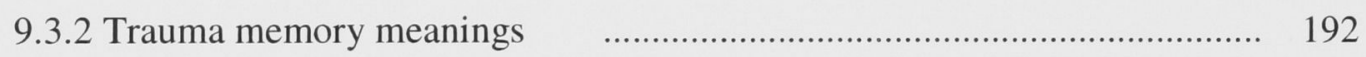

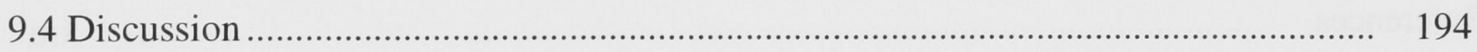

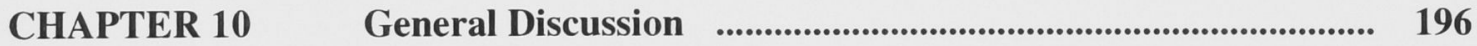

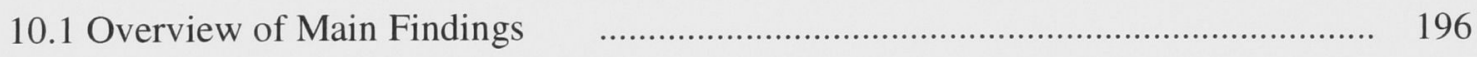

10.1.1 Autobiographical remembering of trauma _....................................... 196

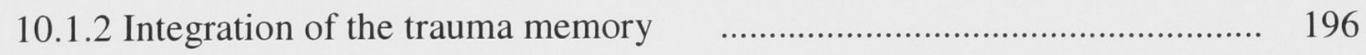

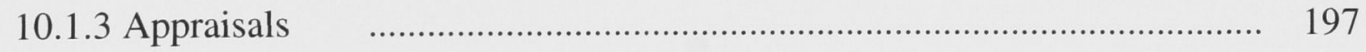

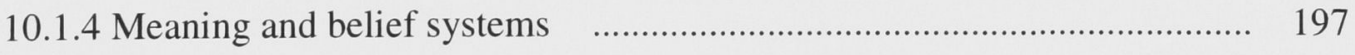

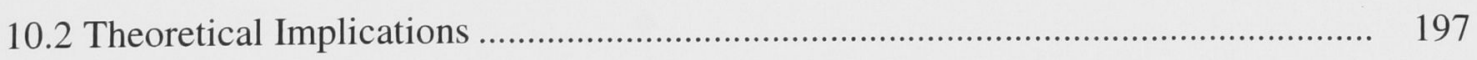

10.2.1 Self-memory system (SMS) _......................................................... 198

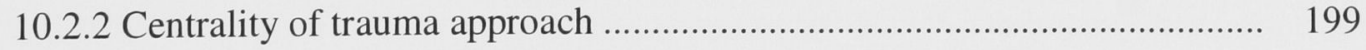

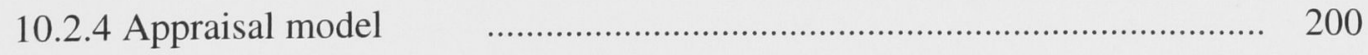

10.2.5 Transformation of meaning models _............................................... 201

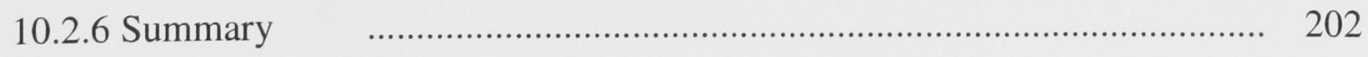


10.3 'Threat to Conceptual Self' (TCS) Model $\quad$................................................... 203

10.3.1 Autobiographical memory of everyday events $\quad$............................ 205

10.3.2 Autobiographical memory of a trauma event .................................... 205

10.3.3 Centrality of the trauma memory $\quad$............................................... 206

10.3.4 Impact of trauma memory on functional role of autobiographical memory 207

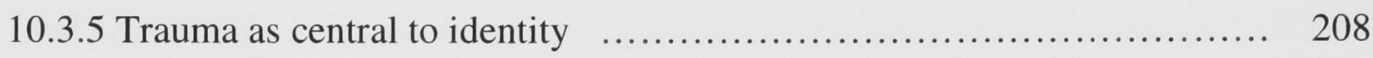

10.3.6 Appraisals $\quad$.............................................. 209

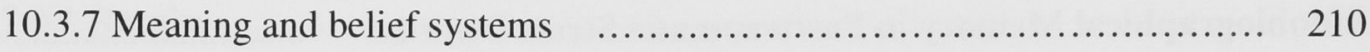

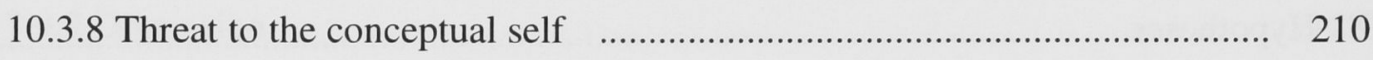

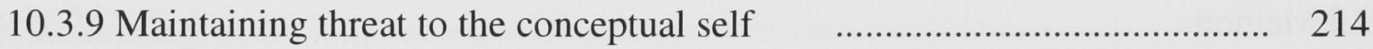

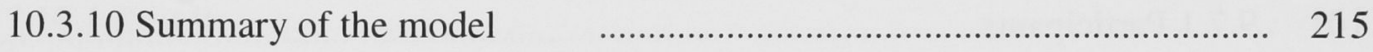

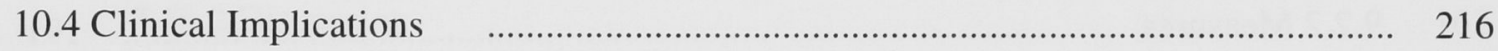

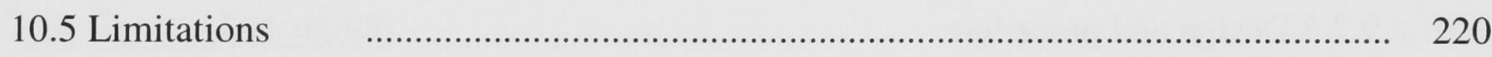

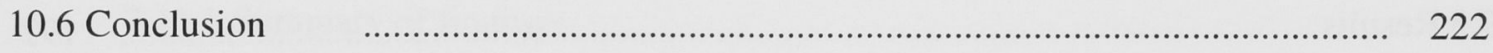

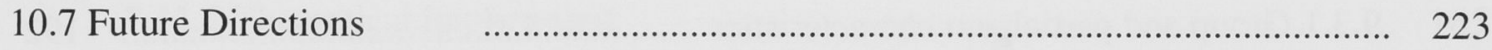

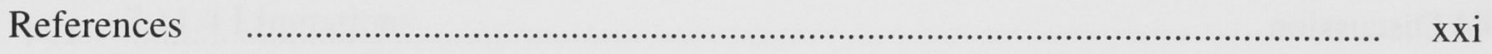

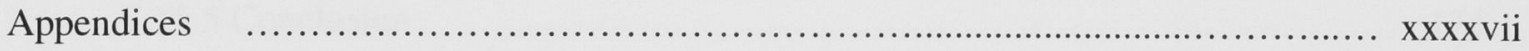




\section{TABLE OF TABLES}

6.1 Correlation coefficients between the four cognitive appraisal styles and self-reported adjustment to trauma for the Asian and Australian group and $Z$ difference scores between

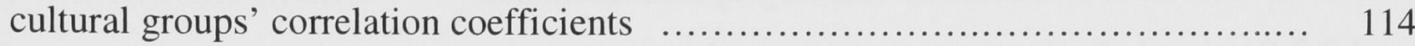

7.1 Means and (standard deviations) of group characteristics $\quad \ldots \ldots \ldots \ldots \ldots \ldots \ldots \ldots \ldots \ldots$

7.2 Means and (standard deviations) of variables scored in the self-defining memories

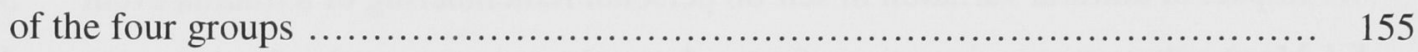

8.1 Summary of simultaneous regression analysis for variables predicting PTSD for the independent group and interdependent group respectively

9.1 Percentage of participants who attached the following meanings to the trauma memory

A1. Individualism scores (Hofstede \& Hofstede, 2004) and group allocation of cultures used in thesis xxvii 


\section{TABLE OF FIGURES}

4.1 Knowledge structures in autobiographical memory $\ldots \ldots \ldots \ldots \ldots \ldots \ldots \ldots \ldots \ldots \ldots$

4.2.1 Retrieval of autonomous autobiographical memories $\quad$................... 50

4.2.2 Retrieval of relatedness autobiographical memories $\quad$................... 50

4.3 Impact of cultural variation in self on personal remembering of an everyday event 52

4.4 Impact of cultural variation in self on personal remembering of a trauma event 53

5.1 Mean autonomous orientation of everyday and trauma memories for Asian international students and Australian domestic students

5.2 Mean other/self ratio of everyday and trauma memories for Asian international students and Australian domestic students

5.3 Mean reflective comment of everyday and trauma memories for Asian international students and Australian domestic students

5.4 Mean number of units of elaboration in memories for each group by theme of memory

7.1 Relations between culture, self and memory

7.2 The adjusted mean autonomous orientation for the independent PTSD group, independent no PTSD group, interdependent PTSD group and interdependent no PTSD groups for both the everyday and trauma memory

7.3 The adjusted mean reflective comments for the independent PTSD group, independent no PTSD group, interdependent PTSD group and interdependent no PTSD groups for both the everyday and trauma memory.....

7.4 Adjusted means for the trauma ratios for goals, self-defining memories and selfcognitions

8.1 Adjusted means of mental defeat for independent and interdependent groups with and without PTSD

8.2 Adjusted means for control strategies of independent and interdependent culture group with and without PTSD

8.3 Adjusted means for alienation of independent and interdependent groups with and without PTSD 
8.4 Adjusted means for permanent change of independent and interdependent group with and without PTSD 182

10.1 Threat to Conceptual Self Model 204 
TABLE OF APPENDICES
A. Individualism Scores
xxxvii
B. Additional Statistical Analyses for Study 4
xxxix 


\title{
CHAPTER 1
}

\section{Overview}

\begin{abstract}
People of different religions and cultures live side by side in almost every part of the world, and most of us have overlapping identities which unite us with very different groups. We can love what we are, without hating what - and who - we are not. We can thrive in our own tradition, even as we learn from others, and come to respect their teachings.
\end{abstract}

-Kofi Annan, 2001, Former Secretary-General of the United Nations, Nobel Lecture

This thesis investigated cultural differences in the autobiographical memory of trauma. In recent years some of the most prominent theoretical models of psychological adjustment following trauma have focused on the role of autobiographical memory in distinguishing between those with adaptive adjustment from those with disrupted adjustment. Simultaneously, theory positing cultural differences in self-construal has been applied to autobiographical memory research. Such research has continuously demonstrated the impact of cultural variation in the self on autobiographical memory. Despite current models of posttraumatic stress disorder (PTSD) representing impressive advances in the understanding of the etiology, maintenance and treatment of disrupted adjustment following trauma, these models have been developed independently of recent findings regarding the importance of cultural variation in autobiographical memory.

PTSD, characterized by symptoms of repeated and unwanted re-experiencing of the event, hyperarousal, emotional numbing and avoidance (Diagnostic and Statistical Manual of Mental Disorders-IV; American Psychiatric Association, 1994), is affiliated with "disturbances in a wide range of psychological processes including memory, attention, cognitive-affective reactions, beliefs, coping strategies, and social support" (Brewin \& Holmes, 2003, p. 345). However, Brewin and Holmes (2003, p. 345) claim the psychological processes "most likely unique to PTSD, compared to other psychological disorders, are the unusual and inconsistent memory phenomena". This, alongside the personal significance of the trauma, has resulted in researchers increasingly concentrating on the role of autobiographical memory in the understanding of PTSD. 
Current PTSD models have been very influential both theoretically and clinically. There are two separate but related issues, however, which represent significant limitations to these formulations and their broader clinical applicability. The first is that the majority of these models do not take up the challenge of fitting their propositions about autobiographical memory with psychological understandings of the nature of self. Perhaps as a consequence, they ignore the second issue. This is the impact of culture on the unique, intrinsic, and symbiotic relationship between the self and autobiographical memory (e.g., Conway \& Holmes, 2004; Wang \& Conway, 2004). Because culture is critical to the nature and development of the self, it is also needs to become a critical issue in research in adjustment to trauma.

One important cultural distinction that influences the nature and development of the self is the distinction between an autonomous/independent (an emphasis on the unitary and stable entity of the individual, a promotion of private aspects of self and individual goals, and an aim to be autonomous, self-reliant and unique) versus a relatedness/interdependent (an emphasis on interdependence, connection with the social context and group harmony) orientation (Kitayama, Markus, Matsumoto, \& Norasakkunkit, 1997; Triandis, 1995). Cultural theorists (e.g., Markus \& Kitayama, 1991; Sato, 2001) espouse that although all individuals possess both autonomous and relatedness aspects of self, collectivistic cultures (generally non-Western cultures) give emphasis to the dominance of an interdependent self, while individualistic cultures (generally Western cultures) tend to emphasize the dominance of an independent self (Markus \& Kitayama, 1991). This cultural distinction has been found to impact on the relationship between the self and autobiographical memory, in that the self is functional in "encoding, organizing and retrieving autobiographical memories", while autobiographical memory has a role in "developing, expressing and maintaining" (Wang \& Conway, 2004, p. 912) the self. Wang and Conway (2004) claim that by "taking different forms and contents autobiographical memories may sustain and regulate modes of selfhood endorsed by particular cultures" (p. 913).

In sum, given research has found that cultural differences in self impact on autobiographical remembering, and the centrality of autobiographical memory in our current socio-cognitive models of PTSD, an exploration into cultural differences in autobiographical memory of trauma is needed. As they stand, due to this neglect of considerations of the self, it is believed that our current models of PTSD may be limited, both theoretically and clinically. The aim of this thesis is not to disregard or 
discredit the socio-cognitive models of PTSD. These models are sophisticated, internally sound models which account for much of the phenomena observed in the development and maintenance of PTSD and have guided, in many cases successfully, clinical practice. Furthermore, the processes and concepts (such as autobiographical memory, appraisals, identity, belief systems, schema, etc.), posited to be instrumental in the etiology and maintenance of PTSD, have strong associations with the self. The objective of this thesis is to utilize these existing links with the self, to initiate a consideration of current understandings of self and most importantly to draw these models into the cultural realm.

In light of this, in order to explore cultural differences in autobiographical memory of trauma, and more generally, to examine the cultural appropriateness of our current PTSD models, this thesis considers four specific research questions related to the following themes.

- Autobiographical remembering of trauma: How might the cultural distinction in self impact on autobiographical remembering for a traumatic event?

- Integration of the trauma memory: How might the cultural distinction in self moderate trauma's impact on personal identity, self-definition and psychological adjustment to trauma?

- Appraisals: How might the cultural distinction in self impact on trauma appraisals and subsequent impact on psychological adjustment?

- Meaning and belief systems: How might the cultural variation in self impact on trauma meaning?

Two series of studies have been developed to investigate these questions. First, the questions will be researched in terms of the trauma memory. Therefore, the first series of studies will explore the impact of cultural variation in self-construal on autobiographical memory of trauma, integration of the trauma memory and appraisals in a non-clinical sample. The second series of studies will extend the ecological validity and theoretical and clinical implications of the first series of studies by exploring the impact of cultural variation in self-construal on autobiographical memory of trauma, integration of the trauma memory, appraisals, and meaning and belief systems in a clinically relevant sample of trauma survivors with and without PTSD. 
The objectives of the following chapters in this introduction are fourfold. First, there will be a chapter outlining the current socio-cognitive models of PTSD. These models emphasize the importance of autobiographical memory in distinguishing between those with and without PTSD. Second, Chapter 3 will outline how autobiographical memory serves a cultural function in terms of sustaining and regulating the self affirmed by different cultures. There will be an examination of the literature that has repeatedly demonstrated an impact of culture on autobiographical memory and other processes thought to be involved in the development and maintenance of PTSD. Third, these two areas of research will be united in the construction of a theoretical framework that accounts for cultural differences in autobiographical memory of trauma. This will provide the scaffolding for the studies developed in this thesis. Finally, the introduction will outline the studies developed in this thesis to investigate various hypotheses pertaining to the general topic of cultural differences in autobiographical memory of trauma and specifically, the four research questions outlined above. 


\section{CHAPTER 2}

\section{Socio-Cognitive Models of Posttraumatic Stress Disorder}

\subsection{Definition of Posttraumatic Stress Disorder (PTSD)}

In 1980, PTSD was first introduced into the DSM-III (1980). For a diagnosis of PTSD to be given, the person must have been exposed to a "traumatic event in which both of the following were present: (1) the person experienced, witnessed, or was confronted with an event or events that involved actual or threatened death or serious injury, or a threat to the physical integrity of others (2) the person's response involved intense fear, helplessness, or horror" (DSM-IV, 1994, pp. 427-428). The survivor must also be experiencing a range of persistent, repeated and unwanted reexperiencing of the traumatic event (i.e., flashbacks, dreams/nightmares, re-living symptoms, and emotional and physical reactions when reminded of the trauma), persistent avoidance of stimuli associated with the trauma and numbing of general responsiveness (i.e., avoidance of conversations, thoughts, activities and places that are reminders of the event, not being able to remember important aspects of the trauma, lack of interest in activities, feeling distant from others, feeling emotionally numb and a sense of foreshortened future) and persistent hyperarousal symptoms (i.e., trouble sleeping, trouble concentrating, irritability, outbursts of anger, hypervigilance and exaggerated startle response) (DSM-IV, 1994).

\subsection{History of the Emergence of Autobiographical Memory in PTSD Models}

As researchers in the 1970 s began to study rape survivors and Vietnam veterans, learning theory was drawn upon to explain the symptoms they were observing (Resick \& Calhoun, 2001). Although learning theory can account for much of the development and maintenance of the fear and avoidance symptoms seen in PTSD, it faces difficulties when attempting to explain the re-experiencing symptoms. Hence, memory needed to be included in models of PTSD and thus, socio-cognitive theories of PTSD were emphasized given their focus on memory, informationprocessing, appraisals, and the impact of the trauma on belief systems (Resick \& Calhoun, 2001). 
Consequently, given the personal significance of the trauma memory, the nature of autobiographical memory for traumatic events became an important focus of PTSD models and research. Furthermore, as outlined in greater depth below, autobiographical memory appears to have become central to PTSD models because autobiographical memory is thought to be central in allowing individuals to have and maintain an identity and coherent self-concept across the lifespan, even in the face of change and specific life-events (Bluck, Alea, Habermas, \& Rubin, 2005). Therefore, autobiographical memories are thought to be especially important when the self is in adverse conditions that require self-change (Robinson, 1986).

Current socio-cognitive models explaining the phenomena of PTSD vary in significant ways in how they conceptualize autobiographical memory and in their understanding of how trauma impacts on autobiographical memory. However, they agree in proposing that autobiographical memory in people with disturbed psychological adjustment shows particular types of "disruption" and "dysregulation". Some of the models hypothesize that those with PTSD have structural disruption in autobiographical memory such as the dominance of sensory, perceptual and emotional impressions and deficits in conceptual connections or organization of the memory, resulting in memory fragmentation, disorganization, dysregulation and intrusions (e.g., Conway \& Pleydell-Pearce, 2000). Others posit that autobiographical memories of those with PTSD are less integrated with the rest of the person's autobiographical memory and that their attempts to make sense of the event "with respect to oneself" and in relationship "to other autobiographical information" are significantly impaired (e.g., Halligan, Michael, Clark, \& Ehlers, 2003, p. 422). Other models propose that the trauma memory provides the pivotal, reference point for other autobiographical memories and self-identity (e.g., Berntsen \& Rubin, 2006, 2007).

\subsection{Definition of Autobiographical Memory}

Autobiographical memory is defined as an "explicit autobiographical memory of an event that occurred in a specific time and place in one's personal past" (Nelson \& Fivush, 2004, p. 486). Brewer (1986) argued that autobiographical memory is best conceived as the subset of human memory related to the self. Autobiographical memory is believed to "constitute a major crossroads in human cognition where considerations relating to the self, emotion, goals and personal meanings intersect" 
(Conway \& Rubin, 1993, p.103). Autobiographical memory is posited to be one's memory of self-knowledge and hence, also includes semantic and episodic knowledge about the self. It is of "fundamental significance for the self, for emotions, and for the experience of personhood, that is, for the experience of enduring as an individual, in a culture, over time" (Conway \& Pleydell-Pearce, 2000, p. 261).

Some theorists (e.g., Shallice, 1988) espouse that autobiographical memory is a distinct, identifiable memory system (Conway, 1990). However, others (e.g., Conway, 1990) are not convinced of this, given that different types of knowledge in autobiographical memory are difficult to classify. For example, is remembering to write a chapter of this thesis the same as remembering one's first day at school, and is this the same as remembering the experience of learning to ride a bike? Hence, it appears more suitable to propose autobiographical memory not as a separate and distinct type of memory but rather that autobiographical memories have "certain distinguishing characteristics which they share with other types of knowledge and memories, and that it is the pattern of these characteristics rather than any single unique feature" which identifies an autobiographical memory (Conway, 1990, p. 1). Conway (1990) claims that autobiographical memory contains self-reference; is associated with the experience of remembering, that is, there is autonoetic awareness (an awareness of the self in the past); is accompanied by interpretation and is thus, meaningful to self; and can contain context-specific information, sensory-perceptual attributions and imagery.

\subsection{Content, Structure and Function of Autobiographical Memory}

Woike, Gershkovich, Piorkowski, and Polo (1999) posit that the content of autobiographical memory refers to the theme of the memory and reveals the individual's most important concerns, while the structure of autobiographical memory refers to the "underlying pattern in which the content is arranged, and is assumed to reflect aspects of autobiographical memory organization" (p. 601). Some theorists (e.g., Conway \& Holmes, 2004; Garro, 2001; Higgins, 1996; Woike et al., 1999) claim that motives, goals and desires drive autobiographical memory and thus, shape the content and structure of autobiographical memory (Conway \& Holmes, 2004; Woike et al., 1999). This allows autobiographical memory to be functional in that it aids an individual in clarifying their goals, discovering their identity, and deepening 
their understanding of significant life events and emotional personal experiences (Wang \& Conway, 2004). In this approach "autobiographical memory is not only a record it is a resource" (Robinson, 1986, p. 23) with autobiographical memory functions conceptually mapped into three broad purposes; a social function, a directive function and a self function (e.g., Bluck et al., 2005; Pillemer, 1992; Wang \& Conway, 2004).

\subsubsection{Social Function}

Bluck et al. (2005) state autobiographical memory is important in developing, maintaining and nurturing social bonds. Cohen (1998) claims the most basic social role of autobiographical memory is to provide material for conversation allowing for the enrichment of social interaction. Autobiographical memory is also thought to assist with empathy and an understanding of others (Cohen, 1998), providing others with information about one's self (especially in initial interactions) (Bluck et al., 2005) and in memory sharing, which strengthens the intimacy and social bonds of existing relationships (Bluck et al., 2005). Robinson and Swanson (1990) discuss the impact on social bonds when there is impairment in autobiographical remembering.

\subsubsection{Directive Function}

Bluck et al. (2005) claim that the directive role of autobiographical memory includes using the past to guide present thought, feeling and behavior. Cohen (1989, 1998) states autobiographical memory aids problem-solving and the development of opinions and attitudes, while Baddley (1987) argues that it aids in the comprehension of the past and prediction of future. Autobiographical memories are also believed to anchor personal values, act as originating events for chosen life directions and as turning points that redirect the life path (Bluck et al., 2005). They are also thought to update current views of the biographical self (Bluck et al., 2005) and allow for an understanding of the inner world of others, assisting in the prediction of others' behavior (Robinson \& Swanson, 1990).

\subsubsection{Self Function}

Of greatest relevance to this thesis is the self function of autobiographical memory. There is a theoretical connection between autobiographical memory and the concept of self. Early theorists (e.g. Greenwald, 1980; Kelly, 1955; Markus, 1977; 
Rogers, Kuiper, \& Kirker, 1977; Schachtel, 1947) prefigured this intrinsic association. For instance, James $(1890,1950)$ claimed that memory was a major component of the self. Kelly (1955) espoused that for personal experiences to be remembered clearly, they must be supported within an existing self-structure. More recently, theorists have developed this theoretical connection between the self and autobiographical memory (Wang, 2001). Conway and Holmes (1999) suggest that autobiographical memory defines the self. Conway and Tacchi (1996) even argue that autobiographical memories are intrinsically related so that autobiographical memory is a part of the self.

Recently, researchers (e.g., Conway \& Pleydell-Pearce, 2000; Wang \& Conway, 2004) have proposed that autobiographical memory assists in the definition of self, in the experience of personhood and in self-regulation. Conway (1990) believes autobiographical memory to be a key component and manifestation of selfknowledge and self-development. Autobiographical memory is also thought to preserve self-continuity and self-coherence over time and context (Barclay, 1996; Bluck, 2003; Bluck \& Levine, 1998; Brewer, 1986). Neisser (1988) states that knowledge of the self in the past and as predicted into the future is seen as an essential type of self-knowledge. Bluck et al. (2005) posit that autobiographical memory allows individuals to have and maintain a "biographical identity and to be able to maintain a coherent self-concept across an entire lifespan, even in the face of developmental change and life-events" (p. 110). Therefore, autobiographical memories are thought to be especially essential when the self is in adverse conditions that require self-change (Robinson, 1986).

Autobiographical memory is seen to be both fundamental and functional in the development and expression of a dynamic self-concept (Bruner, 1990; Fitzgerald, 1992; Fivush, 1994; Nelson, 1996; Pillemar, 1998; Singer \& Salavoy, 1993; Wang, 2001). At the same time these models suggest that the self operates on the encoding, organization, and retrieval of personally significant events (Brewer, 1986; Conway, 1996; Neisser, 1994; Ross \& Wilson, 2000; Wang, 2001). Specifically, Wang and Conway (2004) theorize that the self is instrumental in the encoding, organization and retrieval of autobiographical memory, while autobiographical memories function to develop, express and maintain the self. It is this intimate and reciprocal relationship with the self that has drawn autobiographical memory most clearly into the cultural sphere. This will be discussed at greater length in Chapter 3. 


\subsection{Processes other than Autobiographical Memory}

Alongside autobiographical memory other processes need to be included in PTSD models to provide a comprehensive model of etiology and maintenance of PTSD. Therefore, as will be apparent in the models outlined below, alongside autobiographical memories, PTSD models include processes such as goals, motivation, cognitive-affective reactions, appraisals, social support and cognitive coping strategies (Brewin \& Holmes, 2003).

\subsection{Socio-Cognitive Models of PTSD}

The chapter has highlighted how autobiographical memory came to be the focus of many of the current socio-cognitive theories of PTSD. Autobiographical memory has been defined and its functions outlined. Particular emphasis was dedicated to the self-function of autobiographical memory given autobiographical memory is thought to be especially essential when the self is in adverse conditions (such as trauma) (Robinson, 1986) and is understood to have an intimate and reciprocal relationship with the self. It is believed that this relationship connects autobiographical memory and culture and will be discussed more fully in the next chapter. This chapter will now proceed with an outline of a number of the most prominent of these socio-cognitive models of PTSD in order to highlight the current socio-cognitive models of PTSD, demonstrate the centrality of autobiographical memory in such models, and initiate an exploration of the cultural flexibility of such models. After the summary of each PTSD model, the psychological processes specific to the model that allow the model to be transported into the cultural realm, will be mentioned. A discussion of the impact of cultural differences in self on such concepts will be discussed in Chapter 3.

\subsubsection{Self Memory System (SMS)}

Conway's (Conway, 2005; Conway, Meares, \& Standart, 2004; Conway \& Pleydell-Pearce, 2000) self-memory system (SMS) is a cognitive model applied to a "wide range of autobiographical memory phenomena" (Conway \& Pleydell-Pearce, 2000 , p. 263), one of which is the phenomena of PTSD. It views autobiographical memory as a "resource of the self...used to sustain or change aspects of the self" (p. 
268). The SMS posits that autobiographical memory functions to validate and support self-schema, is critical to an individual's current goals and mental health, provides a stable self-system and contributes to specific aspects of the self such as self-beliefs.

The SMS is a superordinate system comprised of a working self operating in conjunction with a conceptual self and an autobiographical knowledge base. The working self is comprised of a motivational hierarchy of goals and sub-goals that operate to constrain cognition, and ultimately behavior, into effective ways of operating in the world (Conway \& Pleydell-Pearce, 2000). Simply, the working self is made up of a hierarchy of goals based on current individual motivations and aims (e.g., having a child, getting married, finding employment, completing a degree, etc.). Memory is regulated, encoded and integrated into the autobiographical knowledge base utilizing such motivational goals.

The autobiographical knowledge base is comprised of a hierarchy of knowledge: lifetime periods, general events and event-specific knowledge (ESK). Lifetime periods are the most general, abstract, inclusive type of knowledge and denote time periods typically measured in years. They have identifiable beginnings and endings, and contain thematic knowledge. An example of a lifetime period would be high school. General events are more specific, heterogeneous memories, typically measured in months, weeks and days. One prominent feature of general event clusters is that they "feature vivid memories of events relating to the attainment or failure to attain personal goals" (Conway \& Pleydell-Pearce, 2000, p. 264). Examples of general events include doing exams. ESK refers to memory for highly specific knowledge unique to a single event and typically measured in seconds, minutes, or possibly hours. An example of ESK is failing a year 8 English exam. ESK details are contextualized within a general event, which in turn is associated with one or more lifetime periods, which locate the more specific knowledge within an individual's autobiographical memory. The SMS proposes autobiographical memory is organized by themes (such as relationship theme, work theme, study theme, etc.) and these themes comprise one's life story.

Two retrieval routes are available to retrieve an autobiographical memory from the autobiographical knowledge base; the first is through high-order meaning based retrieval strategies (e.g., remembering one's wedding day), and, the second is through direct triggering by stimuli that were associated with the event (e.g., smells or sounds). The everyday memory is integrated into the autobiographical knowledge 
base and thus has connections to lifetime periods and other general events. This allows for elaboration of the memory, which enhances the first retrieval route and inhibits the second. Hence, when the memory enters awareness, it is compromised of specific information about the event, general information about the lifetime period that the event took place in, and abstracted information about the type of event in general. It is suggested that recall of ESK is taken as a defining feature of highly vivid memories and vivid memories are experiences in which self and goals are either highly integrated or strikingly disjunctive.

The SMS framework also recently recognized a working self-conceptual knowledge base, which alongside the working self regulates autobiographical remembering. Conway et al. (2004) refer to this as the conceptual self. The conceptual self recognizes and realizes "social constructed schema and categories that define the self, other people, and typical interactions with the surrounding world...drawn from the influences of familiar and peer socialization, schooling and religion, as well as the stories, fairy-tales, myths, and media influences that are constitutive of an individual's culture" (Conway, 2005, p. 597). The conceptual self consists of the life story alongside specified self-structures that exist independently of specific episodes such as personal scripts, self-with-other units relational schema, possible selves, selfguides, attitudes, values and beliefs (Conway, 2005).

The SMS considers autobiographical memories to be "primarily records of success or failure in goal attainment" (Conway \& Pleydell-Pearce, 2000, p. 266). Goals are embedded in the SMS with representation in the working self and archival connections in the knowledge bases. Autobiographical knowledge and conceptual self-knowledge constrains the goal structure of the working self and the working self determines what knowledge can be accessed and how that is to be constructed into memory. The working self, in order to maintain self-coherence between memories, goals, conceptual knowledge and the conceptual self, "modulates the accessibility of autobiographical knowledge" (Conway, 2005, p. 600). It does this to "ground the self in goal relevant self-defining experiences" (p. 600). This constrains the self in terms of what the self has been in the past, what the self is at present and what the self can be in the future. If current goals and plans of the working self are in opposition to autobiographical knowledge, then there has been a breakdown in the normal function of the SMS and depending on severity, the system may enter a pathological state. The SMS assumes that it is very difficult to change goals because changing one goal 
structure would often require changing many other goals. Hence, there appears to be a principle of conservatism, which operates to avoid change (Conway et al., 2004). Thus, individuals tend to remember features of an event that align with the working self. Alternatively, individuals change, add or omit aspects to or from the memory rather than change one's goals. A major objective of the SMS is to preserve selfcoherence.

\section{SMS and trauma memories.}

Conway and Pleydell-Pearce argue that traumatic experiences present a "threat to current plans and goals to which the working self cannot adapt" (p. 281). Trauma is a contradiction to one's goal hierarchies (e.g., one tends to hold goals suggesting being in control, the world being safe, people being good, etc.). Trauma violates or contradicts active goals, which might otherwise have been used to integrate the experience with the knowledge base. Hence, there are no currently active goals that can be made use of to mediate integration of the trauma memory into the autobiographical knowledge base. Instead, the trauma memory remains an ESK representation of the event, and is not contextualized within the autobiographical knowledge base/life story. As a result the trauma memory becomes associated with the activated working self and its goals - the very goals that were to be used for the integration of the memory. Hence, each time any of these associated goals are activated, the probability of activating the trauma ESK increases (i.e., reliving/intrusion symptoms of PTSD). Therefore the trauma memory is poorly elaborated and inadequately integrated into its context in time and place with other autobiographical memories and the conceptual self.

Unlike the everyday memory, the unintegrated trauma memory cannot be retrieved using the first retrieval route as it lacks the connections to other autobiographical memories. Therefore, the trauma memory is activated via the second retrieval route. Stimuli associated with the event activate the memory, reducing the ability to control retrieval. This explains the problem with the difficulty in intentionally recalling the event, the here and now aspect of the recollection, the absence of contextual links and the easy triggering of the memory by particular cues (Ehlers \& Clark, 2000). This model suggests that the excessive availability of the ESK may be reduced if it can be linked to contextualizing autobiographical knowledge such as lifetime periods or general events. 
In order to integrate the trauma memory into the life story, self-concept and self-knowledge the SMS may operate to change the trauma memory as a solution to the incongruence between the trauma event and the conceptual self. Conway describes examples where the self-system's response to trauma is to "lower the accessibility of memories of the events" or "even distort (the) memories" (2005, p. 599) with the motivation for these changes to maintain a sense of self-coherence and hence, integrate the memory into the life story and self-knowledge. The model suggests, as well, that over time self-consistency may need to be maintained by alteration in the person's self-construct/conceptual self leading to the development of a self-identity centered on being a victim of trauma or emphasizing self-change since the event (Conway, 2005).

The SMS in its incorporation of a conceptualization of self, alongside its emphasis on motivational properties (the working self) and existing knowledge structures (autobiographical knowledge base) makes this framework sympathetic to cultural considerations. The recognition of a conceptual knowledge base (a self that is, in part, constitutive of an individual's culture), which alongside the working self regulates autobiographical remembering, places this model, and its understanding of self and of autobiographical memory, clearly into the cultural sphere.

\subsubsection{Appraisal Model}

Ehlers and Clark's (2000) model converges on the SMS in that it also suggests differences in the nature of the memory of the traumatic event and its links to other autobiographical memories in those with PTSD compared to those without PTSD. They suggest the trauma memory of those with PTSD lacks the awareness of remembering that accompanies everyday autobiographical memories (autonoetic awareness). Similar to the SMS, the appraisal model posits that autobiographical events are usually incorporated into an autobiographical knowledge base, organized by theme and personal time periods and two retrieval routes are available to retrieve an autobiographical memory from this autobiographical knowledge base. The appraisal model, like the SMS, claims that for those with PTSD, the trauma memory is poorly elaborated and inadequately integrated into its context in time, place and other autobiographical memories. It is thought that a lack of memory integration may be due to an inability to establish a self-referential perspective during the trauma event - that is, the event is unable to be situated into a subjective context, comprised 
of both the internal (mental) and external (environmental) events that temporally coincide with the trauma event (Wheeler, Stuss, \& Tulving, 1997). As outlined in the SMS, the unintegrated trauma memory cannot be retrieved using high-order meaning based retrieval strategies, as it lacks the connections to other autobiographical memories but is rather activated via the direct triggering by stimuli associated with the event.

The appraisal model also proposes strong associations (Stimulus-Stimulus and Stimulus-Response associations) for traumatic material in memory, increasing the ease with which trauma memories and/or emotional responses are triggered. Ehlers and Clark state that two aspects of associative learning maintain PTSD. First, this form of learning aids the survivor in predicting what will happen next, even if the survivor is unaware of this. It seems that the stimuli present just prior to the trauma event will become associated with trauma and hence, by default when encountered in the future will predict threat to self. Second, retrieval of material learnt as a result of associative learning can occur without intention and an awareness of the cues that trigger remembrance of such material. Survivors may not even be aware that their emotional reaction (such as fear, anger, helplessness, etc.) was triggered as a result of a trauma cue related stimuli or more generally, trauma memory retrieval. The appraisal model also predicts strong perceptual priming (a form of implicit memory) for stimuli present during the traumatic event. Trauma associated cues (often outside of awareness), which can directly trigger the trauma memory, are more readily attended to following the trauma. Furthermore, given perceptual priming is related to implicit memory and implicit memory is poor at discriminating between memory traces, triggers that vaguely resemble stimuli present at the trauma are capable of triggering re-experiencing symptoms.

Extending the SMS, the appraisal model suggests that PTSD is not only the result of a disturbance or dysregulation of autobiographical memory but also the result of individuals' processing trauma in a way that has excessive negative appraisals of the trauma and/or its sequelae. Due to these appraisals, individuals process the past threat (i.e., the trauma event) as an on-going threat (either an external threat to safety or an internal threat to the self and the future) and hence, appraise future situations as dangerous. Once this perception of current threat is activated, "it is accompanied by intrusions, re-experiencing symptoms, arousal and anxiety symptoms, and other emotional responses" (p. 320), which motivates behavioral and cognitive responses 
that aim to reduce this perceived threat. In the short term this is effective but in the long term has the consequence of stopping cognitive change and hence, maintains PTSD.

Ehlers and Clark have identified a wide range of such negative appraisals. Some of these appraisals are focused on the trauma experience ("The next disaster will strike") and signal an overgeneralization of threat ("Others can know that I am a trauma victim"). Other negative appraisals are centered round one's actions and lack of control strategies during the trauma experience ("I was weak", "I deserved for this to happen") and mental defeat/perceived loss of psychological autonomy ("I am incompetent, inferior, unworthy"). Other appraisals include a focus on trauma sequelae, alienation ("I will always be alone and no one understands"), and other's reactions ("Others think I cannot cope"). Negative appraisals can also center on symptom meaning, permanent change and the future ("I am permanently damaged", "It will never change."). Survivors continue to perceive current situations as threatening and dangerous as they may perceive themselves as inadequate and unable to cope in current situations and retrieve information and memories that confirm this inadequacy. Appraisals about others and interpersonal relationships may maintain PTSD because survivors have on-going negative appraisals of interactions with others, and thus, may withdraw from social interactions and are thus, less likely to receive social support and to correct negative beliefs about themselves and others (Ehlers, Maercker, \& Boos, 2000). Empirical support for the appraisal model has been derived from research (e.g., Ehlers, et al., 2000; Ehlers, Clark, et al., 1998) investigating four of the above cognitive appraisal styles, which have received considerable attention in recent years; mental defeat, control strategies, alienation and permanent change. Research has demonstrated trauma survivors with PTSD have significantly more negative appraisals than trauma survivors without PTSD.

In sum, the appraisal model suggests that PTSD is the result of individual's processing trauma in a way that has excessive negative appraisals of the trauma and/or its sequelae and a disturbance of autobiographical memory characterized by poor elaboration and contextualization, strong associative memory and perceptual priming. The appraisal model in its focus on autobiographical memory, existing knowledge structures and its incorporation of appraisals (i.e., the way one interprets and gives meaning to events) makes this framework also sympathetic to cultural considerations. Additionally, the appraisal model's fundamental premise, which 
suggests individuals with poorer psychological adjustment following trauma process the past threat as an on-going internal threat to the self and hence, appraise future situations as dangerous, highlights the association between the self and appraisals, and the impact of trauma on such an association.

\subsubsection{The Schematic, Propositional, Analogue and Associative}

\section{Representational Systems (SPAARS) Model}

Dalgleish (2004) suggests that the key to understanding psychopathology from a cognitive approach is to acknowledge that information is represented in the mind in different ways. He, like others (e.g., Horowitz, 1986, 1997; Janof-Bulman, 1992), argue that following trauma there are often pervasive changes in the survivor's views of the self and world - basic assumptions about the self, world and future. This has been referred to as transformation of meaning. Pre-trauma world conceptualizations are that the world is controllable, safe and predictable and the self is viewed as a reasonably protected. These conceptions can be "severely damaged as a result of trauma" (p. 229). The world becomes uncontrollable, unpredictable and meaningless. The self is now vulnerable. Dalgleish also stresses the emotional content linked to PTSD. Fear appears to be the dominant emotion in PTSD, but sufferers often experience a range of other strong secondary emotions such as anger (Foa, Riggs, Massie, \& Yarczower, 1995), guilt, shame, disgust and sadness (Andrews, Brewin, Rose, \& Kirk, 2000; Grey, Holmes, \& Brewin, 2001; Jaycox \& Foa, 1996; Reynolds \& Brewin, 1998). The presence and intensity of these secondary emotions appears to have an impact on recovery from PTSD.

The SPAARS model suggests there are four explicit levels of mental representation when approaching emotions. First, the schematic level is similar to the notion of schema in that they represent abstract, generic knowledge. Second, propositional representations represent referential meaning in verbal form. Third, the analogical representational system contains memories in the form of visual, olfactory, auditory images. Finally, the associative representational system is similar to fear networks in that they connect the above representations of information. It is suggested that schema and emotions organize, activate and inhibit information.

This framework offers two routes to the generation of emotions. First, is the appraisal driven route. Events and event interpretations are "appraised at the schematic level of meaning in relation to an individual's goals" (p. 248). For example, 
"fear is generated when there is an appraisal of threat because a schema is constructed in working memory, which represents the possible future interruption or noncompletion of a valued goal" (p. 248). The second proposed route is an automatic route via associative representations. Emotions, activated without appraisal, are activated rather as a result of "biologically prepared, repeated or overlearned relationships" (p. 249). When emotions are activated they act as an "organizing force" within the SPAARS model (p. 249).

Like the SMS, within this model a traumatic event would cause the production of intense appraisal-driven fear, helplessness, and horror because goals such as that of personal survival are threatened. Information about the trauma, the experience of the event and the response to the event will be encoded in parallel as propositional, analogical, and schematic representations. They will be linked by associative representations. Hence, trauma memories are distributed across different representational systems rather than being stored in one place. After trauma, trauma related information across the different representations within SPAARS is appraised as incompatible and signals a threat to the supraordinate configuration of the schematic models of the world and of the self. This repeated appraisal of the incompatibility and threatening nature of the trauma-related representations in memory, results in PTSD symptomology as (a) there is always low-level activation of the fear mode and hence, a continuous state of being in danger (hyperarousal symptoms), (b) information related to trauma intrudes, by virtue of this activated state, into consciousness (flashbacks, nightmare, etc.), and (c) any cues will be selectively processed and may activate the trauma memory increasing the likelihood of re-experiencing symptoms. As PTSD symptoms persist, further associative connections linking the trauma and fear develop, are strengthened and hence, traumarelated cues are automatically able to activate the trauma memory.

The SPAARS model tends to adopt a structural approach to memory. However, its emphasis on transformation of meaning (i.e., changes in views of self, others, world and future following trauma), the explicit levels of mental representation at the schematic and propositional level, and emotion draws the SPAARS model into the realm of culture. 


\subsubsection{Trauma as Central to Identity Model}

Berntsen and Rubin $(2006,2007)$ also posit that trauma violates schema-driven expectations. They espouse that deviations from schema do not necessarily result in poor or fragmented memories. Their framework is based on research (e.g., Brewer \& Treyens, 1981; Brown \& Kuliuk, 1977; Rubin \& Kozin, 1984) demonstrating that information that deviates from schema enhances memory for that information. Berntsen and Rubin claim that the trauma memory, as a result of its distinctiveness and emotional charge, remains highly accessible and a cognitive reference point for the organization of other autobiographical memories, which continues to impact on the interpretation of non-traumatic experiences and future expectations. The trauma event becomes perceived overtime as "a major causal agent" (in the life story or autobiographical self) and "thus a highly salient turning point in the person's life" (Berntsen \& Rubin, 2006, p. 221). Typically, turning points are culturally expected transitional events that provide self-definition or change in self-definition through role change (e.g., choice of career, marriage, birth of first child). In Berntsen and Rubin's account, the perception of the trauma event as a turning point together with the requirement for an "internal consistency of the life story" (2006, p. 221) results in the role of being a trauma victim, or trauma survivor, becoming a reference point for everyday inferences and the salient and important component of identity. Their account points to maintenance of trauma centered identity through "culturally sanctioned role transitions" and "personal identification with the social roles that are culturally expected" (Berntsen \& Rubin, 2007, p. 420). The centrality of the trauma memory to personal identity and autobiographical memory results in Ehlers and Clark's (2000) notion of perceiving current situations as current threat.

\subsubsection{Multiple Self-Representations}

Brewin (Brewin \& Holmes, 2003), as part of his dual representation theory, outlines that in memory people are believed to have multiple self-representations or identities that compete to be retrieved. These identities provide a "series of high-level frameworks that summarize experiences with the world and with close relationships, and within which specific thoughts, images, or impulses are organized" (p. 359). In trauma survivors with PTSD there are common negative self identities provoked by the trauma experience such as "the self as powerless, the self as inferior, the self as nonexistent, the self as futureless" (p. 359). These cognitions are thought to develop 
as a result of the trauma experience making positive self-identities difficult to retrieve or negative identities that have resulted from previous traumas more readily retrieved. Hence there is retrieval competition between negative and positive self-identities. Brewin (Brewin \& Holmes, 2003) posits that these negative self-cognitions can be modified be exploring the existence of alternative identities that have been experienced by the PTSD sufferer and "tracing the links between these identities and the experience of trauma" (p. 359). This theory offers plausible explanations as to why trauma and its treatment sometimes has such a major impact on self-views, and thus, leaves the trauma survivor feeling changed in fundamental ways (Brewin \& Holmes, 2003).

Both Berntsen and Rubin's and Brewin's approaches are clearly drawn into the cultural sphere by their recognition and inclusion of identity, social roles, role transitions, life stories, life scripts and cultural expectations and sanctifications in both the development and maintenance of PTSD. Furthermore, both approaches clearly implicate the self.

\subsection{Summary of PTSD Models and their Current Links to the Self}

Since the introduction of PTSD into the DSM-III (1980) there have been extensive and impressive findings associated with the etiology, maintenance and treatment of PTSD. Remarkably, this "growth in knowledge has been accompanied by the development of increasingly sophisticated theories that have... [kept] pace with [these] new findings while at the same time remaining anchored in basic psychological research" (Brewin \& Holmes, 2003, p. 340). These models appear internally sound and account for much of the phenomena observed in PTSD. However, it appears that these impressive advances in theoretical development have now reached a point in which it is both appropriate and necessary to consider current understandings of the self, which extend to include cultural variation in self-construal. Many of the PTSD model's fundamental concepts have visible connections to the self allowing them to be linked to theories pertaining to the self and consequently making the models potentially viable to the consideration of cultural issues. However, at present the models appear to have stopped short of considering the theory of selfconstrual (discussed in Chapter 3) and its cultural implications. 
It is the purpose of this thesis to transport these theories via their existing links with the self into the domain of cultural theory and research. Specifically, the thesis will use the SMS' (Conway, 2005) incorporation of a conceptualization of self and its understanding of autobiographical memory to transport the SMS into the cultural realm. The appraisal model (Ehlers \& Clark, 2000) will be transported into the cultural sphere using its understanding of self, autobiographical memory, appraisals, and its premise that PTSD sufferers process past threat as an on-going internal threat to the self. The thesis will use the notion of transformation of meaning (Dalgleish, 2004; Horowitz, 1986, 1997; Janof-Bulman, 1992) to increase cultural understandings of the impact of trauma on prior beliefs about the self, world and others. Finally, the centrality of trauma approach (Berntsen \& Rubin, 2007) will be drawn into the cultural domain utilizing its conceptualization of autobiographical self, identity, selfdefinition and culturally sanctioned role transitions. Cultural consideration of these models will most commonly be achieved by utilizing the pathway of autobiographical memory given its unique relationship with the self and its centrality in many of the above models.

\subsection{Clinical Implications}

The socio-cultural models of PTSD have guided current clinical practice. Generally, the models (e.g., Conway, 2005; Ehlers \& Clark, 2000) predict that positive adaptation to trauma should involve the development of conceptual associations not only between separate memories of the event but also to existing autobiographical memories and current self-knowledge. Most contemporary clinical theories (e.g., Dalgleish, 2004) propose that elaboration of the trauma memories (using imaginal exposure and cognitive behavior therapy) is critical for positive outcome when treating people with disturbed adjustment following trauma. This often involves helping the person making sense of the trauma in respect to aspects of their self-image and goals and facilitating the integration of the trauma memory into existing self-knowledge (Hembree \& Foa, 2004). Additionally, components of cognitive therapy, such as self-schema work, aim to address trauma caused "vulnerable identities" such as "the Self as powerless...inferior...nonexistent ...futureless" (Brewin, 2003, p. 73) and works to integrate a client's current views of 
the self (e.g., I am a victim, I am damaged, I have no control) with pre-trauma views and the life story.

Furthermore, Herman (1992), influential in the development of treatment for trauma, suggests traumatic events overwhelm the ordinary systems of care, security and identity that give people a sense of control, connection and meaning. She claims that the two principal consequences of trauma are disempowerment (a loss of autonomy) and disconnection from others (a loss of relatedness/interdependence). She proposes that given these two consequences, treatment needs to focus on empowering the survivor and assisting the survivor in establishing new relationships. Herman claims that the "first principle of recovery is the empowerment of the survivor" and that the efforts of "others may offer advice, support, assistance, affection, and care, but not cure" (p. 133). The role of others is to help the survivor in "rebuilding a positive view of the self' (Herman, 1992, p. 63). Ehlers et al. (2000) also suggest the role of others is to assist the survivor in correcting negative beliefs about themselves and others. Herman believes that it is not until this regaining of autonomy has been achieved that a focus on "deepening relationships" with peers, lovers and family should be the focus of treatment (p. 205). Hence, there appears to be a clinical emphasis on increasing autonomy in trauma survivors and a secondary focus on the role of relatedness and interpersonal relationships in the treatment of maladaptive trauma responses.

\subsection{Chapter Summary}

Since the introduction of PTSD into the DSM-III (1980), there have been impressive advancements in the development of sophisticated theoretical models explaining much of the etiology and maintenance of PTSD. These models have guided much of current clinical practice. Given the personal significance of trauma and the autobiographical memory's role in maintaining the self in the face of adverse life events (Bluck et al., 2005), autobiographical memory is central to many of these models attempts to distinguish between those with and without disrupted psychological adjustment. Although the models differ in the way in which they conceptualize autobiographical memory and in their understanding of how trauma impacts on autobiographical memory, there is an agreement in proposing that autobiographical memory in those with PTSD shows particular types of disruption 
and dysregulation. Alongside autobiographical memory other processes have been included in these models to provide comprehensive explanations for the etiology and maintenance of PTSD. Such processes include motivation, pre and post trauma views (of world and self), schema, identity, self-concept, goals, and emotions.

However, it appears that these impressive advances in theoretical development have now reached a point in which it is both appropriate and necessary to consider current understandings of the self, which extend to include cultural variation in selfconstrual. Underpinning many of the concepts fundamental to the PTSD models is the notion of self. Nonetheless, the majority of these models have not taken up the challenge of fitting their propositions about autobiographical memory and other processes posited to be involved in the development and maintenance of PTSD with psychological understandings of the nature of self, and as a result they have not considered the impact of culture. It is the purpose of this thesis to transport these theories, via their existing links with the self, into the cultural realm. Particular focus will be given to autobiographical memory.

In order to move the PTSD models into the realm of cultural theory and research, the next chapter of this thesis outlines the theory of self-construal and consequently discusses cultural variation in self, the impact of cultural variation in self on autobiographical memory and the other processes implicated in the PTSD models. This will enable the final introductory chapter to establish a conceptual framework outlining the impact of cultural variation in self on the trauma autobiographical memory and subsequent implications for psychological adjustment. 


\section{CHAPTER 3}

\section{Culture, the Self, and Autobiographical Memory}

A number of theorists (e.g., Hofstede, 1980; Markus \& Kitayama, 1991; Sato, 2001) have suggested that people in different cultures have strikingly different understandings of the self. Such culturally diverging self-construals have been found to impact on, and in many circumstances govern, the very nature of individual experience, including behavior, cognition, goals, autobiographical memory, schema, appraisals, emotion, and motivation (Markus \& Kitayama, 1991). In other words, diverging self-construals has been found to impact on, and in many circumstances govern, the very fundamental elements to socio-cognitive models of PTSD. This chapter outlines prominent theories of cultural variation in self-construal and their impact on the concepts fundamental to current socio-cognitive models of PTSD. Particular attention will be given to autobiographical memory given its central role in PTSD models. The links between these concepts and cultural differences in self will be drawn upon to transport the PTSD models into the cultural sphere. Finally, general issues related to culture and PTSD will be discussed.

\subsection{Hofstede's Model of Cultural Dimensions}

Hofstede (e.g., 1980; Hofstede \& Hofstede, 2004), one of the founders of cross-cultural psychology, developed a model of cultural dimensions that accounts for cultural differences between societies. One such cultural dimension was the individualism-collectivism dimension - the degree of individual or group orientation within a society. Based on extensive research, Hofstede (1980; Hofstede \& Hofstede, 2004) ranked societies on the individualism-collectivism dimension. A high individualism ranking indicated that individuality, independence and autonomy are dominant within the society. A low individualism ranking indicated the society is more collectivistic and emphasizes close ties between individuals and relatedness (Hofstede \& Hofstede, 2004). Since the development of Hofstede's model, much research has advanced our understanding of this dimension. In fact, as Green, Deschamps, and Paez, (2005) state the individualism-collectivism construct is without a doubt, the most theoretically and empirically prevalent concept in cross-cultural psychology. 
Alongside this rapid expanse of literature, there has been developments (e.g., Bellah, Madsen, Sullivan, Swidler, \& Tipton, 1985; Hofstede, 1980, 2004; Markus \& Kitayama, 1991; Oyserman, Coon, \& Kemmelmeier, 2002; Triandis, 1995) in the operational definition of individualism and collectivism. Across these definitions it is suggested that in individualistic societies a person is considered to need a strong sense of autonomy. Typical attributes believed to be associated with individualism are independence, personal agency, self-reliance, uniqueness, achievement orientation, and competition (Green et al., 2005). Individualistic societies tend to be Western societies (e.g., Australia, Western Europe and North America) (Green et al., 2005; Hofstede \& Hofstede, 2004). In contrast, it is theorized that in collectivistic societies, the functioning unit is more often the group than the individual. Collectivism is thought to be associated with a sense of duty toward one's group, interdependence with others, a desire for social harmony, conformity to social norms, and roles and status are defined within collectives (Green et al., 2005; Sato, 2001). Collectivistic societies tend to be non-Western societies (e.g., Asia, South America, Africa and Eastern Europe) (Green et al., 2005; Hofstede \& Hofstede, 2004).

\subsection{Markus and Kitayama's Self-Construal Theory}

Unlike, Hofstede's model, which is directed at explaining cultural differences at the societal level, Markus and Kitayama $(1991,1994)$ developed the theory of selfconstrual, which pertains to cultural differences in the self. They posit that different cultures hold remarkably divergent construals of the self, others and the interdependence between the two. They state that in independent/individualistic cultures, the self is conceived as a unique, independent, self-contained, autonomous individual comprised of a particular arrangement of internal attributes (e.g., traits, abilities, motives, and values). The self behaves in a way that is consistent with these internal attributes and hence, is seen as detached from the social context. In interdependent/collectivistic cultures, the self is perceived as an interdependent entity attending to and fitting in with others. The self is perceived as interdependent with the surrounding context (social) and it is the other, or "self-in-relation-to-other", that is focus in the person's experience. Experiencing interdependence requires perceiving oneself as part of an "encompassing social relationship and recognizing that one's behavior is determined, contingent on, and, to a large extent organized by what the 
actor perceives to be the thoughts, feelings, and actions of others in the relationship" (1991, p. 226). Components of the self are hence seen to be dependent on the public components of the self or specific social roles. The interdependent self also has a collection of internal attributes but these internal attributes are considered to be situation specific.

Markus and Kitayama espouse that the key differences between an independent and interdependent self-construal are as follows. The structure of the independent self is bounded, unitary and stable, while the interdependent self is flexible and variable. Important features of the independent self-construal are private internal aspects of self (such as abilities, thoughts and emotion), whereas in interdependent self-construal external, public aspects of the self (such as social roles, relationships and status) are important. The tasks of the independent self are to be unique, express the self, realize internal attributes and promote own goals, while the tasks of the interdependent self are to belong, fit-in, occupy one's proper place, engage in appropriate action and promote other's goals. The role of others for the independent self-construal is self-evaluation (i.e., others are important for social comparison), whereas in interdependent self-construal others are important for selfdefinition (i.e., relationships with others in specific contexts define the self). Responsiveness to the social environment for the independent self-construal may be the result of the "need to strategically determine the best way to express or assert the internal attributes of the self. Others, or the social situation in general, are important, but primarily as standards of reflected appraisal, or as sources that can verify and affirm the inner core of the self" (1991, p. 224), whereas the interdependent selfconstrual may express and experience emotions, and adhere to goals and motivations that are significantly shaped and dominated by the reaction of others. Relationships for the independent self-construal may be viewed as a means for obtaining individual goals, while in interdependent self-construal relationships will often be ends in and of themselves.

Markus and Kitayama propose that such representations of self are most elaborated in memory. They suggest that these memory representations can be called (and have been called, e.g., Gergen, 1968; Markus, 1977; Stryker, 1986) core conceptions, salient identities, or self-schema. Such representations govern behavior enabling it to be culturally appropriate. Markus and Kitayama state whenever a task, event, or situation is self-relevant cognition, emotion and behavior are thought to be 
influenced by the nature of the self-schema. Cultural differences in self-construal have been found to be active in the regulation of processes related to self and other schema such as self-relevant information processing, motivation, and affect regulation, and in the management of relatedness processes such as social comparison, person perception, and the forming and development of social interactions (see Markus and Kitayama, 1991, for review).

The self-construal theory claims that the interdependent self-construal seems a relatively idealistic side of self when compared to the somewhat selfish independent self. However, Markus and Kitayama counter this notion by claiming that those with interdependent self-construal only act with responsive and cooperative actions when there is mutual support and group commitment. That is, interdependence is not shown non-discriminately to others but rather to "in-group" members such as family, social groups, and so forth.

Finally, variation exists to which individuals exhibit an independent versus interdependent orientation in self-construal both within and between collectivistic and individualistic cultures (Lee \& Zane, 1998; Miller \& Sperry, 1987, Nakamura, 1964; Spiro, 1993; Wang \& Hseuh, 2000). However, normative differences between collectivistic and individualistic cultures are marked (Fiske, Kitayama, Markus, \& Nisbett, 1998, Hsu, 1953; Kagitcibasi, 1996; Markus \& Kitayama, 1991; Triandis, 1989).

\subsection{Sato's Systems of Self-Organization Theory}

Sato's (2001) more recent theory of self-organization holds many similarities to the self-construal theory. He, like Markus and Kitayama, groups various components of self into two very similar clusters, autonomy/independence and relatedness/interdependence and asserts cultural differences in self-construal. However, Sato gives greater prominence to the notion that individuals are motivated to achieve both a sense of autonomy and relatedness, as both aspects are required for well-being, mental health and self-worth (Helgeson, 1994; Sato, 2001). Other theorists (e.g., Bonanno \& Singer, 1990; Maslow, 1970; McAdams, 1985; Sullivan, 1953) support Sato's assertion in that they posit that after physiological needs such as food, water and shelter, the need for autonomy and relatedness may be the two most fundamental human requirements. Sato claims that the two self-systems organize the 
self in relation to the environment so the person is constantly able to satisfy the requirement for both autonomy and relatedness. Hence, the two self-systems are continuously changing to meet the constantly changing requirements of the current circumstance. Furthermore, there is accumulating evidence (e.g., McGuire, McGuire, Chile, \& Fujioka, 1978; Trafimow, Triandis, \& Goto, 1991; Wagar \& Cohen, 2003; Wang \& Ross, 2005) indicating that the cultural dominance in the autonomy/relatedness distinction can be overridden by temporary primes that call forth the different orientation of the self. For example, Wang and Ross (2005) conducted two experiments exploring culture and self-priming effects on autobiographical memory retrieval. In their experiments either the collective/interdependent or private/independent aspect of self was primed prior to memory retrieval. They found priming effects did emerge; memory content reflected the particular aspect of self being primed. Additionally, Trafimow et al. (1991) found priming a particular aspect of the self increased the retrieval of self-cognitions pertaining to the primed aspect of the self.

Sato suggests that despite all individuals holding both self-construals, autonomy appears to be the one facet of the self most emphasized in individualistic cultures, whilst the concept of relatedness seems to be the one facet of the self emphasized in collectivistic cultures. He states that people with independent and interdependent self-construal differ in what they need to maintain a positive view of the self and to achieve mental well-being. He claims that individuals holding a strong independent self-construal need to view themselves as achieving personal success, and it is only if levels of personal achievement or control over their environment diminishes does depression and/or anxiety seem to result. In contrast, he states that individuals holding an interdependent self-construal have a need to be accepted by others, and it is only when alienation or isolation occur that depression and/or anxiety may result.

\subsection{Suh's Theory of Self-Consistency}

Suh $(2000,2002)$ theorized that an internally coherent self-identity is an essential ingredient for mental health in individualistic societies but not in collectivistic societies. He claims that Western theories of psychology suggest that a person should integrate various components of the self, be consistent across situations 
and information inconsistent with this congruent self-view must go through repair work so the information aligns with self-views. As outlined in Chapter 2, this is central to many of the PTSD models. This coincides well with independent cultures' emphasis on autonomy, as the individual rather than the situation is the anchor of behavior, thoughts and feelings, and the decontextualized self uses internal sources to guide behavior and derive meaning and purpose. Suh suggests that this does not hold equally true in collectivistic cultures where the focus is the social context rather than on the person. Suh suggests that being rigidly self-consistent can be viewed as personal immaturity or arrogance in these cultures as collectivistic cultures value variation in the self when engaging with differing self-relevant groups (e.g., family, work, school, etc.). Conformity to these groups and the social context is paramount.

\subsection{Summary of Cultural Models of Self}

The above theories highlight that people in different cultures have strikingly different understandings of the self, of others, and of the relationship between the two. The general consensus across these theories is that in individualistic cultures an independent self-construal is emphasized, while in collectivistic cultures an interdependent self-construal is emphasized. The independent self-construal is defined as separate from the social context, it is a bounded, unitary and stable entity, promoting private aspects of self and individual goals, and aiming to be autonomous, self-reliant and unique (Markus \& Kitayama, 1991). In contrast, the interdependent self-construal is seen as connected with the social context, it is thought to be flexible and variable, promoting external, public aspects of the self, and aiming to belong, occupy one's proper place and engage in appropriate action (Markus \& Kitayama, 1991). These diverging construals have been found to impact on, and in many circumstances govern, the very nature of individual experience, including behavior, identity, self-coherence and self-consistency, cognition, goals, memory, schema, appraisals, emotion, and motivation (Markus \& Kitayama, 1991; Sato, 2001; Suh, 2000). Despite this prevailing cultural difference in self-construal, individuals do have both aspects of self (Sato, 2001) and there is accumulating evidence (e.g., Trafimow et al., 1991) indicating that the cultural dominance in the autonomy/relatedness distinction can be overridden by temporary primes that call forth the different orientation of the self. 


\subsection{Self-Construal, Trauma and Psychological Adjustment}

The link between trauma and the two aspects of the self is most clearly demonstrated in Herman's (1992) assertion that the two principal consequences of trauma which the person needs to negotiate are disempowerment (or a loss of autonomy) and disconnection from others (or a loss of relatedness/interdependence). Furthermore, as presented in the previous chapter, many of the PTSD models (e.g., Dalgleish, 2004; Ehlers \& Clark, 2000; Janoff-Bulman, 1992) discuss post-trauma changes in various beliefs, assumptions, schema and appraisals held by the survivor about the self (e.g., worthiness, agency, vulnerability) and about interactions between the self and others (e.g., intimacy, safety, trust). According to Kolts, Robinson, and Tracy (2004), PTSD sufferers tend to experience disruptions in two basic areas: the ways in which they assign meaning to themselves, and the ways in which they assign meaning to the world (including social interactions) around them. They found in their study that students who were excessively dependent upon interpersonal relationships and students who were exceptionally autonomous had higher levels of PTSD symptoms. This observation indicates a possible role for autonomy and relatedness in the development of PTSD. Finally, the PTSD theories have fundamental processes and assumptions related to the self, which by default implicate cultural variation in self. The chapter will now proceed by focusing on the impact of cultural variation in self on these processes and assumptions and their relevance in the etiology and maintenance of PTSD.

\subsection{Cultural Differences in Self-Construal Influence on Autobiographical}

\section{Memory}

As stated above, Markus and Kitayama propose that representations of selfconstrual are most elaborated in memory. Recent developments (e.g., Wang \& Conway, 2004) in the literature reveal that cultural differences in self-construal impact on autobiographical memory. As outlined in Chapter 2, autobiographical memory is thought to aid goal clarification, identity development, and life event understanding (Woike et al., 1999). Events are believed to be remembered in such a way that is consistent with prior knowledge, expectations, and current motivations and goals (Conway, 2005). Given differences in self-construal have been found to 
impact on shaping such elements; culture is seen to shape autobiographical memories (Garro, 2001). Consequently, autobiographical memory is functional at the individual level, as it assists in "sustaining current goals, self-theories, attitudes and beliefs", and at the cultural level as it helps one be "congruent with their culture's goals, values and belief systems" (Wang \& Conway, 2004, p. 912). Wang and Conway theorize a unique, complex, symbiotic relationship between the self and autobiographical memory, where the self is instrumental in the encoding, organization and retrieval of autobiographical memories, and autobiographical memories in turn function to develop, express and maintain the culturally sanctioned self. This is evidenced in the following body of literature.

\subsubsection{Social Cultural Development Theory of Autobiographical Memory}

In Nelson and Fivush's (2004) social cultural development theory, autobiographical memory is understood to emerge gradually across the preschool years in the context of developments in language, memory, and self, to serve functional goals. Hence, they propose that cultural variations characterize autobiographical memory during this early development and into adulthood.

Nelson and Fivush posit that language is an essential social cultural instrument vital in the development of autobiographical memory. First, language is the way in which autobiographical memories are expressed, and hence, language is "instrumental in providing the organizational and evaluative forms characteristic of autobiographical memory" (p. 294). Second, children start discussing past experiences with others allowing them to become better at "forming organized representations of past experiences" (p. 294). Third, such practices assist children in realizing that memories are representations of past experiences and hence, others can evaluate the same event from different subjective perspectives. Based on Ross, Xun, and Wilson's (2002) work, it is suggested that cultural self-construal and associated beliefs may be learned through one's language. However, a thorough examination of this suggestion is beyond the scope of this thesis.

A second mechanism Nelson and Fivush claim to be essential in the development of autobiographical memory is the development of a cognitive sense of self or theory of mind (e.g., Howe \& Courage, 1993, 1997; Wang, 2001). However, Garro (2001) argues that a cultural theory of mind is also needed as it provides prior knowledge, expectations and it establishes the functions for memory. Hence, Garro 
suggests that alongside a sense of self, a child needs an understanding of the cultural processes, cultural cognitive processes, and a socially discrete and situated nature of cognition. Furthermore, it is posited that the sense of self and autobiographical memory emerge somewhat simultaneously allowing for the development of Wang and Conway's proposed unique relationship between the self and autobiographical memory, and enabling the social, self and directive functions of autobiographical memory to be achieved.

Nelson and Fivush understand parental reminiscing style to be another fundamental social cultural tool essential to the development of autobiographical memory. Theorists (e.g., Han, Leichtman, \& Wang, 1998; Wang \& Fivush, 2005) suggest that the manner in which autobiographical memory is encoded and retrieved in childhood, is a consequence of co-construction between the developing child and significant others. That is, as language develops, the child begins to discuss past events with their parents allowing for the child to learn the dominant narrative structure; specifically, what is socially and culturally appropriate and valued (Han et al., 1998). Family reminiscing is believed to help establish social connectiveness and individual uniqueness in the child and thus, creates both social (relatedness) and personal (autonomous) identities (Wang \& Fivush, 2005).

Han et al. (1998) proposes that parental reminiscing styles differ across cultures as "local habits relevant to personal remembering about the past, general rules of social interaction, the nature of social hierarchy and parents' beliefs about the role of children" (p. 702) all influence how the past is discussed within a culture. One such cultural impact is the independent-interdependent distinction; "having an interdependent self as opposed to an independent self concerns the ways in which knowledge about self and other is processed, organized, and retrieved from memory" (Markus \& Kitayama, 1991, p. 232). Nelson and Fivush state that for autobiographical memory, individualistic cultures foster a more detailed sense of self in the past, while collectivistic cultures downplay the independent self in favor of the communal self.

Research has shown support for cultural differences in parental reminiscing style (Han et al., 1998). Mullen and Yi (1995) conducted a study with Korean and American mothers and their 40-month-old children. They found that the American dyads engaged in past talk three times as often as Korean dyads. American mothers also tended to be more detailed than Korean mothers when discussing all aspects of past events except social norms. Choi (1992) did a similar study using Canadian and 
Korean mothers. In this study it was demonstrated that Canadian mothers encouraged their children to contribute their ideas to the discussion, followed up and elaborated on their children's utterances, and took a partnership role rather than a leadership role in the conversation. While, Korean mothers tended to prompt their children to confirm the information they had already presented to them, tended not to encourage their children to introduce their own ideas into the discussion, produced more utterances unrelated to their children's previous utterances and expected the children to follow their lead. Wang and Fivush (2005) using American and Chinese mothers and their 3year old children, established that American mothers were more interactive, and used more elaborative conversations that focused on the child's role and predilections. Chinese mothers took a more directive role in posing and repeating memory questions and focused on social interactions.

In sum, the above findings indicate that Asian (collectivistic culture) mothers engage their children in reminiscing that encourages on-going relationships and model to the children to construct personal stories that give way to social interactions and collectivity (Wang \& Fivush, 2005). Nelson and Fivush (2004) posit that the ability to create a shared past with a focus on the goals of family and community allows individuals to enter into communities or cultures. In contrast, Wang and Fivush (2005) suggest mothers from individualistic cultures invite children to actively participate in the creation of their own life story, and Nelson and Fivush state that in these cultures more depends on the individual's self-definition, the self story and self as an active mediator in the creation of one's life direction. Quoting Nelson and Fivush, the way in which the "past is understood, constructed, and differentiated will be very much a function of the kinds of cultural values and goals that are placed on the shared understanding of a past" providing "children with information about how to be a self in their culture" (p. 506).

There is also research supporting cultural differences in aspects of autobiographical memories. Research (e.g. Pillemer, 1998) has indicated that selfrevealing, free-flowing, lengthier accounts of specific, one-point-in-time personal events are more prevalent among individualistic cultures than collectivistic cultures. Wang, Leichtman, and Davies (2000) demonstrated that American children tend to provide more elaborate, specific and self-focused autobiographical memories than their Asian peers. Wang (2001) found that American students reported lengthy, specific, self-focused, and emotionally elaborate childhood autobiographical 
memories. In contrast, Chinese students retrieved shorter childhood autobiographical memories focusing on collective activities, general routines, and emotionally neutral events. Han et al. (1998) showed that American children used more references to specific past events, descriptives, and references to internal states such as evaluations and had more mention of themselves than of others, than Asian children.

These cultural differences carry over into adulthood. For example, Wang and Conway (2004) asked American and Chinese participants to remember twenty memories from any period in their life. American adults recalled individual experiences, unique one-time events, and focused on their own roles and emotions. Chinese participants recalled social and historical events that emphasized social interactions and significant others. As Wang (2001) claims, in individualistic cultures a coherent, elaborate, well-integrated life story with the individual cast as the "lead" is vital as it provides self-understanding, self-continuity and affirmation of the self as an autonomous unit, whereas in collectivistic cultures, collective activities are often esteemed over this unique life story.

Trafimow et al. (1991) suggest cultural differences in independentinterdependent orientation may impact on cognitive organization. They proposed two competing theories concerning the organization of self-cognitions. First, both private/independent and collective/interdependent "cognitions may be stored in a single cognitive structure (i.e., the same place in memory), but with no internal organization" (p. 649). Hence, if this alternative is supported, the likelihood of retrieving a particular type of self-cognition (e.g., a collective self-cognition) would be determined by the frequency with which that type of self-cognition is represented, relative to the total number of self-cognitions. The second possibility was that selfcognitions are organized according to whether they pertain to the private/or collective self. That is, independent self-cognitions are organized around a general independent self-concept, while interdependent self-cognitions are organized around a general interdependent self-concept. They conducted two experiments to test which of the two alternatives was supported. The findings from both experiments supported the second proposal; private and collective self-cognitions are stored in separate locations in memory. Their findings suggest that self-construal may not only impact on the contents and structure of autobiographical memory but also on the organization of autobiographical memory. 


\subsection{Linking Theoretical Stances and Empirical Findings of Cultural Variations in Autobiographical Memory with PTSD Models}

These findings suggest that the content, quality and mechanisms of autobiographical memory are culturally distinct and instrumental in reaffirming the culturally sanctioned self. However the question remains, what does this mean for the autobiographical memory of trauma? Do autobiographical memories of trauma also reflect the cultural differences in the content and structure evident in everyday autobiographical memories? What is the role of trauma memories in the reaffirmation of the self? The thesis will now link theoretical and empirical advances concerning autobiographical memory and culture, with the PTSD models' accounts (as outlined in Chapter 2) of the role of autobiographical memory in the development and maintenance of PTSD.

First, some of the socio-cognitive models (e.g., appraisal model, Ehlers \& Clark, 2000; SMS, Conway, 2005; SPAARS, Dalgleish, 2004) posit that the trauma autobiographical memory is a contradiction to current goal hierarchies and plans of the autobiographical memory system. These models tend to suggest that trauma threatens goals of survival, agency and personal control. However, such goals of agency and personal control seem better aligned with the independent self rather than the interdependent self. According to Wang (2001), while for individualistic cultures the contents and structure of autobiographical memories operate to allow the individual to be autonomous and assert personal control in an event, in collectivistic cultures the content and structure of autobiographical memories esteems collective events and relatedness over personal agency. Hence, does trauma challenge goals of the autobiographical memory system in culturally similar ways? It seems possible that trauma may threaten goals in a universal way given evolutionary instincts to survive. Alternatively, it is conceivable that trauma may impact on autonomous goals in individualistic cultures and relatedness goals in collectivistic cultures.

Second, socio-cognitive models of PTSD (e.g., appraisal model, Ehlers \& Clark, 2000; SMS, Conway, 2005) suggest the trauma memory cannot be integrated into the autobiographical knowledge base but rather becomes associated with the activated working self and its goals. Research needs to consider if these activated goals, and the relationship between the trauma memory and these activated goals, differ culturally. Furthermore, the models suggest the trauma memory is inadequately 
elaborated and integrated into its context with "other autobiographical memories". Consideration is needed in terms of this integration, especially for clinical work, given "other autobiographical memories" reflect, both in content and structure, the culturally dominant self. Additionally, Ehlers and Clark (2000) believe that a lack of memory integration may be due to an inability to establish a self-referential perspective during the trauma event. However, research (e.g., Nelson \& Fivush, 2004; Wang, 2001) has repeatedly shown that while those from individualistic cultures encode autobiographical memories in a way that promotes a self-referential perspective, those from collectivistic cultures encode autobiographical memories in a way that promotes the collective and communal self. Berntsen and Rubin (2007) theorize that the trauma memory can become central to the organization of other autobiographical memories. While Wang and Conway (2004) posit that the self is instrumental in the organization of autobiographical memories, the impact of the centrality of the trauma memory on culturally differing autobiographical memory organizations remains unknown.

Third, some of the PTSD models (e.g., Berntsen \& Rubin, 2006, 2007; SMS, Conway, 2005) posit that following trauma there are changes in the trauma autobiographical memory to maintain and achieve self-consistency. However, Wang (2001) suggests that the contents and structure of autobiographical memory tends to only lead to self-continuity in individualistic cultures. Therefore, is there less distortion and alteration of the trauma memory in collectivistic cultures?

Fourth, models of PTSD (e.g., SPAARS, Dalgleish, 2004) focus on the role of emotion in the autobiographical remembering of the trauma memory. However, Wang (2001) has demonstrated cultural differences in emotional content in autobiographical remembering with individualistic cultures elaborating emotional content, while collectivistic cultures tend to downplay emotional content. Research needs to consider if this cultural difference in emotional content is also evident in the trauma memory. If cultural differences in emotion in the trauma memory do emerge, models may need to account for such cultural differences especially given some models (e.g., SPAARS) believe emotions to be an organizing force of the autobiographical memory system. However, it seems possible given the emotional charge and salience of the trauma event and the notion (e.g., Dalgleish, 2004; LeDoux, 1996) that the emotional responses of the trauma can become biologically prepared, repeated, or overlearned 
responses (suggesting an universal, biological emotional response to trauma), that the emotional content in the trauma memory may be somewhat culturally similar.

Finally, clinically, the models (e.g., appraisal model, Ehlers \& Clark, 2000; SMS, Conway, 2005) predict that positive adaptation to trauma should involve the development of conceptual associations between separate memories of the event and existing autobiographical memories. Most contemporary clinical theories (e.g., Dalgleish, 2004) propose that elaboration of the trauma memories is critical for positive outcome in treating people with disturbed adjustment following trauma. Hence, it seems essential that research be conducted regarding the trauma memory and its relationship to existing autobiographical memories which appear to differ culturally in terms of content and structure.

\subsection{Cultural Differences in Self-Construal Influence on other Processes Implicated in PTSD Models}

The cross-cultural theorists (e.g., Markus \& Kitayama, 1991) reason that diverging self-construals impact on, and in many circumstances govern, the very concepts believed to be instrumental in the development and maintenance of PTSD. The research supporting this proposition will now be reviewed and consequently the thesis will establish links that will be important in extending the cultural appropriateness of the PTSD models. It is worth noting, that the research exploring the impact of cultural differences in self on the various constructs is extensive and goes beyond the breadth of this thesis, thus, only the most relevant studies have been selected to illustrate cultural differences.

\subsubsection{Appraisals}

Mesquita and Walker (2003) demonstrate, both theoretically and empirically, that cultural differences in self-construal impact on the way in which events, situations and life encounters are appraised. They posit that cultural differences in self-construal "facilitate and render desirable certain appraisals of events, while making the occurrence of others less likely and less valued" (p. 784). Additionally, they suggest cultural variation in self-construal "foster culture-specific appraisal tendencies that are reflected in culturally distinct patterns of emotional experience" (p. 784). 
According to Markus and Kitayma's (1991) theory, individualistic cultures view success through independent, personal accomplishment and a personal sense of control, while in collectivistic cultures, Mesquita and Walker suggest, "agency is differently instantiated (e.g., by magic spells, ancestors, spirits) or is not valued as much" (p. 785) but rather fate, the multi-determination of events and the interdependence of an individual and their social environment are stressed (e.g., Fiske et al., 1998; Heine, Lehman, Markus, \& Kitayma, 1999; Nisbett, Peng, Choi, \& Norenzayan, 2001). Personal agency is believed to have "very limited applicability" in collectivistic cultures (Mesquita \& Walker, 2003, p. 785). Mesquita and Walker suggest that collectivistic cultures "emphasize secondary control or adjustment to the situation, as situations are not considered subject to personal influence" (p. 785).

There is abundant empirical support for their argument. Research (e.g., Mauro, Sato, \& Tucker, 1992; Mesquita \& Markus, 2004; Scherer, 1997) demonstrates that the tendency to attribute agency appears to differ consistently across cultures. Mauro et al. (1992), studying emotional appraisal in students from individualistic and collectivistic cultures has repeatedly found substantial cross-cultural differences in the dimensions of control, responsibility and agency. Mesquita and Ellsworth (2001) found that Americans tend to appraise positive and negative events in terms of autonomy, whereas agency/autonomy was much less prevalent in collectivistic cultures. Mesquita and Walker (2003) reviewed questionnaire studies in which participants were asked to remember an instance of a given emotion, describe the situation, and then rate the situation on a number of appraisal scales. These studies (e.g., Matsumoto, Kudoh, Scherer, \& Wallbott, 1988) have found that participants from individualistic cultures, when compared to participants from collectivistic cultures, are more likely to judge the dimension of responsibility to be applicable to emotional situations (Mesquita \& Walker, 2003).

Additionally, such cultural differences in appraisals have been found to impact on affective responses. Mesquita and Karasawa (2002) found in a study monitoring daily emotions of Japanese and American students that the impacts of agency appraisals to subjective experience differ culturally. For American students, personal responsibility and control predicted positive emotion, whereas for

Japanese students this was not the case. Mesquita and Walker (2003) summarized that there are cultural differences in agency appraisals, with individualistic cultures appraising emotional events as more relevant to the dimensions of responsibility, 
agency and control than collectivistic cultures. They continue with the suggestion that there is some evidence (Peng \& Nisbett, 1999) that "not feeling in control may be the default state of mind in many cultures [collectivistic], where the world tends to be perceived as one in which an individual has no control" (p.787). They suggest that it is most likely that some of the "detrimental effects of uncontrollability are specific to those cultures [individualistic] whose...[self-construal] contain assumptions of individuals having control, and where the uncontrollable is a violation of expectations and norms" (p. 787). Therefore, it may be that the violation of cultural expectations of personal control is deemed stressful, rather than the uncontrollability in and of itself being stressful.

\subsubsection{Identity}

Markus and Kitayama propose that in independent cultures identity is derived from a set of internal personal attributes, whereas in interdependent cultures identity is derived from others, and relationships with others, in specific contexts. This has been demonstrated in numerous studies (e.g., Cousins, 1989) using the Twenty Statements Test (TST; Kuhn \& McPartland, 1954). The TST asks people to respond to the question "Who am I?" by providing twenty responses following the phrase "I am". It is continuously demonstrated that those from independent cultures provide responses related to private inner aspects of self (e.g., I am friendly, I am kind, I am happy, etc.), while those from interdependent cultures provide responses associated with public aspects of the self or social roles (e.g., I am a mother, I am a friend, I am Chinese, etc.).

\subsubsection{Self-Coherence}

Suh $(2000,2002)$ theorized that self-coherence and self-consistency needs are culturally variable. Individualistic cultures value self-coherence and self-consistency as it aligns with independent culture's emphasis on autonomy. However, Suh suggests that in collectivistic cultures self-consistency is viewed as inappropriate and rather the focus is on the social context. Suh $(2000,2002)$ alongside others (e.g., Kanagawa, Cross, \& Markus, 2001) have found empirical support for this prediction. For example, Suh (2002) explored this by asking participants to rate how accurately twenty different personality traits described themselves in general and when interacting in five different social roles. It was found that the self views of Korean 
students were more flexible across social roles and contexts than that of Americans, suggesting self-consistency needs are weaker in collectivistic than individualistic cultures.

\subsubsection{Motivation and Goals}

Cultural variations in self appear to translate into cultural differences in motivation and goals. Based on empirical findings, Mesquita and Walker (2003) posit that those from individualistic cultures hold goals of standing out, being distinct from others and personally achieving, whereas those from collectivistic cultures aim to meet social obligations and responsibilities to maintain group harmony. Events in turn are created and promoted to allow goal obtainment. Lee, Aaker, and Garner (2000) found that individualistic cultures emphasize the approach of positive outcomes, whereas collectivistic cultures focus on the avoidance of bad outcomes. This is thought to align with cultural differences in self as individualistic cultures emphasize strengthening good qualities and in turn become autonomous and unique, whereas collectivists desire to live up to obligations and responsibilities and thus, the aim is to avoid bad outcomes (Mesquita \& Walker, 2003).

\subsubsection{Schema and Assumptions}

Markus and Kitayama (1991) hypothesize that the independent versus interdependent construals of self are among the most overarching schema of the individual's self-system. They suggest that these construals engage and organize the more specific self-regulatory schemata. Hence, they proceed to propose that the precise organization of many self-relevant processes and their outcomes relies essentially on whether these processes are rooted in an independent or interdependent construal of the self. Markus and Kitayama note that in the process of giving meaning and coherence to the world it is known that people show an increased sensitivity to self-relevant stimuli. For those with an independent self-construal, this includes information relevant to one's self-defining attributes but for those with an interdependent self-construal, such stimuli would include information about significant others within the person's social context or information about the self in relation to another person. Research (Eagle, 2005; Liem, Lim, \& Liem, 2000; Schwartz \& Kim, 2002) tends to support cultural differences in self impact on schema especially in self-schemas orienting person to self and others. Accordingly, Markus 
and Kitayama suggest that divergent self-construals have various consequences for all cognition relevant to self, others, or social relationships.

\subsubsection{Emotions}

Liem et al. (2000) claim that the "centrality of independence versus interdependence to one's sense of self is thought to influence one's affective response to life encounters" (p. 25). Many current emotion theorists (e.g., Ellsworth, 1994; Mesquita \& Walker, 2003; Scherer, 1984) conceive emotions to be the result of multiple aspects, such as appraisals, action readiness, autonomic nervous system activity, and behavioral goal setting. Mesquita and Walker (2003) theorize that cultural differences in self-construal can impact on differences in antecedent events, perceived significance of events, perceived focus of attention during an event and

differences in appraisals, which all impact on emotional outcome. Research (e.g., Liem et al., 2000; Markus \& Kitayama, 1994) has identified that other-focused emotions (emotions that have another as the primary referent e.g., shame, empathy) are more common in collectivistic cultures and ego-focused emotions (emotions centered on the individual and involve experiences such as self-affirmation, blocked autonomy or threat to one's integrity e.g., guilt, pride) are more prevalent in individualistic cultures. Stipek, Weiner, and Li's (1989) findings suggest that people with independent self-construal, when experiencing guilt, consider it caused by violations of laws or moral principles causing transgression of internal standards, whereas amongst Chinese, guilt is considered to arise when one hurts others, referring to the interpersonal relationship. Feldman Barrett and Russell (1999) found cultural differences in emotion valence. Kitayama, Markus, and Kurokawa (2000) found that Americans reported a much higher frequency of positive than negative emotions, whereas in the Japanese group there was no difference between frequencies of negative and positive emotions reported.

\subsection{Linking Theory and Empirical Findings with PTSD Models}

The cross-cultural theorists (e.g., Markus \& Kitayama, 1991; Mesquita \& Walker, 2003) reason that diverging self-construals impact on, and in many circumstances dominate, the very concepts believed to be critical in understanding the development and maintenance of PTSD. The purpose of this next section of the thesis 
is to generate issues and concerns that arise when theories of cultural variation in selfconstrual, and associated research, are applied to PTSD models. Hence, a number of questions arise, which will be addressed in the next chapter.

The appraisal model (Ehlers \& Clark, 2000) posits that appraisals differentiate between those with adaptive adjustment from those with maladaptive adjustment. Negative appraisals about the trauma, self, other, world and trauma sequelae tend to promote the development and maintenance of PTSD, as trauma survivors continue to perceive the threat as on-going and current situations as dangerous. Mesquita and Walker (2003) demonstrate, both theoretically and empirically, that cultural differences in self-construal impact on the way in which events, situations and life encounters are appraised; individualistic cultures appraise situations as being under personal control, while in collectivistic cultures situations are appraised less in terms of personal agency but rather the interdependence of an individual and their social environment is stressed. Additionally, such cultural differences in appraisals have been found to impact on affective responses; individualistic cultures tend to obtain more positive affect as the result of agency in a situation, while this tends not to be the case in collectivistic cultures. Thus, the question is posed; what impact do cultural differences in appraisals have on Ehlers and Clark's conceptualization of appraisals and the proposed role of appraisals in the development and maintenance of PTSD?

Berntsen and Rubin (2007), in their centrality of trauma approach, argue that the trauma memory can become central to personal identity. Markus and Kitayama propose that in independent culture identity is derived from a set of internal personal attributes, whereas in interdependent cultures identity is derived from others and relationships with others in specific contexts. Hence, consideration needs to be given to the influence of cultural variation in self-construal on the relationship between trauma and identity.

SMS (Conway, 2005) and the centrality of trauma approach (Berntsen \& Rubin, 2007) privilege self-consistency in their models. Suh $(2000,2002)$ theorized that self-coherence and self-consistency needs are culturally variable. Thus, do collectivistic cultures distort the trauma memory and alter self-concept in the same way as do individualistic cultures to achieve self-consistency?

Socio-cognitive models (e.g., appraisal model, Ehlers \& Clark, 2000; SMS, Conway, 2005; SPAARS, Dalgleish, 2004) suggest trauma threatens goals and motivation of the autobiographical memory system. Cultural variation in self has been 
found to moderate motivation and goals. Based on empirical findings, Mesquita and Walker (2003) posit that those from individualistic cultures hold goals of standing out, being distinct from others and personally achieving, whereas those from collectivistic cultures aim to meet social obligations and responsibilities to maintain group harmony. It is clear how such cultural differences in goals are utilized by autobiographical memory systems in the personal remembering of everyday events, it is not clear however, how such goals are used in the autobiographical remembering of trauma.

Dalgleish (2004) discusses the notion of schema of self, others and world, the impact of trauma on such schema and the role of emotions in differentiating between those with and without PTSD. Markus and Kitayama hypothesize that the independent versus interdependent construals of self are among the most overarching schema of the individual's self-system. They suggest that these construals engage and organize the more specific self-regulatory schemata. Accordingly, Markus and Kitayama suggest that divergent self-construals have various consequences for all cognition relevant to self, others, or social relationships. Liem et al., (2000) claim that the centrality of independence versus interdependence to the self is thought to influence one's affective response to life encounters. Thus, consideration is needed in terms of the impact of cultural differences in self on trauma's moderation of schema and emotions.

\subsection{Summary}

This section of Chapter 3 indicated how cultural theories of self have been found to impact on the concepts fundamental to current socio-cognitive models of PTSD. Specifically, it was demonstrated that diverging construals have been found to impact on, often to the extent of governing, the very nature of individual experience, including autobiographical memory, appraisals, identity, self-consistency, motivation and goals, schema and assumptions and emotion; elements deemed to be fundamental in the development and maintenance of PTSD. Cultural differences in self-construal impact on these elements and these elements in turn appear to function to develop, express and maintain the culturally sanctioned self. It is in this unique relationship

that draws our PTSD models most clearly in the cultural realm. Namely, it is uncertain whether cultural variations in self impact on these concepts in the event of 
trauma in a similar way to that found in general everyday events. If it is found that cultural variations in self-construal do impact on these concepts in response to trauma, the next question is, are our socio-cognitive models of PTSD flexible enough to encompass these cultural variations? Further discussion of such issues will remain until the next chapter. However, this present chapter will conclude with some final remarks pertaining to the topic of culture, PTSD and trauma.

\subsection{Culture and Clinical Psychology}

Besides these conceptual considerations, a more pragmatic reason for conducting this research is, despite the developments over the past two decades in research examining the relationship between culture and psychopathology (Draguns \& Tanaka-Matsumi, 2003), that there is still a gross imbalance in cross-cultural clinical research. Arrindell (2003) found that less than $1 \%$ of published articles in leading peer reviewed journals described psychological interventions in collectivistic cultures. Patel and Sumathipala (2001) concluded that this gross underrepresenttaion has negative implications for clinical psychology. This gross underrepresentation and limited understanding of cross-cultural clinical psychology extends to our current knowledge of PTSD. The socio-cognitive theories of PTSD have their underpinnings in individualistic theory and are based and supported on research conducted primarily in individualistic populations. Therefore, it remains unknown as to whether these cognitive theories are culturally bound or flexible enough to accommodate cultural variation in self.

This field of research has clinical implications for multicultural Western societies, for psychosocial work with populations at high risk of trauma exposure, such as refugees and indigenous populations, and for psychology in non-Western cultures, in areas such as assessment and intervention (Arrindell, 2003). At present, ethnic groups often do not receive the required assessment or psychological assistance (Arrindell, 2003; Dana, 2000; Draguns \& Tanaka-Matsumi, 2003), which Dana argues stems from a Eurocentric worldview and ethnocentrism that minimizes cultural variation, which in turn infiltrates into the assessment and treatment procedures. Finally, there is a general need to refine preventions, early interventions and treatments to make them more culturally appropriate. 


\subsection{Chapter Summary}

This chapter commenced with a summary of the prominent cultural theories of self-construal. Such theories posit that people in different cultures have strikingly different understandings of the self. The overarching consensus across these theories is that in individualistic cultures an independent self-construal is emphasized whilst in collectivistic cultures an interdependent self-construal is emphasized. The independent self-construal is defined as separate from the social context, promotes private aspects of self and aims to be autonomous, self-reliant, unique, express the self, realize internal attributes and promote own goals. In contrast, the interdependent self-construal is seen as connected with the social context, promotes external, public aspects of the self and aims to belong, occupy one's proper place, engage in appropriate action and promote other's goals. The chapter examined the impact of these diverging construals on the nature of individual experience, including autobiographical memory, appraisals, identity, self-coherence and self-consistency, motivation and goals, schema and emotions. Such concepts are posited by the sociocognitive models of PTSD to be involved in the etiology and maintenance of PTSD.

It is in the unique relationship between the self and the components (i.e., autobiographical memory, appraisals, goals, etc.) the PTSD models argue to be active in the etiology and maintenance of PTSD which draws our PTSD models most clearly in the cultural realm. The objective of Chapter 4 is to develop a conceptual framework achieving this amalgamation of the PTSD models with current understanding of the self. 


\section{CHAPTER 4 \\ Conceptual Framework}

\subsection{Review}

Chapter 2 provided an account of the prominent socio-cognitive models of PTSD. In the majority of these models the central focus was on the role of autobiographical memory in distinguishing between those with and without disrupted adjustment following trauma. Furthermore, these models also emphasized that other cognitive concepts such as appraisals, world-views, self-views and schema, and noncognitive concepts such as emotions, goals and motivation play a role in distinguishing between those who develop PTSD from those who do not.

Chapter 3 provided an outline of the prominent theories of cultural differences in self-construal. The chapter demonstrated that the cultural distinction in self has been repeatedly found to impact on autobiographical memory, appraisals, identity, self-consistency needs, motivation, goals, schema, assumptions, and emotions - the concepts the socio-cognitive models of PTSD posit are instrumental in the development and maintenance of PTSD.

\subsection{Conceptual Framework}

It seems both timely and necessary to expand the cultural understanding of these PTSD models using their existing theoretical connections with the self. Without this move, the models appear to be limited in that they have not considered the impact of the cultural distinction in self-construal. There has been very limited research to demonstrate whether the models are flexible enough to accommodate cultural variation in self or whether these models are culturally bound to an individualistic approach to selfhood. The objective of this chapter is to unite the two areas of research outlined in these previous chapters by developing a framework that encompasses both theory and research pertaining to the socio-cognitive models of PTSD and cultural differences in self-construal. Hypotheses pertaining to this thesis will be derived from this framework. 


\subsubsection{Background}

Of the various models relevant to understanding PTSD, Conway's (e.g., 2005) Self Memory System incorporates a conceptualization of self, making it potentially sympathetic to cultural considerations. It provides the initial framework for this thesis. Conway theorizes that SMS consists of three main components - the working self, the autobiographical knowledge base and the conceptual self. The construct of the conceptual self, as a socially and culturally derived self, places SMS, and its understanding of self and of autobiographical memory, clearly into the cultural sphere. According to Conway the conceptual self is socially and culturally constructed and is comprised of personal scripts, self-with-other units relational schema, possible selves, self-guides, attitudes, values and beliefs. Markus and Kitayama (1991), in their theory of self-construal, also demonstrate cultural variation in self-construal influences self-system structures, self-in-relation-to-other schema and internal attributes. Therefore, this thesis believes that an important cultural distinction that influences the nature of the conceptual self is the distinction between an independent versus an interdependent orientation.

Conway conceptualizes the working self to be comprised of a motivational hierarchy of goals and sub-goals that operate to constrain cognition, and ultimately behavior, into effective ways of operating in the world (Conway \& Pleydell-Pearce, 2000). Markus and Kitayama argue that the culturally appropriate self dictates cognition and behavior enabling it to be culturally appropriate. They theorize that goals and motivations are influenced by the nature of the self-schema. The goals and motivations of the independent self are to be unique, express the self, realize internal attributes and promote own goals, whereas the goals and motivations of the interdependent self-construal are to belong, fit-in, occupy one's proper place, engage in appropriate action and promote other's goals. Based on the clear association between these two theoretical approaches, this thesis theorizes that cultural variation in autobiographical remembering is the result of cultural variation in self impacting not only on the conceptual self but also on the working self. Specifically, this thesis hypothesizes that Conway's working self is comprised of both an autonomous and relatedness hierarchy of goals and sub-goals that function to shape cognition and behavior into culturally appropriate, expected and functional ways of operating in the world. 
As shown in Figure 4.1, Conway (2005) conceptualizes the autobiographical knowledge base to be organized by theme (such as relationship theme, work theme, family theme, etc.). He claims that the life story (a level of the autobiographical knowledge base) is a component of the conceptual self and contains factual and evaluative knowledge about the individual, self-related information that can "divide and separate the self into several different selves" (p. 608), and knowledge about goals, others, locations, activities and evaluations, that were common to that period. Additionally, Woike et al. (1999) claim that motives, goals and desires shape the organization of autobiographical memory. Trafimow et al. (1991) have demonstrated convincing support for the notion that relatedness/interdependent and autonomous/independent cognitions are stored in separate locations. Various linguistic studies (e.g., Ross et al., 2002), using samples of bicultural individuals, have illustrated that collectivistic and individualistic cognitions may be stored in separate knowledge structures. Uniting these theoretical stances and empirical findings, this thesis proposes that the autobiographical knowledge base is comprised of an autonomous/independent 'area' and a relatedness/interdependent 'area'.

According to Wang and Conway (2004) the self is instrumental in the retrieval of autobiographical memories, and autobiographical memories function to develop, express and maintain the culturally appropriate self. Therefore, this thesis assumes that the cultural distinction in self also impacts on the retrieval of autobiographical memories. It is hypothesized that everyday memories are integrated into either the autonomous or relatedness area of the autobiographical knowledge base and thus, following Conway's (e.g., Conway \& Pleydell-Pearce, 2000) rationale, have connections to associated lifetime periods and other general events that will be predominantly either relatedness or autonomous in both content and structure. This allows for elaboration of the memories in culturally sanctioned ways. Hence, when everyday memories enter awareness, as shown in Figure 4.2, they are compromised of specific information about the event, general information about the lifetime period and abstracted information about the type of event in general. It is suggested that this information will reflect either autonomy (Figure 4.2.1.) or relatedness (Figure 4.2.2) depending on where the memory was integrated and hence, is currently situated. Such a suggestion fits with Wang and Conway's assumed bidirectional relationship between autobiographical memory and the self. Specifically, "a focus on autonomy versus relatedness in the self at the macro cultural level may affect the content and 
organization of individuals' autobiographical memory" (p. 914), and in turn, the nature of autobiographical memories reaffirm the self as an autonomous or relational self - the culturally appropriate self.

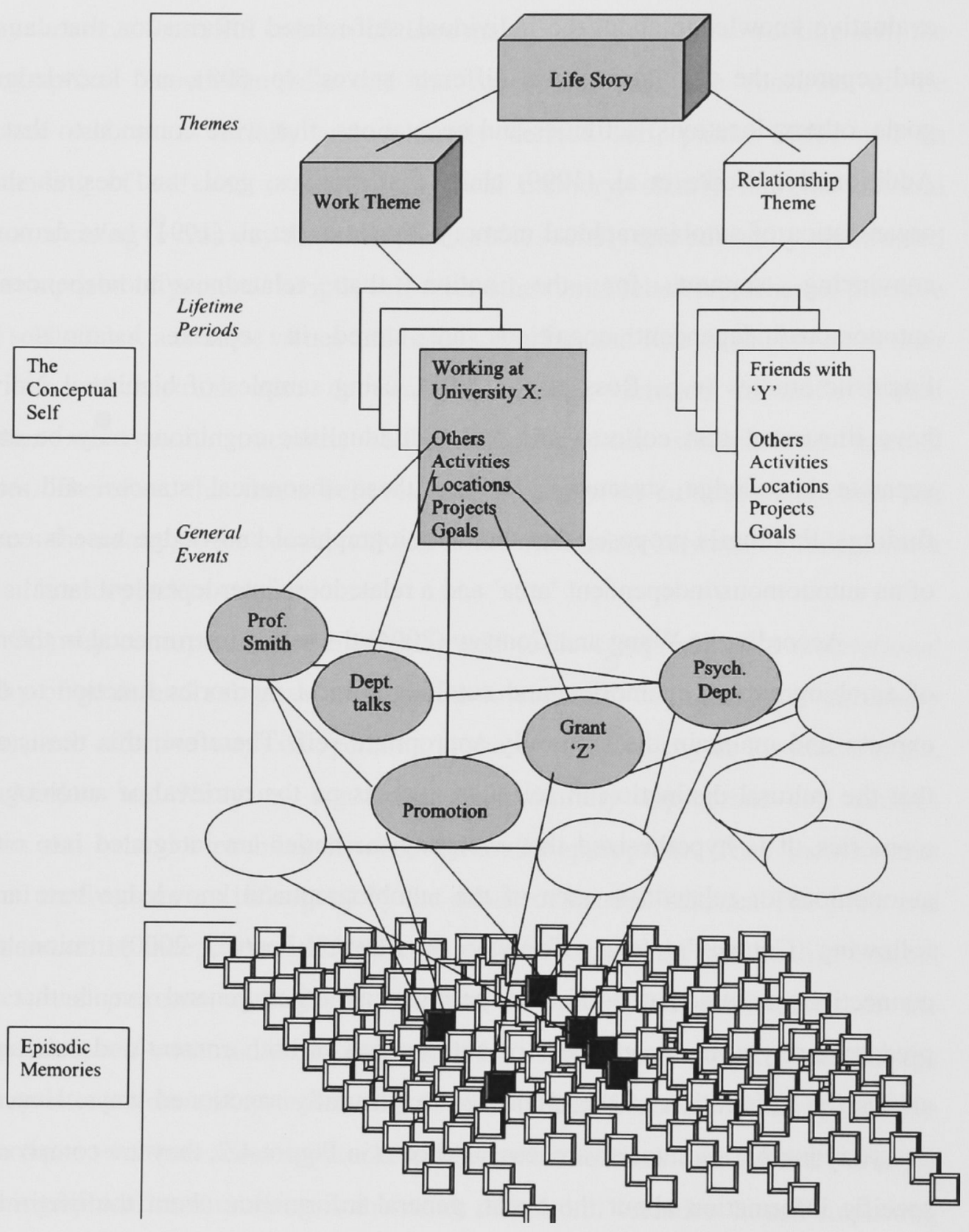

Figure 4.1 Knowledge structures in autobiographical memory. Taken from Conway, M. A. (2005). Memory and the self. Journal of Memory and Language, 53, 594-628. Copyright 2005 by Elsevier Inc. Reprinted with permission. 


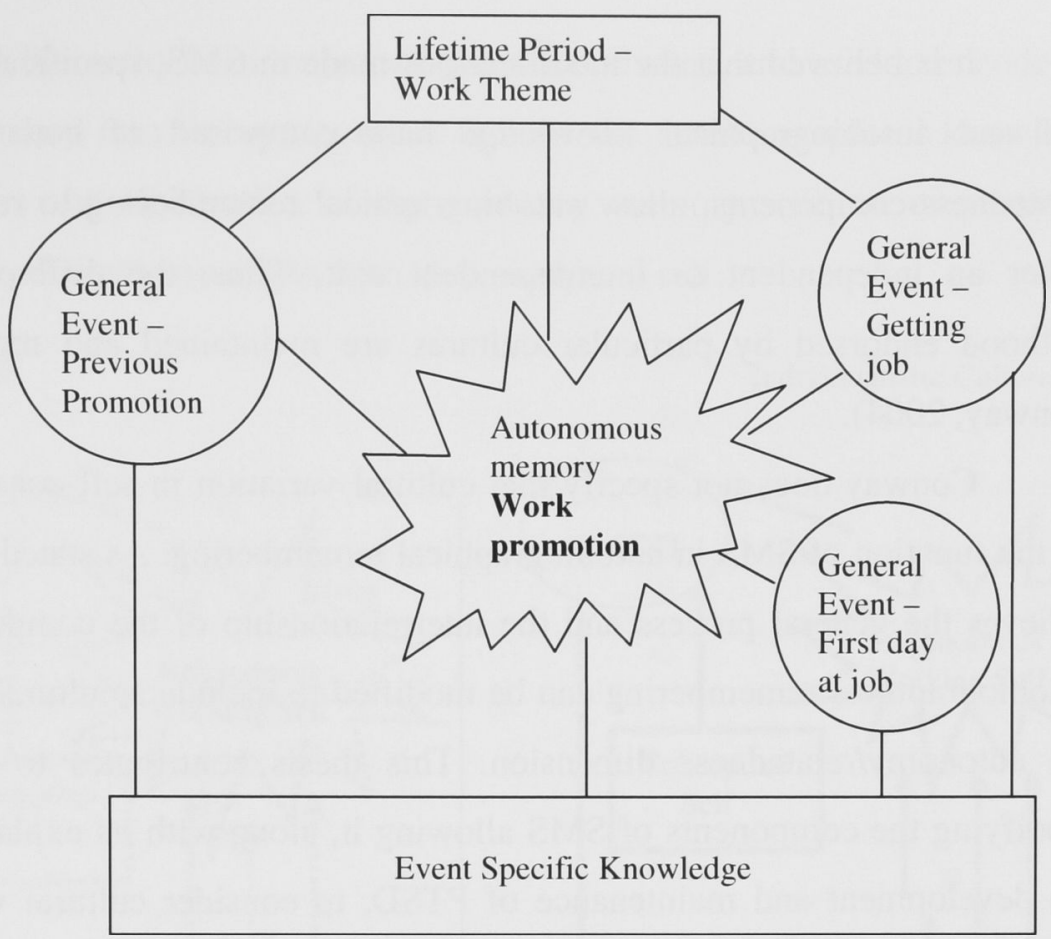

Figure 4.2.1 Retrieval of autonomous autobiographical memories.
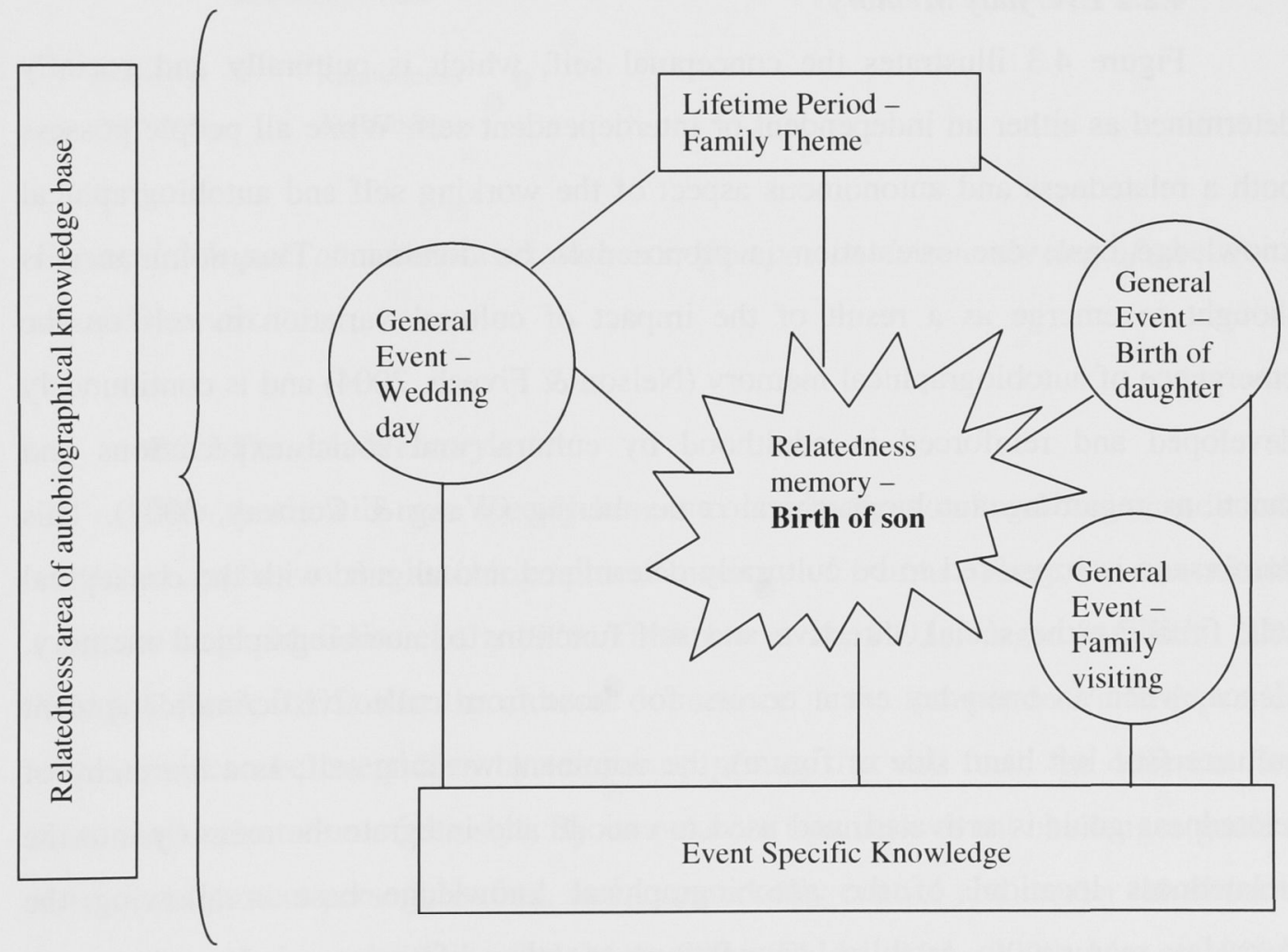

Figure 4.2.2 Retrieval of relatedness autobiographical memories. 
It is believed that the modifications made to SMS, specifically SMS' working self and autobiographical knowledge base comprised of both autonomous and relatedness components, allow autobiographical remembering to reaffirm the self as either an independent or interdependent unit. Thus, the different approaches to selfhood endorsed by particular cultures are maintained and regulated (Wang \& Conway, 2004).

Conway does not specify that cultural variation in self-construal may impact on the function of SMS in autobiographical remembering. As stated above, this thesis believes the general process and the interrelationship of the components of SMS in autobiographical remembering can be modified to include a cultural distinction along the autonomy/relatedness dimension. This thesis contributes to the literature by modifying the components of SMS allowing it, along with its explanations regarding the development and maintenance of PTSD, to consider cultural variations in selfconstrual, and also by testing these modifications empirically.

\subsubsection{Everyday Memory}

Figure 4.3 illustrates the conceptual self, which is culturally and socially determined as either an independent or interdependent self. While all people possess both a relatedness and autonomous aspect of the working self and autobiographical knowledge base, one orientation is proposed to be dominant. This dominance is thought to emerge as a result of the impact of cultural variation in self on the emergence of autobiographical memory (Nelson \& Fivush, 2004) and is continuously developed and reinforced in adulthood by cultural and social expectations and sanctions regarding autobiographical remembering (Wang \& Conway, 2004). This dominance is suggested to be culturally determined and aligned with the conceptual self, fulfilling the social, directive and self functions of autobiographical memory. Hence, when an everyday event occurs, for those from collectivistic/interdependent cultures (see left hand side of figure), the dominant working self, as a hierarchy of relatedness goals is activated, and used to encode and integrate the memory into the 'relatedness location' of the autobiographical knowledge base - allowing the everyday memory to establish connections to other lifetime periods and general events that foreground relatedness, resulting in culturally appropriate elaboration. This process aligns with the conceptual self. In contrast, for those from individualistic/independent cultures (see right hand of the figure), the dominant 
working self, as a hierarchy of autonomous goals is activated, and used to encode and integrate the memory into the autonomous area of the autobiographical knowledge base, allowing for the elaboration of the everyday memory with an emphasis on autonomy. Again, this process aligns with the conceptual self.

Collectivistic Culture

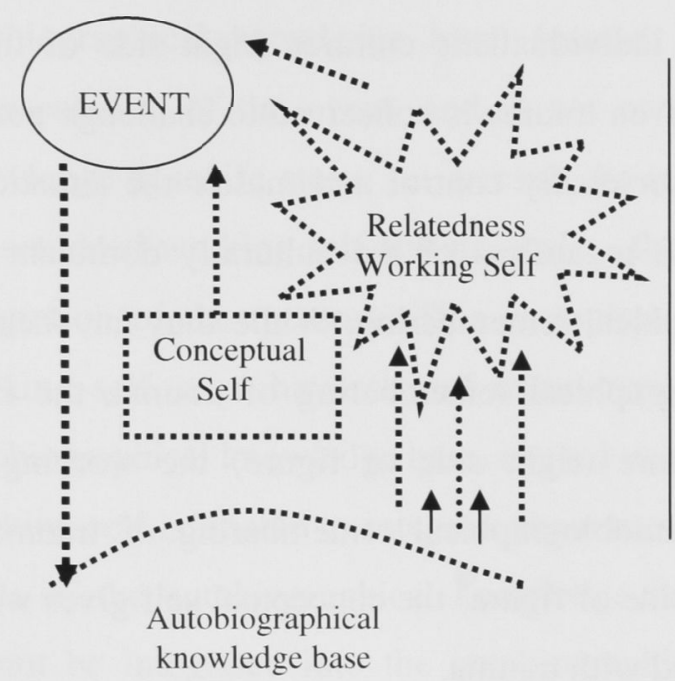

$\begin{array}{ll}\cdots \ldots . . . & \text { Relatedness } \\ & \text { Autonomous }\end{array}$
Individualistic Culture

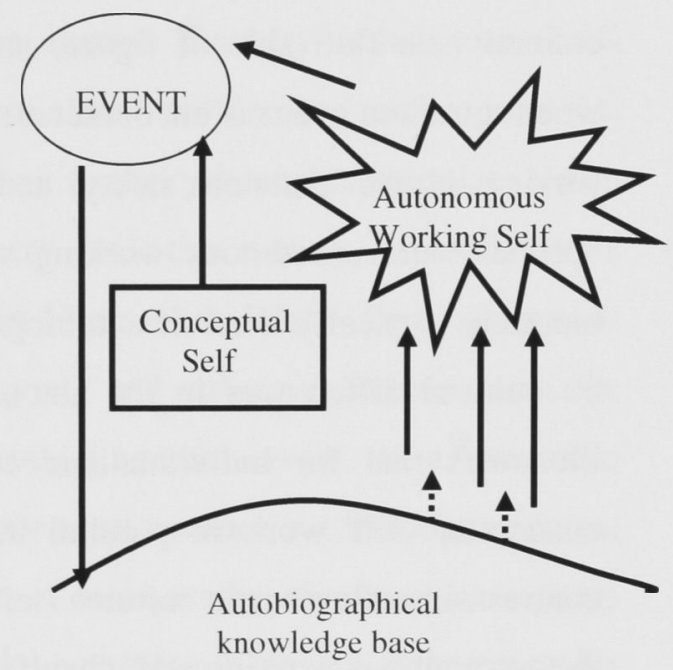

Figure 4.3 Impact of cultural variation in self on personal remembering of an everyday event.

\subsubsection{Trauma Memory}

This thesis will now specify how it conceptualizes cultural variation in selfconstrual applies to the autobiographical memory of trauma. There is accumulating evidence (e.g., McGuire et al., 1978; Trafimow et al., 1991; Wagar \& Cohen, 2003; Wang \& Ross, 2005) that the cultural dominance in the independent/interdependent distinction can be overridden by temporary primes that call forth the different (less dominant) orientation of the self. By its nature a traumatic event challenges goals to survive, to protect personal safety, and to personally control and master the situation (i.e., autonomous goals) (e.g., Dalgleish, 2004; Ehlers et al., 2000; Meares, 2004). Applying this observation to the empirical evidence indicating temporary primes can call forth the non-dominant orientation of self; this thesis predicts that a traumatic event will activate an autonomous goal hierarchy or working self regardless of 
dominant self orientation. From an evolutionary perspective, it seems fitting to suggest that across all cultures, humans, when faced with trauma, fight for personal survival. Therefore, it is thought that there will be registration of the trauma memory as autonomous and in order to maintain an autonomous sense of self there will be a universal foregrounding of autonomy in remembering traumatic events. Figure 4.4 illustrates the thesis' prediction in this regard. As the figure demonstrates in both collectivistic (left side of figure) and individualistic cultures (right side of figure) when a trauma event is encountered, given trauma is conceived to challenge goals to survive, protect personal safety, and personally control and master the situation, it "primes" an autonomous working self, regardless of the culturally dominant goal hierarchy typically used in autobiographical remembering. While thus far, there are no cultural differences in the autobiographical remembering of trauma, the figure illustrates that for individualistic culture (right side of figure) the working and conceptual self work in parallel in autobiographical remembering of trauma. In contrast, in collectivistic cultures (left side of figure) the conceptual self gives way to the autonomous working self when faced with trauma.

Collectivistic Culture

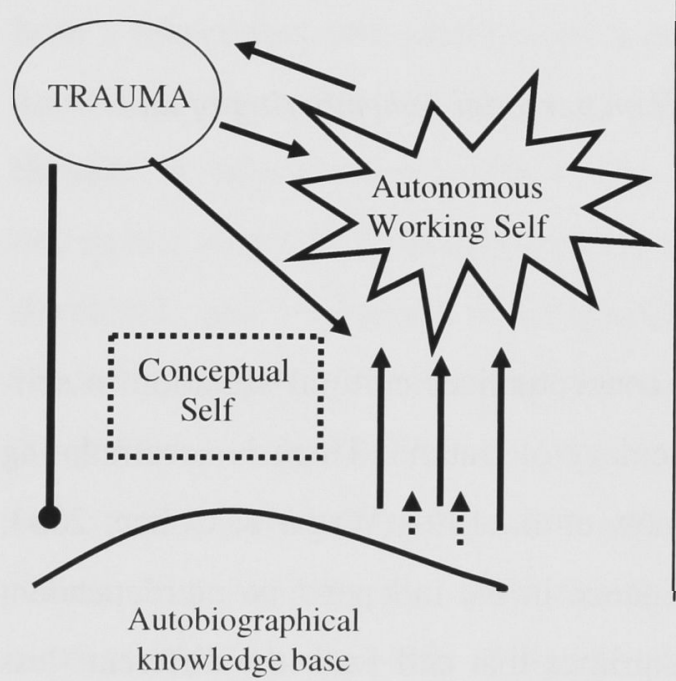

Individualistic Culture

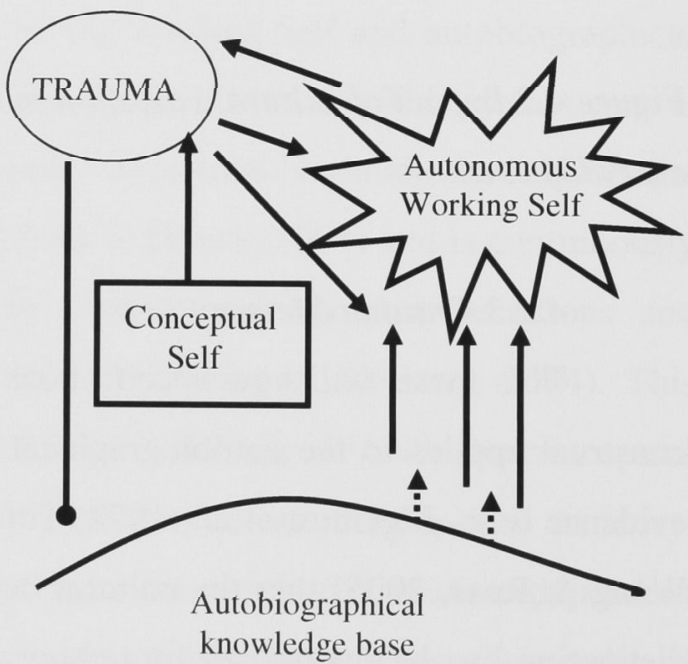

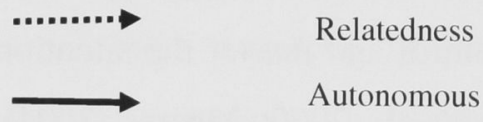

Figure 4.4 Impact of cultural variation in self on personal remembering of a trauma event. 


\subsubsection{Integration of Trauma Memory and Psychological Adjustment}

Conway and Pleydell-Pearce argue that traumatic experiences present a "threat to current plans and goals to which the working self cannot adapt" (p. 281). Trauma violates or contradicts active goals, which might otherwise have been used to integrate the experience with the knowledge base. Hence, there are no currently active goals that can be made use of to mediate integration of the trauma memory into the autobiographical knowledge base. Instead, the trauma memory remains an ESK representation of the event, and is not contextualized within the autobiographical knowledge base/life story. As a result the trauma memory becomes associated with the activated working self and its goals - the very goals that were to be used for the integration of the memory. This is depicted in Figure 4.4. While the autonomous working self, as a hierarchy of autonomous goals and sub-goals drawn from the autobiographical knowledge base, is used to encode the trauma event, the autonomous working self is unable to integrate the trauma memory because it contradicts current goals of the autobiographical knowledge base. Consequently, the trauma memory cannot be integrated into the autobiographical knowledge base but rather remains ESK associated with the goals that were to be used in this integration.

According to SMS, how well people adjust in psychological terms to a trauma depends on the degree that they are able to make use of their existing goals to integrate the traumatic event with their autobiographical knowledge base and the conceptual self. Conway and Pleydell-Pearce (2000) argue that some trauma survivors are unable to achieve this integration of the trauma memory. They argue that nonintegration of the trauma memory results in PTSD intrusion symptoms because the trauma ESK remains highly available as it is triggered by activation of the goals of the working self, which, they presume, to be to some extent always activated. They suggest that it is not until the current goal structure itself changes that intrusions of the memory decrease. Alternatively, excessive availability of the ESK of the trauma memory can be reduced if the trauma memory is linked to contextualizing autobiographical knowledge, such as lifetime periods and general events, as accessibility of the trauma memory would then be managed by the structure of autobiographical knowledge and by the influence of control processes in modulating and inhibiting access to the knowledge base (Conway \& Pleydell-Pearce, 2000).

SMS aims for self-coherence (Conway, 2005). Conservatism is thought to be central to working self goal structure as altering one goal (even successfully) is both 
cognitively and affectively costly as it translates into consequences for many other goals. Furthermore, Conway claims that during "a period of goal-change the self may be more vulnerable to de-stabilizing influences and less capable of operating effectively [in] the world" (p. 597). In response to this, in order to integrate the trauma memory into the life story, self-concept and self-knowledge without altering goals, SMS may alter the trauma memory by lowering the accessibility of these memories of the events or even distorting the memories to allow the trauma memory to match the goal structure. Another common possibility is that the survivor's selfconstruct/conceptual self is altered (Conway, 2005). In support of this, Conway notes that many trauma survivors comment that they are a "different person" following trauma. For example, a trauma survivor may report that they have lost control, are unable to cope or define themselves by their trauma.

This thesis synthesizes SMS with Markus and Kitayma's (1991) theory of cultural differences in self-construal by suggesting that where change in the conceptual self occurs, it will be based on goals consistent with existing goals of the conceptual self (self-construal). Specifically, change in the conceptual self (selfconstrual) can occur when goals activated by the trauma event (predicted to be autonomous goals) match existing goals in the person's goal hierarchy. Therefore, it is suggested that in interdependent cultures, because there is a general lack of congruence between goals associated with memories for trauma (i.e., autonomous goals of personal control, agency, autonomy, etc.) and the goals of the conceptual self (i.e., relatedness goals of meeting social obligations and responsibilities, maintaining group harmony, not standing out, and the little emphasis on personal control, etc.), then there is less need to integrate the trauma memory with the conceptual self, and thus, problematic psychological adjustment is less likely to be associated with selfchange.

Additionally, SMS privileges self-consistency and self-coherence, as do other PTSD theories (e.g., Berntsen \& Rubin, 2007). Suh's (2000) theory of selfconsistency demonstrates that self-consistency needs are culturally variable. Specifically, while a need for self-coherence is believed to be critical for those from independent cultures, it seems to be not dominant in interdependent cultures where the focus is on the social context rather than on the person, and people from these cultures are much more capable of flexibility between social roles and tolerant of differences in their self in these roles. Markus and Kitayama (1991) theorize that the 
self is defined in culturally specific ways. In independent cultures the self is believed to be defined by a set of internal attributes that are consistent across time and context, whereas in interdependent cultures relationships with others in specific contexts define the self. Lu et al. (2001) suggests that in independent cultures crucial selfrepresentations are held within the individual, while for interdependent cultures selfrepresentations tend to be held in social relationships rather than within the individual. Therefore, in interdependent cultures self-representations remain bound to the social context and self-knowledge is generally not abstracted across situations but remains specific to the focal context.

The thesis has applied both Suh's theory of self-consistency and Markus and Kitayama's theory of self-construal to SMS, to predict that while those from independent cultures may have changes in their self-concept/conceptual self (such as centered on being a trauma survivor/victim or self-change since the event) to maintain and achieve self-consistency, those from interdependent cultures will not adopt such a change to the conceptual self as self-definition is derived from others, and is constrained to specific time and contexts. Specifically, a trauma survivor from an independent culture may adopt the role of trauma victim/survivor and its associated characteristics (e.g., weak, incapable, etc.) as self-consistency is needed, and the self is defined by such internal characteristics. In contrast, a trauma survivor from an interdependent culture tends not to adopt the role of trauma victim/survivor, and its associated characteristics (e.g., weak, incapable, etc.), as self-consistency is not required and the self is defined by the social context (e.g., I am a mother, I am a teacher, etc.), which is less likely to be changed by trauma. Such internal characteristics and trauma self-representations remain bound to the trauma context and are generally not abstracted across situations but remain specific to the focal context.

\subsection{Summary of Cultural Modifications made to SMS}

Up to this point the cultural theories of self-construal have been synthesized with SMS to expose SMS to the notion of cultural differences in self. The thesis suggests that all components of SMS are influenced by cultural differences in self. First, while SMS posits that the conceptual self is comprised of personal scripts, 'selfwith-other units' relational schema, possible selves and internal attributes, Markus 
and Kitayama (1991) theorize that cultural variation in self-construal influences, and often governs self-system structures, self-in-relation-to-other schema and internal attributes. Therefore, the thesis proposes cultural differences in independent versus interdependent self orientation moderates the nature of the conceptual self.

Second, while SMS conceptualizes the working self to be comprised of a motivational hierarchy of goals that operate to constrain cognition, and ultimately behavior, into effective ways of operating in the world, Markus and Kitayama argue that the culturally appropriate self dictates cognition and behavior enabling it to be culturally appropriate, and goals and motivations are influenced by the nature of the self-schema. Therefore, the thesis hypothesizes that the working self is comprised of both an autonomous and relatedness hierarchy of goals and sub-goals that function to shape cognition and behavior into effective (including culturally effective) ways of operating in the world.

Third, SMS conceptualizes the autobiographical knowledge base to be organized by theme and contains knowledge about the self and others, and information that can "divide and separate the self into several different selves" (p. 608). Theorists (e.g., Wang \& Conway, 2004; Woike et al., 1999) claim the self, motives and goals shape the organization of autobiographical memory. Therefore, the thesis claims that the autobiographical knowledge base is comprised of both an autonomous and relatedness 'area'. Following on from this, given Wang and Conway (2004) posit that the self is instrumental in the retrieval of autobiographical memories and memories are integrated into either the autonomous or relatedness location in autobiographical memory, the thesis suggests the cultural distinction in self also impacts on the retrieval of autobiographical memories allowing for the re-affirmation of the conceptual self.

Drawing this together, the thesis predicts the following regarding the autobiographical remembering of everyday events. It is posited that the conceptual self is culturally and socially determined as either an independent or interdependent self. While all people possess both a relatedness and autonomous aspect of the working self and autobiographical knowledge base, one is proposed to be dominant, depending on culture and aligning with the conceptual self. Hence, when an everyday event occurs, the dominant working self and its associated hierarchy of goals is activated, and used to encode and integrate the memory into the appropriate 'location' 
of the autobiographical knowledge base, enabling culturally appropriate elaboration and reaffirmation of the conceptual self.

In terms of the trauma memory, there is evidence (e.g., Trafimow et al., 1991) that the cultural dominance in the independent/interdependent distinction can be overridden by temporary primes that call forth the different orientation of the self and theorists (e.g., Dalgleish, 2004) posit that a traumatic event by its nature challenges goals to survive, protect personal safety, and personally control and master the situation. Therefore, the thesis hypothesizes that a traumatic event by its nature will activate an autonomous goal hierarchy or working self. It is thought that in order to maintain an autonomous sense of self there will be a universal foregrounding of autonomy in remembering traumatic events.

According to SMS, how well people psychologically adjust post-trauma depends on the degree that they are able to make use of their existing goals to integrate the traumatic event with their autobiographical knowledge base and the conceptual self. One way to integrate the trauma memory is to alter the survivor's self-construct/conceptual self. The thesis therefore predicts that in posttrauma adjustment, where change in the conceptual self occurs it will be based on goals consistent with existing goals of the conceptual self. Therefore, it is predicted that in interdependent cultures, because there is a general lack of congruence between goals associated with memories for trauma (autonomous) and the goals of the conceptual self (predominantly relatedness), then problematic psychological adjustment is less likely to be associated with self-change. Additionally, synthesizing SMS' emphasis on self-consistency with Suh's (2000) theory of self-consistency and Markus and Kitayama's (1991) assumptions regarding self-definition, the thesis predicts that while those from independent cultures with poorer psychological adjustment may have trauma-centered changes in their conceptual self to maintain self-consistency, there will be no relationship between trauma-centered self-definition and psychological adjustment for those from interdependent cultures, as self-definition is derived from social roles and trauma-centered self-definition will be constrained to a specific time and context. 


\subsection{Processes other than Autobiographical Memory Implicated in PTSD Models}

The thesis will now proceed to amalgamate the theory of self-construal with processes, other than autobiographical memory, thought to be important in understanding the development and maintenance of PTSD.

\subsubsection{Appraisals}

Ehlers and Clark (2000) suggest a reciprocal relationship between the nature of the trauma memory and the appraisals of the trauma and the post-trauma events. For those with poor post-trauma psychological adjustment, the recall of the trauma memory tends to be biased by appraisals, and people selectively retrieve information and memories that is consistent with their appraisals posing an on-going threat, which can be either internal (i.e., to the self) or external. Ehlers and Clark have identified a wide range of appraisals, that include appraisals that focus on the self and one's actions, autonomy and permanent change (e.g., "I was weak", "I deserved for this to happen", "I am incompetent, inferior, unworthy", "I am permanently damaged"), while other appraisals focus on others, alienation and other's reactions (e.g., "Others can know that I am a trauma victim", "I will always be alone and no one understands", "Others think I cannot cope"). Ehlers and Clark posit that appraisals centered round the self may maintain PTSD as survivors continue to perceive current situations as threatening and dangerous, as they perceive themselves as inadequate and unable to cope in current situations, and retrieve information and memories that confirm this inadequacy. They suggest appraisals about others and interpersonal relationships may maintain PTSD because survivors have on-going negative appraisals of interactions with others, and thus, may withdraw from social interactions and are thus, less likely to receive social support and to correct negative beliefs about themselves and others (Ehlers et al., 2000).

Mesquita and Walker (2003) demonstrate both theoretically and empirically that cultural differences in self-construal impact on the way in which events, situations and life encounters are appraised. They posit that cultural differences in self-construal "facilitate and render desirable certain appraisals of events, while making the occurrence of others less likely and less valued" (p. 784) and thus, such differences in self "foster culture-specific appraisal tendencies that are reflected in culturally distinct patterns of emotional experience" (p. 784). According to Markus 
and Kitayama's (1991) theory, individualistic cultures aim to attribute events as under personal control, agency and responsibility, while Mesquita and Walker posit that for collectivistic cultures, personal agency has very limited applicability, rather the interdependence of an individual and their social environment is stressed. As outlined in Chapter 3, there is empirical support for this argument. Additionally, such cultural differences in appraisals have been found (e.g., Mesquita \& Walker, 2003; Sato, 2001) to impact on affective responses with individualistic cultures experiencing less positive affect and possible poor psychological adjustment when they feel diminished autonomy and personal control in a situation, whereas for collectivistic cultures this seems not to be the case. Sato (2001) suggests in interdependent cultures, alienation and isolation can result in poor psychological outcomes.

Combining the literature indicating cultural differences in self-construal impact on appraisals and subsequent affective responses with Ehlers and Clark's appraisal model, this thesis, firstly, hypothesizes that while the nature of the trauma memory will foreground autonomy (following the rationale set out above); the trauma associated appraisals will reflect the conceptual self. That is, the level of personal agency/autonomy in the trauma memory will be more important to individualistic cultures, than those from collective cultures. Specifically, individualistic cultures will try to attribute the trauma event as under personal control, whilst in collectivistic cultures the interdependence of the survivor and their social environment will be stressed. In collectivistic cultures, personal agency in the trauma will have very limited applicability. Therefore, Ehlers and Clark's identified appraisals that focus on the self and one's actions, autonomy and permanent self-change are suggested to be more prominent in individualistic cultures than collectivistic cultures, while appraisals that focus on others, alienation and other's reactions will be more salient in collectivistic cultures than in individualistic cultures.

Secondly, it is hypothesized that such cultural differences in trauma appraisals will impact on affective responses and psychological adjustment. It is predicted that self-focused trauma appraisals will have greater impact on posttrauma psychological adjustment of trauma survivors from individualistic cultures, whilst other-focused trauma appraisals will have a greater impact on the psychological adjustment of those from collectivistic cultures. It is believed that trauma survivors from individualistic cultures will experience more positive affect and better psychological adjustment (i.e., less PTSD symptoms) when they appraise personal control during and after the 
trauma, whereas collectivistic cultures will experience more positive affect and better psychological adjustment (i.e., less PTSD symptoms) when factors such as the interdependence of the survivor and their social environment are emphasized in trauma associated appraisals.

Thirdly, the thesis hypothesizes that PTSD will be maintained in individualistic cultures when the recall of the trauma memory is biased by appraisals about lack of autonomy, as these survivors will selectively retrieve information and memories that are consistent with these appraisals, posing an on-going threat to the conceptual self. Specifically, PTSD survivors from individualistic culture continue to perceive current situations as threatening and dangerous, as they perceive themselves as inadequate and unable to cope in current situations, and retrieve information and memories that confirm this inadequacy. This continually threatens the conceptual self, which is culturally required to operate in effective ways in the world and with others. PTSD will be maintained in collectivistic cultures when the recall of the trauma memory is biased by appraisals that lack relatedness, as these survivors will selectively retrieve information and memories that are consistent with these appraisals, posing an on-going threat to the conceptual self. Appraisals about others and interpersonal relationships have on-going negative appraisals of interactions with others, alienation, failing in social roles, which continually threaten the conceptual self; which is culturally required to operate in effective ways in the world and with others.

\subsubsection{Identity}

Briefly, Berntsen and Rubin's (2007) centrality of trauma approach suggests that trauma can become central to identity because memories of the trauma are highly accessible and easily evoked, and hence, the trauma event becomes perceived over time as "a major causal agent" and "thus a highly salient turning point in the person's life" (2006, p. 221). Typically, turning points are culturally expected transitional events that provide self-definition or change in self-definition through role change. The perception of the trauma event as a turning point together with the requirement for a self-consistency results in the role of trauma victim or survivor, becoming salient and the important component of identity. However, as in SMS instance, selfconsistency needs are believed to be culturally variable (Suh, 2000) thus, this thesis 
suggests trauma-centered identity is more likely for those from individualistic cultures than for those from collectivistic cultures.

Berntsen and Rubin posit that maintenance of this trauma-centered identity is thought to be the consequence of "culturally sanctioned role transitions" and "personal identification with the social roles that are culturally expected" (2007, p. 420). Such an assumption would predict stronger maintenance of a trauma related self-concept in independent cultures. In independent cultures promoting the self by means such as publicizing the individual's life story, personal identity and uniqueness, master or lack of mastery are accepted, valued and culturally sanctioned (e.g., Wang, 2001). Summerfield (2004) claims that in independent cultures personhood emphasizes the deep, hidden, private self, and emotion and vulnerability following trauma. Therefore, the social role of trauma survivor/victim aligns with independent cultural expectations of the self, personal identity and the individual life story. However, in interdependent culture discussing the individual's life story, personal identity and uniqueness may be viewed as abnormal, immature or arrogant, that is, culturally inappropriate (Sato, 2001; Suh, 2000). These cultures downplay the independent self in favor of the communal, relational self (Nelson \& Fivush, 2004; Wang, 2001) and collective activities are often valued over a unique life story (Wang, 2001). Therefore, the social role of trauma survivor/victim contradicts the interdependent cultural expectations of a communal self.

\subsubsection{Transformation of Meaning}

A number of the socio-cognitive theories of PTSD (e.g., Dalgleish, 2004; Ehlers \& Clark, 2000; Horowitz, 1986; 1999; Janoff-Bulman, 1992) propose that trauma impacts on survivors' belief systems. The models claim that the assumptions originally held about the self, others and world are shattered by the trauma resulting in a transformation in meaning (Dalgleish, 2004; Janoff-Bulman, 1992).

In Markus and Kitayama's (1991) self-construal theory it is suggested that the cultural distinction in independence versus interdependence orientation impacts on beliefs, thoughts, feelings and behavior regarding self, others and the world. Therefore, this thesis proposes that cultural variation in self impacts on transformation of meaning. It is suggested that trauma survivors from independent cultures will focus on the self and private aspects of the self, while trauma survivors from interdependent cultures will focus on interdependence of the self. 


\subsection{Summary of Thesis' Conceptualization of how Cultural Differences in Self- Construal Moderate Processes other than Autobiographical Memory Implicated in PTSD Models}

The thesis hypothesized how the cultural distinction in autonomous/relatedness orientation in self impacts on the autobiographical remembering of trauma. The thesis then amalgamated the theory of self-construal with processes, other than autobiographical memory, thought to be important in understanding the development and maintenance of PTSD.

While Ehlers and Clark's (2000) appraisal model suggests a reciprocal relationship between the nature of the trauma memory and the appraisals of the trauma which can pose an on-going threat to self, Mesquita and Walker (2003) demonstrate both theoretically and empirically that cultural differences in selfconstrual impact on the way in which events, situations and life encounters are appraised and on associated affective responses. Therefore the thesis hypothesized that trauma associated appraisals will reflect the conceptual self. That is, the level of personal agency/autonomy in the trauma memory will be more important to individualistic cultures and their subsequent psychological adjustment, than those from collective cultures. Those from collectivistic cultures will appraise trauma in terms of the interdependence of the survivor and their social environment and such appraisals will moderate psychological adjustment.

Berntsen and Rubin's (2007) centrality of trauma approach suggests that trauma can become central to identity and maintained as a result of "culturally sanctioned role transitions" and "personal identification with the social roles that are culturally expected" (2007, p. 420). It was suggested that the social role of trauma survivor/victim aligns with independent cultural expectations, while in interdependent cultures the social role of trauma survivor/victim contradicts the interdependent cultural expectations of a communal self.

Finally, a number of the socio-cognitive theories of PTSD (e.g., Dalgleish, 2004; Ehlers \& Clark, 2000) propose that trauma impacts on survivors' belief systems. In Markus and Kitayama's (1991) self-construal theory it is argued that the cultural distinction in independence versus interdependence orientation impacts on beliefs systems. Therefore, this thesis proposed that cultural variation in self impacts on transformation of meaning. 


\subsection{Studies}

Five studies have been developed to empirically test the above conceptual framework. The framework is revisited and discussed more thoroughly in the introduction of the individual studies, allowing for precise hypotheses to be generated. The first series of studies (Studies 1 and 2) focus on the trauma memory in a nonclinical sample. Specifically, the first study explores how cultural differences in self impact on autobiographical remembering of trauma and explores the impact of cultural differences in self on personal identity, self-definition and psychological adjustment. This study initiates an investigation into the framework outlined above, as it tests whether the cultural differences apparent in the everyday memory, are absent in the autobiographical remembering of trauma, as trauma by definition primes an autonomous hierarchy of goals. Secondly, it explores whether the cultural distinction in self is influential in maladjustment to trauma in terms of autobiographical memory and identity. Thus, this first study examines the cultural modification to SMS. The second study uses Ehlers and Clark's (2000) framework to explore the impact of cultural differences in self on appraisals and the subsequent impact of this on psychological adjustment. The second series of studies (Studies 3-5) extends the ecological validity and clinical implications of Series 1, and rectifies any methodological flaws in Series 1. Studies 3, 4 and 5 investigate the research questions in a clinically relevant sample, comprised of trauma survivors with and without PTSD from independent and interdependent cultures.

Narratives and self-defining memories will be used to empirically test the predictions derived from the conceptual framework. Narratives are a common measure of autobiographical memory (e.g., Fivush \& Haden, 2003; Freeman, 2007; McAdams et al., 2006; Wang \& Conway, 2004) and are the most common form of measuring cultural differences in autobiographical memory (e.g., Wang, 2001; Wang \& Conway, 2004). Additionally, narratives are routinely used in trauma research (e.g., Dunmore, Clark, \& Ehlers, 2001; Ehlers, Clark, et al., 1998; Harvey \& Bryant, 1999; Kenardy et al., 2006; O’ Kearney, Speyer, \& Kenardy, in press).

This thesis will apply the approach to analyzing narratives as a measure of cultural differences in autobiographical remembering, as used by other cultural researchers (Han et al., 1998; Wang, 2001; Wang \& Conway, 2004; Wang \& Leichtman, 2000; Wang, Leichtman, \& White, 1998), to the trauma memory. 
Therefore, the trauma narratives will be coded for theme (classifying the memory narrative as either autonomous or relatedness themed), autonomous orientation (a measure of the emphasis of autonomy in the memory narrative), other/self ratio (a measure of the degree of providing non-egotistic memories), and interaction scenario (number of instances that involved social interactions or group activities). Furthermore, Wang and Conway (2004) found that Chinese participants made significantly more reflective comments than American participants suggesting those from collectivistic culture more "frequently draw upon past events to convey moral messages, making reflective comments on how a memory event had taught them about life or the world" (p. 917), than individualistic cultures. Han et al., (1998) and Wang and Conway (2004) have demonstrated that those from collectivistic cultures are less likely to provide specific (i.e., event happened at a particular point in time) memories than individualistic cultures. Wang and Conway (2004, p. 912) suggest specificity is required in individualistic cultures to "serve an important means to differentiate the self from others, thereby re-affirming the self as an autonomous self". Therefore, specificity and reflective comment will also be coded in the trauma memories.

The trauma narratives will also be used to explore trauma-associated appraisals. Following Ehlers' approach (e.g., Dunmore et al., 2001) the trauma narratives will be analyzed using her coding system for the appraisals of mental defeat (loss of sense of psychological autonomy during the trauma), control strategies (strategies taken to minimize the impact of the trauma), alienation (sense of being socially isolated) and permanent change (sense of self and life change for the worse).

A specific type of autobiographical memory is the self-defining memory. Bauer and McAdams (2004) claim that people's intentions and value orientations can be discerned in narratives of people's self-defining memories. A self-defining memory is a memory of an event that an individual believes shaped "who I am", and is essential in giving meaning to the self (Singer \& Salovey, 1993). Cultural differences in autobiographical memory content and quality has been demonstrated for memories elicited by simple instructions to recall memories from the past or a particular time in the past (e.g., earliest memory, everyday memory). This thesis aims to contribute to the literature on autobiographical remembering by examining cultural differences in memories that are regarded as self-defining. Because they function to distinguish the person as a unique individual, demonstrating cultural differences in the 
content and structure of self-defining memories would seem to provide powerful support for the inference from earlier work that a cultural conceptual self is critical to regulating autobiographical remembering (Conway et al., 2005). Furthermore, the self-defining memory task has been selected as Sutherland and Bryant (2006) posit the self-defining memory task is a constructive approach to understanding alterations in self-construct and thus, is a constructive approach to investigating autobiographical memories in PTSD because traumatic experiences often alter one's self construct (Berntsen \& Rubin, 2007; Conway, 2005).

\subsection{Conclusion}

The introduction addressed the four objectives stated in Chapter 1. First, Chapter 2 outlined the current prominent socio-cognitive models of PTSD. The importance of autobiographical memory in distinguishing between those with and without PTSD is undeniably central to most of these models. Additionally, other processes such as appraisals, motivation, goals, emotion, and so forth, are important in understanding the etiology and maintenance of PTSD. Second, Chapter 3 explored cultural distinction in the self in terms of an independent and interdependent self. It was demonstrated that although individuals are motivated to achieve both aspects of the self, independence is emphasized in individualistic cultures, whilst interdependence is emphasized in collectivistic cultures. This cultural difference has been found to impact on autobiographical memory and it is in these cultural differences that the culturally emphasized self is reaffirmed. This chapter also reviewed the cultural literature indicating that cultural differences in self influence appraisals, motivation and goals, schema and affective responses. Therefore, given this cultural distinction in self impacts on both autobiographical memory and other processes implicated in our PTSD models, it became obvious that our PTSD models need consider cultural variation in the self. In response to this, the third aim of the introduction was to develop a conceptual framework that accounted for cultural differences in self on autobiographical memory and general processes. The studies developed in this thesis are designed to empirically test this conceptual framework. 
Series 1

Cultural Differences in Autobiographical Memory of Trauma in a Non-Clinical Sample 


\section{CHAPTER 5}

\section{Study 1: Cultural Differences in Autobiographical Memory of Trauma and Retrieval of Self-Defining Memories in a Non-Clinical Sample}

By synthesizing Markus and Kitayama's (1991) theory of cultural differences in self-construal with Conway's (2005) self-memory system (SMS), the conceptual framework (Chapter 4) modified SMS in such a way as to, firstly, enable it to account for observed (e.g., Wang, 2001; Wang \& Conway, 2004) cultural differences in autobiographical remembering of everyday events and, secondly, generate hypotheses regarding how cultural differences in self-construal may operate in autobiographical remembering of trauma. Study 1, comprised of three sub-studies, examines these modifications to SMS. Each sub-study tests one particular hypothesis developed from the conceptual framework. First, cultural distinction in autobiographical remembering applies to everyday but not trauma memories. Second, there is culturally appropriate elaboration of autobiographical memories. Third, the relationship between change in self-definition and disturbed adjustment is culturally dependent.

\subsection{Autobiographical Remembering of Everyday and Trauma Events (Study 1a)}

The conceptual framework predicts that the cultural distinction between autonomy versus relatedness orientation, evident for autobiographical remembering, will not be salient in autobiographical remembering of trauma. Previous findings indicate those from independent cultures are more likely to retrieve autobiographical memories which are; focused on personal experiences (theme of memory) (e.g., Wang \& Conway, 2004); specific (i.e., an event that happened at a particular point in time) (e.g., Han et al., 1998); egotistic (as measured by other/self ratio) (Hyman \& Neisser, 1992; Wang \& Conway, 2004); autonomously oriented (e.g., Wang \& Conway, 2004); contain few reflective comments (Wang \& Conway, 2004); and contain few instances of social interactions or group activities (as measured by interaction scenario) (e.g., Fivush, Haden, \& Adam, 1995; Wang \& Conway, 2004). In contrast, this literature indicates those from interdependent cultures are more likely to retrieve autobiographical memories that are; focused on social events and collective activities (theme) (e.g., Wang \& Conway, 2004); general (i.e., events that took place regularly or on multiple occasions) (e.g., Han et al., 1998); non-egotistic; socially oriented (e.g., 
Wang \& Conway, 2004); contain reflective comments (Wang \& Conway, 2004); and contain substantial number of instances that involve social interactions or group activities (e.g., Fivush et al., 1995; Wang \& Conway, 2004). According to the conceptual framework, these cultural differences are the result of the dominant working self encoding and integrating the everyday autobiographical memory into the suitable 'location' of the autobiographical knowledge base. Such processes allow for culturally appropriate elaboration and retrieval of the autobiographical memory. Consequently, autobiographical remembering aligns with the conceptual self and is therefore instrumental in reaffirming the conceptual self (Wang \& Conway, 2004).

The modified conceptual framework claims trauma by definition will activate an autonomous goal hierarchy or working self. Thus, in order to maintain an autonomous sense of self, there will be a universal foregrounding of autonomy in the remembering of traumatic events. While in the autobiographical remembering of trauma for those from independent cultures the working and conceptual selves are believed to be still aligned, for those from interdependent cultures the conceptual self is thought to give way to the autonomous working self. It is therefore suggested that trauma memories and everyday memories of independent cultures will be similarly autonomously-oriented, while the trauma memories of interdependent cultures will be more autonomously-oriented than the everyday memory. Study 1a tests these predictions by asking Australians and Asians to provide narratives of an everyday and trauma event.

\subsection{Elaboration of Autobiographical Memories (Study 1b)}

The conceptual framework suggests culturally appropriate elaboration and retrieval of the everyday autobiographical memory. Although research (as outlined above) clearly indicates cultural difference in self impacts on autobiographical memory content, the nature and determinants of cultural differences in the quality of autobiographical memory, particularly in the degree of elaboration, are less clear. Some researchers (Conway, Wang, Hanyu, \& Haque, 2005; Wang, 2001) suggest that people from independent cultures are more likely to provide lengthy and highly elaborated autobiographical narratives than people from interdependent cultures, whose autobiographical memories are depicted as brief, routine and less elaborate. There is some evidence to support these suggestions for autobiographical memories 
that focus on individual experiences, personal roles, and emotions (Conway et al., 2005; Wang, 2001; Wang \& Conway, 2004).

The suggestions, however, do not accord with the conceptual framework's assertion regarding culturally appropriate elaboration of autobiographical memories. Additionally, the suggestions do not accord with SMS' functional perspective on autobiographical remembering, which proposes that the quality of the memories helps regulate affect by ensuring a correspondence between acts of remembering and the dominant conceptual self-knowledge (Conway, 2005). For independent cultures correspondence should be achieved by elaborating mainly autonomous aspects of autobiographical memory, while for interdependent cultures congruence should be maintained by elaborating relatedness aspects. There is some indirect evidence to support this view. Mothers from independent cultures emphasize the unique individual when reminiscing with their children focusing on the child's actions and opinions, while Asian mothers downplay the autonomous self in favor of a discussion about social interactions, activities and social norms (Nelson \& Fivush, 2004; Wang et al., 2000). Furthermore, findings (Han et al., 1998; Wang, 2001; Wang \& Fivush, 2005) demonstrate that regardless of culture, people provide both autonomous and relatedness autobiographical memories. These considerations suggest that, rather than a general cultural difference in the elaboration of autobiographical memories, the cultural difference in the degree of elaboration of autobiographical memory depend on the type of information that is retrieved.

Additionally, theoretical approaches (e.g., Conway \& Pleydell-Pearce, 2000; Ehlers \& Clark, 2000) posit poor elaboration of the trauma autobiographical memory. However, the impact of cultural variation in self on the difference in elaboration of trauma and non-trauma memories remains unknown. It is proposed that, as outlined in the conceptual framework, given trauma memories are predicted to be predominately autonomous and have greater cultural acceptance in independent cultures, those from independent cultures will elaborate trauma memories to a greater extent than those from interdependent cultures.

The cultural difference in autobiographical memory content and quality has been demonstrated for memories elicited by simple instructions to recall memories from the past or a particular time in the past. Study $1 \mathrm{~b}$ contributes to the literature on autobiographical remembering by examining cultural differences in memories that are regarded as self-defining. 


\subsection{Self-Definition (Study 1c)}

The framework hypothesizes that the relationship between change in selfdefinition and disturbed adjustment is culturally dependent. According to SMS, the trauma memory is initially non-integrated into autobiographical and self knowledge. Psychological adjustment depends on the degree to which people are able to make use of their existing goals to integrate the traumatic event with their autobiographical knowledge base and conceptual self to achieve self-consistency and self-coherence. When this integration is not achievable a number of solutions are available. One such solution is that over time a sense of integration may be maintained by alteration in the person's self-construct (conceptual self) and goals leading to the development of a self-identity and goals centered on being a victim of trauma or emphasizing selfchange since the event (Conway, 2005).

There is some empirical support for trauma-centered self-change in people with disturbed adjustment to trauma. McNally, Lasko, Macklin, and Pitman (1995) noted that the self presentations of some veterans with PTSD were intimately connected with memories of themselves as servicemen. Importantly, these veterans had difficulties retrieving specific autobiographical memories of positive events and were more likely to recall autobiographical memories of their service experience. Their identity and autobiographical memories had become trauma-centered. Sutherland and Bryant (2006), using a self-defining memory task, found that PTSD participants reported themselves as being more strongly defined or identified by their trauma than those who do not develop PTSD. Further, they found that retrieval of trauma-related self-defining memories was strongly associated with reporting traumarelated personal goals. Byrne, Hyman, and Scott (2001) found, using a sample of female undergraduate students who reported having had a traumatic event, that the degree to which the trauma memory was rated as important for self-understanding was positively related to the severity of symptoms on a PTSD checklist. Berntsen and Rubin $(2006,2007)$ have repeatedly demonstrated a significant positive correlation between the traumatic memory forming a central component of personal identity and self-reported severity of PTSD symptoms. These observations suggest that a significant alteration in self-concept and identity is a potential outcome of trauma.

According to SMS, alongside other models (e.g., centrality of trauma approach, Berntsen \& Rubin, 2007), this self-change is motivated by a need for self- 
coherence and self-consistency. However, as outlined in the conceptual framework, self-consistency needs are culturally variable. The conceptual framework, therefore, predicts that while those from independent cultures may have changes in their selfconcept/conceptual self (such as centered on being a trauma survivor/victim or selfchange since the event) to maintain and achieve self-consistency, those from interdependent cultures will not adopt such a change to the conceptual self as selfdefinition is derived from the social context and trauma-related internal characteristics and self-representations remain bound to the trauma context and are generally not abstracted across situations.

The conceptual framework also outlined a role for culture in the maintenance of trauma identity. The SMS posits that change in the conceptual self will be based on goals consistent with existing goals of the conceptual self. Specifically, change in the conceptual self can occur when goals activated by the trauma match existing goals of the conceptual self. It is thus suggested that for people from collective cultures because there is a general lack of congruence between goals associated with memories for trauma (autonomous) and the goals of the conceptual self (predominantly relatedness) then problematic psychological adjustment may be less likely to be associated with self-change, particularly the development of a self-definition around the trauma. Additionally, as outlined in the conceptual framework, the centrality of trauma approach (Berntsen \& Rubin, 2007) gives importance to the role of social sanctioning in the maintenance of trauma-centered identity. Such an assumption predicts stronger maintenance of a trauma related self-concept in independent cultures as the social role of trauma survivor/victim aligns with independent cultural expectations of the self, personal identity and the individual life story, while the social role of trauma survivor/victim contradicts the interdependent cultural expectations of a communal self.

Sutherland and Bryant (2006) posit the self-defining memory and personal goal tasks are constructive approaches to understanding autobiographical memories in PTSD because traumatic experiences often alter one's self construct (Conway, 2005). Study 1c, therefore, has used self-defining memories and personal goals to explore cultural differences in the relationship between trauma-centered self-construct and psychological adjustment. 


\subsection{Hypotheses}

In light of the above, it is hypothesized that:

$1 /$ the cultural distinction between autonomous versus relatedness orientation will be evident in everyday memories but not in trauma memories. Specifically, while the Australian group will foreground autonomy and the Asian group will foreground relatedness in the autobiographical memories of everyday events, the autobiographical memories of trauma for both Australians and Asians will be predominately autonomous (Study 1a),

$2 /$ cultural differences in the quality of the retrieved memories will depend on the theme of the memory with autonomously-themed memories and trauma-themed memories more elaborate for Australian participants and relatedness-themed memories more elaborate for Asian participants (Study 1b), and $3 /$ disrupted adjustment to the trauma measured by symptom self-report will be related to stronger personal goals and self-definition centered on trauma for Australians but not for Asians (Study 1c).

\subsection{Method}

\subsubsection{Participants}

All participants were recruited from psychology courses at the Australian National University and received extra credit for their participation. The Australian sample (10 males, 16 females) ranged in age from 17 to 42 years $(M=22.46, S D=$ 6.40) and had lived in Australia for 14 to 42 years $(M=21.60, S D=6.39)$. This entire sample reported that they spoke English at home and the religious affiliation distribution was $73.08 \%$ no religious affiliation, $23.08 \%$ Christian and $3.85 \%$ Mormon.

The Asian sample ( 8 males, 16 females) was aged 18 to 25 years $(M=20.58$, $S D=1.53)$ and had lived in Australia for 1 month to 10 years $(M=2.20, S D=2.12)$. The ethnic distribution was $54.17 \%$ Chinese, $12.5 \%$ Singaporeans, $8.33 \%$ Japanese, 8.33\% Taiwanese, $8.33 \%$ Korean, $4.17 \%$ Indian, and $4.17 \%$ Bhutanese. Language spoken at home distribution was $25.00 \%$ Chinese, $20.83 \%$ Cantonese, $12.50 \%$ Mandarin, 12.50\% English, 8.33\% Japanese, 8.33\% Korean, 4.17\% Bengali, 4.17\% 
Taiwanese and 4.17\% Dzongkha, and religious distribution was $41.67 \%$ no religious affiliation, $41.67 \%$ Christian, $8.33 \%$ Islamic and $8.33 \%$ Buddhist.

\subsubsection{Measures.}

\section{Depression.}

Given there is a high comorbidity between depression and PTSD (Brewin \& Holmes, 2003) and depression has been found to impact on autobiographical memory retrieval (e.g., Williams \& Scott, 1988, found that depressed patients retrieved less specific autobiographical memories than did non-depressed patients), depression will be controlled for throughout the research. Depression will be measured using the Hopkins Symptom Checklist (HSCL-25; Derogatis, Lipman, Rickels, \& Cori, 1974). The HSCL-25 has two parts. Part I has 10 items that measure anxiety symptoms and Part II has 15 items that measure depression symptoms. Participants are required to indicate how much each symptom bothered or distressed them in the past week, including today from 1 (not at all) to 4 (extremely). The depression score is the average of the 15 depression items. The HSCL-25 depression score has been consistently shown in several populations to be correlated with major depression as defined by the DSM-IV (1994), the HSCL-25 has adequate psychometric properties (Derogatis et al., 1974) and is regularly used in cross-culture research (e.g., Mouanoutoua \& Brown, 1995).

\section{Demographics.}

Participants were asked to disclose their age, gender, and length of time in Australia, country of birth, language spoken at home and religious affiliation. Following this, participants were asked to rate on a 10-point Likert-type scale from 1 (not at all) to 10 (extremely) how hard they found the study.

\section{Autobiographical memory for traumatic event.}

Participants were asked to "Please think about a significant, emotionally, traumatic event that you have personally experienced. Please write about this event for 5 minutes in as much detail as you can. All your writing will be completely confidential. As you write do not worry about punctuation or grammar, just really let go and write as much as you can”. Following the narrative task participants were asked to report on 10-point Likert-type scales, from 1 (not at all) to 10 (extremely), how traumatic the event was and how much of an impact it had on the participant. Participants were then asked to indicate the year in which the trauma occurred. 


\section{Autobiographical memory for everyday event.}

Participants were asked to "Think about one event you did on the weekend. Please write about this event for 5 minutes in as much detail as you can. All your writing will be completely confidential. As you write do not worry about punctuation or grammar, just really let go and write as much as you can".

\section{Self-defining memory task.}

Singer and Salovey's (1993) method to elicit self-defining memories was used. Participants were instructed that, "a self-defining memory is a memory from your life that you remember very clearly, is important to you and leads to strong positive or negative feelings. It is the kind of memory that helps you to understand who you are, and conveys powerfully how you have come to be the person you currently are." Participants were given eight minutes to write five self-defining memories.

\section{Personal goals.}

Following others researchers' (e.g., Emmons, 1986; Sutherland \& Bryant, 2006) measure of personal strivings participants were instructed "What are your most important goals at this point? Please write down as many as you can in the two minutes provided".

\section{Adjustment to the trauma event.}

Disrupted adjustment to the trauma event disclosed in the trauma narrative task was assessed using the Impact of Event Scale-Revised (IES-R; Weiss \& Marmar, 1997), a prominently selected measure to assess trauma symptomatology. The IES-R parallels the DSM-IV (1994) criteria for PTSD and is hence comprised of avoidance, intrusions and hyperarousal scales. The IES-R is a 22-item self-report measure designed to assess current subjective distress for any specific life event. Respondents are asked to rate each item on a scale from 0 (not at all) to 4 (extremely) according to the past seven days. Participants receive a score for each scale and a total score. The IES-R has adequate psychometric properties (Briere, 1997; Weiss \& Marmar, 1997) and has been used in previous cross-cultural research (e.g., Ho, 1999; Wu \& Chan, 2004).

\subsubsection{Procedure}

Data for Study 1 was collected in the same experimental session. Each experimental session took 60 minutes. Following written informed consent 
procedures, participants were asked to complete the data booklet. Participants provided personal goals, self-defining memories, a trauma memory narrative, and an everyday memory narrative (the two narrative tasks were counterbalanced). Participants were then asked to complete the trauma self-report questions assessing severity, impact and time of the trauma reported in the narrative task, the Impact of Event Scale-Revised, the HSCL-25 and demographics. Participants were instructed by the researcher to stop after timed tasks. Half of the participants from each cultural group completed the questionnaire in a group-setting comprised of between 5 and 10 participants from both cultural groups. The other half of each cultural group completed the tasks individually. This was to control for the possible impact of task setting (group vs. individual administration) on the self and subsequently on the relationship between self and autobiographical remembering. If participants completed the tasks prior to the designated time they were requested to wait until the researcher instructed them to move on to the next task. The same Caucasian researcher administered all experimental sessions.

\subsubsection{Scoring of Narratives}

A coding scheme was developed based on autobiographical memory variables that have been tested in various previous studies (Han et al., 1998; Wang, 2001; Wang \& Conway, 2004; Wang \& Leichtman, 2000; Wang et al., 1998).

\section{Theme.}

Two raters coded each memory into one of the following categories that reflected the content themes. (1) Autonomy theme was associated with objects or events in the environment and not particularly related to other people (e.g., academic achievement, sporting endeavors, etc.); and (2) relatedness theme was about collective activities of the family, workplace, community or other social groups.

\section{Autonomous orientation.}

This variable was a measure of participants' tendency to emphasize autonomy in their memories. The raters counted the number of occurrences of the following instances. The scores of these six instances were combined to produce a single score of autonomous orientation for each memory: (1) reference to personal needs, desires or preferences; (2) reference to personal dislikes or avoidance; (3) reference to personal evaluations, judgments, or opinions regarding other people, objects, or events; (4) reference to retaining control over one's own actions and resisting group 
or social pressure; (5) reference to personal achievement or competency; and (6) the number of instances that involved just the individual.

\section{Other/self ratio.}

The rater counted the number of times participants mentioned themselves and other people in their memories, respectively. An "other/self ratio" was then calculated for each memory.

\section{Interaction scenario.}

The number of instances that involved social interactions or group activities were counted and totaled for each memory.

\section{Reflective comment.}

The raters counted the number of comments participants made that entailed their reflections on mores or world-views deriving from their memory event.

\section{Memory specificity.}

Raters coded the memories as specific if the event happened at a particular point in time or memories were coded general if the event occurred regularly or on multiple occasions (Pillemer, 1998).

\subsubsection{Scoring of Self-Defining Memories}

Theme, autonomous orientation, other/self ratio and interaction scenario was scored in the self-defining memories as outlined for the narratives. The score of each of these variables was totaled across the number of memories provided. To control for fluency (as not all participants retrieved five self-defining memories) ratios were developed by dividing total scores by the number of memories reported.

\section{Elaboration.}

Following Reviere and Bakeman's (2001) elaboration was scored as the number of unique, non-redundant units of information (e.g., person, place, feeling, descriptor, personal evaluation, dialogue, etc.). For each participant, the average number of units of elaboration for autonomous-themed memories, relatedness-themed memories, trauma-themed memories and non-trauma-themed memories was calculated. Using elaboration for autonomous-themed memories as an example, the total number of elaboration units for autonomous-themed memories was calculated and divided by the number of autonomous-themed memories retrieved to provide the average number of units of elaboration for autonomous-themed memories. This was 
done likewise for relatedness-themed, trauma-themed and non-trauma-themed memories.

\section{Specificity.}

Specificity was coded as outlined for narratives. For each participant, the average number of specific autonomous-themed memories and specific relatednessthemed memories was calculated. Using autonomous-themed memories as an example, the total number of specific autonomous-themed memories was calculated and divided by the number of autonomous-themed memories retrieved to provide the average number of specific autonomous-themed memories. This was done likewise for relatedness-themed memories.

\section{Trauma-theme.}

In order to control for possible differences in the number of goals and memories provided, as not all participants provided the requested number of goals and memories, adopting Jobson and O'Kearney's (2006) approach, ratios were developed for the following variables. Each self-defining memory was coded as trauma-themed if it was directly and clearly related to the trauma-theme of the trauma narrative in Study 1a. The total number of trauma-themed self-defining memories was tallied for each participant, and then divided by the number of memories retrieved, to provide a trauma-theme ratio.

Each goal was coded as trauma-themed if the goal was directly and clearly related to trauma or survival (e.g., I want to survive, get over the trauma, etc.). The total number of trauma-themed goals was tallied for each participant and then divided by the number of goals provided to provide a trauma-theme ratio.

\subsubsection{Reliability}

Study Ia.

A second independent Caucasian rater blind to study hypotheses coded $20 \%$ of responses of the current data for theme, autonomous orientation, interaction scenario, reflective comment, and specificity. Raters had $80 \%$ agreement on theme and $78 \%$ agreement on specificity. The mean kappa coefficient of reliability for each condition was .65 for autonomous orientation, .85 for interaction scenario and 1.00 for reflective comment. 


\section{Study 1 b.}

One rater coded all the memories and a second independent rater from a Chinese cultural background blind to study hypotheses coded $20 \%$ of responses. A Chinese rater was chosen to address a concern of Study 1a, regarding having two Caucasian raters to code memories. Raters were blind to the cultural group of participants. Discrepancies between raters were resolved through discussion. The mean kappa coefficient of reliability for each condition was .83 for theme, .84 for autonomous orientation, .96 for elaboration, .79 for interaction scenarios and .75 for specificity.

\section{Study $1 c$.}

Given that coding of trauma-theme for the self-defining memories was objective (i.e. the self-defining memory had to be directly and clearly associated with the trauma narrative retrieved in Study 1a), reliability of self-defining memory trauma-theme was not necessary. Additionally, as reported in the results section, the goals measure was unable to be used as a measure of trauma-theme and hence, reliability of goal trauma-theme was not needed.

\subsection{Results}

Throughout this thesis, before hypothesis testing, the data were screened to evaluate for outliers $(M \pm 3 S D)$, normality, linearity and homoscedasticity. Unless otherwise specified data was determined appropriate for all analyses conducted. The mean substitution method accounted for missing variables (Roth \& Switzer, 1995). Data were analyzed using SPSS (version 15) and the probability of a Type I error was maintained at .05 for all analyses.

\subsubsection{Participant Characteristics}

The two groups did not differ in terms of age, $t(48)=1.40, p=.17$, or in gender distribution, $\chi^{2}(1, N=50)=.14, p=.71$. Asians had lived in Australia for significantly less time than Australians, $t(48)=14.16, p<.01$, but reported that they found the tasks no more difficult than Australians $(M=5.33, S D=2.35 ; M=4.96, S D$ $=2.18$, respectively), $t(48)=.58, p=.57$. The groups did not differ in terms of depression, $t(48)=.45, p=.51(M=1.76, S D=.60 ; M=1.86, S D=.43$, Australians 


\subsection{Study 1a}

The hypothesis tested in Study 1a is that the cultural distinction between autonomous versus relatedness orientation will be evident in everyday memories but not in trauma memories. Specifically, while the Australian group will foreground autonomy and the Asian group will foreground relatedness in the autobiographical memories of everyday events, the autobiographical memories of trauma for both Australians and Asians will be predominately autonomous.

\subsubsection{Results}

\section{Preliminary analysis for narrative task.}

There was no significant difference between Australians and Asians reports of how traumatic they found the trauma event $(M=9.27, S D=1.08 ; M=9.04, S D=$ 1.16 , respectively), $t(48)=.72, p=.48$, event impact $(M=8.96, S D=1.25 ; M=8.75$, $S D=1.62), t(48)=.52, p=.61$, or time of event, $(M=5.81$ years, $S D=4.23$ years; $M=6.46$ years, $S D=5.12$ years, respectively), $t(48)=.26, p=.63$. The trauma narratives were classified into the following trauma type categories; DSM-IV (1994) criterion A trauma type (i.e., witness death, environmental disaster, serious physical injury, sexual assault, motor vehicle accident) (Australian $n=8$; Asian $n=5$ ); academic or work failing (Australian $n=1$; Asian $n=5$ ); family breakup/difficulties/death (Australian $n=6$; Asian $n=6$ ), and peer rejection/bullying/friendship break-up (Australian $n=11$; Asian $n=8$ ). The two groups did not differ in terms of trauma type distribution, $\chi^{2}(3, N=50)=3.76, p=$ .29 .

A $2 \times 2$ way ANOVA with culture (Asian vs. Australian) as a between subject factor and memory (everyday vs. trauma) as a within subject factor with number of words as the dependent measure, revealed no differences in fluency of everyday $(M=$ $108.12, S D=29.00)$ versus trauma $(M=109.18, S D=30.22)$ memories overall, $F(1$, $48)=.13, p=.72$. However, overall Asians $(M=96.44, S D=21.83)$ had significantly shorter narratives than Australian students $(M=119.92, S D=31.25), F(1,48)=$ $11.38, p<.01$, and this cultural difference was not moderated by memory type, $F$ (1, 48) $=1.51, p=.23$. 


\section{Comparative analysis of narratives.}

Theme. To examine cultural differences in the memory theme of the everyday memories and trauma memories, a chi-square with culture (Asian; Australian) and theme (autonomous; relatedness) for the memories was used. For the everyday memory the two groups did differ in terms of memory theme distribution, $\chi^{2}(1, N=$ $50)=4.99, p<.05$. Australians were more likely to provide autonomous themed everyday memories (46.15\%) compared with their Asians counterparts (16.67\%). Asians were more likely to provide relatedness themed everyday memories (83.33\%) compared with their Australian student counterparts (53.85\%). For the trauma memory, the two groups did not differ in terms of memory theme distribution, $\chi^{2}(1, N$ $=50)=.30, p>.05$. Asians were as likely to provide autonomous themed trauma memories (50.00\%) as their Australian counterparts (42.31\%). Australians were as likely to provide relatedness themed trauma memories (57.69\%) compared with their Asian student counterparts (50.00\%).

Autonomous orientation. To examine culturally dependent differences in the other narratives measures $2 \times 2$ way ANOVAs with culture (Asian vs. Australian) as a between subject factor and memory (everyday vs. trauma) as a within subject factor was used.

Autonomous orientation was significantly greater in the trauma memories than in the everyday memories overall, $F(1,48)=5.61, p=.02$. There was no significant difference between Australians and Asians in autonomous orientation overall, $F$ (1, $48)=3.02, p=.09$. Additionally, the interaction between culture and memory was significant, $F(1,48)=4.08, p=.049$. Australians had significantly greater autonomous orientation in their everyday memories than did Asians $t(48)=3.04, p<$ $.01, C 1.95=.97,4.73, d=.87$, but did not differ from Asians in the autonomous orientation of their trauma memories, $t(48)=.13, p=.90, C I .95=-2.31,2.63, d=.04$. Further, Australians did not differ in autonomous orientation in their trauma and everyday memories, $t(25)=.24, p=.82, C I .95=-1.79,2.26$, but Asians had significantly greater autonomous orientation in their trauma memories than their everyday memories, $t(23)=3.31, p<.01, C I .95=1.09,4.74$ (Figure 5.1) . 


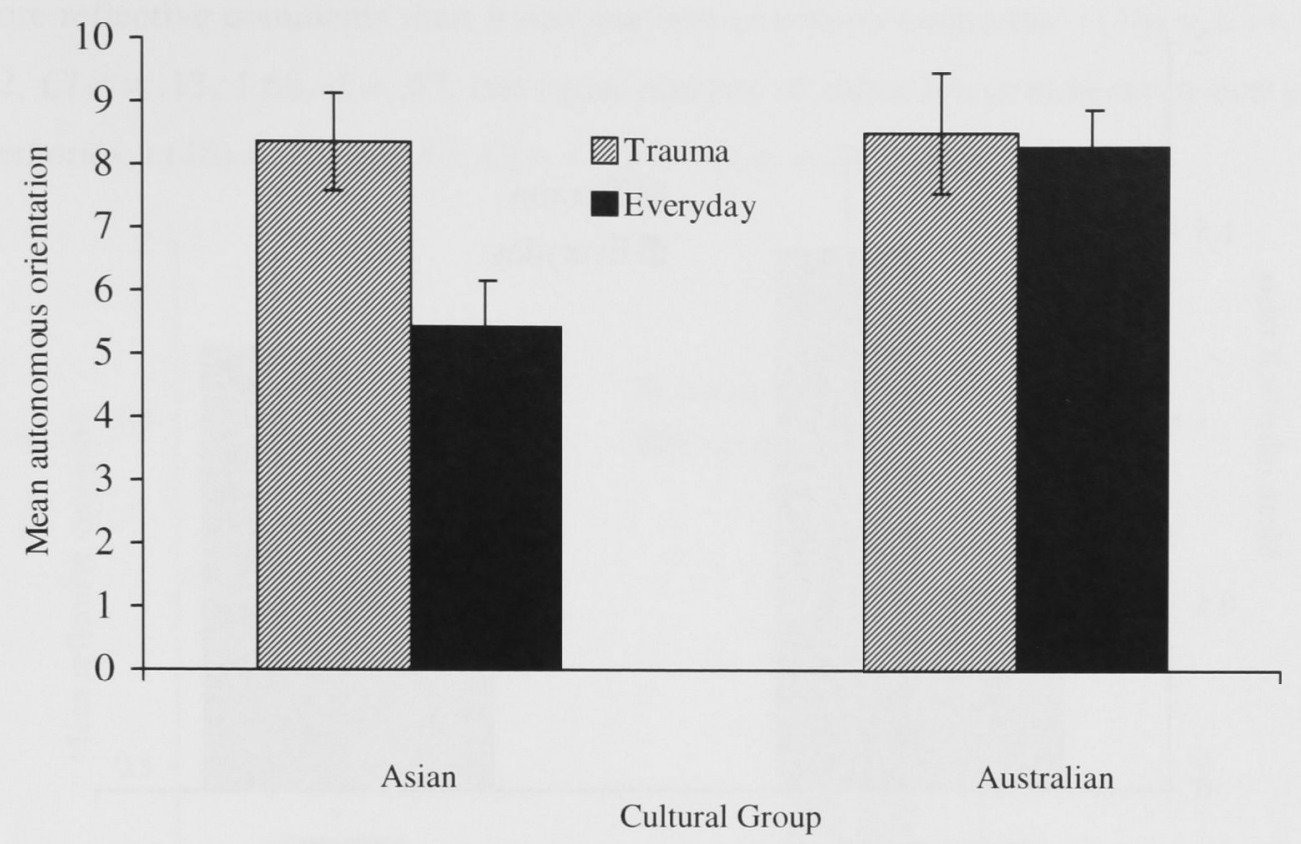

Figure 5.1 Mean autonomous orientation of everyday and trauma memories for Asian international students and Australian domestic students. Error bars indicate \pm 1 SE of the mean.

Other/self ratio. The other/self ratio was significantly greater in the everyday memory than the trauma memory overall, $F(1,48)=17.48, p<.01$. However, there was no significant difference between Asians and Australians in other/self ratio overall, $F(1,48)=.01, p=.93$. There was a significant interaction between culture and narrative type for other/self ratio, $F(1,48)=4.16, p=.047$, with Asians providing a significantly higher other/self ratio for everyday memories than trauma memories, $t(48)=4.04, p<.01, C I .95=.44,1.37$, whereas Australians provided equal other/self ratios for both trauma and everyday memories, $t(48)=1.66, p=.25, C l .95=$ - .70, .08. However, the Asian and Australian groups did not differ in other/self ratio in either the everyday, $t(48)=.74, p=.47, C I .95=-1.01, .47, d=.21$, or trauma memory, $t(48)=1.17, p=.25, C I .95=-.23, .88, d=.34$ (Figure 5.2). 


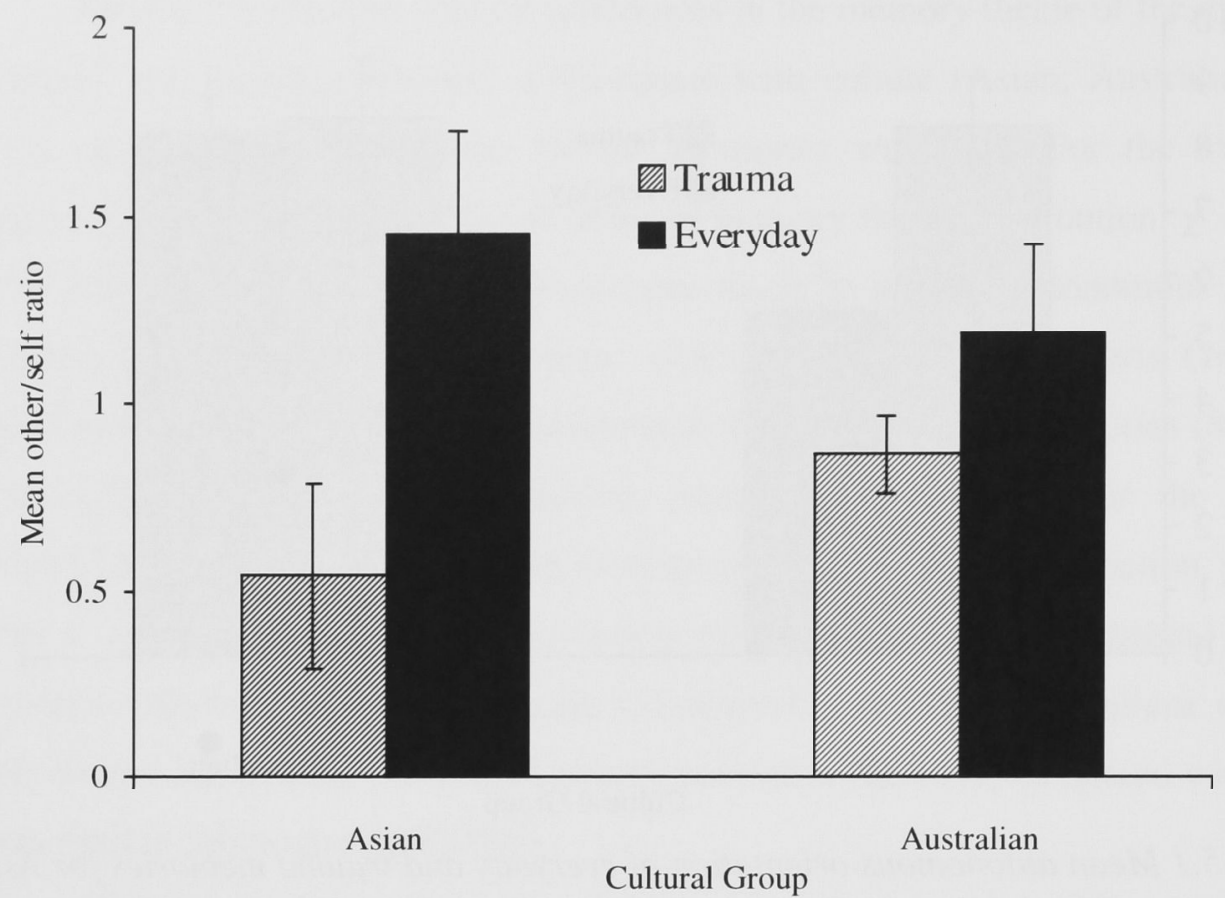

Figure 5.2 Mean other/self ratio of everyday and trauma memories for Asian international students and Australian domestic students. Error bars indicate \pm 1 SE of the mean.

Interaction scenario. The number of social interactions was significantly greater in the everyday memories $(M=3.50, S D=1.66)$ than trauma memories $(M=$ 2.36, $S D=1.66)$ overall, $F(1,48)=9.38, p<.01$. Further, Asians $(M=3.40, S D=$ 2.39) had significantly more social interactions than did Australians $(M=2.50, S D=$ 1.70 ) in their memories overall, $F(1,48)=4.54, p=.04$. There was no significant interaction between culture and narrative type for interaction scenario, $F(1,48)=.36$, $p=.55$.

Reflective comment. Reflective comments were significantly greater for the trauma memories than the everyday memories overall, $F(1,48)=18.16, p<.01$. There was no significant difference between Asians and Australians in reflective comments, $F(1,48)=3.59, p=.06$. There was a significant interaction between culture and narrative type for reflective comments, $F(1,48)=8.75, p<.01$, with Australians providing significantly more reflective comments in the trauma memory than in the everyday memory, $t(48)=4.17, p<.01, C I .95=.45,1.33$, whereas Asians provided equal number of reflective comments for both trauma and everyday 
memories, $t(48)=1.45, p=.16, C I .95=-.07, .41$. Australians provided significantly more reflective comments than Asian students in trauma memories, $t(48)=2.34, p=$ $.02, C I .95=.13,1.68, d=.67$, but equal number of reflective comments in everyday memories, $t(48)=.76, p=.45, C I .95=-.24, .53, d=.21$ (Figure 5.3).

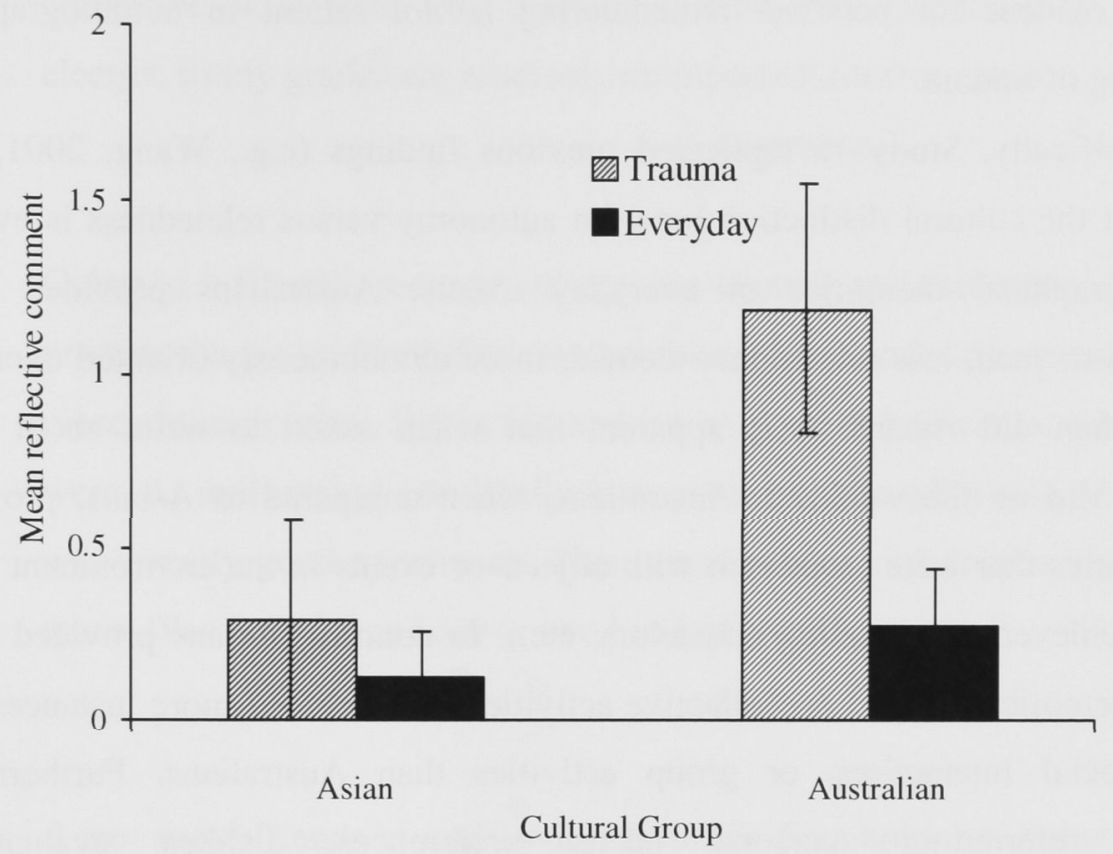

Figure 5.3 Mean reflective comment of everyday and trauma memories for Asian international students and Australian domestic students. Error bars indicate \pm 1 SE of the mean.

Memory specificity. To examine culturally dependent differences in the memory specificity of the narratives, a chi-square with culture (Asian; Australian) and specificity (specific; general) for each narrative-type was used. The two groups differed in terms of trauma memory specificity distribution, $\chi^{2}(1, N=50)=9.74, p<$ .01. Compared with their Asian counterparts (37.50\%), Australians (80.77\%) tended to provide more specific trauma memories. Unexpectedly, the two groups did not differ in terms of everyday memory specificity distribution, $\chi^{2}(1, N=50)=.01, p=$ 92. That is, both Asians $(87.5 \%)$ and Australians $(88.46 \%)$ provided highly specific everyday memories. These results were evident even when depression was controlled for. 


\subsubsection{Discussion}

The results confirm the expectations derived from the conceptual framework that because traumatic events by their nature activate an autonomous goal hierarchy or working self then the cultural distinction between autonomy versus relatedness orientation evident for personal remembering is not salient in autobiographical remembering of trauma.

Specifically, Study 1a replicated previous findings (e.g., Wang, 2001) that indicate that the cultural distinction between autonomy versus relatedness is evident in autobiographical memories of everyday events; Australians provided more autonomous-themed, less relatedness-themed, more autonomously oriented everyday memories than did Asians. It is apparent that when asked to write about what participants did on the weekend, Australians, when compared to Asians, provided more memories that were associated with objects or events in the environment (e.g., academic achievement, sporting endeavors, etc.). In contrast, Asians provided more everyday memories focused on collective activities and included more instances that involved social interactions or group activities than Australians. Furthermore, Australians referred to personal needs, preferences, dislikes, evaluations, achievements, personal control and to instances that involved just the individual more than Asians. These findings are evident in the following selected everyday memories that resemble the typical response of each cultural group.

I worked over the weekend at the World Superbike Championships on Phillip Island in Victoria. I was working with an Italian crew combined with Australians on the world television coverage. I was the vision switcher, in charge of cutting cameras, visual effects etc. but also had to interpret the Italian directors dodgy English so that the 14 cameramen knew what was going on and what to cover. It was challenging and I thoroughly enjoyed the role I was playing. I was able to catch up with old work colleagues and also observe how another culture operates in a television sense...

- Australian

On the weekend I wrote my first psych[ology] lab[oratory] report which is due today. I found it very difficult as no outline was given as to the extent of the statistical analysis we were supposed to do. I also had to work for 6 hours on 
Saturday and Sunday so had limited time to write the report. Excel on my computer did not have the necessary tools to complete the descriptions. As I had done psych[ology] in college I assumed that the report would cover the same kind of structure but the results sections in particular was very difficult. I completed it at $3 \mathrm{am}$ this morning. Fortunately, psych[ology] is only an elective so my grades are relatively unimportant for this subject.

\section{- Australian}

Going to a friend's room at college, we chatted and then went downstairs to practice the piano. She called another friend down and we just played different pieces to each other. It was fun and it was good to know how talented people were. At uni[versity] you hardly ever get to see the other side of people so it was a really good experience. Then we ate some junk food and chatted a bit more. Was only for an hour or two but had great fun.

\section{- Asian}

I met some friends at uni[versity] from the foundation year that I studied in the University of New South Wales. We were 5 of us and we decided to eat and cook dinner at one friend's house. Everyone except one (that is my best friend) wanted to change their mind and eat KFC after a few minutes. But I knew that my best friend didn't want KFC. Then because I am the one who had to drive I drove to the supermarket and we didn't have KFC. After that dinner we played computer games and the others had to wait for me to finish the game and drive them home.

There was, however, no cultural distinction in how Australians or Asians remembered a personal traumatic event in terms of the memory's theme, degree of autonomous content or proportion of references to self or others. That is, in the following examples it is clear that when asked to write about a personal trauma event, Australians and Asians provide similarly themed trauma memories in terms of autonomy and relatedness. Furthermore, Australians remained as autonomously oriented and referred to themselves and others equivalently in their trauma and everyday memories. However, it is apparent that Asians refer to personal needs, 
desires, dislikes, evaluations, opinions, control, achievements or failures, and to instances that involved just the individual more in the trauma memory than in the everyday memory; a level comparable to that of Australians. Asians also provided more egotistic trauma memories than everyday memories.

It probably doesn't sound too traumatic to others but for me, someone who has always participated in sport, enjoyed keeping fit, experiencing a knee injury changed my life. I had injured it six years earlier but had overcome any effects of surgery and continued as I always had. One night, playing basketball, my knee gave way, not only snapping the anterior cruciate ligament but shearing off a large chunk of cartilage. This meant that even though the operation was successful I still cannot run. This precludes me from running to keep fit and playing team sport.

\section{- Australian}

Not long after my parent's divorce/separation I would have been roughly 6 years old, my brother and I had a trial visit with my dad who we had seen very little of for a few months. He was staying in a caravan on the property that he and my mother had bought together and had begun building a new house. We were to stay the night. I don't remember the day clearly but I can remember feeling happy, feeling safe, enjoying myself. Then I can remember my mother arriving unexpectedly in the evening and insisting that we (my brother and I) go home with her. I can vividly remember crying.

\section{- Australian}

A traumatic event for me was that I really wanted to go to the University of Queensland as I got into B[achelor] Pharmacy degree. However, the tuition fees for International students are too high at U[niversity of] Q[ueensland] and I don't have that much money to study pharmacy for 4 years. I really hate to give up pharmacy. I don't really like Canberra when I came, I really wanted to go back to Brisbane at the beginning as I stayed there for 3 years and there were familiar places and people there. Even though it is getting better now I still don't like Canberra. 
The first time I broke up with my boyfriend. I was so emotional afterwards. I was so unhappy, sad, did nothing, feeling low the whole day. I cried, cried and cried. I locked myself in the room, didn't want to talk with anyone, even my family. I wished I was dreaming and everything will be changed...I was collapsed and so angry. This event until now still puts a sword into my heart. And after this I decided to leave Hong Kong for a while and came to Australia to start my new life, to somewhere that no one will know me.

Contrary to Wang and Conway's (2004) finding that Chinese participants more frequently contained reflective comments in their everyday memories, this first study found no cultural differences in the number of reflective comments made in everyday memories. This may be the result of Wang and Conway (2004) requesting participants to recall twenty memories from any period of their lives, while the present study gave specific instructions to retrieve an event from the weekend. It seems likely that retrieving a memory of what one did on the weekend would be less likely to generate reflective comments than that of salient life memories. What did differ, however, was that Australian students provided significantly more reflective comments than Asian students in trauma memories. For example, Australians included comments like "only recently, three years on, have I started to let it go. It's still in the background but I've learnt from it" and "I was at my lowest but with a smile on my face I knew the only way was up", whereas Asians tended not to include such comments. This may be because the trauma memory, as an autonomous memory, concurs with the Australians' autonomous life story but is disjunct from the Asians' relatedness, collective story, mores and worldviews, resulting in less opportunity and resources for reflection in Asians' narratives. The implication of this finding is interesting, as less reflective comments may result in less contemplation, contexualization and exposure to the trauma memory. Reflective comment may also allow meaning and a shifting of goals, which would aid in trauma memory integration.

Previous research (e.g., Han et al., 1998) has found that even when people are explicitly asked to recall specific events, Asians still tend to provide general memories. Wang and Conway (2004) suggest that a "cultural emphasis on autonomy 
may encourage remembering significant personal experiences with specific details...especially those of one-moment-in-time events unique to an individual" (p. 912). Hence, independent cultures provide specific memories in order to "serve an important means to differentiate the self from others, thereby re-affirming the self as an autonomous self' (Wang \& Conway, 2004, p. 912). However, the provision of specific autobiographical memories by interdependent cultures may be viewed, culturally, as dysfunctional and unnecessary in "reaffirming the self as a relational self" (p.913). In this study no cultural differences in everyday memory specificity was found. It is suggested that this be the result of very clear instructions, which asked participants to write about one event they did on the weekend. However, Australian students provided significantly more specific trauma memories than Asian students. This finding suggests that despite the content of the memories of Asians being moderated by trauma, the quality, in terms of specificity of the memory, may not be moderated by trauma. Furthermore, this finding, if robust, may have major legal (e.g., specific memories are often deemed more credible than general memories, e.g., Herlihy and Turner, 2002) and clinical implications (e.g., interdependent cultures providing general trauma memories may impact on assessment, exposure work and cognitive re-structuring where specific memories are generally thought to be required for an efficacious outcome, e.g., Ehlers \& Clark, 2000).

In sum, the results support the conceptual framework's prediction that the cultural distinction between autonomy versus relatedness orientation evident for autobiographical remembering is not salient in autobiographical remembering of trauma. Specifically, Study 1a replicated previous findings (e.g., Wang \& Conway, 2004) that indicate those from independent cultures are more likely to retrieve autobiographical memories which foreground autonomy, while those from interdependent culture are more likely to retrieve autobiographical memories which foreground relatedness. Second, the results support the conceptual framework's prediction that trauma by definition activates an autonomous goal hierarchy or working self and thus, in order to maintain an autonomous sense of self there is a universal foregrounding of autonomy in the remembering of traumatic events. In both cultural groups the theme, autonomous orientation and level of egocentricity of the trauma memory did not differ. Third, the results supported the framework's prediction that while in the autobiographical remembering of trauma for independent cultures, the working and conceptual selves are aligned for interdependent cultures the 
conceptual self gives way to the autonomous working self. This was supported by the finding that for independent culture the level of autonomous orientation was comparable in both memory types, whilst for interdependent cultures autonomous orientation was significantly greater in their trauma memories than their everyday memories. 


\subsection{Study $1 b$}

The hypothesis tested in Study $1 \mathrm{~b}$ is that cultural differences in the quality of the retrieved memories will depend on the theme of the memory with autonomouslythemed memories and trauma-themed memories more elaborate for Australian participants and relatedness-themed memories more elaborate for Asian participants.

\subsubsection{Results}

\section{Preliminary analysis.}

Australians and Asians provided an equivalent number of self-defining memories, $(M=4.77, S D=.51 ; M=4.92, S D=.28$, respectively $), t(48)=1.24, p=$ $.22, C I .95=-.39, .09$. Self-defining memories were equivalent in length (mean number of words; $M=18.05, S D=7.77 ; M=17.23, S D=7.18$, respectively), $t(48)=.39, p=$ $.70, C I .95=-3.44,5.09$.

\section{Content comparative analysis of self-defining memories.}

Content comparative analysis was undertaken using independent $t$ tests.

Australians provided a significantly higher proportion of autonomous-themed self-defining memories $(M=.61, S D=.24)$ than Asians $(M=.46, S D=.26), t(48)=$ $2.16, p=.04, C 1.95=.03, .30, d=.60$, and Asians a significantly higher proportion of relatedness-themed memories $(M=.53, S D=.23)$ than did Australians $(M=.37, S D=$ $.23), t(48)=2.40, p=.02, C I .95=.01, .30, d=.70$. Australians used more references which emphasized autonomy in their self-defining memories $(M=3.99, S D=1.88)$ than Asians $(M=1.52, S D=.59), t(48)=6.17, p<.01, C I .95=1.66,3.27, d=1.77$. Asians $(M=1.01, S D=.67)$ referred more to social or group activities in their selfdefining memories than Australians $(M=.45, S D=.24), t(48)=4.04, p<.01, C 1.95=$ $.28, .84, d=1.11$. Although the results were in the expected direction, Asians $(M=$ $.44, S D=.27)$ and Australians $(M=.30, S D=.35)$ did not differ in other/self ratio, $t$ $(48)=1.53, p=.13, C I .95=-.32, .04, d=.45$.

\section{Structure comparative analysis of self-defining memories.}

Elaboration. There was no difference in the elaboration of autonomous versus relatedness-themed memories overall, $F(1,48)=.09, p=.77$. The difference between Australians and Asians in elaboration of their self-defining memories overall was not significant, $F(1,48)=.18, p=.67$. However, there was a significant interaction between culture and theme for elaboration, $F(1,48)=12.57, p<.01$ (Figure 5.4). 
Australians provided significantly more elaboration than Asians for autonomouslythemed memories, $t(48)=2.88, p<.01, C I .95=.50,2.80, d=1.08$, but the stronger elaboration by Asians compared to Australians for relatedness-themed memories was not significant, $t(48)=1.58, p=.12, C I .95=-2.71, .33, d=.45$. Australians elaborated their autonomously-themed memories significantly more than their relatedness-themed memories, $t(25)=2.31, p=.03, C I .95=.14,2.46$, and Asians elaborated their relatedness-themed memories more than their autonomously-themed memories, $t(23)=2.71, p=.01, \quad C I .95=-2.72, \quad-.36$.

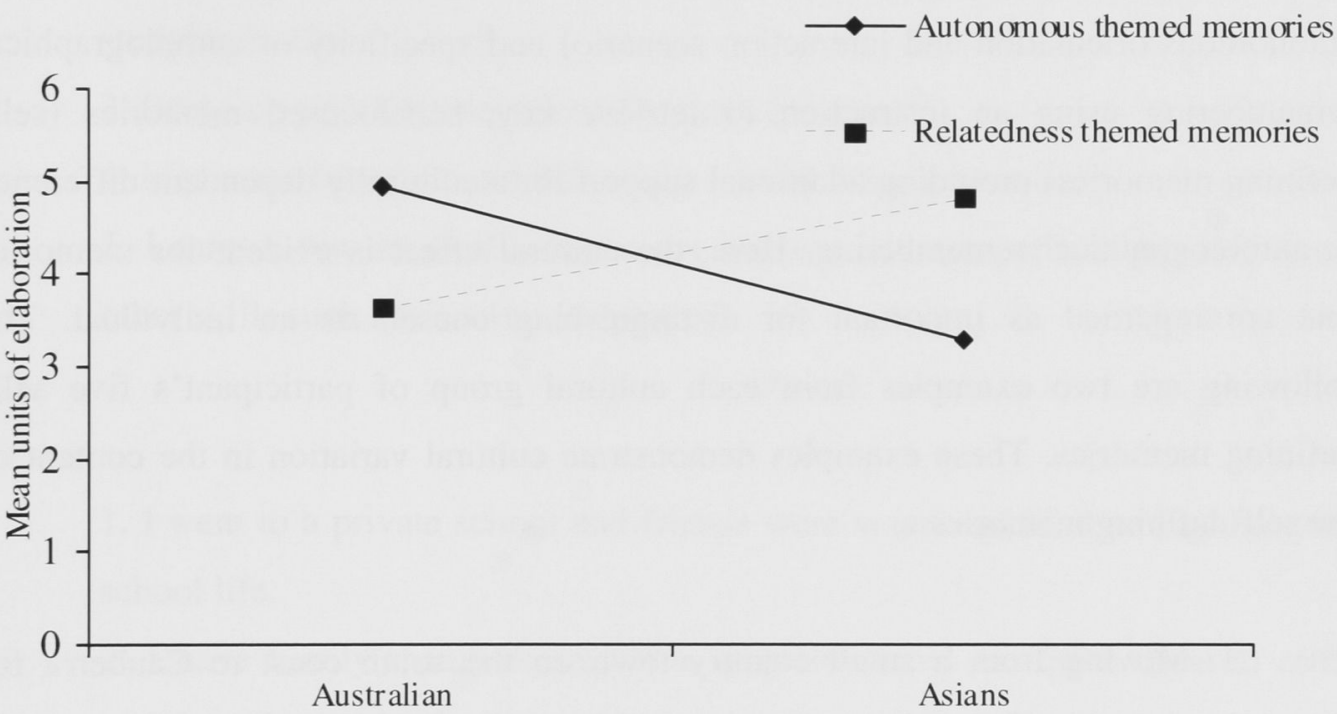

Cultural Group

Figure 5.4 Mean number of units of elaboration in memories for each group by theme of memory.

Non-trauma memories $(M=4.57, S D=1.63)$ were significantly more elaborate than trauma memories $(M=2.36, S D=2.66), F(1,48)=32.07, p<.01$. However, the Australian $(M=3.72, S D=2.33)$ and Asian $(M=3.30, S D=1.96)$ groups did not differ in terms of elaboration for these memories, $F(1,48)=.41, p=$ .52 , and unexpectedly the interaction was not significant, $F(1,48)=.60, p=.44$. 
Specificity. There was no difference in the specificity of autonomous $(M=.62$, $S D=.41)$ versus relatedness-themed $(M=.60, S D=.41)$ memories overall, $F(1,48)$ $=.05, p=.82$. There was a significant difference between Australians $(M=.73, S D=$ $.38)$ and Asians $(M=.48, S D=.40)$ in the specificity of their self-defining memories, $F(1,48)=8.69, p<.01$, this cultural difference was not moderated by the memories' theme, $F(1,48)=.03, p=.87$.

\subsubsection{Discussion}

Study $1 \mathrm{~b}$ replicated observed cultural differences in the content (i.e., theme, autonomous orientation and interaction scenario) and specificity of autobiographical remembering using an instruction to retrieve key, self-focused memories (selfdefining memories) providing additional support for a culturally dependent difference in autobiographical remembering. Here, the cultural effect is evident for memories that are regarded as important for distinguishing oneself as an individual. The following are two examples from each cultural group of participant's five selfdefining memories. These examples demonstrate cultural variation in the content of the self-defining memories.

1. Moving from a small country town on the south coast to Canberra for improved study/employment opportunities at the start of Year 11.

2. Representing the ACT at the Australian National's Cross Country Championships and placing $9^{\text {th }}$ in Year 8.

3. The first time I was admitted to Hospital at the start of year 12 following my first major overdose.

4. Being diagnosed with Borderline Personality Disorder in April of Year 12. Being informed of what this meant by the psychiatrist.

5. Waking up at Intensive Care after one week in a coma and subsequent admittance to hospital. Feeling so incredibly alone.

- Australian

1. When I was 2 years old I severed a large amount of my right finger on a folding table, sad memory.

2. My first violin performance in front of my school, age 10, happy memory. 
3. Having my first non-English dream while overseas. 7 years old. I still have some today, very happy.

4. Winning my first tennis trophy, age 14, happy.

5. Completing year 10 and 12 with almost perfect scores i.e. A's or B's happy.

- Australian

1. Grew up in Mum's city then returned to Dad's city and started primary school.

2. Was always left at home alone since parents were busy working when I was studying in China.

3. Broke up with first boyfriend for some reason.

4. Met some friends that did the most hurtful thing ever to me.

5. Learnt a few musical instruments and was always performing, also some other skills such as dancing, singing etc.

\section{- Asian}

1. I went to a private school and friends were smart and kind so I had a great school life.

2. I have family members who live long lives so I am quite close to older people and consider family members to be important.

3. I have asthma so I am quite sensitive to the environment and always have to think what could start it.

4. My mother has always been strict on me but when I have children of my own, I don't plan to be so strict.

5. I have never had a close relationship with my father so I don't know how to communicate with older men

- Asian

In terms of memory quality, the findings suggest that cultural differences in the quality of autobiographical memories depend on the theme of the memory. Specifically, the results show that elaboration of autobiographical memories may occur equally for people from interdependent cultures as for people from independent cultures and that elaborating autobiographical memories is dependent on memory content. These results are consistent with the conceptual framework's position 
regarding culturally appropriate elaboration and integration of autobiographical memories and SMS' functional perspective on autographical remembering. The results question suggestions that those from interdependent focused cultures may have "less need for elaborated, detailed autobiographical memories" (Wang, 2001, p.221) and that their autobiographical histories can be depicted as "brief, skeletal" (Conway et al., 2005, p.740). The findings indicate instead that elaborating autobiographical memories may function to enhance the dominant self-focus by enriching the network and presentation of the relatedness-self for Asians and the autonomous-self for Australians.

The hypothesis of Australians retrieving more elaborate trauma memories than Asians, as a result of the trauma memory being predominately autonomous and culturally sanctioned in independent culture, was not supported. Both cultures retrieved significantly more elaborate non-trauma memories when compared to trauma memories. This finding reflects the theoretical approaches (e.g., appraisal model and the SMS) that posit poor elaboration of the trauma autobiographical memory and further supports the notion of universal commonalities in the trauma memory found in Study 1a.

In sum, the results support the conceptual framework implication that the cultural distinction in autobiographical memory quality depends on the memories' theme. It appears that both the content and quality of autobiographical remembering function to reaffirm the culturally sanctioned self; specifically both content and structure of autobiographical memories help regulate affect by ensuring a correspondence between acts of remembering and the dominant conceptual selfknowledge as specified in SMS (Conway, 2005). 


\subsection{Study 1c}

Study 1c tested the hypothesis that disrupted adjustment to the trauma measured by symptom self-report will be related to stronger personal goals and selfdefinition centered on trauma for Australians but not for Asians.

\subsubsection{Results}

\section{Psychological adjustment.}

Asians $(M=27.54, S D=15.91)$ reported significantly higher on the IES-R than Australians $(M=12.15, S D=12.46), t(48)=3.82, p<.01$. Asians also reported significantly higher on the subscales; avoidance $(M=10.96, S D=6.54$; versus $M=$ $5.15, S D=5.46$ for Australians $), t(48)=3.42, p<.01$, intrusions $(M=12.17, S D=$ 8.08; versus $M=5.31, S D=5.31$ for Australians), $t(48)=3.57, p<.01$, and hyperarousal $(M=4.42, S D=4.06$; versus $M=1.69, S D=2.85$ for Australians $), t$ $(48)=2.76, p<.01$.

\section{Trauma comparative analysis.}

The trauma comparative analysis utilized an independent $t$ test followed up by two bivariate correlation matrixes; the relationships between trauma-themed memories and psychological adjustment (IES-R) were examined firstly for the Australian group and then the Asian group. The two cultural group's correlation coefficients were then compared.

Australians $(M=.16, S D=.17)$ did not differ from Asians $(M=.10, S D=.10)$ in terms of trauma ratio for self-defining memories, $t(48)=1.54, p=.13, C I .95=-.02$, $.14, d=.43$. However, there were moderate to strong positive correlations between the trauma ratio for self-defining memories and scores on the IES-R for the Australians (IES-R total, $r=.51$, intrusions, $r=.54$, avoidance, $r=.51$, hyperarousal $r$ $=.27$ ). All these correlations for the Asians were negative and, except for hyperarousal, weak (IES-R total, $r=-.12$, intrusions, $r=-.03$, avoidance, $r=-.05$, and hyperarousal, $r=-.34)$. The correlations differed significantly between Asians and Australians (IES-R, $Z_{\text {diff }}=2.28, p=.02$, intrusions, $Z_{\text {diff }}=2.09, p=.04$, avoidance, $Z_{\text {diff }}$ $=2.00, p=.04$, hyperarousal, $\left.Z_{\text {diff }}=2.08, p=.04\right)$.

While Australians and Asians provided an equivalent number of personal goals, $(M=4.19, S D=1.60 ; M=4.00, S D=1.89$ respectively $), t(48)=.39, p=.71$, $C I .95=-.78,1.14, d=.11$, the personal goals measure could not be used as a measure 
of trauma's impact on personal identity as not one participant from the Asian group provided a trauma-themed personal goal. Only two Australian students provided trauma-themed goals and hence, both participants had to be excluded as outliers.

\subsubsection{Discussion}

This study found that the impact of trauma on change in self-definition and personal identity is culturally specified. In particular, while the study replicated earlier findings (e.g., Sutherland \& Bryant, 2006) that stronger self-definition centered on trauma was positively related to severity of posttraumatic symptoms it was found that this was the case for Australians but not for Asians. For this latter group, there was no relationship between disrupted adjustment and trauma-centered self-definition or in the case of heightened arousal a relationship between stronger trauma-centered selfdefinition and lesser degree of hyperarousal.

This finding is important firstly for theoretical reasons. The SMS (Conway, 2005) and centrality of trauma approach (Berntsen and Rubin, 2006, 2007) propose that following trauma alterations in self-definition and personal identity to center on trauma, driven by a need for self-consistency and self-coherence, is related to poorer psychological adjustment. However, the finding suggests that these approaches may be limited in terms of the impact of cultural distinction in self on the degree to which trauma becomes a central component to personal identity and self-definition. These approaches seem to have stopped short of considering and making explicit the impact of cultural variability in self-consistency needs (Suh, 2000). Independent cultures expect and require self-consistency and coherence across time and context. The individual rather than the situation is the anchor of thoughts, behaviors and emotion. To clarify this, take the following as an example. Person $\mathrm{X}$, from an independent culture, is defined (in terms of self and identity) by characteristics such as kind, honest, trustworthy and so forth, and is (and is expected to be) these characteristics across time and context. However, if $\mathrm{X}$ experiences a traumatic event, an adopted characteristic may be trauma victim/survivor. This characteristic (along with trauma associated characteristics such as incompetency, afraid, etc.) is added to X's list, will transcend time and context and become a defining personal characteristic. Trauma identity is even likely to become central to personal identity resulting in poorer psychological adjustment (Berntsen \& Rubin, 2007). In contrast, person Y from an interdependent culture is (in terms of self and identity) a mother, sister, student and so 
forth, and different characteristics accompany such social roles. Hence, the focus is on the social context rather than the personal qualities of $\mathrm{Y}$. Y is much more flexible and tolerant of differences in the self in these different social contexts. Hence, when Y endures a trauma experience, trauma victim/survivor is adopted as another social role and hence is restricted, alongside trauma associated characteristics (i.e., incompetent, afraid) to context and time and will tend not to define the whole self nor alter other social roles that do contribute to self-definition (i.e., Y is still a mother, sister, student, etc.). Hence, the social role of trauma victim/survivor is not likely to distinguish between those with adaptive adjustment from those with disrupted psychological adjustment.

In addition, the results accord that the view that the social role of trauma victim/survivor may be a culturally sanctioned role in independent cultures given that it aligns with cultural expectations of the self, personal identity and the individual life story. However, in interdependent cultures the social role of trauma survivor/victim may be viewed as abnormal and culturally inappropriate, as it contradicts cultural expectations of a communal self.

To extend external validity and clinical inferences, a second study examining self-definition and more general personal identity in trauma survivors from the general community with and without PTSD, from interdependent and independent cultures is needed. If the findings are robust the theoretical and clinical implications are significant. In particular, the clinical cognitive models of PTSD which propose that self-change towards a trauma-centered self predicts poor post trauma prognosis and contributes to symptom maintenance may not be applicable across cultures. In this study, self-change was associated with trauma adjustment for participants from an independent orientated culture (Australians) but not for those from interdependent cultures (Asians). These findings suggest these models and their theoretical assumptions may need to consider cultural factors in the nature of the conceptual self in any theoretical reformulation. Clinically, they would question the relevance of the focus on the self-referential thinking that is an integral part of assessment and treatment in cognitive therapy for post-traumatic distress for example, it would suggest a lesser role for cognitive reframing of self-schema for those from Asian cultures and suggest that given interdependent cultures do suffer from PTSD, research needs to explore factors, other than self-definition, that may be involved in the development and maintenance of PTSD. 
The study failed to show cultural differences in the theme of personal goals. It is believed that this could be the result of the instructions of the task. Participants were asked to provide their most important goals at this point in time. Given participants were university students the majority of goals were related to academic success (e.g., to get an HD in my PSCY1001 essay, to do well in my degree). Hence, very few participants gave trauma-centered goals. In a future study it would be beneficial to ask this of a general population sample and word the task differently so it captures personal life goals.

In sum, the study replicated previous findings suggesting that trauma-centered self-definition was related to poorer psychological adjustment. However, this finding appears culturally determined as this was found to be the case for independent culture but not interdependent culture. There was no relationship between trauma-centered identity and psychological adjustment. 


\subsection{General Discussion}

The results of Study 1 confirm the hypotheses derived from the conceptual framework. First, the findings supported the prediction that because traumatic events by their nature activate an autonomous goal hierarchy or working self then the cultural distinction between autonomy versus relatedness orientation evident for personal remembering seems not to be salient in autobiographical remembering of trauma. Specifically, how a person remembers a traumatic event seems to be predominantly guided by autonomous goals regardless of the dominant goal orientation of his or her culture.

Second, the findings suggest that cultural differences in the quality of autobiographical memories depend on the theme of the memory. Specifically, the results show that elaboration of autobiographical memories may occur equally for people from interdependent cultures as for people from independent cultures and that elaborating autobiographical memories is dependent on memory content. These results are consistent with the SMS' functional perspective on autographical remembering. The findings indicate that elaborating autobiographical memories may function to enhance the dominant self-focus by enriching the network and presentation of the relatedness-self for Asians and the autonomous-self for Australians. The hypothesis of cultural differences in the elaboration of trauma memories was not supported. Both cultures retrieved significantly more elaborate non-trauma memories when compared to trauma memories reflecting the theoretical approaches (e.g., appraisal model and the SMS) that posit poor elaboration of the trauma autobiographical memory. This further supports the notion of cultural commonalities in the trauma memory.

Third, this study found that the impact of trauma on change in self-definition and personal identity is culturally specified. In particular, while the study replicated earlier findings (e.g., Sutherland \& Bryant, 2006) that stronger self-definition centered on trauma was positively related to severity of posttraumatic symptoms it was found that this was the case for Australians but not for Asians. For this latter group, there was no relationship between disrupted adjustment and trauma-centered self-definition. This finding is important for theoretical reasons as the findings suggest the SMS (Conway, 2005) and centrality of trauma approach (Berntsen \& Rubin, 2006, 2007) may be need to consider cultural differences in self-consistency needs and the social 
acceptance of the role of trauma survivor/victim. If these findings are robust the clinical implications may be significant. In particular, they would question the relevance of the focus on the self-referential thinking that is an integral part of assessment and treatment in cognitive therapy for post-traumatic distress.

The limitations of this study are acknowledged. First, the size and nature (university students) of the sample limits the clinical interferences from these findings. Second, as in any study exploring the impact of culture on certain variables, language and task understanding must be considered. The findings of no cultural differences in (a) the self-report of task difficulty, and (b) university standard English language competency, was taken collectively to suggest that there were no major cultural differences in task understanding and responding. Additionally, it is not clear what impact the fact that the Asians were undertaking a task in an intercultural context while for the Australians the task was intracultural, may have had on the findings. An individualistic environment may have primed a hierarchy of autonomous goals in the Asian group explaining the absence of cultural differences in the autobiographical memory of trauma. However, this seems unlikely given the study found cultural differences in the autobiographical memory of everyday events and self-defining memories. Furthermore, it seems unlikely that the difference in task context would impact on memory theme or on the interaction between culture and theme for memory elaboration. However, it may impact on findings like specificity. Given the pattern and size of the results with a smallish sample there is some confidence that they are robust. Nevertheless, it is recognized the importance of examining contextually effects on personal remembering which may interact with cultural dependent differences. Fourth, the international student status, often accompanied by high education and good resilience of the Asian students may have impacted on findings. This may have impacted on the absence of trauma-centered self-definition being accompanied by poorer adjustment in the Asian group. However, this seems unlikely as the Asian group scored higher on the IES-R and produced as many trauma-themed memories as the Australian group. Further, although no overall cultural differences in the type, importance, perceived impact and time of the trauma were found, the study would be improved if trauma type was more tightly controlled and matched across groups. Moreover, including only trauma types that would have the potential of meeting DSM-IV (2000) PTSD Criteria A would strengthen current clinical implications. Finally, Asian international students were considered as a single, 
interdependent population. Although, according to Hofstede and Hofstede (2004) all Asian cultures represented in this study are located on the collectivism end of the continuum and Australia on the extreme individualism side, and other studies (e.g., Wang \& Ross, 2005) have used a similar approach, it is worth incorporating a measure controlling for independence/interdependence.

To extend external validity and clinical inferences of this study, a second study examining the narratives, self-defining memories and self-concept measures in a general community sample comprised of trauma exposed adults with and without PTSD, from interdependent and independent cultures sample is needed. 


\section{CHAPTER 6}

\section{Study 2: Cultural Differences in Cognitive Appraisal Styles Utilized During Trauma and Subsequent Impact on Psychological Adjustment in a Non-Clinical Sample}

Several theorists posit that cognitive appraisal factors play a role in the maintenance of PTSD. They have linked the severity of PTSD symptomatology to specific maladaptive beliefs about the self, world and/or to the nature of the traumatic memory. A current fusion of cognitive factors maintaining PTSD is presented in Ehlers and Clark's (2000) appraisal model. This model provides possible mechanisms, other than trauma's impact on self-definition, which may be implicated in the maintenance of PTSD symptomatology and hence, may explain the maintenance of PTSD in individuals from interdependent cultures.

As outlined in the introduction, the appraisal model extends SMS in that it posits that two key processes are involved in the development and maintenance of poor psychological adjustment following trauma - individual differences in the nature of autobiographical memory for the event and its link to other autobiographical knowledge, and individual differences in the self-related appraisals of trauma and/or the trauma sequelae. They suggest a reciprocal relationship between the nature of the trauma memory and appraisals; people with poor psychological adjustment recall trauma memories biased by negative appraisals and in turn information that is consistent with these appraisals is continuously retrieved. This results in the processing of past threat as an on-going threat, which results in the appraisal of future situations as dangerous. Such appraisals generate strong emotions and arousal symptoms, which may in turn, motivate the person to engage in maladaptive strategies to control the perceived threat. These attempts to control the threat may exacerbate and maintain symptoms of PTSD.

There is now considerable empirical evidence (e.g., Dunmore et al., 2001; Ehlers, Clark, et al., 1998; Ehlers et al., 2000) supporting the appraisal model's assertion that cognitive appraisal styles contribute to perceived current threat and/or influence the nature of the autobiographical memory, suggesting cognitive appraisals are involved in the development and maintenance of PTSD. Four cognitive appraisal styles that have 
received significant attention in recent years are mental defeat, control strategies, alienation and permanent change. Therefore, these cognitive appraisal styles have been selected for this study to examine the impact of cultural differences in self-construal on cognitive appraisal styles utilized during trauma and associated psychological adjustment.

\subsection{Mental Defeat/Lack of Psychological Autonomy}

Ehlers (e.g., Dunmore et al., 2001; Ehlers et al., 2000) states mental defeat refers to the perceived loss of all psychological autonomy and is associated with the sense of no longer being human. Mental defeat refers to the threat to the self as an autonomous human being. Hence, the trauma survivor is in a mental state of 'giving up' and there is a loss of all efforts to retain one's identity as a human being with an individual will (e.g., I am an object, I was destroyed as a human being, etc.). Mental defeat or lack of psychological autonomy is seen as a cognitive appraisal style that tends to be utilized during the trauma. Mental defeat challenges the survivor's sense of worthiness and competence and results in negative views about self, such as feelings of inferiority, unworthiness and an inability to cope with future events. Hence, the survivor continues to perceive current situations as threatening and dangerous as they perceive themself as inadequate and unable to cope in current situations, and retrieve information and memories that confirm this inadequacy.

\subsection{Control Strategies}

Control strategies refer to mental plans to retain control in the trauma situation. It involves planning in one's mind or engaging in attempts to exert control in an uncontrollable situation, to minimize physical or psychological pain, to make the experience more tolerable and/or in interpersonal traumatic experiences influence the response of the assailant. These cognitive strategies need not be successful to be defined as control strategies (Ehlers et al., 2000). This cognitive style has associations with the self as control strategies refer to the cognitive activities of the self in exerting control/autonomy in the trauma (e.g., "I think that if I bite him he will release his grip," "I 
did not look at him so that I would not recognize him and he would not have to kill me", Ehlers et al., 2000, p. 46). Again control strategies are seen as a cognitive appraisal style that tends to be utilized during the trauma. Control strategies are thought to maintain PTSD symptomatology as the survivor continues to perceive current situations as threatening and dangerous by perceiving the self as unable to cope in current situations and retrieve information and memories (e.g., information and memories that the world is uncontrollable, I am not in control, a lack of mastery and agency, etc.) that confirm this inability (Ehlers et al., 2000).

\subsection{Alienation}

Alienation refers to appraisals of the enduring impact of the trauma on self in relationship with others. It is associated with relatedness and interpersonal relationships. Alienation is taken from statements about interactions with others and the survivor's feelings about such interactions. It includes not being able to relate to others, a sense of others not being able to meet the needs of the survivor and an increased sense of not being supported by the survivor's social network and/or the self not being a member of the social network (Ehlers, Clark, et al., 1998). These appraisals are generally generated post-trauma. Alienation is thought to maintain poorer trauma adjustment because survivors have on-going negative appraisals of interactions with others. People who experience alienation may withdraw from social interactions and are thus, less likely to receive social support and to correct negative beliefs about themselves and others (Ehlers et al., 2000).

\subsection{Permanent Change}

Permanent change refers to beliefs that the trauma caused permanent change for the worse in the self and personality. It also refers to trauma destroying former lives completely (e.g., a sense that one's life is over, I am damaged forever, I died on the inside, etc.). Permanent change tends to be alienation from the self and personal life goals 
(Ehlers, Clark, et al., 1998). Again, these appraisals are generally generated post-trauma (Ehlers et al., 2000).

There is evidence that these four appraisals operate in PTSD consistently with the cognitive appraisal model. A number of studies have found mental defeat to be associated with PTSD severity (Dunmore et al., 2001; Ehlers, Mayou, \& Bryant, 1998; Ehlers, Clark, et al., 1998; Ehlers et al., 2000) and mental defeat has been consistently found to be associated with persistent PTSD following assault (Dunmore et al., 2001). Ehlers et al. (2000) found that when compared to political prisoners without PTSD, political prisoners with chronic PTSD were more likely to perceive mental threat and an overall feeling of alienation and perceived negative and permanent change in their personalities or life aspirations. Ehlers, Clark, et al. (1998) found that rape survivors whose memories reflected mental defeat or the absence of mental planning/control strategies showed little improvement following exposure therapy, and, inferior outcome was associated with overall feelings of alienation and permanent change following the trauma.

\subsection{Linking Appraisal Styles to Self}

These cognitive appraisal styles have clear connections to relatedness (alienation) and autonomy (mental defeat, control strategies). Although permanent change is not clearly associated with either aspect of self, it is proposed that permanent change may reflect self-definition/identity change as examined in the previous chapter. In Study 1, post-trauma self-definition/identity change was culturally specified. Such a finding supported the theory of self-construal's (Markus \& Kitayama, 1991) conceptualization of self-definition; in independent cultures self-definition is consistent across social contexts, while in interdependent cultures the self is defined by the current social context. Additionally, the finding supported the theory of self-consistency (Suh, 2000); in independent cultures self-consistency is valued, while in interdependent cultures selfflexibility across contexts is valued. This empirical finding in Study 1, alongside these two theoretical stances, suggests that a sense of (permanent) change in self aligns with the independent self-construal. 
Chapter 3 outlined, both theoretically and empirically, that cultural differences in self-construal impact on the way in which events, situations and life encounters are appraised, and on associated affective responses. The conceptual framework chapter combined the literature indicating cultural differences in self-construal impact on appraisals and subsequent affective responses with Ehlers and Clark's appraisal model. Based on this, the current study proposes to explore the impact of cultural differences in self on these cognitive appraisal styles of trauma and subsequently the impact on adjustment to trauma.

The conceptual framework of the modified SMS suggests that a traumatic event activates an autonomous goal hierarchy or working self, regardless of the culturally dominant self. It is thought that in order to maintain an autonomous sense of self there is a universal foregrounding of autonomy in remembering traumatic events. This prediction was supported in the findings of Study 1. Extending this, it is expected there will be a universal foregrounding of autonomy in the cognitive appraisals (mental defeat and control strategies) generated during the trauma. This prediction is based on the following three assertions. First, trauma activates an autonomous self-construal and research (e.g., Mesquita \& Walker, 2003) suggests self-construal impacts on the way in which one appraises situations. Second, there seems to be a promotion of an autonomous sense of self during trauma and in the remembering of trauma. Third, Ehlers and Clark propose a reciprocal relationship between the nature of the trauma memory and the appraisals of the trauma. Specifically, if the trauma memory is predominately autonomous regardless of the dominant self-system, appraisals will reflect this dominance and be centered round the theme of autonomy (e.g., I was powerful, I conquered, I lost control, I am unable to cope, etc.).

Research has demonstrated that those from individualistic cultures experience poor mental health when personal control over their environment is perceived to have diminished (e.g., Mesquita \& Karasawa, 2002; Sato, 2001). In contrast, research (e.g., Mesquita \& Walker, 2003) has shown personal control has limited applicability in collectivistic cultures. Additionally, it is suggested that trauma memories biased by negative autonomous appraisals have the potential to threaten the independent self. However, the interdependent self is less challenged by negative autonomous appraisals. 
According to Ehlers and Clark (2000) appraising current situations as an on-going internal threat to the self is accompanied by PTSD symptoms. According to Ehlers and Clark, the perceived threat prompts behavioral and cognitive responses that aim to "reduce perceived threat and distress in the short-term, but have the consequence of preventing cognitive change and therefore maintaining the disorder" (p. 320). Therefore, it is predicted that despite the absence of cultural differences in the appraisals generated during trauma, the impact of these appraisals on psychological adjustment will differ culturally. Specifically, following trauma it is suggested that the fewer the autonomous oriented cognitive appraisal styles (i.e., more mental defeat and less control appraisals) utilized during trauma the greater the disrupted psychological adjustment for Australians (individualistic culture) when compared to Asians (collectivistic culture).

Once the trauma event is over, along with the trauma associated cues that prime an autonomous mindset, it is predicted that Mesquita and Walker's (2003) theoretical position of cultural differences in self-construal impacting on the way in which events, situations and life encounters are appraised will be evident. Those from individualistic cultures will appraise the trauma in terms of personal control and autonomy, while for collectivistic cultures personal control and autonomy will have little relevance; rather emphasis will be given to the interdependence of an individual and their social environment. Specifically, the cognitive appraisal styles (alienation and permanent change) utilized to generate appraisals of the events following trauma will reflect the dominant conceptual self. Hence, it is proposed that the post-trauma appraisals of those from interdependent cultures will emphasize negative relatedness appraisals (i.e., alienation), whilst those from independent cultures will emphasize negative autonomous appraisals (i.e., permanent change).

Regarding psychological adjustment, Sato (2001) theorizes that poor mental health results when individuals with a strong independent self-construal perceive diminished levels of personal control over their environment, while for individuals holding an interdependent self-construal poor mental health results when there is perceived alienation or isolation. It is predicted that Asians with disrupted relatedness appraisals will have poorer adjustment to the trauma given the threat these appraisals pose to the interdependent conceptual self. Likewise, Australians with disrupted 
autonomous appraisals will have poorer adjustment to the trauma given the threat these appraisals pose to the independent conceptual self.

\subsection{Aims and Hypotheses}

This study attempts to explore the impact of cultural variations in self on the cognitive appraisals (mental defeat and control strategies, alienation and permanent change) and their relationship to psychological adjustment. It does this by re-analyzing the trauma narratives generated in Study 1 with a focus on the impact of cultural variations in self on these four cognitive appraisals and the consequence of this on psychological adjustment following trauma. The following is predicted:

$1 /$ there will be no cultural differences in the cognitive appraisal styles (mental defeat/ lack of psychological autonomy, control strategies) utilized during trauma as an autonomous goal hierarchy is employed during trauma and hence, autonomous cognitive appraisal styles are employed,

2/ despite an absence in cultural variation in the cognitive appraisal styles utilized during the trauma, the impact of these cognitive styles on psychological adjustment will differ culturally. Specifically, for Australians the greater the mental defeat and the fewer the control strategies employed during the trauma the poorer the psychological adjustment. This relationship will not be as significant for the Asian group,

3/ there will be cultural differences in the cognitive appraisal styles (alienation, permanent change) generated post-trauma as the conceptual self and working self are realigned. Specifically, Asians will have greater alienation appraisals than Australians and Australians greater permanent change appraisals that Asians in their trauma memories, and

4/ there will be cultural variation in the impact of these cognitive appraisal styles (alienation, permanent change) on psychological adjustment. Specifically, for Australians the greater the permanent change appraisals generated the poorer the psychological adjustment. This relationship will not be as significant for the Asian group. Furthermore, for Asians the greater the number of alienation appraisals generated, the poorer the 
psychological adjustment. This relationship will not be as significant for the Australian group.

\subsection{Method}

\subsubsection{Participants}

The participants were the same sample as that used in Study 1.

\subsubsection{Procedure}

This study utilized the trauma narratives and corresponding disrupted adjustment to trauma measure (Impact of Event Scale-Revised; Weiss \& Marmar, 1997) collected in Study1.

\subsubsection{Scoring}

Narratives were scored based on the coding developed in the 10-page manual by Ehlers, Clark, and Foa (received by personal correspondence, cited in Ehlers, Clark, et al., 1998) and Ehlers et al. (2000). Following Ehlers, Clark, et al. (1998) narratives were read three times. The first reading was to familiarize the raters with the memory. The raters then read the transcripts again highlighting any material relevant to the concepts of mental defeat, control strategies, alienation and permanent change. On the third reading raters gave an overall rating using the following criteria.

\section{Mental defeat.}

Mental defeat was defined as the perceived loss of psychological autonomy, the sense of no longer being human, the threat to the self as an autonomous human being, the state of mentally giving up and the loss of all efforts to retain one's identity as a human being with an individual will. Evidence for mental defeat comes from direct comments about the survivor's perception that they gave up in their own mind, were completely defeated, felt completely at the will of the assailant or cause of trauma, the loss of being a person with their own will, no longer feeling like a human being and so forth. Mental defeat is rated conservatively and goes beyond helplessness and hopelessness. Autonomous frame of mind is the opposite of mental defeat and reflects a retainment of a 
sense of freedom of mind and maintaining their will, convictions and/or character. Each participant's narrative received a mental defeat score of either -2 (strong evidence of autonomous frame of mind during trauma), -1 (some evidence of autonomous frame of mind during trauma), 0 (no evidence of either autonomous frame of mind during trauma or loss of psychological autonomy during trauma), 1 (some evidence of loss of psychological autonomy) or 2 (strong evidence of loss of psychological autonomy). There were no examples of mental defeat. Given, the study did not find any evidence of mental defeat, the mental defeat appraisal style tended to be a measure of the level of psychological autonomy in the trauma memory. Thus, mental defeat will be referred to as psychological autonomy throughout. An example of high levels of psychological autonomy was "I decided I would live my life the way I wanted" (Australian) (rating -2).

\section{Control strategies.}

Control strategies was coded as the overall level of thinking about or planning in one's mind about what might be able to be done to minimize physical or psychological harm, to make the experience more tolerable, or to influence the situation. Evidence for control strategies was taken from planning thoughts, statements and actions that were taken intentionally in an attempt to exert control over the situation. Each participant's narrative received a control strategies score as either 0 (no evidence of control strategies), 1 (little evidence of control strategies), 2 (some evidence of control strategies), 3 (moderate evidence of control strategies) or 4 (strong evidence of control strategies). Examples of control strategies include: "I was going to ensure that the 'other' party to the affair didn't win. I maintained the relationship for another 6 months, more out of competitive spirit than because I loved her" (Australian) (rating 3). "During a bush fire I collected all my precious belongings, and put them in the car... I went around taking down all the curtains, moving furniture away from under the window...put tennis balls in the down pipes, so the gutters could be filled with water" (Australian) (rating 3).

\section{Alienation.}

Alienation was coded as a general overall feeling in the narrative that the participant felt alienated from the world and others. This was taken from participants' feelings with others after trauma and their feelings about these interactions. It needed to be clear that participants viewed these interactions and people in a negative way. It also 
included not being able to interrelate with people after trauma or that others failed to meet their needs. Evidence against alienation was taken from statements about a sense of support and safety provided by others. Each participant's narrative received an alienation score of either -2 (strong evidence against alienation, i.e., connectedness), -1 (some evidence against alienation, i.e., connectedness), 0 (no evidence against or for alienation), 1 (some evidence of alienation) or 2 (strong evidence of alienation). Examples of alienation included statements such as "I will live alone” (Asian) (rating 1), “ I felt very alone" (Australian) (rating 2), "I stayed in isolation...rarely going out...withdrawing from everyone" (Asian) (rating 2), "I locked myself in my room, didn't want to talk with anyone, even my family" (Asian) (rating 1) and " No one understood me" (Australian) (rating 1). An example of evidence against alienation included statements such as "People came to help. Lots of people which is good...it's amazing how many people came to do whatever they could do to help" (Australian) (rating-2).

\section{Permanent change.}

Permanent change was coded as an overall sense that participants viewed their life as having been changed forever in a negative way. Evidence against permanent change was a sense that participants saw the event as an isolated bad experience or participants felt that they had grown or developed from the experience. Each participant's narrative received a permanent change score of either 0 (no negative changes in personality, life goals, future of life), 1 (little evidence of changes in personality, life goals, future of life), 2 (some evidence of changes in personality, life goals, future of life), 3 (moderate evidence of changes in personality, life goals, future of life) or 4 (strong evidence of changes in personality, life goals, future of life). Examples of permanent change included statements such as "Ever since then I think that experience changed my life" (Australian) (rating 2), "That event has made me very emotional which still I can't think of" (Asian) (rating 2) and "The impact was enormous, and I still struggle with it" (Australian) (rating 2). 


\subsubsection{Reliability}

A second Chinese independent rater coded $20 \%$ of the trauma narratives. Discrepancies between raters were resolved through discussion. Raters were blind to the cultural group of participants. Kappa coefficients were adequate for the psychological autonomy $(\kappa=.74)$, control strategies $(\kappa=.87)$, alienation $(\kappa=.88)$ and permanent change $(\kappa=.69)$.

\subsection{Results}

\subsubsection{Participant Characteristics}

Participant characteristics are outlined in Study 1. As outlined in Study 1, Asians reported significantly higher on the IES-R than Australians.

\subsubsection{Preliminary Analysis}

As stated in Study 1, there was no significant difference between Australians and Asians reports of how traumatic they found the trauma event, event impact, or time of event.

\subsubsection{Comparative Analysis of Narratives}

Independent one-tailed $t$ tests were conducted to explore cultural differences in the four cognitive appraisal styles. Given, Asians scored significantly higher on the IES$\mathrm{R}$ than did Australians, the IES-R was used as a covariate in the following analyses.

Asians $(M=-.17, S D=.82)$ did not differ from Australians $(M=-.19, S D=.75)$ in level of psychological autonomy, $t(48)=.06, p=.48, C 1.95=-.47, .42, d=.03$. Asians $(M=1.08, S D=1.06)$ did not differ from Australians $(M=1.42, S D=1.14)$ in control strategies, $t(48)=.67, p=.25, C I .95=-.29, .97, d=.30$. Asians $(M=.50, S D=1.10)$ reported significantly more alienation in their trauma narratives than did Australians $(M=$ $-.54, S D=1.07), t(48)=2.22, p=.02, C 1.95=.42,1.66, d=.78$. Australians $(M=.96, S D$ $=.77$ ) tended to have more appraisals of permanent change in their trauma narratives than $\operatorname{did}$ Asians $(M=.63, S D=.71), t(48)=1.60, p=.06, C 1.95=-.09, .76, d=.45$. 


\subsubsection{Psychological Adjustment}

Table 6.1 shows the correlation coefficients between the four cognitive appraisal styles and self-reported adjustment to the trauma (IES-R) for Australians and Asians, respectively. Table 6.1 also shows the $Z$ difference score (and associated $p$ value) between the two cultural groups for each correlation coefficient.

Small, negative relationships were found between lack of psychological autonomy and problematic adjustment for Australians and no relationship between a lack of psychological autonomy and problematic adjustment was found for Asians. Overall, for Australians, small to medium negative correlations were found between control strategies and problematic adjustment but no relationship was found between control strategies and hyperarousal symptoms. No relationship between control strategies and psychological adjustment was found for the Asian group. Overall, while no relationship was found between alienation and problematic adjustment for the Australian group, moderate, positive correlations were found between alienation and problematic adjustment for the Asian group. No relationship between permanent change and problematic adjustment was found for either cultural group. None of the correlation coefficients differed significantly between the two cultural groups. However, some (e.g., control strategies and avoidance symptoms; alienation and avoidance symptoms; and alienation and total IES-R) of the differences were approaching significance and it is thought that a lack of findings may be due to a lack of statistical power (i.e., small sample sizes). 
Table 6.1

Correlation Coefficients between the Four Cognitive Appraisal Styles and Self-Reported Adjustment to Trauma for the Asian and Australian Group and Z Difference Scores between Cultural Groups' Correlation Coefficients.

\begin{tabular}{|c|c|c|c|c|c|c|c|c|c|c|c|c|c|c|c|c|}
\hline & \multicolumn{4}{|c|}{$\begin{array}{c}\text { Lack of Psychological } \\
\text { Autonomy }\end{array}$} & \multicolumn{4}{|c|}{ Control Strategies } & \multicolumn{4}{|c|}{ Alienation } & \multicolumn{4}{|c|}{ Permanent Change } \\
\hline & Aust. & Asian & $Z_{\text {diff }}$ & $P$ & Aust. & Asian & $Z_{\text {diff }}$ & $p$ & Aust. & Asian & $Z_{\text {diff }}$ & $p$ & Aust. & Asian & $Z_{\text {diff }}$ & $p$ \\
\hline Avoidance & -.12 & .19 & 1.04 & .30 & -.31 & .17 & 1.63 & .10 & .04 & $.49 *$ & 1.64 & .10 & .06 & .06 & .00 & 1.00 \\
\hline Intrusions & -.11 & .13 & .80 & .42 & -.20 & -.06 & .47 & .64 & -.02 & .25 & .91 & .36 & .11 & .01 & .33 & .74 \\
\hline Hyperarousal & -.25 & -.02 & .91 & .44 & -.03 & .02 & .17 & .87 & .04 & .34 & 1.04 & .30 & -.01 & .10 & .37 & .71 \\
\hline Total & -.16 & .14 & 1.00 & .32 & -.23 & .04 & .91 & .36 & .02 & $.42 *$ & 1.42 & .16 & .07 & .06 & .03 & .97 \\
\hline
\end{tabular}




\subsection{Discussion}

The objective of this study was to explore the impact of cultural differences in self on four relatively well-researched cognitive appraisal styles of trauma and the subsequent impact on psychological adjustment to trauma and in doing so to explore the cultural flexibility of Ehlers and Clark's (2000) appraisal model. However, the results were inconsistent and thus, at best, provide a very preliminary analysis of this objective.

The first hypothesis was supported. There were no cultural differences in the cognitive appraisal styles (psychological autonomy, control strategies) utilized during trauma. This supports the conceptual framework's position that a traumatic event by its nature primes the autonomous self-construal, regardless of the culturally dominant selfconstrual. The primed autonomous self-construal seems to be utilized in governing appraisals at the time of the trauma. Therefore, there seems to be a universal foregrounding of autonomy not only in remembering traumatic events (Study 1) but also in appraisals generated at the time of the trauma event.

Due to inconclusive findings, the second hypothesis could not be supported. It was predicted that despite an absence in cultural variation in the cognitive appraisal styles utilized during the trauma the impact of these cognitive styles on psychological adjustment would differ culturally. Supporting the hypothesis, it was found that for the Australian group fewer control strategies was associated with poorer psychological adjustment, while for the Asian group there was no relationship between either the level of psychological autonomy or control strategies and psychological adjustment. Such a finding aligns with research (e.g., Mesquita \& Karasawa, 2002) that has demonstrated that individualistic cultures experience more positive affect when they appraise a situation as under personal control, while this was not the case in collectivistic cultures. The finding also aligns with Sato's (2001) suggestion that for individualistic cultures poor mental health may result when there is perceived diminished levels of personal control over the environment, while this seems not to be the case for collectivistic cultures. However, undermining this support, there was some evidence to suggest that for the Australian group the greater the psychological autonomy appraisals in the memories the greater the problematic adjustment. Additionally, there was no evidence to suggest 
that the relationship between these autonomous cognitive appraisal styles and psychological adjustment were less marked in the Asian group when compared to the Australian group, as the correlation coefficients did not differ significantly between the two cultural groups.

The third hypothesis was supported. That is, there were cultural differences in the cognitive appraisal styles (alienation and permanent change) generated post-trauma suggesting the conceptual and working selves are re-aligned. Specifically, Asians had greater alienation appraisals than Australians and Australians had greater permanent change appraisals than Asians in their trauma memories. This supports the notion that once the trauma event is over, along with the trauma associated cues that prime an autonomous self-construal, cultural differences in self-construal impact on the way in which events, situations and life encounters are appraised. Those from individualistic cultures aim to appraise the trauma and its sequelae in terms of personal control and agency, while for collectivistic cultures personal agency has very limited applicability, rather emphasis in the appraisals of the trauma and its sequelae is given to the interdependence of an individual and their social environment.

The final hypothesis was only partially supported. There was evidence suggesting a relationship between greater alienation appraisals and poorer psychological adjustment in the Asian group. This supports Sato's (2001) assertion that in collectivistic cultures poor mental health may result when individuals perceive alienation or isolation. However, this relationship was not more marked (as indicated by no cultural differences in correlation coefficients) in the Asian group when compared to the Australian group. Results, however, were approaching significance and hence, it is thought that a lack of a significant difference may be the result of a lack of statistical power. Unexpectedly, there was no evidence suggesting a relationship between permanent change and psychological adjustment for either the Australian or Asian group.

The inconsistent findings in terms of the impact of cultural differences in selfconstrual on the relationship between the cognitive appraisal styles and psychological adjustment are thought to be the result of the nature and size of the sample. Ehlers' (e.g., Ehlers et al., 2000) research exploring the relationship between these cognitive appraisal styles and psychological adjustment has been conducted solely on trauma survivors with 
PTSD and moreover, trauma survivors of severe interpersonal traumas (e.g., rape and torture). Therefore, it seems plausible that the sample being comprised of university students, of which few would meet PTSD diagnosis as outlined in DSM-IV (1994), would have impacted significantly on the relationship between the appraisals and psychological adjustment.

The limitations of this study are acknowledged. The sample is a relatively small, non-clinical sample limiting the clinical inferences and statistical power of the results. Furthermore, there could be no exploration of cultural differences in the cognitive appraisal styles between those who develop PTSD and those who do not, as the IES-R is an inadequate measure of a PTSD diagnosis. Hence, this study failed to explore cultural differences in these cognitive appraisal styles between those with and without PTSD. Second, as in the other studies, these results must be considered in the context of possible differences in intercultural context as compared to intracultural context, especially the impact this may have on alienation. It was found that the Asian group had significantly more alienation appraisals than the Australian group. This may be the result of the Asian group being comprised of international students, which may be associated with feelings of isolation and alienation and thus, may prime alienation in memory retrieval. However, this does not seem likely as participants were retrieving a trauma memory which on average happened years ago; there seemed to be no cultural differences in comments of alienation in the everyday narratives found in Study 1; and Study 1 found that Asian students actually tended to retrieve everyday and self-defining memories that focused on social connectedness. Finally, trauma type may have impacted on findings as Ehlers' (e.g., Ehlers et al., 2000) work has been conducted using participants who have endured relatively severe, interpersonal traumas of often relatively long duration. This was not controlled for in this study and hence, could not be explored.

In sum, despite its limitations this study provides support for cultural similarities in the cognitive appraisal styles (psychological autonomy, control strategies) utilized during trauma supporting the assertion that a traumatic event by its nature primes the autonomous self-construal, and thus appraisals generated at the time of the trauma are governed by an autonomous self-construal. However, it was found post-trauma the conceptual self and working self re-align. That is, once the trauma event is over, along 
with the trauma associated cues that prime an autonomous self-construal, cultural differences in self-construal impact on the way in which events, situations and life encounters are appraised. Those from individualistic cultures will endeavor to attribute the trauma and its sequelae to being under personal control and agency, while for collectivistic cultures personal agency has very limited applicability, rather emphasis is given in appraisals to the interdependence of an individual and their social environment. Specifically, Asians had greater alienation appraisals than Australians and Australians greater permanent change appraisals that Asians in their trauma memories. The findings related to psychological adjustment were inconsistent. There was no convincing evidence suggesting the level of autonomous appraisals impacts on psychological adjustment more in the Australian than Asian group. There was some evidence suggesting a relationship between alienation and poorer psychological adjustment in the Asian group. However, these analyses were very preliminary given the nature and size of the sample. The most consistent finding in this study was the highlighted need to explore similar hypotheses in a community sample comprised of trauma survivors with and without PTSD from independent and interdependent culture. 
Series 2

Cultural Differences in Autobiographical Memory in Posttraumatic Stress Disorder: Extending the Ecological Validity, and Theoretical and Clinical Implications of Series 1. 


\section{CHAPTER 7}

\section{Study 3: Cultural Differences in Autobiographical Memory of Trauma and Personal Identity in Posttraumatic Stress Disorder}

The ecological validity and clinical implications that could be drawn from Study 1 were limited as participants were students with trauma exposure but few would have met the criteria for PTSD as outlined in the DSM-IV (1994). In addition, the validity of inferences about cultural differences was unclear because cultural groupings were based solely on ethnicity without a validating measure of independence/interdependence. Study 3 is comprised of three sub-studies which examine if PTSD moderates cultural differences in autobiographical memory of trauma and trauma-themed personal identity. To extend ecological validity and clinical implications this study tests similar predictions to that of Study 1 in a sample comprised of trauma survivors with and without PTSD from independent and interdependent cultures. Specifically the study aims to investigate, firstly, whether PTSD moderates the impact of cultural differences in self on the autobiographical remembering of everyday and trauma events. Secondly, it aims to explore whether the proposed impact of trauma on the conceptual self is evident in the retrieval of selfdefining memories. Thirdly, Study 3 examines whether self-change towards traumacentered identity in the aftermath of trauma is culturally specified. Finally, Study 3 aims to replicate the findings of Study 1 regarding the content and structure of autobiographical memories of everyday events, trauma events and self-defining memories.

\subsection{Relationship between Cultural Differences in Autobiographical Memory and PTSD}

The first objective of Study 3 is to explore whether PTSD moderates the impact of cultural differences in self on the autobiographical remembering of everyday and trauma events. The proposed conceptual framework modified SMS enabling it to make predictions regarding the impact of cultural variations in self on the nature of autobiographical memory. The framework did not, however, account for the relationship between cultural differences in the nature of autobiographical remembering and PTSD, as it was believed that the initial modifications to SMS 
needed to be firstly assessed. Given Study 1 confirmed the validity of the initial modifications to SMS, this thesis will now outline specific proposals about the relationship between cultural differences in the nature of autobiographical memory of trauma and PTSD.

\subsubsection{Centrality of the Trauma Memory}

Conway (2005) theorizes that the conceptual self impacts on schema knowledge. Markus and Kitayma (1991) posit that cultural differences in selfconstrual govern schema. Nelson and Fivush (2004) attest to the socio-cultural emergence of autobiographical memory which enables individuals to possess knowledge about culturally appropriate levels of autonomy in autobiographical remembering. Wang and Conway (2004) claim that such knowledge carries over into adulthood. Mesquita and Walker (2003) argue that there are cultural expectations as to appropriate levels of personal control and agency in an emotional event, and assert that situations which violate these cultural expectations and norms can have detrimental effects for individuals. By combining these five theoretical stances, it is hypothesized that people have schema-driven expectations as to appropriate levels of autonomous orientation in autobiographical remembering which is derived from the conceptual self. Specifically, for those from independent culture, an emphasis on an independent conceptual self results in schema-driven expectations of high levels of autonomous orientation in autobiographical remembering, as high levels of autonomous orientation reaffirm personal control, mastery, uniqueness and autonomy (Wang \& Conway, 2004). In contrast, for those from interdependent culture an emphasis on a interdependent conceptual self results in schema-driven expectations of low levels of autonomous orientation in autobiographical remembering, as autonomous orientation is not valued and has the potential to undermine group harmony, 'fitting in' and a sense of interdependence (Wang \& Conway, 2004).

Research (e.g., Brewer \& Treyens, 1981; Brown \& Kulik, 1977; Hunt \& McDaniel, 1993; Rubin \& Kozin, 1984) has found that information that deviates from schema-driven expectations results in enhanced memory of the information, and distinctive and emotional events are generally highly accessible and clearly remembered compared to less significant life experiences (e.g., Berntsen \& Rubin, 2007; Hunt \& McDaniel, 1993; McGaugh, 2003). According to Berntsen and Rubin (2007) highly accessible autobiographical memories often become the reference 
points for the organization of autobiographical memories of less significant events. Therefore, they suggest, given information in the trauma memory typically deviate from schema-driven expectations, the memory of the trauma event is enhanced, and thus, trauma autobiographical memories may form "reference points for the organization of other less significant experiences" (p. 418). They propose that if trauma memories do become central to the organization of autobiographical memory, it is likely to result in poor posttraumatic psychological adjustment, as in Ehlers and Clark's (2000) terms current situations are perceived to be a serious threat.

The conceptual framework asserted, supported by the findings of Study 1, that when a trauma event occurs it predominately activates an autonomous working self. However, despite the activation of an autonomous working self and universal foregrounding of autonomy, Study 1 demonstrated individual differences in the level of autonomous orientation in the trauma memory. It is proposed that for some trauma survivors the level of autonomous orientation in the autobiographical remembering of trauma violates schema-driven expectations of appropriate levels of autonomy in autobiographical remembering. Specifically, for those from independent culture low levels of autonomous orientation, and for those from interdependent culture high levels of autonomous orientation, may violate schema-driven expectations. Such violations result in enhanced memory of the trauma event, which in turn, following Berntsen and Rubin's rationale, becomes the reference point for autobiographical memory. This is likely to result in poor posttraumatic psychological adjustment as, according to Ehlers and Clark, current situations are perceived to be a serious threat to the self. Therefore, it is predicted that trauma survivors from independent cultures with PTSD will have less autonomous orientation in their trauma memories than those from independent cultures without PTSD, while trauma survivors from interdependent cultures with PTSD will have greater autonomous orientation in their trauma memories than those from interdependent cultures without PTSD.

It is proposed that centrality of the trauma memory in autobiographical memory has the potential to impact on the functional role of autobiographical memory, threaten the conceptual self, and become central to identity, self-concept and self-definition. 


\subsection{Threat to Conceptual Self}

\subsubsection{Impact on the Functional Role of Autobiographical Memory}

It is proposed that one potential outcome of centrality of the trauma memory is the functional role of autobiographical memory is disrupted by the trauma memory. According to Berntsen and Rubin (2007), the trauma memory, as the reference point for the organization of autobiographical memory, is likely to result in everyday events, which would normally be perceived as neutral or positive, becoming associated with the trauma memory. Additionally, it is believed that aspects of the content and structure of everyday autobiographical memories become associated with, and reflect, the content and structure of the trauma autobiographical memory.

Wang and Conway (2004) assert, as shown in Figure 7.1, that autobiographical memories are instrumental in the development, expression and maintenance of the self. Therefore, it is hypothesized that if the trauma memory becomes the cognitive reference point for other autobiographical memories, the level of autonomous orientation in the trauma memory is likely to impact on the expression, development and maintenance of the self, or in terms the of the SMS, the conceptual self.

Macro cultural level

Micro individual level

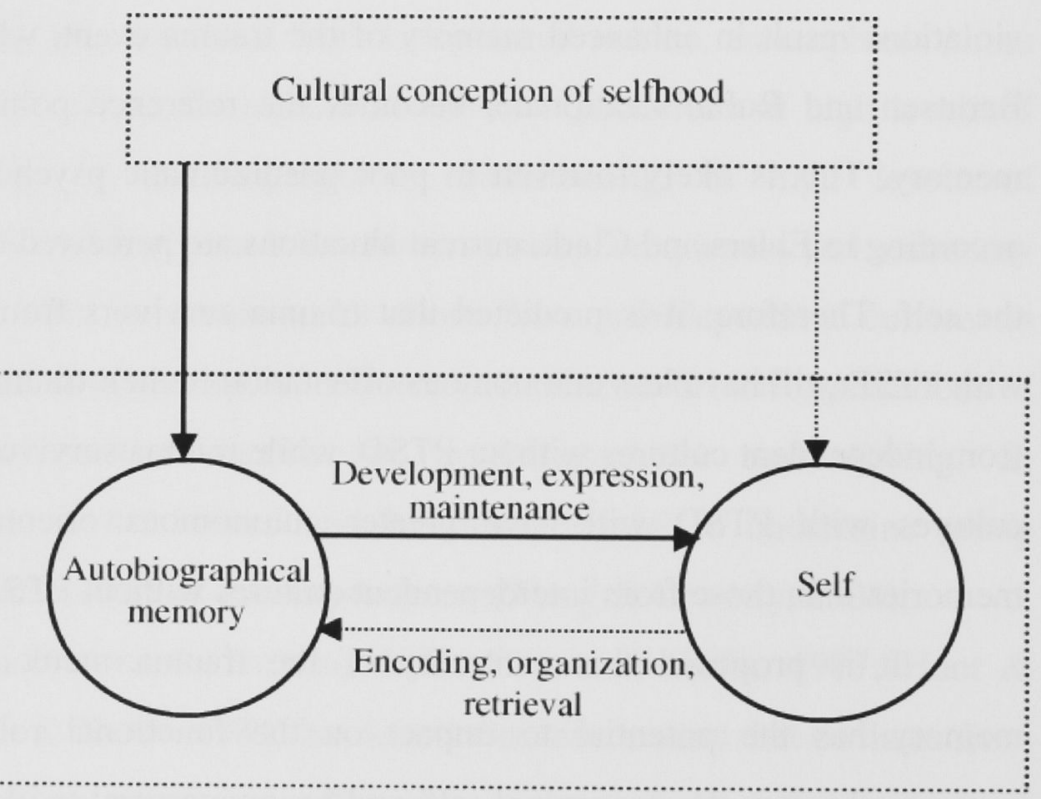

Figure 7.1 Relations between culture, self and memory. From Wang, Q., \& Conway, M. A. (2004). The stories we keep: Autobiographical memory in American and Chinese middle-aged adults. Journal of Personality, 72, 914. Copyright 2004 by Blackwell Publishing. Reprinted with permission. 
For independent cultures, a central trauma autobiographical memory with low levels of autonomous orientation is thought to result in the development, expression and maintenance of a self lacking autonomy, personal control, uniqueness and selfassertion. Current theory (e.g., Beck, 1983; Blatt, 1990; Markus \& Kitayma, 1991, 1994; Mesquita \& Walker, 2003; Sato, 2001) suggests perceived or actual diminished autonomy and personal control can result in detrimental outcomes for individuals with an independent self-construal. Sato (2001) suggests a person lacking autonomy is seen as "dysfunctional" in independent societies. This may result in social alienation, which Ehlers et al. (2000) claim maintains PTSD. In contrast, for interdependent cultures, a central trauma autobiographical memory with high levels of autonomous orientation results in the development, expression and maintenance of a self emphasizing autonomy, personal control, uniqueness and self-assertion. Aspects of the self deemed culturally inappropriate in interdependent cultures and undermine relatedness and interdependence. Sato (2001) suggests interdependent societies are less tolerant of a person emphasizing autonomy, and such a person is seen as "dysfunctional". Such a trauma survivor may thus, be more prone to alienation and social isolation. It is argued (e.g., Beck, 1983; Blatt, 1990; Markus \& Kitayma, 1991, 1994; Mesquita \& Walker, 2003; Sato, 2001) that for those with an interdependent self-construal, poor psychological adjustment can occur when there is perceived or actual increased autonomy, alienation, rejection by significant others and a lack of social support.

According to Ehlers and Clark (2000), trauma survivors that appraise current situations as an on-going threat tend to have PTSD. They claim threat "very commonly, [is] internal (e.g. a threat to one's view of oneself as a capable/acceptable person who will be able to achieve important life goals)" (p. 320). One potential means by which trauma survivors may appraise current situations as an on-going internal threat and thus, as a threat to one's view of oneself as a capable/acceptable person who will be able to achieve important life goals, is by the development, expression and maintenance of a culturally inappropriate self. Specifically, the development of the independent self in terms of a lack of autonomy and the development of the interdependent self in terms of an emphasis on autonomy, may act as an internal threat. Ehlers and Clark posit that such threat to the self is accompanied by PTSD symptoms. The thesis proposes, however, that Ehlers and Clark notion of internal threat (i.e., a threat to "one's view of oneself") is relatively individualistic as it 
focuses on private aspects of self. It is suggested 'internal threat' be extended to include threat to the perceived or actual social view of self as a capable/acceptable person who will be able to achieve important life goals.

The second objective of this study is to investigate whether this proposed impact of the level of autonomous orientation in the trauma memory on the development, expression and maintenance of the conceptual self is reflected in selfdefining memories (i.e., memories that define the self and provide a constructive approach to exploring change in the conceptual self). It is predicted that trauma survivors from independent cultures with PTSD will have less emphasis on autonomy in their conceptual self, as reflected in their self-defining memories, than trauma survivors from independent cultures without PTSD, while trauma survivors from interdependent cultures with PTSD will have greater emphasis on autonomy in their conceptual self, as reflected in their self-defining memories, than trauma survivors from interdependent cultures without PTSD.

\subsubsection{Impact on other Autobiographical Memories}

Moreover, as Figure 7.1 illustrates, Wang and Conway (2004) theorize a reciprocal relationship between autobiographical memory and the self. Namely, while autobiographical memories develop, express and maintain the self, the self is instrumental in the encoding, organization and retrieval of autobiographical memories. Therefore, it seems likely that threat to the conceptual self will effect ongoing encoding, organization and retrieval of other autobiographical memories, such as memories of everyday events. This impact of the conceptual self on on-going autobiographical remembering is thought to maintain threat to the conceptual self. Therefore, it is predicted that trauma survivors from independent cultures with PTSD will have less autonomous orientation in not only their trauma memories but also in their everyday memories than trauma survivors from independent cultures without PTSD, while trauma survivors from interdependent cultures with PTSD will have more autonomous orientation in not only their trauma memories but also their everyday memories than trauma survivors from interdependent cultures without PTSD. 


\subsection{Trauma Central to Identity}

The third objective of Study 3 is to examine whether self-change towards trauma-centered identity in the aftermath of trauma is culturally specified. A final proposed potential outcome of the trauma memory becoming the cognitive reference point for other autobiographical memories is that trauma becomes central to identity, self-concept and self-definition. Berntsen and Rubin propose that the way in which life stories, and thus autobiographical memories, are composed is closely related to self-understanding and identity, and the trauma memory may anchor and stabilize self-conceptions. Conway (2005) claims that autobiographical memory validates and supports the self, and the conceptual self is comprised of the life story. Wang and Conway (2004) assert that autobiographical memory is a constituent of identity and the self. Conway and Holmes (2004) suggest that autobiographical memory defines the self. Therefore, it is proposed that centrality of the trauma autobiographical memory is most likely to be regarded as a central component of personal identity and the self.

As outlined in Study 1, several theories propose (SMS, Conway, 2005 and centrality of trauma approach, Berntsen \& Rubin, 2006, 2007), and empirical research supports (e.g., Sutherland \& Bryant, 2006), that an outcome of trauma for those with PTSD is a shift in self-concept/conceptual self/identity towards a trauma centered self and identity. Both the SMS and centrality of trauma approach posit that selfcoherence and self-consistency is instrumental in this self-change. However, as outlined in Study 1, theory and research (Suh, 2000, 2002) have demonstrated that self-consistency needs and self-definition are culturally variable. In considering this research, supported by the findings of Study 1, it is proposed that the determining factor as to whether the trauma becomes central to identity is the requirement for selfconsistency. That is, if the trauma survivor has a requirement for self-consistency and is defined by a set of stable internal attributes, it is likely that a trauma memory, central to the life story and other autobiographical memories, results in identity or conceptual self change. Identity or conceptual self change will threaten the conceptual self, as the conceptual self becomes centered round trauma.

In sum, it is predicted that the centrality of the trauma memory can become central to identity and thus, alter and threaten the conceptual self. However, a need for self-consistency is instrumental in self-change. Therefore, it is hypothesized that for 
independent cultures, cultures that value self-consistency, trauma is likely to be central to identity in those with PTSD when compared to those without PTSD, while for interdependent cultures trauma-centered identity is not likely to differentiate between those with and without PTSD.

\subsection{Sub-Studies}

\subsubsection{Study $3 a$}

Study $3 \mathrm{a}$ investigates whether PTSD moderates the impact of cultural differences in self on the autobiographical remembering of everyday and trauma events by exploring the trauma and everyday narratives of trauma survivors with and without PTSD from independent and interdependent cultures. First, it is predicted that cultural variations will be evident in the autobiographical remembering of everyday events, while the autobiographical remembering of trauma will be predominately autonomous. Second, it is predicted those from independent cultures with PTSD will have less autonomous orientation in their trauma and everyday memories when compared to independent culture without PTSD. However, those from interdependent cultures with PTSD will have more autonomous orientation in their trauma and everyday memories when compared to interdependent culture without PTSD. Additionally, Study 3 a aims to replicate the findings of Study 1 regarding the contents and structure of autobiographical memories of everyday events and trauma events.

\subsubsection{Study $3 b$}

Study 3 b explores whether the proposed impact of trauma on the conceptual self is evident in the retrieval of self-defining memories. Given the self-defining memory task is believed to be a constructive approach to assessing change in selfconcept (Sutherland \& Bryant, 2006), Study 3b uses such a task to investigate whether level of autonomous orientation in the autobiographical remembering impacts on the conceptual self. Study $3 b$ tests this prediction by exploring the selfdefining memories of trauma survivors with and without PTSD from independent and interdependent cultures. It is predicted those from independent cultures with PTSD will have less autonomous orientation in their self-defining memories when compared to those from independent culture without PTSD. However, those from interdependent cultures with PTSD will have more autonomous orientation in their 
self-defining memories when compared to those from interdependent culture without PTSD. Additionally, Study $3 \mathrm{~b}$ aims to replicate the findings of Study 1 regarding the contents and structure of self-defining memories.

\subsubsection{Study $3 c$}

Study $3 c$ examines whether self-change towards trauma-centered identity in the aftermath of trauma is culturally specified. Therefore, it tests the prediction that centrality of the trauma memory can result in trauma-centered identity but such selfchange is dependent on self-consistency requirements. Study 1 found support for a culture distinction in self-change following trauma. Specifically, while the study replicated earlier findings (e.g., Sutherland \& Bryant, 2006) that stronger selfdefinition centered on trauma was positively related to severity of posttraumatic symptoms it was found that this was the case for Australians (independent culture) but not for Asians (interdependent culture). There was no relationship between traumacentered self-definition and posttraumatic symptomatology in the Asian group.

Study $3 c$ tests the prediction that self-concept dominated by the trauma event will differentiate PTSD sufferers and those without PTSD from an independent orientated culture but will not differentiate PTSD and non-PTSD trauma survivors from interdependent orientated cultures. These predictions are tested by asking trauma survivors from independent and interdependent cultures with and without PTSD to provide personal goals, self-defining memories and self-cognitions. Study $3 \mathrm{c}$ extends Study 1c by using three measures (goals, self-defining memories, self-cognitions) of personal identity as opposed to the one measure (self-defining memory task) that was able to be used in Study 1.

Study 3 also addresses the methodological limitations of the first series of studies by including measures that assess trauma history and independence/interdependence; including more culturally heterogeneous groups; having participants complete the data booklet at home reducing intercultural context effects; and requesting a significant life memory as opposed to a weekend memory and personal life goals as opposed to current goals in an attempt to replicate previous specificity, reflective comment and trauma-themed goal findings. 


\subsection{Hypotheses}

It is hypothesized that:

1/ replicating Study 1, the cultural distinction between autonomous versus relatedness orientation will be evident in everyday memories but not in trauma memories. Autobiographical memories of trauma for both independent and interdependent cultures will be predominately autonomous, (Study 3a)

$2 /$ the level of autonomous orientation will culturally differ in terms of differentiating between those with and without PTSD. Specifically, the interaction between culture and PTSD status for autonomous orientation will be significant. Those from independent cultures with PTSD will have less autonomous orientation in their trauma and everyday memories than those from independent culture without PTSD, whilst those from interdependent cultures with PTSD will have more autonomous orientation in their trauma and everyday memories when compared to those from interdependent cultures without PTSD (Study 3a),

3 / additionally, the interaction between culture and PTSD status will be significant in terms of autonomous orientation in the self-defining memories. Those from independent cultures with PTSD will have significantly less autonomous orientation in their self-defining memories than those from independent culture without PTSD. In contrast, those from interdependent cultures with PTSD will have significantly more autonomous orientation in their self-defining memories than those from interdependent culture without PTSD (Study 3b), and

4/ the interaction between PTSD status and culture will be significant for traumathemed personal goals, self-defining memories and self-cognitions. Specifically, those from independent cultures with PTSD will have significantly more trauma-themed goals, self-defining memories and self-cognitions than those from independent cultures without PTSD. However, those from interdependent cultures with PTSD will not describe a trauma-themed self-concept and hence, will not differ from those from interdependent cultures without PTSD, regarding trauma-centered goals, selfdefinition and self-cognition (Study 3c). 


\subsection{Method}

\subsubsection{Participants}

All participants $(N=106)$ were recruited from the general community by posters in public places, advertisements in local and ethnic newspapers, Adult Migrant English Programs and contacts with ethnic organizations and communities, and organizations that provide treatment for trauma survivors. The researchers promoted the study in places where people with a high probability of having a DSMIV (1994) PTSD Criteria A trauma experience would be reached (i.e., refugee communities, victims of crime etc.). Criteria A requires that an individual be exposed to a potentially traumatic event in which "the person has experienced, witnessed, or been confronted with an event that involved actual or threatened death or serious injury ... to oneself or others" and "the person's response involved intense fear, helplessness, or horror" (DSM-IV, 1994, pp. 427-428). Notices called for those who had experienced a traumatic event and identified the study as researching trauma, memory and culture. Participants received a $\$ 20$ supermarket voucher for their participation.

\subsubsection{General Measures}

\section{Independence/interdependence.}

Hofstede and Hofstede's (2004) individualism dimension was used to allocate participants into either the independent culture or interdependent culture group (Appendix A). The individualism dimension was developed based on extensive crosscultural research. Individualism is the degree the society reinforces individual or collective achievement and interpersonal relationships. A high individualism ranking indicates that individuality, independence and autonomy are dominant within the society. A low individualism ranking indicates the society is more collectivistic and emphasizes close ties between individuals and relatedness (Hofstede \& Hofstede, 2004). Based on their research, Hofstede and Hofstede comprised a list of various societies and their associated individualism score.

Cultural independence/interdependence was measured using a frequently used measure of the construct of self; the Twenty Statements Test (TST; Kuhn \& McPartland, 1954). The TST is a simple and commonly used technique to assess one's sense of self or self-identification (Kuhn \& McPartland, 1954). The TST is a 
qualitative measure of the self that makes explicit how individuals mediate their social environment in different ways by indexing how self-related information is differentially organized across individuals (Kuhn \& McPartland, 1954). The TST simply asks respondents to provide 20 statements in response to the question "Who Am I?" Researchers have frequently used the TST to examine and control for cultural differences in the individual's sense of self (Bochner, 1994; Dhawan, Roseman, Naidu, Thapa, \& Rettek, 1995; Ma \& Schoeneman, 1997; Rhee, Uleman, Lee, \& Roman, 1995; Wang et al., 1998; Watkins \& Gerong, 1999). The 20 responses are coded into comparable categories of the independent-interdependent dichotomy. The instrument allows researchers to observe individuals' own self-conceptualization (in a free-response format) of their social relationships, role identities, and personal qualities. The TST has been found to have high interrater reliability, criterion validity tests have generally supported the TST and researchers have shown the TST to have a fair degree of test-retest reliability (correlation coefficients for various coding schemes ranged from .38 to .85 , with test-retest intervals ranging from 2 weeks to 3 months; Kuhn \& McPartland, 1954; Spitzer, Couch, \& Stratton, 1973), content validity (Kuhn \& McPartland, 1954), and comparability with other self-instruments (in terms of concurrent validity; Spitzer et al.,1973).

\section{Posttraumatic stress disorder status.}

Posttraumatic stress disorder was diagnosed using the Posttraumatic Stress Diagnostic Scale (PDS; Foa, Riggs, Dancu, \& Rothbaum, 1993). The PDS was developed to provide a brief self-report instrument to assist with the diagnosis of PTSD. The PDS has four parts. Parts I and II contains trauma screening questions which correspond to DSM-IV (1994) PTSD Criteria A. Part III contains 17 items each corresponding to the Criteria B through Criteria D. Participants are asked to rate these items, for the past month, on a 4-point scale ranging from 0 (not at all) to 3 (almost always). A symptom is considered to be present if it is scored 1 or higher. The ratings of the items are summed to calculate a total severity score. The PDS then ascertains duration of the symptoms (Criteria E) and impairment of functioning (Criteria F). To be considered a positive screen on the PDS, a participant must meet Criteria A, endorse a broad enough range of items to meet Criteria B (reexperiencing), C (avoidance), and D (increased arousal), have symptoms present for over one month and indicate that the disturbances are causing significant impairment in functioning (i.e., a diagnosis of PTSD is only made if all the six DSM-IV criteria are endorsed. If 
one or more of the criteria is not met a diagnosis of PTSD is not made). In the initial validation study of the PDS, Cronbach's alpha was .91, test-retest reliability of the overall severity score was .74, and the PDS demonstrated concurrent and convergent validity with other measures of psychopathology (Foa et al., 1993). A subsequent investigation found the total-score Cronbach's alpha to be. 92 and test-retest reliability to be .83 (Foa, Cashman, Jaycox, \& Perry, 1997). Foa et al. (1993) examined the convergent validity of the PDS by comparing diagnoses based on the PDS to PTSD diagnoses based on the Structured Clinical Interview (SCID; Spitzer, Williams and Gibbon, 1987). The positive predictive power of the PDS was $100 \%$ and the negative predictive power was $82 \%$. Overall the PDS correctly identified the PTSD status of $86 \%$ of the subjects. $62 \%$ of the participants diagnosed with PTSD based on the SCID were also diagnosed with PTSD based on the PDS. The PDS correctly identified all participants who were not diagnosed with PTSD according to the SCID. The PDS has also been used in previous cross-cultural research with interdependent populations (e.g., Garcia, 2005).

To control for lifetime exposure to traumatic events, the Trauma History Questionnaire was used (THQ; Green, 1996). The THQ is designed to assess exposure to a wide range of potentially traumatic events, and was based on the "high magnitude stressor' questionnaire used in the DSM-IV (1994) field trials for PTSD (PTSD; Green, 1996). A range of traumatic events in three areas is assessed utilizing the THQ: namely, crime-related events (e.g., robbery, mugging), general disaster and trauma (e.g., injury, disaster, witnessing death), and unwanted physical and sexual experiences (Green, 1996). For each of the 24 items, the subject indicates whether or not he or she experienced the event, and if so, the number of times and approximate age(s) of occurrence. For the sexual and physical abuse questions, the subject is asked whether the experience was repeated, and if so, approximately how often and at what age. The questions in the THQ assess exposure to traumatic events included in the Criteria A1 of PTSD in the DSM-IV (1994). Test-retest reliability of the THQ has been found to be moderate to high and interrater reliability high (Mueser, et al., 2001). Furthermore, the THQ has also been tested in interdependent cultures and similar levels of referential meaning between the two back-translations and the original instrument were observed (e.g., Fiszman, Cabizuca, Lanfredi, \& Figueira, 2005). 


\section{Depression.}

Depression was measured using Part II of the Hopkins Symptom Checklist (HSCL-25; Derogatis et al., 1974) as outlined in Study 1.

\section{Demographics.}

Participants were asked to disclose their age, gender, length of time in Australia, ethnicity, religion and language spoken at home. Following this, participants were asked to rate on a 10-point scale from 1 (not at all) to 10 (extremely) how hard they found the tasks in the data booklet.

\subsubsection{Study 3a Measures}

\section{Autobiographical memory.}

Trauma event. Participants were asked to, "Please think about a significant, emotionally, traumatic event. Please write about this event in as much detail as you can. All your writing will be completely confidential. As you write do not worry about punctuation or grammar, just write as much as you can and you can include thoughts, feelings, reflections etc. If you need more room please use the next page." Following the narrative task, participants were asked to rate on a 10-point scale, from 1 (not at all) to 10 (extremely), how traumatic the event was and to indicate when the event occurred.

Everyday event. Participants were asked to, "Please think about an event that took place in your life. It may be positive, negative or neutral. Please write about the first event that comes to mind in as much detail as you can. All your writing will be completely confidential. As you write do not worry about punctuation or grammar, just write as much as you can and you can include thoughts, feelings, reflections etc. If you need more room, please use the next page." Participants were then asked to write down when the event occurred.

\subsubsection{Study $3 b$ Measures}

\section{Self-defining memories.}

Using Singer and Salovey's (1993) method, participants were informed that "A self-defining memory is a memory from your life that you remember very clearly, is important to you and leads to strong feelings, that may be either positive or negative, or both. It is the kind of memory that helps you to understand who you are and might be the memory you would tell someone else if you wanted that person to 
understand you in a more profound way. They are memories that you feel convey powerfully how you have come to be the person you currently are. Please briefly write down 5 self-defining memories."

\subsubsection{Study $3 c$ Measures}

\section{Self-concept measures.}

Goals. Following others researchers' (e.g., Emmons, 1986; Sutherland \& Bryant, 2006) measure of personal strivings, participants were instructed to "Please complete the following in the space provided. Please provide 15 goals that you feel are important for you to achieve".

Self-defining memories. As outlined above.

Self-cognitions. Given the TST has been used to examine self-definition in previous research (e.g., Bigner, 1971), the TST has been used in this study to examine trauma-themed self-definition in addition to independence/interdependence.

\subsubsection{Scoring/Coding System}

\section{Independence/interdependence.}

The TST was coded for independence and interdependence. Each participant's self-cognitions were coded as referring to independent (private) or interdependent (collective or public) aspects of self, in line with the definitions provided by Trafimow et al. (1991) and Triandis (1989). Therefore, self-cognitions were coded as independent (private) if the responses referred to personal qualities, attitudes, beliefs, or behaviors that were not related to other people (e.g., "I am kind", "I am happy"). Self-cognitions were coded as interdependent if they were collective self-cognitions (responses concerning to particular groups or categories, e.g., "I am Asian, woman, daughter") or cognitions pertaining to interdependence, friendship, and relationships or to the sensitivity of others (e.g., "I am in love"). Each participant received an independent and interdependent score, which was the ratio of each type of selfcognition divided by the number of cognitions provided.

\section{Study $3 a$}

The memory narratives were scored for theme, autonomous orientation, other/self ratio, interaction scenario, reflective comment and specificity as outlined in Study 1a. 


\section{Study $3 b$}

Content (theme, autonomous orientation, other/self and interaction scenario) and structure (elaboration and specificity) of self-defining memories were scored as outlined in Study 1b.

\section{Study $3 c$}

Three independent trauma-themed ratios were developed - goals, self-defining memories and self-cognitions. These ratios were formed by first coding each response as trauma-themed or not. To be coded as trauma-themed the response had to be directly and clearly related to trauma or survival; for goals ("Come to terms with loss of my baby", " Not be a freak covered in a burn scar", "Staying alive”, " Survive", "Recover", "Recovery", "Heal"), for self-defining memories (recalling a trauma event that is listed in Part 1 of the PDS' list of traumatic events; i.e., accident, natural disaster, non-sexual assault, sexual assault, military combat or war, child sexual abuse, imprisonment, torture or a life-threatening illness ) and for self-cognitions ("victim", "survivor, "resilient", "scared", "damaged", "a burns freak"). Second, the total number of trauma-themed responses was tallied for each participant. Then in order to control for possible differences in the number of responses provided on each measure, as not all participants provided the requested number of goals, memories and self-cognitions, adopting Jobson and O'Kearney's (2006) previous approach, the total number of trauma-themed responses were divided by the total number of goals, selfdefining memories and self-cognitions retrieved, respectively, to provide a traumatheme ratio for each measure.

\subsubsection{Design and Procedure}

Interested potential participants were sent a data package. Return rate was $42.4 \%$ (39.5\% interdependent, $45.3 \%$ independent). The data package contained a letter outlining the aims of the study, the instructions for participation and that if the participant decided to return the questionnaire they were giving their consent to having their questionnaire used in the study. The package also contained a reply paid self-addressed envelope so the participants could return the questionnaire to the researcher and a voucher slip. The voucher slip required participants to enter their name and address and these slips were returned with the questionnaire in the reply paid envelope. However, once the voucher was sent to the participant the slip was destroyed so the questionnaires were examined anonymously. Participants were 
informed of this in the letter. The questionnaire contained tasks pertaining to this study and Study 5. In the questionnaire, participants were asked to provide personal goals and self-defining memories, a trauma memory and everyday memory (counterbalanced) and to complete the questions pertaining to trauma memory meaning (Study 5) in one sitting. Participants were then asked to complete the PDS, HSCL-25, THQ, TST and demographics. Participants were instructed that these tasks need not be done in one sitting and could be done at participants own pace and time at home.

Participants were allocated to one of the two cultural groups based on their identified ethnicity. Participants' identified ethnicity was compared to Hofstede and Hofstede's (2004) categorization of cultures along the individualism/collectivism dimension (i.e., if the participant identified themselves as Australian they would be placed in the independent group whereas as a participant who identified themselves as Chinese would be placed in the interdependent group). Only cultures that could be clearly identified as individualistic or collectivistic were selected (i.e., cultures that are not distinctly individualistic or collectivistic were excluded). Two participants (both Spanish) were excluded based on this criterion. This allocation was then validated using the TST, as outlined in the results section.

Participants who met DSM-IV (1994) PTSD Criteria A on the PDS were allocated to either the PTSD or no PTSD group based on their completion of the remaining sections of the PDS. Nine participants (5 interdependent culture) were excluded as they did not meet Criteria A. Following PDS scoring, if participants endorsed items that were consistent with a DSM-IV diagnosis of PTSD (i.e., all six criteria are met) participants were allocated to the PTSD group.

\subsubsection{Reliability}

A second independent rater who was Chinese coded $20 \%$ of responses. Raters were blind to the cultural group and PTSD status of participants. The second rater was blind to study hypotheses. Discrepancies between raters were resolved through discussion. Interrater reliability was good for independence/interdependence (TST) $($ Kappa coefficient $=.89)$.

\section{Study $3 a$.}

For the narratives, raters had $87.5 \%$ agreement on theme $($ Kappa coefficient $=$ .67 ) and $95 \%$ agreement on specificity (Kappa coefficient $=.86$ ). The mean Kappa 
coefficient of reliability was .72 for autonomous orientation, .74 for interaction scenario and .70 for reflective comment.

\section{Study $3 b$.}

For the self-defining memories, the mean Kappa coefficient of reliability was .79 for theme, .73 for autonomous orientation, .73 for interaction scenario, .69 for elaboration and .82 for specificity.

\section{Study 3c.}

For trauma-themed self-concept, the mean Kappa coefficient of reliability was .89 for trauma-themed goals, .81 for trauma-themed self-defining memories and .94 for trauma-themed self-cognitions.

\subsection{Results}

\subsubsection{Group Characteristics}

Group characteristics of independence/interdependence, PTSD severity and trauma exposure are outlined in Table 7.1.

Table 7.1

Means and (Standard Deviations) of Group Characteristics

\begin{tabular}{lcccc}
\hline & \multicolumn{2}{c}{ Independent culture } & \multicolumn{2}{c}{ Interdependent culture } \\
& PTSD & No PTSD & PTSD & No PTSD \\
\hline TST & & & & \\
Independent & $.75(.27)$ & $.64(.25)$ & $.52(.27)$ & $.45(.27)$ \\
\multicolumn{1}{c}{ Interdependent } & $.25(.27)$ & $.36(.25)$ & $.48(.27)$ & $.55(.27)$ \\
\hline PDS total score & $26.80(8.63)$ & $3.06(4.52)$ & $22.25(11.11)$ & $3.76(4.60)$ \\
\hline THQ & & & & \\
Crime-related & $1.83(1.75)$ & $1.13(1.26)$ & $1.17(2.14)$ & $1.92(2.69)$ \\
General Disaster & $4.40(2.09)$ & $3.26(2.74)$ & $3.83(2.60)$ & $3.72(2.79)$ \\
Physical/Sexual & $1.53(1.48)$ & $.68(1.11)$ & $.83(1.74)$ & $.64(1.35)$ \\
\hline
\end{tabular}




\section{Independence/interdependence.}

Using Hofstede and Hofstede's (2004) cultural allocation of individualism scores, the independent $(M=87.05, S D=11.23)$ group was significantly higher on the individualism dimension than the interdependent group, $(M=26.22, S D=14.15)$, $t(104)=24.67, p<.01$. Supporting this, the independent group provided significantly more independent and less interdependent statements on the TST than the interdependent group, $t(104)=3.89, p<.01$.

\section{PTSD severity and trauma exposure.}

All participants were trauma-exposed. There were no cultural differences in terms of PTSD symptom score on the PDS, $F(1,102)=1.71, p=.20$. Those with PTSD scored significantly higher than those without PTSD, $F(1,102)=204.91, p<$ .01 . The interaction was not significant, $F(1,102)=3.16, p=.08$. Given the near significance of this finding, the interaction was followed up with $t$ tests to ensure the groups were matched in terms of PTSD symptomatology. The independent PTSD group was significantly higher in terms of level of PTSD symptomatology than the independent no PTSD group, $t(55)=13.31, p<.01$. The interdependent PTSD group was significantly higher in terms of level of PTSD symptomatology than the interdependent no PTSD group, $t(47)=7.67, p<.01$. The independent PTSD and interdependent PTSD groups did not differ in terms of level of PTSD symptomatology, $t(48)=1.62, p=.11$, nor did the independent no PTSD and interdependent no PTSD groups, $t(54)=.57, p=.57$. Hence, it was assumed the groups were equally matched.

In terms of the distribution of trauma types as indicated on the PDS, the independent group ( 8 crime-related, 30 general disaster and trauma, 19 unwanted physical and sexual experiences) did not differ from the interdependent group (7 crime-related, 27 general disaster and trauma, 15 unwanted physical and sexual experience), $\chi^{2}(2, N=106)=.09, p=.96$. The independent group ( 3 less than six months ago, 23 six months to five years ago, 31 more than five years ago) and interdependent group ( 8 less than six months ago, 18 six months to five years ago, 23 more than five years ago) did not differ in terms of length of time since the traumatic event happened as indicated on the PDS, $\chi^{2}(2, N=106)=3.48, p=.18$. The independent and interdependent group were equivalent in terms of trauma history (THQ); crime-related event, $t(104)=.27, p=.79$, general disaster and trauma, $t(104)$ $=.00, p>.99$, and unwanted physical and sexual experiences, $t(104)=1.19, p=.24$. 
In terms of trauma distribution, as indicated on the PDS, the PTSD group (6 crime-related, 25 general disaster and trauma, 19 unwanted physical and sexual experiences) did not differ from the no PTSD group (9 crime-related, 32 general disaster and trauma, 15 unwanted physical and sexual experiences), $\chi^{2}(2, N=106)=$ $1.60, p=.45$. The PTSD group (6 less than six months ago, 22 six months to three years ago, 22 more than five years ago) and no PTSD group (5 less than six months ago, 19 six months to three years ago, 32 more than five years ago) did not differ in terms of length of time since the traumatic event happened as indicated on the PDS, $\chi^{2}$ $(2, N=106)=1.83, p=.40$. The PTSD and no PTSD groups were equivalent in terms of trauma history (THQ); crime-related event, $t(104)=.07, p=.94$, general disaster, $t$ $(104)=1.33, p=.19$, and unwanted physical and sexual experiences, $t(104)=1.94, p$ $=.06$.

\subsubsection{Participant Characteristics}

The independent PTSD group (6 male, 20 female) ranged in age from 20 to 60 years $(M=41.15, S D=12.30)$ and had lived in Australia for .20 to 60 years $(M=$ $36.78, S D=14.48)$. The ethnic distribution was $69.23 \%$ Australian/New Zealander, 26.92\% Western European and 3.85\% American. The entire sample reported that they spoke English at home and religious affiliation distribution was $46.15 \%$ no religious affiliation, $46.15 \%$ Christian/Catholic, 3.85\% Buddhist and 3.85\% Yogi.

The independent no PTSD group (6 male, 25 female) ranged in age from 19 to 60 years $(M=40.16, S D=14.12)$ and had lived in Australia for 6.50 to 60 years $(M=$ 34.94, $S D=15.49$ ). The ethnic distribution was $83.87 \%$ Australian/New Zealander, $12.90 \%$ Western European and $3.22 \%$ American. Language spoken at home distribution was $96.77 \%$ English and 3.22\% French. Religious affiliation distribution was $80.65 \%$ Christian/Catholic and $19.35 \%$ no religious affiliation.

The interdependent PTSD group ( 9 male, 15 female) ranged in age from 20 to 60 years $(M=34.25, S D=14.18)$ and had lived in Australia for .25 to 37 years $(M=$ $5.49, S D=8.95)$. The ethnic distribution was $20.83 \%$ Southeast Asian, $20.83 \%$ African, 20.83\% Asian, 20.83\% Middle Eastern and 16.67\% Eastern European. Language spoken at home distribution was $16.67 \%$ Chinese, $16.67 \%$ English, $12.5 \%$ Krio, $8.33 \%$ Russian, $8.33 \%$ Hindi and $4.17 \%$ for each of the following languages Indonesian, Telugu, French, Dinka, Urdu, Khmer, Thai, Korean and Arabic. Religious 
affiliation distribution was $33.33 \%$ Christian/Catholic, $25 \%$ Islamic, $16.67 \%$ no religious affiliation, $12.50 \%$ Hindu and $12.50 \%$ Buddhist.

The interdependent no PTSD group (11 male, 14 female) ranged in age from 21 to 60 years $(M=33.24, S D=12.91)$ and had lived in Australia for 10 to 37 years $(M=7.22, S D=12.91)$. The ethnic distribution was $36.00 \%$ Asian, $28.00 \%$ Southeast Asian, $12.00 \%$ African, $12.00 \%$ Middle Eastern, $8.00 \%$ Eastern European and $4.00 \%$ South American. Language spoken at home distribution was $32.00 \%$ English, $16.00 \%$ Chinese, $12.00 \%$ Japanese, $8.00 \%$ Indonesian, $8.00 \%$ Mon and $4.00 \%$ for each of the following languages Malayalam, Farsi, Hindi, Dinka, Portuguese and Kirundi. Religious affiliation distribution was $28.00 \%$ no religious affiliation, $20.00 \%$ Buddhist, $20.00 \%$ Christian/Catholic, $16.00 \%$ Islamic, $12.00 \%$ Hindu and $4.00 \%$ New Age.

The independent group was significantly older than the interdependent group, $F(1,102)=6.96, p=.01$. Age will be used as a covariate throughout. The PTSD and no PTSD groups did not differ in terms of age, $F(1,102)=.15, p=.70$, and the interaction was not significant, $F(1,102)=.00, p>.99$. The four groups did not differ in terms of gender distribution, $\chi^{2}(3, N=106)=5.97, p=.11$. Those from interdependent cultures had lived in Australia for significantly less time than those from independent cultures, $F(1,102)=144.04, p<.01$. However, there was no significant difference between those with and without PTSD regarding length of time in Australia, $F(1,102)=.00, p=.98$, and the interaction was not significant, $F(1$, $102)=.53, p=.47$. There were no cultural differences $(M=4.93, S D=2.78$ independent; $M=5.21, S D=2.66$ interdependent) in reports of how hard participants found the study, $F(1,102)=.26, p=.61$, nor for PTSD status differences $(M=5.49$, $S D=2.88$ PTSD $M=4.65, S D=2.56$ no PTSD $), F(1,102)=2.45, p=.12$. The interaction was not significant, $F(1,102)=1.25, p=.27$.

The independent culture group $(M=1.86, S D=.52)$ did not differ from the interdependent culture group $(M=1.82, S D=.45)$ in terms of depression, $F(1,102)=$ $.10, p=.75$. However, those with PTSD $(M=2.70, S D=.55)$ were significantly more depressed than those without PTSD $(M=1.41, S D=.42), F(1,102)=81.35, p<.01$. Depression will be used as a covariate throughout. The interaction was not significant, $F(1,102)=2.41, p=.12$. 


\subsection{Study 3a}

The hypotheses tested in Study $3 \mathrm{a}$ are that:

$1 /$ replicating Study 1 , the cultural distinction between autonomous versus relatedness orientation will be evident in everyday memories but not in trauma memories. Autobiographical memories of trauma for both independent and interdependent cultures will be predominately autonomous, and

$2 /$ the level of autonomous orientation will culturally differ in terms of differentiating between those with and without PTSD. Those from independent cultures with PTSD will have less autonomous orientation in their trauma and everyday memories than those from independent culture without PTSD, while those from interdependent cultures with PTSD will have more autonomous orientation in their trauma and everyday memories when compared to those from interdependent cultures without PTSD.

\subsubsection{Results}

\section{Preliminary analysis for narrative task.}

Narrative type. The trauma narratives were classified into the following trauma type categories; death $(n=29)$, relationship loss/affair/family problems $(n=$ 16), non-sexual assault (including domestic violence) $(n=12)$, child abuse $(n=10)$, serious physical injury, illness $(n=8)$, war (including refugee experience) $(n=8)$, natural disaster $(n=6)$, sexual assault $(n=5)$, serious accident (e.g., motor vehicle accident, lost in bush) $(n=6)$, and torture/kidnapped $(n=6)$. The four groups did not

differ in terms of trauma type distribution, $\chi^{2}(27, N=106)=39.43, p=.06$. Given the near significance of this finding, trauma narrative type analyses were followed up further. It was found that all groups were relatively similar on all trauma types except war and torture. Those from interdependent cultures seemed to recall more war memories than those from independent cultures.

The everyday narratives were classified as family interaction $(n=35)$, achievement (e.g., travel, migration, buying house, academic, employment) $(n=32)$, relationship $(n=20)$ and accident/war/physical injury/illness/assault $(n=19)$. The four groups did not differ in terms of everyday type distribution, $\chi^{2}(9, N=106)=$ $10.74, p=.29$. The everyday memories were classified as either trauma or nontrauma. To be classified as trauma, the memory had to recall an event type listed in 
Part 1 of the PDS' list of traumatic events (i.e., accident, natural disaster, non-sexual assault, sexual assault, military combat or war, child sexual abuse, imprisonment, torture or a life threatening illness). The cultural groups (7.01\% of independent culture and $12.24 \%$ of interdependent culture retrieved a trauma memory) did not differ in terms of everyday memory being classified as a trauma memory, $\chi^{2}(1, N=$ $106)=.84, p=.36$. There was also no difference between PTSD status groups (12.00\% of the PTSD group and $7.14 \%$ of the no PTSD group retrieved a trauma memory), $\chi^{2}(1, N=106)=.73, p=.39$.

Self-rated trauma severity. There was no significant difference between the cultural groups' $(M=8.89, S D=1.83$ independent; $M=8.77, S D=1.85$ interdependent) reports of how traumatic they found the trauma event, $F(1,102)=$ $.08, p=.77$, nor any differences between those with $(M=8.92, S D=1.87)$ and without PTSD $(M=8.73, S D=1.81), F(1,102)=.28, p=.60$. The interaction was not significant, $F(1,102)=.04, p=.83$.

Self-rated time of narrative event. There was no significant difference between the independent culture's $(M=6.85$ years, $S D=3.33$ years trauma; $M=5.93$ years, $S D=3.65$ years everyday) and interdependent culture's $(M=6.44$ years, $S D=$ 3.42 years trauma; $M=6.58$ years, $S D=3.69$ years everyday) self-reports of when the trauma event, $F(1,102)=.40, p=.53$, and everyday event, $F(1,102)=.82, p=.44$, occurred. Those with PTSD $(M=6.09$ years, $S D=3.68$ years trauma; $M=6.15$ years, $S D=3.63$ years everyday $)$ and those without PTSD $(M=7.20$ years, $S D=3.08$ years trauma; $M=6.33$ years, $S D=3.71$ years everyday), did not differ in terms of when the trauma event, $F(1,102)=2.87, p=.09$, or everyday event, $F(1,102)=.08, p=$ .77 , occurred. The interactions were not significant, $F(1,102)=.07, p=.80$ (trauma), $F(1,102)=1.00, p=.32$ (everyday)

Fluency. A 2 × 2 × 2 way ANOVA with culture (interdependent vs. independent) and PTSD status (PTSD vs. no-PTSD) as between subject factors and memory type (everyday vs. trauma) as a within subject factor with number of words as the dependent measure was conducted. Fluency of trauma memories $(M=169.08$, $S D=122.23)$ was significantly greater than everyday memories $(M=125.49, S D=$ 77.26), $F(1,102)=20.03, p<.01$. There were no differences in fluency in independent $(M=158.77, S D=105.01)$ versus interdependent cultures $(M=133.94$, $S D=94.51)$ overall, $F(1,102)=1.93, p=.17$, and the interaction between culture and memory type was not significant, $F(1,102)=.41, p=.53$. There were no 
differences in fluency in PTSD $(M=141.19, S D=102.33)$ versus no PTSD $(M=$ $152.73, S D=97.57), F(1,102)=.38, p=.54$, and no interaction between PTSD status and memory type, $F(1,102)=1.53, p=.22$. The interaction between culture and PTSD status was not significant, $F(1,101)=.01, p=.94$, nor the three-way interaction, $F(1,102)=.43, p=.52$.

\section{Comparative analysis of narratives.}

For the dichotomous narrative measures (theme and specificity) chi-square statistical analyses were conducted. To explore culturally dependent differences in the everyday memories and trauma memories, a chi-square using culture (independent; interdependent) and either theme (autonomous; relatedness) or specificity (specific; general) was used. To examine PTSD status differences in the everyday memories and trauma memories, a chi-square with PTSD status (PTSD; no PTSD) and theme (autonomous; relatedness) and specificity (specific; general) was used.

$2 \times 2 \times 2$ way ANCOVAs with culture (independent vs. interdependent) and PTSD status (PTSD vs. no PTSD) as between subject factors, memory type (everyday vs. trauma) as a within subject factor, with age and depression as covariates and the narrative measures as the dependent variables were conducted. Following Study 1, significant culture by memory type interactions were followed up using both paired and independent $t$ tests with the adjusted means. Independent group $t$ tests were used to compare cultural differences in the trauma and everyday memories. Paired $t$ tests were used to compare memory type differences. Interactions between culture and PTSD status were followed up using independent group $t$ tests; independent culture with PTSD versus independent culture without PTSD and interdependent culture with PTSD versus interdependent culture without PTSD.

Theme. For the everyday memory the two cultural groups did not differ in terms of memory theme distribution, $\chi^{2}(1, N=106)=1.18, p=.28$. However, the trend was in the expected direction. The independent group tended to provide fewer relatedness-themed memories $(61.40 \%)$ and more autonomous-themed memories $(38.60 \%)$ than the interdependent group $(71.43 \%, 28.57 \%)$. The PTSD status groups did not differ in terms of everyday memory theme distribution, $\chi^{2}(1, N=106)=1.54$, $p=.22$, $(60.00 \%$ of the PTSD group and $71.43 \%$ of the no PTSD group provided a relatedness everyday memory).

For the trauma memory, the cultural groups did not differ in terms of memory theme distribution, $\chi^{2}(1, N=106)=.34, p=.56$. Independent cultures were as likely 
to provide autonomous themed trauma memories $(33.33 \%)$ and relatedness themed trauma memories $(66.67 \%)$ as their interdependent counterparts $(38.88 \%, 61.22 \%)$. Those with PTSD were as likely to provide as many autonomous themed trauma memories $(38.00 \%)$ and relatedness themed memories $(62.00 \%)$ as those without $\operatorname{PTSD}(33.93 \%, 66.07 \%), \chi^{2}(1, N=106)=.19, p=.66$.

Autonomous orientation. Figure 7.2 shows the adjusted means for autonomous orientation. Two participant's everyday memory data was excluded as outliers (1 from independent no PTSD and 1 from interdependent PTSD).

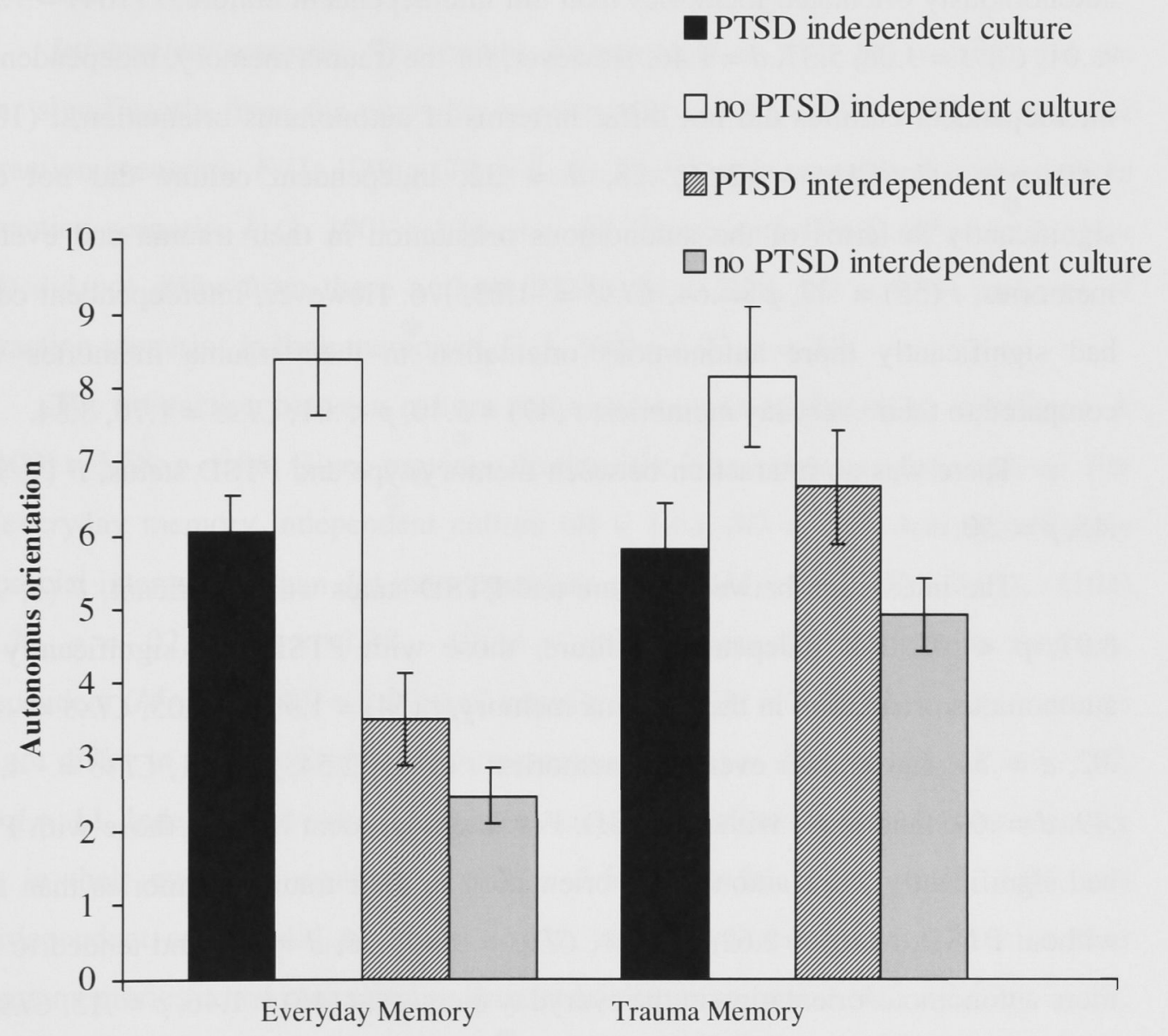

Figure 7.2 The adjusted mean autonomous orientation for the independent PTSD group, independent no PTSD group, interdependent PTSD group and interdependent no PTSD groups for both the everyday and trauma memory. Error bars indicate \pm 1 SE of the mean. 
Overall, the trauma memory did not differ significantly from the everyday memory in terms of autonomous orientation, $F(1,98)=.59, p=.44$. Interdependent cultures had significantly less autonomous orientation in their memories than did independent cultures, $F(1,98)=18.20, p<.01$. There was no significant difference in autonomous orientation between those with and without PTSD, $F(1,98)=.00, p=$ .97.

The interaction between culture and memory type was significant, $F(1,98)=$ $17.41, p<.01$. For the everyday memory, independent culture had significantly more autonomously orientated memories than did interdependent culture, $t(104)=7.33, p$ $<.01, C I .95=3.08,5.37, d=1.46$. However, for the trauma memory, independent and interdependent cultures did not differ in terms of autonomous orientation, $t(102)=$ $1.60, p=.11, C 1.95=-2.66, .28, d=.32$. Independent culture did not differ significantly in terms of the autonomous orientation in their trauma and everyday memories, $t(55)=.47, p=.64, C 1.95=-1.23, .76$. However, interdependent culture had significantly more autonomous orientation in their trauma memories when compared to their everyday memories, $t(47)=5.40, p<.01, C I .95=1.76,3.84$.

There was no interaction between memory type and PTSD status, $F(1,98)=$ $.45, p=.50$.

The interaction between culture and PTSD status was significant, $F(1,98)=$ 8.97, $p<.01$. For independent culture, those with PTSD had significantly less autonomous orientation in their trauma memory, $t(54)=1.99, p=.05, C I .95=-4.74$, $.02, d=.54$, and in their everyday memories, $t(54)=2.54, p<.01, C I .95=-4.19$, $.49, d=.69$, than those without PTSD. For interdependent culture, those with PTSD had significantly more autonomous orientation in their trauma memories than those without PTSD, $t(46)=2.62, p=.01, C I .95=.68,5.16, d=.55$, and tended to have more autonomous orientation in the everyday memory, $t(46)=1.46, p=.15, C I .95=-$ $.39,2.49, d=.42$.

There was no interaction between memory type, culture and PTSD status, $F$ $(1,98)=.17, p=.68$.

Other/self ratio. Five participant's (3 independent no PTSD, 1 independent PTSD, 1 interdependent no PTSD) other/self ratios for the trauma memory had to be excluded as outliers. Four participant's (2 independent no PTSD, 1 interdependent PTSD, 1 interdependent no PTSD) other/self ratios for the everyday memory had to be excluded as outliers. There was no significant difference in other/self ratio in the 
trauma memories $(M=.75, S D=.50)$ when compared to the everyday memories $(M=$ $.75, S D=.60), F(1,91)=.00, p=.98$. Independent culture $(M=.72, S D=.47) \mathrm{did}$ not differ significantly from interdependent culture $(M=.78, S D=.63), F(1,91)=$ $.49, p=.49$. There was also no significant difference in other/self ratio between those with $(M=.71, S D=.55)$ and without PTSD $(M=.79, S D=.55), F(1,91)=.73, p=$ .39. The interactions between culture and memory type, $F(1,91)=1.07, p=.30$, PTSD status and memory type, $F(1,91)=.20, p=.66$, and culture and PTSD status, $F(1,91)=.71, p=.40$, were not significant. The three-way interaction was not significant, $F(1,91)=.06, p=.81$.

Interaction scenario. The trauma memory $(M=2.14, S D=1.76)$ did not differ significantly from the everyday memory $(M=1.97, S D=1.68)$ in terms of interaction scenarios, $F(1,100)=.70, p=.40$. There were no cultural differences in interaction scenario, $F(1,100)=1.18, p=.28$. Those with PTSD $(M=1.82, S D=$ 1.68) did not differ from those without PTSD $(M=2.28, S D=1.74)$ in terms of interaction scenarios in their memories, $F(1,100)=1.73, p=.19$.

The interaction between culture and memory type tended to be significant, $F$ $(1,100)=3.58, p=.06$. Given previous findings the interaction was followed-up. For the everyday memory independent culture $(M=1.63, S D=1.26)$ had significantly less social interactions than did interdependent culture $(M=2.37, S D=2.01), t(104)$ $=2.30, p=.02$, CI.95 $=-1.38,-.10, d=.44$, however, for the trauma memory, independent $(M=2.23, S D=1.59)$ and interdependent $(M=2.20, S D=2.16)$ cultures did not differ in terms of interaction scenarios, $t(104)=.55, p=.58, C I .95=-.87$, $.49, d=.11$. Independent culture had more social interactions in their trauma memory than in their everyday memory, $t(56)=2.68, p=.01, C I .95=.15,1.04$, and interdependent culture did not differ in the number of social interactions in their trauma memories when compared to their everyday memories, $t(48)=1.09, p=.28$, $C I .95=-.93, .28$.

The interactions between culture and PTSD status was not significant, $F$ (1, $100)=.05, p=.83$, or between memory type and PTSD status, $F(1,100)=.05, p=$ .83 , or between memory type, culture and PTSD status, $F(1,100)=.09, p=.77$.

Reflective comment. Figure 7.3 shows the mean reflective comment. Two participant's ( 1 independent PTSD, 1 independent no PTSD) reflective comment scores for the trauma memory and two participant's (2 independent no PTSD) reflective comment scores for the everyday memory had to be excluded as outliers. 
PTSD independent culture

$\square$ no PTSD independent culture

WTSD interdependent culture

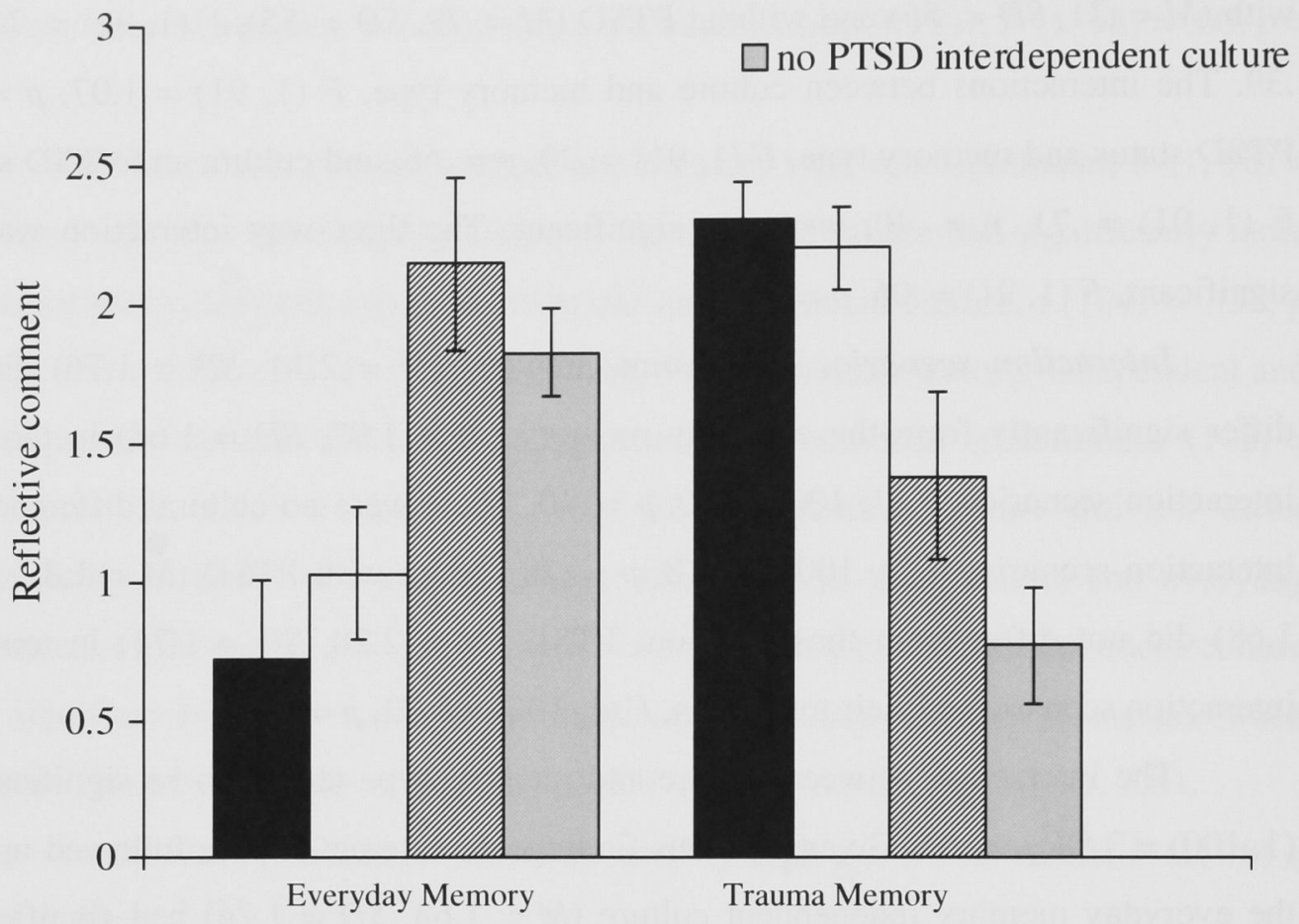

Figure 7.3 The adjusted mean reflective comments for the independent PTSD group, independent no PTSD group, interdependent PTSD group and interdependent no PTSD groups for both the everyday and trauma memory. Error bars indicate \pm 1 SE of the mean.

The trauma memory did not differ significantly from the everyday memory in terms of reflective comment, $F(1,97)=.04, p=.84$. There were no cultural differences in reflective comment, $F(1,97)=1.95, p=.17$. Those with PTSD had significantly more reflective comments than those without PTSD, $F(1,97)=5.02, p$ $=.03$.

The interaction between culture and memory type was significant, $F(1,97)=$ $48.80, p<.01$. For the everyday memory interdependent culture had significantly more reflective comments than did independent culture, $t(101)=6.07, p<.01, C I .95$ $=.83,1.65, d=1.18$. However, for the trauma memory, independent culture had significantly more reflective comments than did interdependent culture, $t(101)=$ 
$2.96, p<.01, C I .95=.25,1.27, d=.59$. Independent culture had significantly more reflective comments in their trauma memory than in their everyday memories, $t(53)=$ $5.99, p<.01, C I .95=.72,1.44$. However, interdependent culture had significantly more reflective comments in their everyday memories when compared to their trauma memories, $t(48)=4.61, p<.01, C I .95=.52,1.32$.

The interactions between culture and PTSD status, $F(1,97)=1.25, p=.27$, memory type and PTSD status, $F(1,97)=.07, p=.79$, and memory type, culture and PTSD status, $F(1,97)=.23, p=.63$, were not significant.

Memory specificity. When compared to the interdependent group, (40.82\%, $38.78 \%$ respectively), the independent group $(70.18 \%, 78.95 \%$, respectively) provided more specific everyday memories, $\chi^{2}(1, N=106)=9.25, p<.01$, and trauma memories, $\chi^{2}(1, N=106)=17.77, p<.01$. Those with PTSD $(50.00 \%$, $60.00 \%$, respectively) did not differ from those without PTSD $(62.50 \%, 60.71 \%$, respectively) in specificity of everyday memories, $\chi^{2}(1, N=106)=1.68, p=.20$, or trauma memories, $\chi^{2}(1, N=106)=.01, p=.94$.

Furthermore, it was found that for the independent group those with PTSD $(50.00 \%)$ provided significantly less specific everyday memories than those without PTSD $(87.10 \%), \chi^{2}(1, N=57)=9.30, p<.01$, but did not differ $(69.23 \%$ PTSD, $87.10 \%$ no PTSD) in terms of trauma memory specificity distribution, $\chi^{2}(1, N=57)=$ $2.72, p=.10$. This finding was evident even when depression was controlled for. However, interdependent culture with $(50.00 \%, 50.00 \%)$ and without $(32.00 \%$, $28.00 \%)$ PTSD did not differ in specificity distribution for either the everyday memory, $\chi^{2}(1, N=49)=1.64, p=.20$, or the trauma memory, $\chi^{2}(1, N=49)=2.50, p$ $=.11$.

\subsubsection{Discussion}

Overall, the results support the findings of Study 1 and hence, further confirmed expectations derived from the conceptual framework. Namely, those from independent cultures tended to provide less relatedness-themed everyday memories and more autonomous-themed everyday memories than the interdependent group. However, for the trauma memory, the cultural groups did not differ in terms of memory theme. Greatest support for the hypothesis comes from the finding that those from independent cultures had significantly more autonomously orientated everyday memories than did those from interdependent cultures. However, for the trauma 
memory, independent and interdependent cultures did not differ in terms of autonomous orientation. Additionally, while those from independent cultures did not differ in terms of the autonomous orientation in their trauma and everyday memories, those from interdependent cultures had significantly more autonomous orientation in their trauma memories when compared to their everyday memories. Unexpectedly, the cultural groups did not differ in terms of egocentricity (other/self ratio) of either their everyday or trauma memories. Finally, those from independent cultures had significantly less social interactions in their everyday memories than did those from interdependent culture, while those from independent and interdependent cultures did not differ in terms of the number of social interactions in the trauma memory. However, this was the result of those from independent cultures having more social interactions in their trauma memory than in their everyday memory rather than the expected finding that those from interdependent cultures would have less social interactions in their trauma memories when compared to their everyday memories.

It is believed that of the memory variables (theme, autonomous orientation, other/self ratio and interaction scenarios), autonomous orientation is the clearest measure of autonomy in autobiographical remembering, and thus the activation of an autonomous goal hierarchy (working self), as autonomous orientation is a measure of a person's "tendency to express autonomy", "personal evaluations", personal control and "self-determination" (Wang \& Conway, 2004, p. 920). In contrast, theme refers to the subject matter of the autobiographical memory and other/self ratio and interaction scenario are measures of "social orientation" (Wang \& Conway, 2004, p. 920). Therefore, findings regarding levels of autonomous orientation in the trauma memory provide strong support for the first hypothesis. Thus, there is evidence that trauma by its nature primes an autonomous working self, regardless of the culturally dominant self. Therefore, the cultural distinction between autonomy versus relatedness evident for autobiographical remembering seems not to be salient in autobiographical remembering of trauma. The remembering of a traumatic event seems to be predominantly autonomous.

As evident in the following selected everyday memories that resemble the typical response of each cultural group, Study 3 a found that for the autobiographical remembering of everyday events those from independent culture tended to provide more autonomous-themed, more autonomously-oriented everyday memories with less social interactions than those from interdependent culture. 
An event that took place in my life that was very beneficial and positive for myself was travelling overseas by myself. I loved the experience, I loved being free, making my own decisions, going anywhere I pleased and seeing and meeting new people. I believe the experience grew me as a person and I learnt a lot about myself and people around me. My feelings as I left the airport were those of worry, sadness and apprehension of the unknown. But as the trip progressed I felt more at ease, more excited and started to feel more comfortable with myself and my surroundings. Finally, as I prepared to come home I had a mixture of feelings...Looking back on my time overseas I know it was beneficial for me as it help put things in perspective as well as learn a lot about myself and what the world has to offer. The trip has also allowed me to work out my goals...Overall, the trip was very positive in regard to understanding myself and my life at the present and in the future.

- Independent no PTSD group

The scene: presentation day for the soccer club. Sunny pleasant day. BBQ. Lots of families. Mid 70's. I was 14-15 years old. My focus in life was soccer. I loved it! The team I played in was a B-grade team - the top grade for my age group. We made the finals but didn't win the comp[etition]. When the team awards were given out, I received the "most improved" - most unexpectedly. I remember being/feeling overwhelmed, proud, lost for words. I sat on the ground while everyone in the team was still standing. I wanted to cry and fought back the tears. There were players on the team who were much more skilful than I and I wanted to play like them. I didn't want to be like them as people - I didn't respect their character or lifestyle; I just wanted to be as good or better than them at soccer. They were congratulating me and praising the way I had played. I could tell they were genuine. As I reflect on this I think I was also feeling confused and bewildered since there was a contradiction in their behaviour. I was believing what they were saying that I deserved the award but it was coming from the place I least expected.

- Independent no PTSD group

I was born in a family that had some problems. My father had quarrels with his brother and his family. We were neighbours in the same street. My mother 
and my sisters were scared from them. They tried to break into our home and fight with us about low value things that we shared with them (like our garden and its products, money...) until my aunty died (after 10 years) we still had a quarrel for 5 years until my uncle (my father's brother) died. In that time my family was innocent. We had no choice and could not move out of our home because my father still liked living close to them and having relations with them (my father was a good father for us).

- Interdependent no PTSD group

A love story. It was about a girl who was my girlfriend in refugee camp. She told me she was in love with me and that my time with her completely changed her life and I had changed her from a men-shy girl to what she turned out to be, a lover. She also told me she was a virgin a thing highly valued in our community. This makes us stay without indulging in any intercourse which was a great disappointment from my friends who had played sex with their girlfriends making me feel left out of the group.

\section{- Interdependent PTSD group}

As evident in the following selected trauma memories that resemble the typical response of each cultural group, for the autobiographical remembering of trauma, those from independent and interdependent culture did not differ in terms of theme, number of social interactions or autonomous orientation. The independent group remained as autonomously oriented in their trauma memory as in their everyday memory, while the interdependent group was significantly more autonomously oriented in their trauma memory when compared to their everyday memory.

When I was 17, a friend took me out water-skiing. He gave me the second half of his beer to drink. Later I started to feel drunk and I got more and more drowsy. I remember sitting on the passenger seat of the car, white sedan...He took the car up to the top of the mountain overlooking the city. We got out of the car but I could barely stand up. He pushed me against the car and tried kissing me and then pulled me back on the passenger seat and got on top of me. I don't really remember the sequence of things but later on I realised he 
was penetrating me and asked him to stop, but he kept going and I kept begging him to stop. I was crying. He stopped when he wanted.

- Independent PTSD group

When I was swimming in a swimming pool there was a man who disturbed me. He was looking for me and touched my legs. I was very afraid.

- Interdependent no PTSD group

29 Feb 2006 will be a day that I will remember for a long time, mainly because it was the first time I saw someone die. I was travelling as usual to work when the car in front of me ran off the road and into the side barrier which then bounced back onto the road running into another vehicle. Both vehicles veered off the road - the passenger in the second car died on the spot the other driver was knocked unconscious and had to be taken to hospital. That day was the first day in my life that I thought I could have died - that car could have been me if I was 30 seconds earlier or if I sped that would have been me - it would have been me that people were trying to help. It has been the first time in my life where death has confronted me and played a role in my life...I reflect and become aware that my life could end at any time - I guess this event has taught me something in life.

\section{- Independent no PTSD group}

I had three bad experiences...road accidents. The first and second accidents occurred where I was hit by another motorcycle when I was riding my motorcycle. I didn't know the accident had occurred for the first and second times, I had just realised I had been in an accident when I was aware that I was lying on the floor and I felt pain. I was not that hurt (physically). Then I had my third road accident, but that was the worst experience in my life. During the $31^{\text {st }}$ December 2004 , I drove my car on my way to return to my hometown. The journey normally took me around 2 hours. That was a raining day. My mood was quite down at that moment. I didn't drive so fast, just around $60 \mathrm{~km} / \mathrm{h}$ because there was a truck in front of my car that I followed it for nearly 20 minutes already. At one stage, I found that there was no car from the other side of the road, so I sped up my car and tried to overtake the truck. I had 
success but I found that a car was coming towards my car. I turned the steering wheel to the right to go back to my track but unfortunately there is a steepen slope and I found that I couldn't control my car anymore. I was on my seat and looking at my car hit the truck. I didn't scream because my mind was getting blank. I saw by myself that my car hit the truck. I even had no time to feel worry or scared. I was watching the whole process of my car hit the truck. My car was gone but I was safe. Compared with my past experiences I was not that hurt but it took me a long time to recover (my emotions) from this traumatic event.

\section{- Interdependent no PTSD group}

Regarding the second hypothesis, as predicted those from independent culture with PTSD had less autonomous orientation in their trauma and everyday memories than those without PTSD. In contrast, those with PTSD from interdependent culture had more autonomous orientation in their trauma memory and tended to have more autonomous orientation in the everyday memory than those without PTSD. This finding supports the further developments made to the conceptual framework outlined in the introduction.

Unlike Study 1, this study was able to replicate the findings of Wang and Conway (2004) in that it demonstrated that interdependent cultures have more reflective comments in their everyday memories than do independent cultures. Wang and Conway espouse that those from interdependent cultures use more reflective comments because they "draw upon past events to convey moral messages, making reflective comments on how a memory event had taught them about life or the world" (p. 917). In trauma memories, however, independent culture had more reflective comments than did interdependent culture. Hence, the question is posed why do interdependent cultures not also reflect more in trauma memories? It is suggested that because the trauma memory is predominately autonomous there is little use reflecting on it in interdependent cultures as it does not reaffirm the relatedness self (and may in fact contradict the relatedness self). Those from interdependent cultures may also have less access to past autonomous events as reflective material. Furthermore, when recruiting participants for this research many interdependent participants made the comment "why would we remember a trauma event? We try to forget such events and 
move on'. Hence, there may be cultural differences in the appropriateness of trauma memory reflection.

PTSD status impacted on reflective comment. Those with PTSD provided more reflective comments than those without PTSD for both everyday and trauma memories. This is not surprising for the trauma memory given the impact/impairment the trauma has had on their life. PTSD may increase reflection on everyday memories as trauma has challenged beliefs and assumptions about life, world, self and others. Additionally, some argue (e.g., Resick \& Calhoun, 2001) that trauma prompts a search for meaning, especially in those with PTSD. It may also be the result of those with PTSD being more likely to attend counseling/therapy, where reflection is emphasized. Furthermore, this accords with current theoretical perspectives. Berntsen and Rubin $(2006,2007)$ posit that the trauma memory can become central to other autobiographical memories in those with PTSD. Ehlers and Clark (2000) suggest that in those with PTSD the trauma memory is biased by negative appraisals (such as permanent change) and in turn these people selectively retrieve information that is consistent with these appraisals. Thus, in both accounts reflection seems likely.

This study replicated previous findings (e.g., Wang \& Conway, 2004) demonstrating that interdependent culture have less specific everyday memories than independent cultures. Additionally, the study replicated Study 1 in that is was found that those from interdependent cultures had less specific trauma memories than did those from independent cultures. This finding suggests specificity is an important aspect of the structure of memory in reaffirming the conceptual self, which seems not to be moderated by memory theme. Furthermore, it was found that independent PTSD group provided less specific memories than did independent no PTSD, whereas those with and without PTSD from interdependent culture did not differ in terms of memory specificity overall. Specificity will be discussed further in the general discussion given its relevance to both Studies $3 \mathrm{a}$ and $3 \mathrm{~b}$.

In sum, Study 3a provided additional support for the conceptual framework in that the cultural distinction between autonomy versus relatedness evident for autobiographical remembering was not salient in autobiographical remembering of trauma. Rather the remembering of a traumatic event in trauma exposed individuals was predominantly guided by autonomous goals. The current study also demonstrated PTSD status moderates the impact of cultural differences in self on the level of autonomous orientation in the autobiographical remembering of everyday and trauma 
events. It was found that for independent culture trauma survivors with PTSD had less autonomous orientation in their autobiographical remembering than trauma survivors without PTSD. In contrast, it was found that for interdependent cultures trauma survivors with PTSD had more autonomous orientation in their autobiographical remembering than trauma survivors without PTSD. This accords with the additions to the conceptual framework that propose the more the trauma memory challenges the conceptual self the poorer the psychological adjustment. 


\subsection{Study 3b}

The hypothesis tested in Study 3b is that the interaction between culture and PTSD status will be significant in terms of autonomous orientation in the self-defining memories. Those from independent cultures with PTSD will have significantly less autonomous orientation in their self-defining memories than those from independent culture without PTSD. In contrast, those from interdependent cultures with PTSD will have significantly more autonomous orientation in their self-defining memories than those from interdependent culture without PTSD.

\subsubsection{Results}

\section{Content comparative analyses.}

Four culture (independent vs. interdependent) by PTSD status (PTSD vs. no PTSD) ANCOVAs, with depression and age as covariates, were conducted for each of the measures (theme, autonomous orientation, interaction scenario and other/self ratio). The means and standard deviations of these analyses are presented in Table 7.2.

\section{Table 7.2}

Means and (standard deviations) of variables scored in the self-defining memories of the four groups.

\begin{tabular}{lllll}
\hline & \multicolumn{2}{l}{ Independent culture } & \multicolumn{2}{l}{ Interdependent culture } \\
& PTSD & No PTSD & PTSD & No PTSD \\
\hline Theme & $.52(.25)$ & $.51(.27)$ & $.64(.26)$ & $.73(.29)$ \\
$\quad$ Relatedness & $.48(.25)$ & $.49(.27)$ & $.36(.26)$ & $.27(.29)$ \\
$\quad$ Autonomous & $8.08(4.92)$ & $12.26(7.02)$ & $5.34(3.48)$ & $3.13(2.67)$ \\
Autonomous orientation & $2.23(1.77)$ & $2.48(1.63)$ & $5.04(2.56)$ & $4.69(2.97)$ \\
Interaction scenario & $3.13(2.33)$ & $2.60(1.47)$ & $3.57(3.06)$ & $3.42(2.03)$ \\
Other/self ratio & & &
\end{tabular}

Theme. Independent culture provided more autonomous-themed and less relatedness-themed self-defining memories than did the interdependent group, $F$ (1, $100)=9.35, p<.01$. The PTSD group did not differ from the no PTSD group in terms 
of number of autonomous- and relatedness-themed self-defining memories, $F(1,100)$ $=1.04, p=.32$. The interaction was not significant, $F(1,100)=.67, p=.41$.

Autonomous orientation. Independent culture provided more autonomous orientation in their memories than did the interdependent group, $F(1,100)=40.06, p$ $<.01$. The PTSD group did not differ from the no PTSD group, $F(1,100)=1.61, p=$ .21. The interaction was significant, $F(1,100)=11.47, p<.01$. Independent PTSD provided less autonomous orientation than independent no PTSD, $t(55)=2.55, p=$ $.01, C I_{95}=-7.47,-.90, d=.69$. Whereas, interdependent PTSD provided more autonomous orientation than interdependent no PTSD, $t(47)=2.50, p=.02, C_{.95}=$ $.43, .3 .99, d=.71$.

Interaction scenario. Independent culture had less interaction scenarios in their self-defining memories than did the interdependent group, $F(1,100)=30.11, p$ $<.01$. The PTSD group did not differ from the no PTSD group, $F(1,100)=.02, p=$ 90. The interaction was not significant, $F(1,100)=.44, p=.51$.

Other/self ratio. Independent culture had significantly less other/self ratio than the interdependent group, $F(1,100)=3.95, p=.05$. The PTSD group did not differ from the no PTSD group, $F(1,100)=.34, p=.56$. The interaction was not significant, $F(1,100)=.17, p=.68$.

\section{Structure comparative analysis.}

Two separate $2 \times 2 \times 2$ way ANCOVAs with culture (independent vs. interdependent) and PTSD status (PTSD vs. no PTSD) as between subject factors, theme (autonomous vs. relatedness) as a within subject factor, and depression and age as covariates, with mean specificity and mean number of elaboration units as the dependent measures, were undertaken. Following this, a separate 2 × 2 x 2 way ANCOVA with culture (independent vs. interdependent) and PTSD status (PTSD vs. no PTSD) as between subject factors, experience (trauma vs. non-trauma) as a within subject factor, and depression and age as covariates, with mean number of elaboration units as the dependent measure was undertaken.

Specificity. Specificity of relatedness self-defining memories $(M=.44, S D=$ .42) was significantly greater than specificity of autonomous self-defining memories $(M=.41, S D=.44), F(1,100)=4.29, p=.04$. Independent culture $(M=.55, S D=$ .42) had significantly more specific self-defining memories than did interdependent group $(M=.28, S D=.39), F(1,100)=16.90, p<.01$. The interaction between 
memory theme and culture was not significant, $F(1,100)=.00, p=.95$. The PTSD group did not differ from the no PTSD group in terms of specificity, $F(1,100)=.80$, $p=.37$. The interaction between culture and PTSD status was significant, $F(1,100)=$ $7.30, p<.01$. Independent culture without PTSD $(M=.66, S D=.40)$ provided significantly more specific memories than independent culture with PTSD $(M=.43$, $S D=.43), t(55)=2.09, p=.04, C I_{.95}=.01, .45, d=.55$. However, interdependent culture with $(M=.34, S D=.39)$ and without PTSD $(M=.21, S D=.38)$ did not differ, $t(47)=1.18, p=.24, C I_{.95}=-.35, .09, d=.34$. The interaction between memory theme and PTSD status was not significant, $F(1,100)=.25, p=.62$, or the three-way interaction, $F(1,100)=.00, p=.97$.

Elaboration. Elaboration of relatedness and autonomous memories did not differ, $F(1,100)=.94, p=.34$. Independent culture did not differ from interdependent culture in terms of elaboration, $F(1,100)=.15, p=.70$. However, the interaction between memory theme and culture was significant, $F(1,100)=6.06, p=$ .02 . Interdependent culture $(M=9.48, S D=5.40)$ tended to provide significantly more elaborate relatedness memories than did independent culture $(M=7.78, S D=$ 4.91), $t(104)=1.70, p=.09, C I_{95}=-.29,3.69, d=.33$. However, independent $(M=$ $7.91, S D=5.15)$ and interdependent culture $(M=6.50, S D=6.50)$ did not differ in terms of elaboration of autonomous memories, $t(104)=1.25, p=.22, C I_{95}=-3.66$, $.84, d=.24$. The independent group did not differ in elaboration of their autonomous and relatedness memories, $t(56)=.20, p=.42, C I_{.95}=-1.33,1.09$, but the interdependent group provided significantly more elaborate relatedness memories than autonomous memories, $t(48)=3.31, p<.01, C I_{.95}=1.17,4.78$. The PTSD group $(M=8.73, S D=5.99)$ did not differ from the no PTSD group $(M=7.19, S D=4.91)$ in terms of elaboration, $F(1,100)=1.84, p>.05$. The interactions between culture and PTSD status, $F(1,100)=.03, p=.87$, memory theme and PTSD status, $F(1$, $100)=.05, p=.83$, and the three-way interaction, $F(1,100)=.02, p=.90$, were not significant.

In terms of trauma and non-trauma memory elaboration, non-trauma memories tended to be more elaborate than trauma memories, $F(1,100)=3.82, p=.05$. Independent culture did not differ from interdependent group in the elaboration of these memories, $F(1,100)=1.96, p=.17$. However, the interaction between memory theme and culture was significant, $F(1,100)=9.48, p<.01$. Interdependent $(M=$ 9.71, $S D=6.87)$ and independent culture $(M=8.12, S D=5.02)$ did not differ in 
elaboration of non-trauma memories, $t(104)=1.37, p=.17, C I_{.95}=-.71,3.89, d=$ .26. Independent culture $(M=6.41, S D=6.53)$ did provide more elaborate traumathemed memories than did interdependent culture $(M=2.49, S D=4.91), t(104)=$ $3.21, p<.01, C I_{95}=1.39,5.91, d=.63$. The PTSD group $(M=7.24, S D=6.96) \mathrm{did}$ not differ from the no PTSD group $(M=6.13, S D=5.02)$ in terms of elaboration, $F$ $(1,100)=1.07, p=.30$. The interactions between culture and PTSD status, $F(1,100)$ $=1.24, p=.27$, memory theme and PTSD status, $F(1,100)=1.64, p=.20$, and the three-way interaction, $F(1,100)=.86, p=.36$, were not significant.

\subsubsection{Discussion}

Study $3 \mathrm{~b}$ provided additional support for the proposed mechanisms accounting for the relationship between the nature of the trauma memory and PTSD. The study demonstrated that culture and PTSD status interact in terms of the level of autonomous orientation in self-definition. Specifically, those from independent culture with PTSD provided less autonomous orientation in their self-defining memories than did those without PTSD. In contrast, those from interdependent culture with PTSD provided more autonomous orientation in their self-defining memories than did those without PTSD. This finding extends the finding of Study $3 \mathrm{a}$ in that it suggests that not only does PTSD and culture interact in terms of the level of autonomous orientation in autobiographical remembering but also in key, self-focused memories that provide definition for the self. Additionally, as self-defining memories may provide a constructive approach to studying change in self-concept (Sutherland \& Bryant, 2006), such findings support the notion that the level of autonomous orientation in the trauma memory may impact on the development, expression and maintenance of the conceptual self.

Furthermore, independent culture provided more autonomous-themed, more autonomously oriented self-defining memories with more interaction scenarios and references to the self than interdependent culture. This is evident in the following examples.

1. Becoming a Christian when I was 6. I decided to give my life to God.

2. Being baptised and confirmed. That was a public declaration that showed I am living for God. 
3. Being nominated for Art Express. This was an exciting time where I was encouraged and even given credit for creative gifts.

4. When I was made prefect at school. This gave me the chance to be a leader at school. It showed me that people recognised my gifts.

5. Getting into university. This made me very excited and happy. It was a fresh start and is something I want to be doing.

\section{- Independent no PTSD}

1. I am my parents' eighth child and fourth daughter. My family was very orthodox. They believe in fate.

2. Government policy changes everyday (in China) people worry about new policies.

3. My husband is our best choice. He is a good man. He always respects my feelings. He gave everything.

4. We are having our $10^{\text {th }}$ wedding anniversary. We are a good couple. We respect each other.

5. We are a very lucky couple. We got one boy and one girl. We have everything we wished for.

\section{- Interdependent no PTSD}

Replicating Study 1, the findings suggest that cultural differences in the elaboration of autobiographical memories depend on the theme of the memory and non-trauma memories were found to be more elaborate than trauma memories, further confirming theoretical approaches (e.g., Conway, 2005; Ehlers \& Clark, 2000) that posit poor elaboration of the trauma memory. Unlike Study 1, the hypothesis suggesting independent culture would elaborate trauma memories more than interdependent culture, given trauma memories' emphasis on autonomy and independent cultural acceptance of the trauma memory, was supported. The impact of requesting participants to elaborate trauma memories on the psychological and cultural adjustment of trauma survivors from interdependent cultures, however, remains unknown.

Similar to all previous studies, independent culture provided more specific memories than did interdependent culture suggesting specificity to be a memory retrieval technique essential in the re-affirmation of the self as an autonomous 
entity (Wang \& Conway, 2004) and hence, utilized by independent societies in retrieval of all autobiographical memories. Furthermore, replicating Study 3a it was found that independent PTSD group provided less specific memories than did independent no PTSD, whereas those with and without PTSD from interdependent culture did not differ in terms of memory specificity.

In sum, Study $3 b$ demonstrated that the relationship between PTSD and the level of autonomous orientation in self-definition is culturally specified. Such findings support proposals that the level of autonomous orientation in the trauma memory impacts on the development, expression and maintenance of the conceptual self. Furthermore, independent culture provided more autonomous-themed and more autonomously oriented self-defining memories with more social interactions and references to the self than interdependent culture. Replicating Study 1, the findings suggest that cultural differences in the elaboration of autobiographical memories depend on the theme of the memory. 


\subsection{Study $3 c$}

The hypothesis tested in Study $3 \mathrm{c}$ was that those from independent cultures with PTSD will have significantly more trauma-themed goals, self-defining memories and self-cognitions than those from independent cultures without PTSD. However, those from interdependent cultures with PTSD will not describe a trauma-themed selfconcept and hence, will not differ from those from interdependent cultures without PTSD, regarding trauma-centered goals, self-definition and self-cognition.

\subsubsection{Results}

\section{Trauma comparative analyses.}

The means of the self-concept measures are presented in Figure 7.4. Given the hypothesis, only the interactions have been reported. Significant interactions were followed up with two planned independent t tests; independent PTSD versus independent no PTSD and interdependent PTSD versus interdependent no PTSD.

Three independent culture (independent vs. interdependent) by PTSD status (PTSD vs. no PTSD) ANCOVAs of self-concept (i.e., goals, self-defining memories and self-cognitions) that controlled for depression and age indicated significant culture by PTSD status interaction effects for goals, $F(1,100)=11.33, p<.01$, selfdefining memories, $F(1,100)=4.04, p<.05$, and self-cognitions, $F(1,100)=15.84$, $p<.01$.

Planned follow-up comparisons using the adjusted means indicated that independent culture with PTSD, when compared to independent culture without PTSD, had significantly more trauma-themed personal goals, $t(55)=2.93, p=.01$, $C I_{.95}=.11, .34, d=.99$, self-defining memories, $t(55)=3.16, p<.01, C I_{.95}=.12, .40$, $d=.98$, and self-cognitions, $t(55)=3.85, p<.01, C I_{.95}=.05, .14, d=1.14$. However, interdependent culture with PTSD and without PTSD did not differ in terms of trauma-themed goals, $t(47)=1.39, p=.17, C I_{.95}=-.00, .01, d=.00$, self-defining memories, $t(47)=.61, p=.54, C I_{.95}=-.10, .20, d=.82$, and self-cognitions, $t(47)=$ $.57, p=.57, C I_{.95}=-.01, .01, d=.50$. 


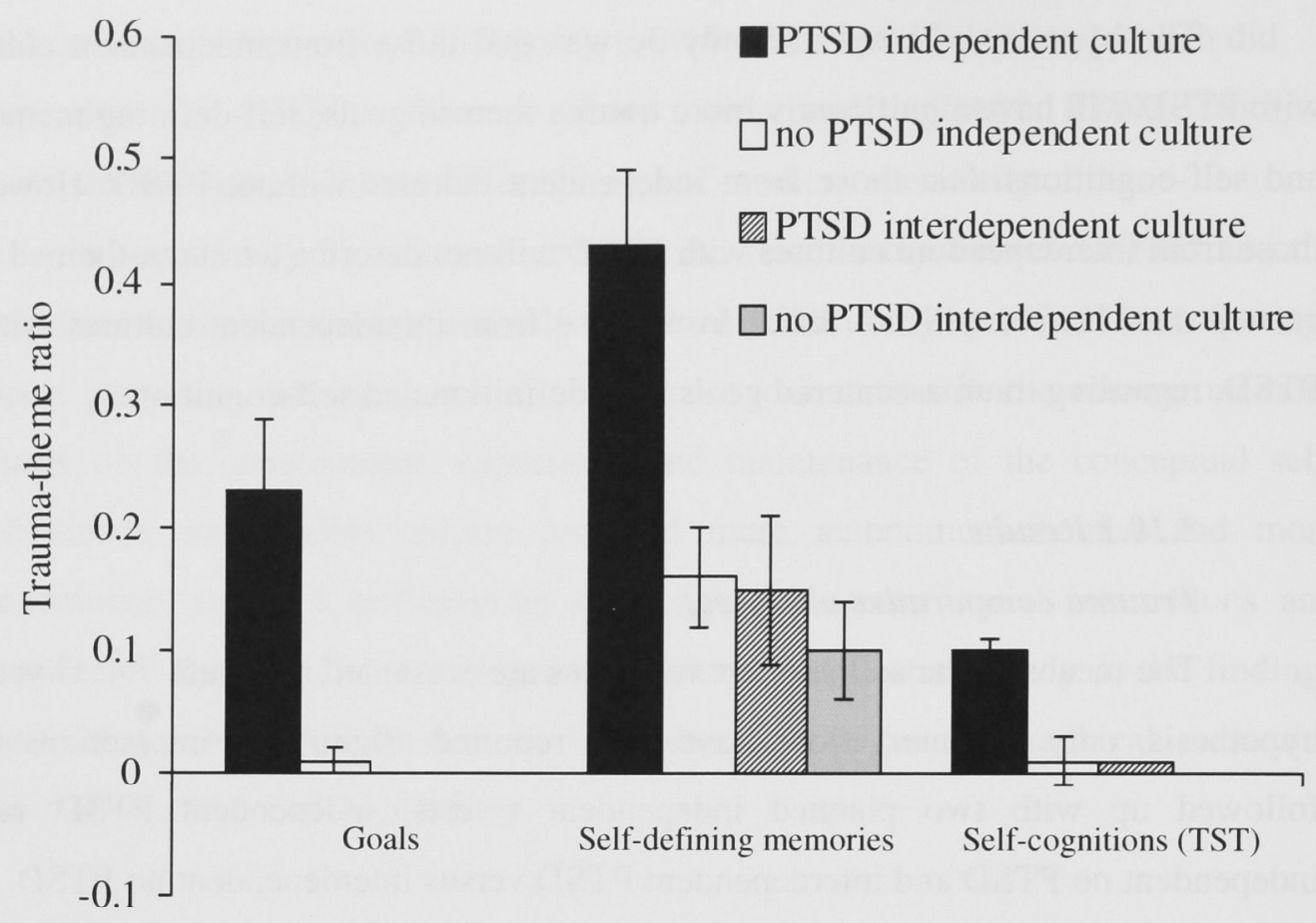

Note. The PTSD interdependent and no PTSD interdependent groups had trauma-theme ratio means of $.00(S D=.01, .00$, respectively) for the goals measure and the no PTSD interdependent group had a trauma ratio mean of $.00(S D=.02)$ for the self-cognitions measure. Error bars not shown were too small to depict.

Figure 7.4 Adjusted means for the trauma ratios for goals, self-defining memories and self-cognitions. Error bars indicate \pm 1 SE of the mean.

\subsubsection{Discussion}

This study extended Study 1c in demonstrating that trauma's impact on change in self-definition and personal identity in criteria A trauma exposed individuals is culturally specified. This has now been demonstrated in a sample that is a trauma exposed community sample with more ecologically validity and greater clinical relevance than a university sample. While the findings are consistent with those of other studies that show trauma memory can become salient in self-conception and thus an important component of personal identity and self-definition in PTSD, they also show that this alteration in self-concept and identity is not the case for those from interdependent cultures. Trauma exposed participants from independent cultures with PTSD clearly displayed a more trauma-defined personal identity than those 
without PTSD. For those from interdependent cultures, however, there were no differences in trauma-centered identity between the PTSD and no PTSD groups. This was found using three different personal identity measures - a goals measure, a selfdefining memory measure and a self-cognition measure. 


\subsection{General Discussion}

Study 3 examined if PTSD moderates cultural differences in autobiographical memory and trauma's impact on personal identity in a group of participants that were a clinically relevant group. The objective of this discussion is to briefly summarize the main findings and focus primarily on the theoretical and clinical implications of Study 3.

\subsubsection{Summary of Findings}

In general, the findings supported the first hypothesis that the cultural distinction between autonomous versus relatedness orientation would be evident in everyday memories but not in trauma memories. The prediction that autobiographical memories of trauma for both independent and interdependent cultures would be predominately autonomous was supported. Trauma survivors from independent cultures had greater autonomous orientation in their everyday memories than trauma survivors from interdependent cultures. However, trauma survivors from interdependent cultures had equally as autonomously oriented trauma memories as trauma survivors from independent cultures.

Second, the hypothesis suggesting the level of autonomous orientation would culturally differ in terms of differentiating between those with and without PTSD was supported. It was found that trauma survivors from independent cultures with PTSD had lower levels of autonomous orientation in their autobiographical memories of trauma and everyday events than trauma survivors from independent cultures without PTSD. In contrast, trauma survivors from interdependent cultures with PTSD had higher levels of autonomous orientation in their autobiographical memories of trauma and everyday events than did trauma survivors from interdependent cultures without PTSD.

The third hypothesis was also supported. Trauma survivors from independent cultures with PTSD had significantly less autonomous orientation in their selfdefining memories than trauma survivors from independent cultures without PTSD. In contrast, trauma survivors from interdependent cultures with PTSD had significantly more autonomous orientation in their self-defining memories than trauma survivors from interdependent cultures without PTSD 
The final hypothesis was supported. Trauma exposed individuals from independent cultures with PTSD had significantly more trauma-themed goals, selfdefining memories and self-cognitions than trauma exposed individuals from independent cultures without PTSD. However, trauma exposed individuals from interdependent cultures with PTSD did not describe a trauma-themed self-concept.

\subsubsection{Theoretical Implications}

\section{Cultural differences in autobiographical remembering and PTSD.}

The findings support predictions regarding the relationship between cultural differences in autobiographical remembering and PTSD outlined in the introduction. First, throughout this thesis it has been theorized that trauma events by their nature, primarily activate an autonomous hierarchy of goals, regardless of the culturally dominant self-system. Such a theoretical position was supported in this study where the autobiographical remembering of everyday events reflected cultural variation in self-construal, while trauma survivors provided autonomously-oriented trauma memories, regardless of their dominant self-construal.

Second, based on current theoretical stances, Study 3 proposed that people have schema-driven expectations as to appropriate levels of autonomous orientation in autobiographical remembering which is derived from the conceptual self. Specifically, for those from independent culture, emphasis on an independent conceptual self results in schema-driven expectations of high levels of autonomous orientation in autobiographical remembering. In contrast, for those from interdependent culture emphasis on interdependent conceptual self results in schemadriven expectations of low levels of autonomous orientation in autobiographical remembering. Hence, it was suggested that the levels of autonomous orientation in the trauma memory has the potential to violate schema-driven expectations as to appropriate levels of autonomy in autobiographical remembering. Based on theoretical considerations (Berntsen \& Rubin, 2007) and empirical findings (e.g., Brewer \& Treyens, 1981) it was predicted that such violations of schema-driven expectations would result in enhanced memory of the trauma event and thus, as Berntsen and Rubin (2007) assert the trauma autobiographical memory forms "reference points for the organization of other less significant experiences" (p. 418). Berntsen and Rubin propose that if trauma memories do become central to the organization of autobiographical memory, it is likely to result in PTSD. In support of 
this proposal, it was found that trauma survivors from independent cultures with PTSD had significantly less autonomous orientation in their trauma memories than those from independent cultures without PTSD, while trauma survivors from interdependent cultures with PTSD had significantly more autonomous orientation in their trauma memories than those from interdependent cultures without PTSD. Admittedly, it is possible that those from independent cultures with lower levels of autonomous orientation in their autobiographical remembering are more prone to PTSD, while those from interdependent cultures with higher levels of autonomous orientation in their autobiographical remembering are more prone to PTSD. However, there seems to be no theoretical or empirical support for this hypothesis.

One potential outcome of centrality of the trauma memory is an impact of the trauma memory on the functional role of autobiographical memory. Wang and Conway (2004) assert that autobiographical memories are instrumental in the development, expression and maintenance of the conceptual self. Therefore, if the trauma memory becomes the cognitive reference point for other autobiographical memories, it seems likely that the level of autonomous orientation in the trauma memory will impact on the expression, development and maintenance of the conceptual self. Consequently, the conceptual self is threatened and altered. Support for this proposal comes from the finding that trauma survivors from independent cultures with PTSD had significantly less autonomous orientation in their selfdefining memories (a measure of alteration in the conceptual self) than those from independent cultures without PTSD, while trauma survivors from interdependent cultures with PTSD had significantly more autonomous orientation in their selfdefining memories than those from independent cultures without PTSD.

Additionally, Wang and Conway (2004) theorize that the conceptual self is used to encode, organize and retrieve autobiographical memories. Therefore, it seems likely that this threat to the conceptual self will impact on on-going encoding, organization and retrieval of other autobiographical memories, such as memories of everyday events. This impact of the conceptual self on on-going autobiographical remembering is thought to maintain threat to the conceptual self. This proposal was supported by the finding that trauma survivors from independent cultures with PTSD had significantly less autonomous orientation in their everyday memories than those from independent cultures without PTSD, while trauma survivors from interdependent 
cultures with PTSD had more autonomous orientation in their everyday memories than those from interdependent cultures without PTSD.

\section{Threat to the conceptual self.}

Like, Ehlers and Clark (2000), it is thought that those with PTSD tend to appraise current situations as an on-going threat. This threat commonly is an internal threat to the conceptual self. Ehlers and Clark posit that once the perception of current threat is activated it is accompanied by PTSD symptoms and the perceived threat prompts behavioral and cognitive responses that aim to "reduce perceived threat and distress in the short-term, but have the consequence of preventing cognitive change and therefore maintaining the disorder" (p. 320). It is suggested that threat to conceptual self can be direct (i.e., lower levels of the expected self) or indirect (i.e., higher levels of the culturally inappropriate self).

Therefore, for trauma survivors with an independent conceptual self, low levels of autonomous orientation in autobiographical remembering has the potential to pose a direct threat to the conceptual self as personal control over the environment, a sense of achieving personal success and asserting the self has diminished. Additionally, it is suggested that independent cultures will be less tolerant of a trauma survivor with a lessened sense of autonomy (Sato, 2001). This may impair the survivor's ability to function properly as an autonomous, independent member of society and may also result in social alienation. In contrast, for trauma survivors with an interdependent conceptual self high levels of autonomous orientation in autobiographical remembering has the potential to indirectly threaten the conceptual self as an increased emphasis on autonomy (i.e., increased self-importance and personal control) undermines the self as interdependent, fitting in, maintaining group harmony and fulfilling social obligations. Additionally, it is proposed that interdependent cultures will be less tolerant of a trauma survivor with a lessened sense of relatedness. Such a social response has the potential to result in alienation, a rejection by significant others and a lack of social support. Such outcomes have been associated with poor psychological adjustment.

\section{Identity and self-definition.}

The thesis predicted that another potential outcome of the trauma memory becoming the cognitive reference point for other autobiographical memories is that trauma becomes central to identity, self-concept and self-definition. Theorists (e.g., Conway, 2005) propose that autobiographical memories are a constituent of the self 
and identity. Therefore, it was proposed that centrality of the trauma autobiographical memory is most likely to be regarded as a central component of personal identity and the self. Moreover, the centrality of trauma approach (Berntsen \& Rubin, 2007) and SMS (Conway, 2005) posit that trauma-centered self-change is a potential outcome following trauma and that the need for self-consistency and self-coherence is instrumental in the move towards trauma-centered self-definition. It was hypothesized that such self-change is contingent on a requirement for self-consistency and selfcoherence. Research (Suh, 2000, 2002) has demonstrated that self-consistency needs are culturally variable. Therefore, it was predicted that the determining factor as to whether the trauma becomes central to identity is the requirement for self-consistency. That is, if the trauma survivor has a requirement for self-consistency and is defined by a set of stable internal attributes, it is likely that a trauma memory central to the life story and other autobiographical memories, results in identity or conceptual self change. Again identity or conceptual self change will threaten the conceptual self, as the conceptual self becomes centered round trauma. The findings supported such proposals in that a trauma-centered identity differentiated between those with and without PTSD from independent cultures but trauma survivors with and without PTSD from interdependent cultures did not differ in terms of trauma-centered identity.

The finding also supports the centrality of trauma approach (Berntsen \& Rubin, 2007) and SMS (Conway, 2005) in that the need for self-consistency and selfcoherence is instrumental in the move towards trauma-centered self-definition. However, the findings suggest such theories consider and make explicit cultural variability in self-consistency needs. Additionally, the results accord with the proposal outlined in Study 1 that the social role of trauma victim/survivor is a culturally sanctioned role in independent cultures given its alliance with cultural expectations of the self, personal identity and the individual life story. However, in interdependent cultures the social role of trauma survivor/victim may be viewed as culturally inappropriate, as it contradicts cultural expectations of a communal self. The findings point to important cultural considerations both in the etiology of trauma-centered personal identity in PTSD and in its maintenance, which are not part of current psychological models of PTSD.

Furthermore, Berntsen and Rubin (2007, p. 221) state that "salient personal memories form turning points" and "if a trauma memory is seen as a central turning 
point in our life story it would also most likely be regarded as a central component of our personal identity". However, combining the results of Studies 3a, b and c, suggests this assertion may be culturally dependent as it seems that the trauma memory can become a cognitive reference point for the organization of other autobiographical memories (Study $3 \mathrm{a}$ and b) without becoming the salient and important component of identity (Study 3c). Hence, alterations in identity are not necessarily the consequence of the trauma memory becoming a salient autobiographical memory. It is recommended that their approach clarify this distinction; trauma memory centrality may not always result in identity change, especially in interdependent cultures.

\subsubsection{Clinical and Forensic Implications}

\section{Autonomous orientation.}

The finding of PTSD status and culture interacting in terms of autonomous orientation has clinical implications. Herman (1992) proposes that treatment needs focus on empowering the survivor and assisting the survivor in establishing new relationships. She claims that the "first principle of recovery is the empowerment of the survivor" and that the efforts of "others may offer advice, support, assistance, affection, and care, but not cure" (p. 133). The role of others is to help the survivor in “rebuilding a positive view of the self" (Herman, 1992 p. 63). Ehlers et al. (2000) also suggest the role of others is to assist the survivor in correcting negative beliefs about themselves and others. Herman believes that it is not until this regaining of autonomy has been achieved that a focus on "deepening relationships" with peers, lovers and family should be the focus of treatment (p. 205). This approach aligns with the findings, as the results suggest that in independent cultures therapy may need to focus on increasing autonomous orientation in clients with PTSD. Practically, this may involve exposure work that highlights and focuses on autonomous aspects of the memory. It may also involve clients repeatedly re-telling the trauma event (either verbally or in writing) with increased autonomous orientation (i.e., the client includes more personal like, dislikes, evaluations, agency, uniqueness, etc. in the personal retelling of the event).

However, the results clearly highlight the autonomous orientation of these therapeutic approaches. The results suggest that primary focus on agency and autonomous orientation in interdependent cultures may be less relevant. Instead the 
focus of therapy with clients from interdependent cultures may need to center round re-framing and/or reducing the level of autonomous orientation and increasing levels of relatedness. In interdependent cultures this may translate to a downplaying of autonomous aspects of the memory during exposure work and clients re-telling the trauma event (either verbally or in writing) with decreased autonomous orientation (i.e., the client includes less personal like, dislikes, evaluations, agency, uniqueness, etc. in the personal re-telling of the event) and/or increased emphasis on relatedness aspects of the memory (i.e., interdependence with others, a focus on the feelings, thoughts and behaviors of others during the trauma, and the clients social roles during the trauma). Cognitive work may also involve a cognitive re-structuring of the memory to realign the memory with the conceptual self.

Therefore, agreeing with Herman (1992), this thesis does propose that treatment needs focus on empowering the survivor (autonomy) and assisting the survivor in establishing new relationships (relatedness), given these two aspects of self are fundamental for well-being, mental health and operating in effective ways with the world (Sato, 2001). However, the findings suggest Herman's advice may be culturally dependent. This may be true enough of individualistic cultures but it is argued that in collectivistic cultures (and even in individuals from individualistic cultures holding strong interdependent self-construal) the first principle of recovery may not be the empowerment of the survivor but rather to focus on the roles and efforts of others in offering advice, support, assistance, affection, and care towards "deepening relationships" with significant others. Further research (including clinical treatment studies) is required to examine the efficaciousness of such proposals.

\section{Identity and self-definition.}

The finding of cultural moderation of trauma's impact on personal identity has clinical implications. The finding suggests that while a clinical awareness of the impact of trauma on identity and self-definition is important, awareness needs also consider cultural factors which moderate any impact. Currently, the clinical awareness remains strongly individualistically focused (Bracken, 2002). The impact of trauma on identity and self-definition is explored at assessment and addressed in self-schema work. This work aims to address trauma caused "vulnerable identities" such as "the self as powerless...inferior...nonexistent...futureless" (Brewin, 2003, p. 73), to integrate current views of the self (e.g., I am a victim, I am damaged, I have no control) into existing self-knowledge and the life story, and to make sense of the 
trauma in respect to existing aspects of their self-concept and goals (Hembree \& Foa, 2004), to achieve self-consistency. The results of the current studies strongly suggest that the list of vulnerable "identities", which is mostly comprised of private components of the self, needs to be extended to include public and collective components of the self, such as social roles, the impact of trauma on such social roles and the value of role complexity and diversity. Additionally, it needs to be considered that identity may not always be "vulnerable", especially in interdependent cultures.

These considerations align with clinical observations. While some trauma survivors from interdependent cultures stated that they were no longer the same following trauma, attempts to explore and compare pre- and post-trauma self-views in an effort to highlight possible similarities that would demonstrate self-consistency, failed. These clients' focus and distress seemed unrelated to changes in private aspects of self but rather due to social role changes such as "I am a dependent refugee", "I am no longer protector of my family", "I am no longer working", "I am a receiver of government benefits". Furthermore, it has been observed that for some clients from interdependent cultures, their major concern in therapy is centered round a dissatisfaction in current social role distribution such as "being too much a son and not enough a husband, friend and father" and wanting to regain balance in such social roles. Additionally, from further a field, a sign on a wall outside a classroom in Aceh Indonesia in response to the tsunami in 2004 , where more than $75 \%$ of the people living in that area had been killed, missing or made homeless, stated "'I am still as I am before, I am still studying, studying hard for my future" (Grady \& Lipton, 2005).

In addition, it must be considered in assessment and therapy that in interdependent cultures discussing issues pertaining to the private self may be not only unhelpful but also viewed as immature and inappropriate. There also must be consideration of an emphasis on the communal self resulting in less acceptance, tolerance, discussion and in turn assistance of trauma survivors of particular trauma types that disrupt the group and community, such as rape and domestic violence. Members of interdependent cultures may have reason to suppress trauma-related cognitions pertaining to such trauma types due to these cultural sanctions.

However, the findings do not dismiss the usefulness of cognitive behavioral therapy in interdependent cultures. Effective components such as imaginal exposure may not be as influenced by cultural factors, techniques such as goal-setting in life roles using behavioral activation models such as those used for depression (e.g., 
Lejuez, Hopko, \& Hopko, 2001) seem appropriate and cognitive therapy remains useful in addressing aspects of the trauma experience and appraisals that are not culturally sanctioned. Furthermore, there is growing support (e.g., Schwartz, 2005; Tarrier \& Humphreys, 2003) for the use of interventions that target social support as adjuncts to cognitive behavior therapy in the treatment of PTSD. Practically this suggests an inclusion of a client's interpersonal and social context in case formulation (Tarrier, 2006), an acknowledgment of the impact of the beliefs held by significant others on the development and maintenance of PTSD (Tarrier \& Humphreys, 2003), and the consideration of treatment options that are designed to facilitate and improve social support (Harvey, Bryant, \& Tarrier, 2003) such as family therapy, reengagement with social networks, modification of perceptions about the intentions and reactions of others, and a focus on reciprocity and social exchange (Tarrier \& Humphrey, 2003). Therapy sessions may involve the inclusion of family members, significant others (Glynn et al., 1999) and community members.

\section{Specificity.}

Consistently in Study 3, it was found that trauma survivors from interdependent cultures have less specific memories (everyday, trauma and selfdefining) than trauma survivors from independent cultures. This finding alongside the findings of Study 1 suggests specificity is an important technique in reaffirming the conceptual self, which seems not to be moderated by memory theme.

A forensic implication of this specificity finding is derived from Herlihy and Turner's (2002) remark that the more detail a memory has the more believable and credible the memory. General memories are thus viewed as not credible, false or exaggerated and thus are thought to have not been recalled clearly. The common thought is that general details can be easily constructed (from historical and schematic information) whereas specific details cannot. Therefore, the level of specificity has been seen as a good way of distinguishing between accurate and inaccurate memories (Herlihy \& Turner, 2002). Memory recollection is essential in court/legal settings and especially refugee tribunal hearings. Experimental (e.g., Christianson \& Safer, 1996), clinical (e.g., McNally et al., 1995; Williams \& Dritchel, 1988) and refugee studies (e.g., Herlihy \& Turner, 2002) challenge this in that their findings suggest emotion impacts on memory specificity. This study indicates that culture also impacts on specificity and hence, needs to be considered when deeming an autobiographical memory as credible or not. 
The finding also has clinical implications. Greater time and resources may be required to gather specific details during the assessment and therapy (cognitive and exposure) stages when working with clients from interdependent culture. However, research is needed to explore if specific details of the trauma memory are required in therapies (such as exposure work) with interdependent clients and the impact of providing specific memories on psychological and cultural adjustment.

It was also found that the independent group with PTSD provided significantly less specific everyday memories than the independent group without PTSD, even when depression was controlled for. This accords with the accumulating research (e.g., Hauer, Wessel, \& Merckelbach, 2005; McNally et al., 1995; Raes, Hermans, Williams, \& Eelen, 2005; Raes et al. 2006) suggesting that those with PTSD tend to report overgeneral autobiographical memories. Hermans et al. (2004) suggest that recalling overgeneral autobiographical memories is functional in the short-term for those with PTSD as it assists in the regulation of affect and prevents the recall of painful memories but is not as effective in the long-term as it can maintain emotional disorders. This approach thus predicts that given those from collectivistic cultures tend to always provide general memories (regardless of the theme of the memory) there would be no differences in specificity between those with and without PTSD. Supporting this, the study found that those with and without PTSD from interdependent culture did not differ in level of specificity of their trauma or everyday memories. However, this finding does question the notion that high memory specificity is related to emotional health (Hermans et al., 2004), as the results indicate that people (i.e., those from interdependent cultures) can maintain high levels of emotion health whilst retrieving general memories. Further research should explore such a relationship.

\subsubsection{Limitations}

The shortcomings of this study are acknowledged. First, a major limitation of this study was in the method of PTSD diagnosis. The study would be improved if the self-report questionnaire (PDS) used for diagnostic purposes was followed up with a structured interview. Second, self-completion at home may have biased results such as participants discussing responses with others producing possible culturally desirable responses. Third, as in any study exploring the impact of culture on certain variables, language and task understanding must be considered. The finding of no 
cultural differences in the self-report of task difficulty was taken to suggest that there were no major cultural differences in task understanding and responding. However, retrieving memories and self-cognitions in English may impact on how identity is presented. Furthermore, this study was conducted in Australia, an independent cultural environment. This may result in an intracultual context for the interdependent groups but an intercultural context for the interdependent groups. This was minimized by allowing all participants to complete the study at home and including migrants in both groups. Fifth, there is an acknowledgement of possible demographic differences (e.g., education, economic sufficiency etc.) between groups which may have confounded the cultural independent variable. While there is little evidence that such factors would affect autobiographical remembering and identity it may be helpful for groups to be more closely matched in further research. Further, it is uncertain whether level of autonomous orientation is a cause or consequence of PTSD. Hence, prospective research is required. Additionally, it cannot be certain to what extent accurate variations in self-concept was measured as opposed to what people were willing to disclose about self-concept. Finally, it is acknowledged that the independent/interdependent construct is only one cultural dimension and the cultures comprising these groups in this study vary on other cultural dimensions.

\subsubsection{Conclusion}

Despite these limitations, it is believed that this study is an important and timely one that demonstrated a key cultural difference in the impact of the nature of autobiographical remembering on PTSD status and in the impact of trauma on personal identity. Those from independent cultures with PTSD had significantly lower levels of autonomous orientation in the autobiographical remembering of trauma, everyday and self-defining memories and greater trauma-centered personal identity when compared to those from independent cultures without PTSD. In contrast, those from interdependent cultures with PTSD had significantly higher levels of autonomous orientation in the autobiographical remembering of trauma, everyday and self-defining memories than those without PTSD and there was no relationship between trauma-centered personal identity and psychological adjustment. These findings suggest our current PTSD models and their theoretical assumptions need to consider cultural factors in the nature of the self in any theoretical reformulation. If these findings are robust, the clinical implications are significant. In particular, they 
would question the relevance of the focus on self-referential thinking, which is an integral part of assessment and treatment in some cognitive therapies for PTSD. For example, it would suggest a lesser role for cognitive reframing of self-schema and autonomous orientation for those from interdependent cultures and a greater emphasis on the impact of trauma on the public/collective aspects of self for those from interdependent cultures. Given interdependent cultures do suffer from PTSD, future research is required, for both theoretical and clinical reasons, to explore processes other than trauma's impact on self-definition that may be maintaining PTSD in such populations. 


\section{CHAPTER 8}

\section{Study 4: Cultural Differences in Cognitive Appraisal Styles Utilized During Trauma in those with and without PTSD}

Study 2's objectives were to explore the impact of cultural differences in self on the cognitive appraisal styles of trauma and on the relationship between the cognitive appraisal styles and psychological adjustment. Additionally, it aimed to investigate the cultural appropriateness of Ehlers and Clark's (2000) appraisal model. Due to inconsistent results and methodological concerns, Study 2 only provided a very preliminary analysis of these objectives. The objective of Study 4, therefore, is to re-analyze the trauma narratives generated in Study 3 examining the impact of cultural variation in self on the relationship between cognitive appraisal styles and PTSD. Study 4 also addresses the methodological limitations of Study 2 by using a large sample comprised of trauma survivors with and without PTSD from independent and interdependent cultures; having participants complete the data booklet at home reducing intercultural context effects; and by controlling for trauma type, severity and duration.

\subsection{Hypotheses}

It is predicted that:

1/ there will be a lack of cultural differences in the cognitive appraisal styles (mental defeat/ lack of psychological autonomy, control strategies) utilized during trauma, while there will be evidence for cultural differences in the cognitive appraisal styles (alienation, permanent change) generated post-trauma. Therefore, it is predicted that while the culture main effects for mental defeat and control strategies will not be significant, the culture main effects for alienation and permanent change will be significant,

$2 /$ the impact of the cognitive styles on psychological adjustment will differ culturally, depending on the threat the appraisal poses to the conceptual self. The interactions between culture and PTSD status will be significant for mental defeat, control strategies, alienation and permanent change. Specifically, for independent culture, those with PTSD will have greater mental defeat appraisals, fewer control strategies and greater permanent change appraisals than those without PTSD from independent 
culture. Whereas, for the interdependent group, this difference will not be as significant, and

$3 /$ for the interdependent group those with PTSD will have greater alienation appraisals than those without PTSD from interdependent culture. However, this difference will not be as significant for the independent group.

\subsection{Method}

\subsubsection{Participants}

Participants were recruited as outlined in Study 3.

\subsubsection{Procedure}

The procedure is outlined in Study 3. This study re-analyzed the trauma narratives collected in Study 3a.

\subsubsection{Scoring/Coding System}

Narratives were scored using the criteria outlined in Study 2. That is, the trauma narratives coded mental defeat, control strategies, alienation and permanent change as was done in Study 2.

\subsubsection{Reliability}

A second independent rater who was Chinese coded $20 \%$ of trauma narratives. Raters were blind to the cultural group and PTSD status of participants. The second rater was blind to the study hypotheses. Kappa coefficients were adequate for mental defeat $(\kappa=.75)$, control strategies $(\kappa=.82)$, alienation $(\kappa=.71)$ and permanent change $(\kappa=.73)$. Discrepancies between raters were resolved through discussion.

\subsection{Results}

\subsubsection{Participant and Group Characteristics}

Participant and group characteristics are outlined in Study 3. 


\subsubsection{Preliminary Analysis}

As outlined in Study 3, the cultural groups and the PTSD status groups were equivalent in terms of Criteria A trauma distribution as indicated on the PDS, trauma history (THQ), self-reports of trauma severity and self-reported time of the trauma event.

\subsubsection{Comparative Analysis}

To examine cultural differences in the four cognitive appraisal styles four culture (interdependent vs. independent) $\mathrm{x}$ PTSD status (PTSD vs. no PTSD) ANCOVAs were conducted with age and depression as covariates and each cognitive appraisal style as the dependent variable. As done in Study 2, culture main effects were one-tailed. Given the a priori hypotheses, significant interactions were followed up with two planned $t$ tests using the adjusted means - independent PTSD versus independent no PTSD; interdependent PTSD versus interdependent no PTSD.

\section{Mental defeat.}

Examples of mental defeat were "I thought my life was over" (rating 2) (interdependent no PTSD) and "it looks like my time is up" (rating 2) (interdependnet no PTSD). Whereas an example of an autonomous frame of mind was "I knew I could help them and support them. I was powerful" (interdependent PTSD) (rating -2). Figure 8.1 shows the means for mental defeat. Independent culture had significantly greater mental defeat than interdependent culture, overall $F(1,98)=2.86, p=.045$ (one-tailed). Those with PTSD had greater mental defeat than those without PTSD, $F$ $(1,98)=8.09, p<.01$. The interaction between culture and PTSD status was significant, $F(1,98)=13.93, p<.01$. Independent PTSD had significantly greater mental defeat than independent without PTSD, $t(55)=5.13, p<.01, C I_{.95}=.74,1.70$, $d=1.36$. However, interdependent PTSD did not differ significantly from interdependent no PTSD, $t(47)=.43, p=.67, C I_{.95}=-.40, .62, d=.12$. 


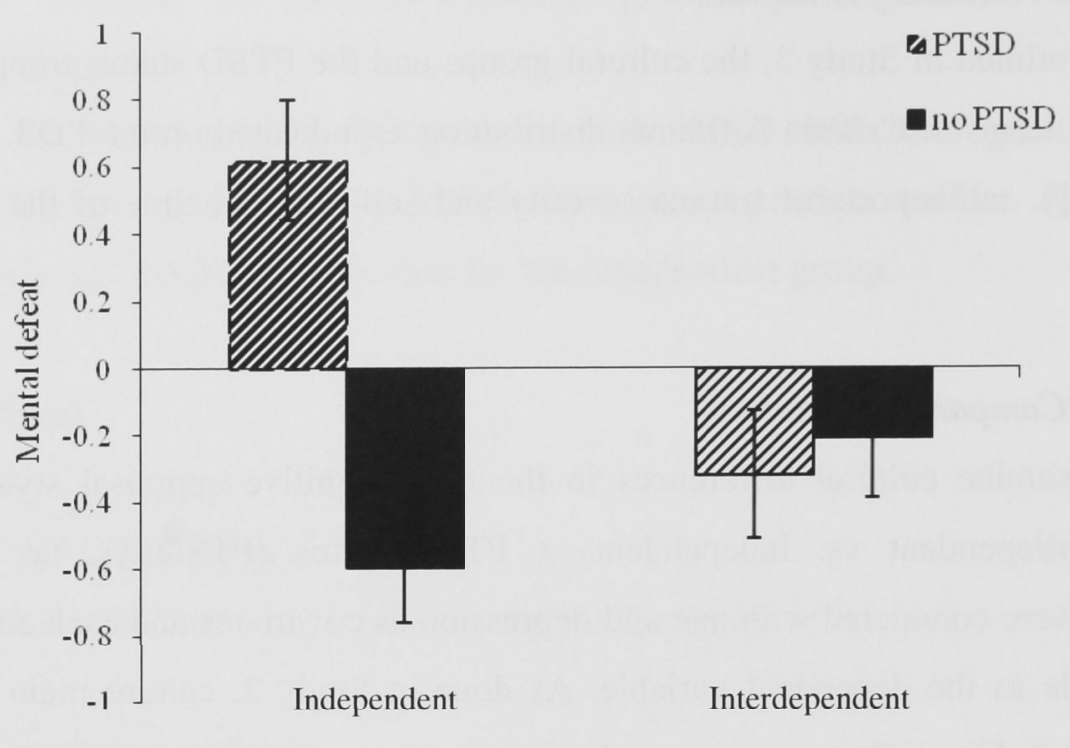

Culture Group

Figure 8.1 Adjusted means of mental defeat for independent and interdependent groups with and without PTSD. Error bars indicate \pm 1 SE of the mean.

\section{Control strategies.}

Examples of control strategies were "He [husband] stabbed me below the eye. I resisted and screamed as blood ran down my face onto my dress...so I left him" (rating 4) (interdependent no PTSD), "I felt that if I am an adult I may stop the man from beating the woman. I also felt I could kill the man" (interdependent no PTSD) (rating 4), and "I realised I had to become a better person...I started seeking God...exercised more, worked harder in my studies...sought to help others", (rating 3) (independent no PTSD). Figure 8.2 shows the means for control strategies. There was no significant difference between interdependent culture and independent cultures in control strategies, $F(1,98)=1.00, p=.16$ (one-tailed). Those with PTSD had significantly less control strategies than those without PTSD, $F(1,98)=4.76, p=$ .03. The interaction between culture and PTSD status was significant, $F(1,98)=$ $10.81, p<.01$. Independent PTSD had significantly fewer control strategies than independent without PTSD, $t(55)=4.49, p<.01, C I_{.95}=-1.61,-.61, d=1.20$. However, interdependent PTSD did not differ significantly from interdependent no PTSD, $t(47)=.74, p=.46, C I_{.95}=-.90, .42, d=.21$. 


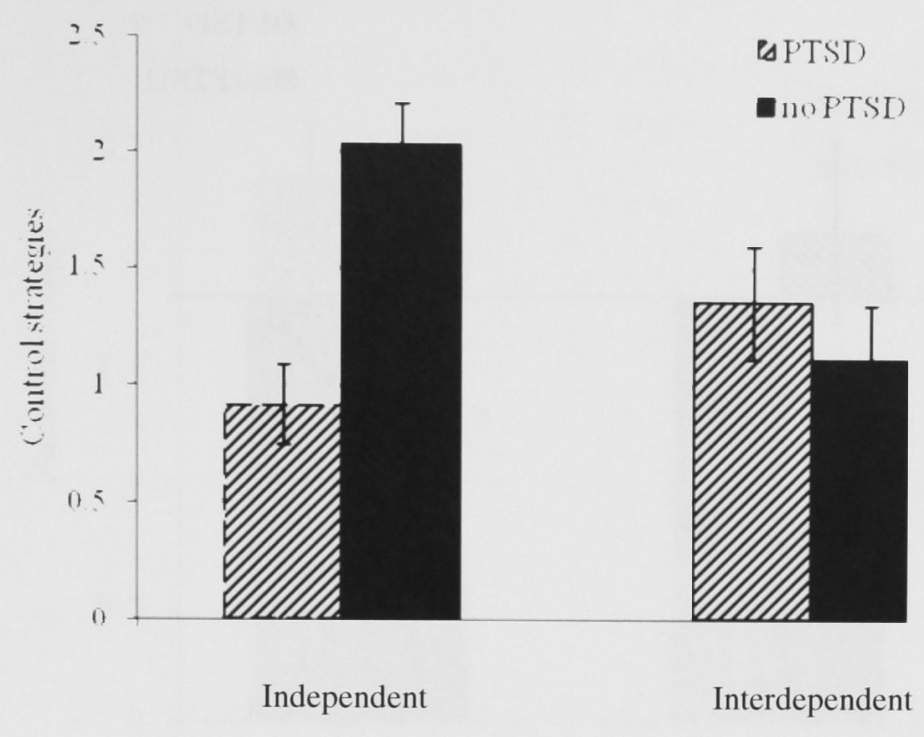

Culture Group

Figure 8.2. Adjusted means for control strategies of independent and interdependent culture group with and without PTSD. Error bars indicate \pm 1 SE of the mean.

\section{Alienation.}

Examples of alienation included "I feel betrayed, unloved and unsupported by my family" (rating 2) (independent PTSD), and "I was ignored by others....disregarded completely" (rating 2) (interdependent no PTSD). Examples of feeling socially supported included "I spent time with a group of friends" (rating -2) (independent no PTSD), and "as a result of her death - the time family spends together/relationships became so much more important with that particular family...you are thankful for those special people around you" (rating -2) (independent no PTSD). Figure 8.3 shows the means for alienation. There was no significant difference between interdependent cultures and independent cultures in alienation, $F(1,98)=.06, p=.40$ (one-tailed). Those with PTSD had significantly more alienation appraisals in their memories than did those without PTSD, $F(1,98)=$ $12.54, p<.01$. The interaction between culture and PTSD status was not significant, $F$ $(1,98)=.02, p=.88$. 


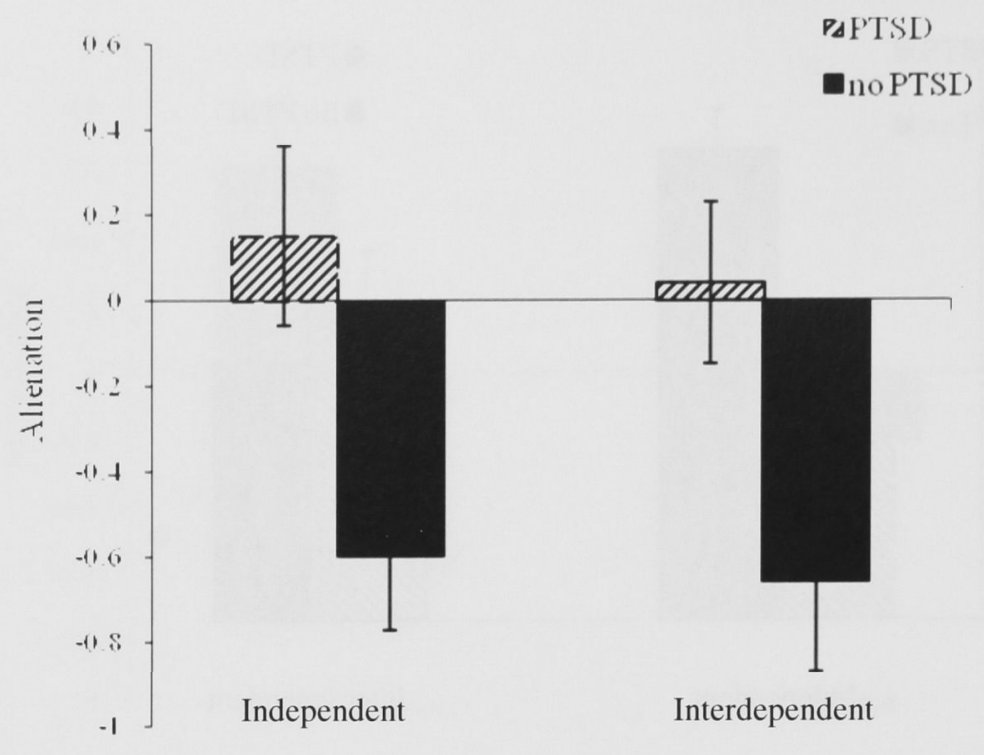

Culture Group

Figure 8.3 Adjusted means for alienation of independent and interdependent groups with and without PTSD. Error bars indicate \pm 1 SE of the mean.

\section{Permanent change.}

Examples of statements indicating permanent change included "The person I was ended that night...I have become an ugly burnt freak" (rating 4) (independent PTSD), and "My personality was adversely affected" (rating 3) (interdependent PTSD). Figure 8.4 shows the means for permanent change. Independent culture tended to have more permanent change appraisals than interdependent culture, $F$ (1, $98)=2.46, p=.06$ (one-tailed). Those with PTSD had significantly more permanent change appraisals than those without PTSD, $F(1,98)=28.05, p<.01$. The interaction between culture and PTSD status was significant, $F(1,98)=12.87, p<$ .01. Independent PTSD had significantly greater permanent change than independent without PTSD $, t(55)=7.36, p<.01, C I_{.95}=.1 .30,2.27, d=1.91$. Furthermore, interdependent PTSD did not differ significantly from interdependent no PTSD, $t(47)$ $=1.59, p=.12, C I_{.95}=-1.00, .12, d=.45$. 


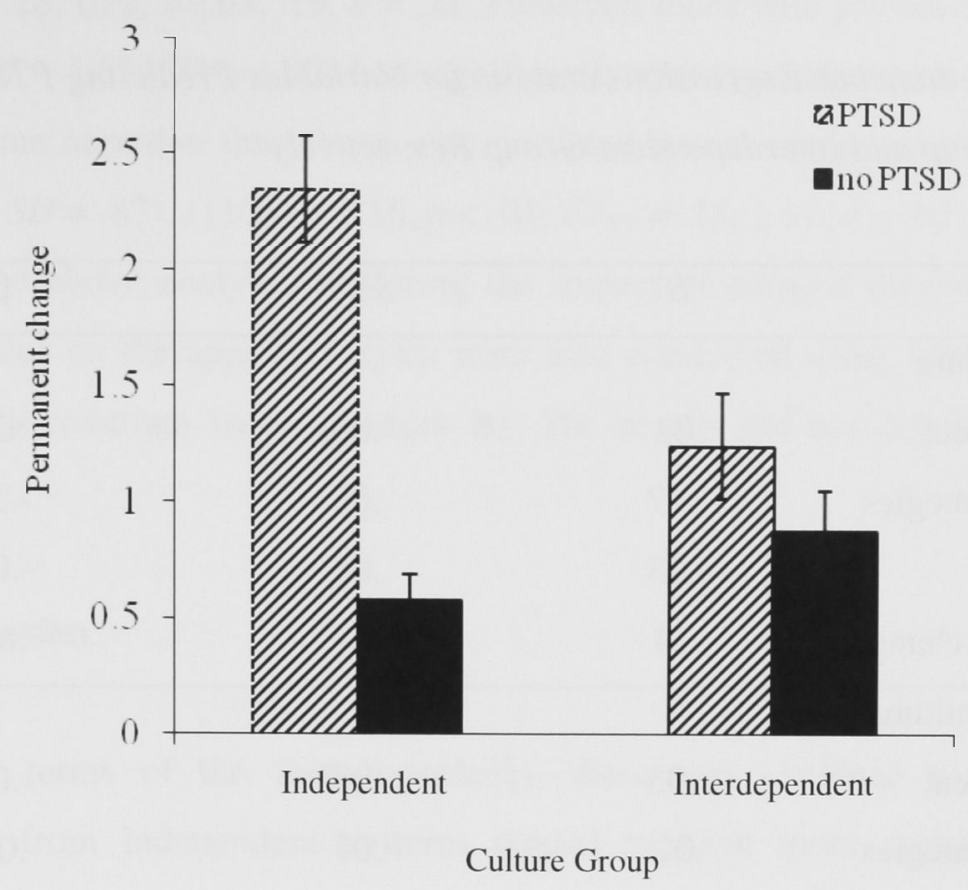

Figure 8.4 Adjusted means for permanent change of independent and interdependent group with and without PTSD. Error bars indicate \pm 1 SE of the mean.

\subsubsection{Simultaneous Regression Analyses}

Independent simultaneous regressions were conducted to examine the variables that predict PTSD (PDS score was used as the criterion measure) in the independent and interdependent groups. On the premise that mental defeat, control strategies, alienation and permanent change may influence PTSD development and maintenance (Dunmore et al., 2001) these score were used as predictor variables (Table 8.1). For independent culture a significant model emerged, $F(4,56)=22.10, p$ $<.01$, Adjusted $R^{2}=.60$. Control strategies $(t=3.31, p<.01)$ and permanent change $(t=5.80, p<.01)$ were significant predictors of PTSD. However, for the interdependent group a significant model did not emerge, $F(4,48)=1.98, p=.32$, Adjusted $R^{2}=.08$. Despite a significant model not emerging, it is worth noting that alienation did predict PTSD $(t=2.21, p=.03)$. However, such a finding must be considered with caution given the overall model was not strong. 
Table 8.1

Summary of Simultaneous Regression Analysis for Variables Predicting PTSD for the Independent Group and Interdependent Group Respectively

\begin{tabular}{lccc}
\hline Variable & $B$ & $S E B$ & $\beta$ \\
\hline Independent culture & .03 & .06 & .06 \\
Mental defeat & -.17 & .05 & -.36 \\
Control strategies & -.03 & .05 & -.06 \\
Alienation & .24 & .04 & .61 \\
Permanent change & & & \\
\hline Interdependent culture & -.03 & .08 & -.06 \\
Mental defeat & .02 & .07 & .05 \\
Control strategies & .15 & .07 & .31 \\
Alienation & .09 & .07 & .17 \\
Permanent change & & & \\
\hline
\end{tabular}

Note. $R^{2}=.63$ for independent culture model. $R^{2}=.15$ for interdependent culture model.

\subsubsection{Trauma Type (interpersonal versus non-interpersonal)}

Given that Ehlers' (e.g., Ehlers et al., 2000) work has explored these cognitive appraisal styles in survivors of interpersonal traumas, and it is uncertain whether these cognitive styles are moderated by trauma type, each memory was coded as interpersonal (i.e., rape, assault, combat, torture, assault) or not (accident, natural disaster, illness, other). It was found that the independent (50.88\%) and interdependent $(57.14 \%)$ groups did not differ in terms of the number of interpersonal trauma memory retrieved, $\chi^{2}(1, N=106)=.42, p=.52$. However, it was found that those with PTSD $(68.00 \%)$ retrieved more interpersonal traumas than those without $\operatorname{PTSD}(41.07 \%), \chi^{2}(1, N=106)=7.71, p<.01$.

Independent $t$ tests were conducted to compare interpersonal trauma type versus non-interpersonal trauma type using the four cognitive appraisal styles as dependent variables. Interpersonal and non-interpersonal trauma types did not differ in terms of mental defeat $(M=-.06, S D=1.04 ; M=-.28, S D=.81$, respectively $), t$ $(104)=1.20, p=.23, C I_{.95}=-.58, .14, d=.24$, control strategies $(M=1.34, S D=$ $1.15 ; M=1.49, S D=1.06$, respectively), $t(104)=.69, p=.49, C I_{.95}=-.28, .58, d=$ .14 , or alienation $(M=-.18, S D=1.02 ; M=-.40, S D=1.08$, respectively $), t(104)=$ 
$1.08, p=.28, C I_{.95}=-.63, .19, d=.21$. However, those who provided an interpersonal trauma $(M=1.57, S D=1.27)$ had significantly more permanent change appraisals in their trauma narrative than those who provided a non-interpersonal trauma memory $(M=.89, S D=.87), t(104)=3.16, p<.01, C I_{.95}=.25,1.11, d=.62$.

The above analyses exploring the impact of cultural differences in self and PTSD status on the appraisal styles were also conducted using interpersonal trauma status as a covariate (see Appendix B). The results did not differ from the above analyses.

\subsection{Discussion}

In terms of the first hypothesis, there was evidence to suggest trauma survivors from independent cultures tended to have more negative autonomous cognitive appraisal styles (mental defeat and permanent change strategies [control strategies only tended to be greater in the independent group than interdependent group]) in their trauma narratives than trauma survivors from interdependent cultures. This contradicts the hypothesis and findings of Study 2, which suggested a lack of cultural differences in the cognitive appraisal styles (mental defeat, control strategies) utilized during trauma, while suggesting cultural differences would be only evident in the cognitive appraisal styles (alienation, permanent change) generated post-trauma. The demarcation between appraisals being generated during and following trauma is not so clear. Rather, previous findings (e.g., Mesquita \& Walker, 2003) indicating cultural variation in self impacts on everyday appraisals, applies equally to traumarelated appraisals. Specifically, even in trauma appraisals, independent cultures appraise situations, events and life encounters in terms of personal control and autonomy, while in interdependent cultures personal control and autonomy has very limited applicability. However, research (e.g., Mesquita \& Walker, 2003) indicates that, unlike independent cultures, interdependent cultures emphasize interdependence in their appraisals. Yet, there were no cultural differences between independent and interdependent cultures in terms of alienation. This unexpected finding is discussed further below.

Overall, the results indicated that cultural differences in self moderate the relationship between cognitive appraisal styles and psychological adjustment. In terms of the autonomous cognitive appraisal styles, those from independent cultures with 
PTSD had more mental defeat and permanent change appraisals and less control strategies in their trauma memories than those from independent cultures without PTSD. However, as predicted trauma survivors with and without PTSD from interdependent culture did not differ in terms of mental defeat, control strategies or permanent change appraisals. In terms of relatedness cognitive appraisals, alienation differentiated between trauma survivors with and without PTSD from both cultural groups. That is, for both interdependent and independent cultures those with PTSD had more alienation appraisals in their trauma narratives than did those without PTSD. Unexpectedly, this relationship was not significantly more marked in the interdependent culture group when compared to the independent culture group.

For the independent culture group, the regression analyses revealed that using the four cognitive appraisal styles as predictor variables of PTSD resulted in a significant model emerging. Control strategies and permanent change appraisals were unique predictors of PTSD for the independent culture group. In contrast, for the interdependent culture group the regression analyses, using the four cognitive appraisal styles as predictor variables of PTSD, did not produce a significant model, although alienation was the only unique predictor of PTSD for this group.

These results are consistent with those of other studies (e.g., Ehlers et al., 2000) that show that these cognitive variables are related to PTSD. They also demonstrate, however, that the relationship between the cognitive variables and PTSD is moderated by cultural variation in self. Specifically, the findings indicate that autonomous and relatedness appraisals are instrumental in the etiology and maintenance of PTSD for those with an independent self-construal while only relatedness appraisals appear instrumental in the etiology and maintenance of PTSD for those with an interdependent self-construal.

It must be noted that alienation in the independent group, although not a unique predictor of PTSD, also distinguished between those with and without PTSD. It was initially thought that alienation may not be a single construct (i.e., there needs to be consideration of who the trauma survivor feels alienated from, the context of alienation, the length of time of alienation, the influence of alienation on self, etc.). These possibilities were explored in the narratives but did not appear to be plausible explanations. Instead, it is proposed that alienation is a maintaining factor in both cultures, but via different means. Ehlers (e.g., Ehlers et al., 2000) suggests that alienation maintains PTSD because appraisals and feelings of alienation result in the 
PTSD sufferer not engaging with the world. Hence, the survivor fails to alter selfcognitions and "re-learn" that the world is safe and people are good. PTSD is maintained as current situations are perceived as threats, as the survivor has not altered their views of others and the world. This is an individualistic approach to alienation, as it is in relation to the private self and the individual shifting private cognitions and appraisals. It is proposed that alienation is maintaining PTSD in interdependent cultures in a different manner. That is, alienation challenges the relatedness self. The survivor feels isolated from the group and thus, perceives themself as failing as a socially valued relatedness being. Consequently, alienation impacts on the public (i.e., social roles) and communal (i.e., interpersonal/relatedness) aspects of self. This proposal is supported by Markus and Kitayama's (1991) assertion that for the independent self-construal "others, or the social situation in general, are important, but primarily as standards of reflected appraisal, or as sources that can verify and affirm the inner core of the self' (p. 224), whereas the interdependent selfconstrual may adhere to goals and motivations that are significantly shaped and dominated by the reaction of others and in doing so gain self-definition. They propose relationships for the independent self-construal may be viewed as a means for obtaining individual goals, whereas in interdependent self-construal, relationships will often be ends in and of themselves. Additionally, according to Sato (2001) in collectivistic societies, a person without a strong sense of relatedness is often considered a dysfunctional member of society and such societies are thus, relatively intolerant of a lack of relatedness in an individual. He claims that for emotional health, individuals holding an interdependent self-construal have a need to be accepted by others. Further research is required to investigate the mechanisms by which alienation is involved in the etiology and maintenance of PTSD and the impact of cultural variation in self on such mechanisms.

Theoretically, the findings support Mesquita and Walker's (2003) argument that cultural differences in self-construal moderates the relationship between the way in which individuals appraise situations, events and life encounters and affective responses. Specifically, research (e.g., Mesquita \& Karasawa, 2002) has demonstrated that while those from independent cultures tend to experience more positive affect when they appraise a situation as under personal control, for those from interdependent cultures personal control appraisals seem to have less impact on affective responses. Furthermore, the findings support Sato's (2001) claim that for 
those with an independent self-construal poor mental health results when personal control over their environment is perceived to have diminished, whereas for those with an interdependent self-construal poor mental health seems unrelated to the perceived level of personal control over the environment but rather may result when there is perceived alienation or isolation. However, the findings also suggest for those with an independent self-construal, PTSD can be associated with appraised alienation or isolation.

Additionally, these findings support Ehlers and Clark's (2000) appraisal model. The appraisal model theorizes that PTSD occurs only if "individuals process the traumatic event and/or its sequelae in a way which produces a sense of a serious current threat" (p. 320). The model proposes this threat can be, and very commonly is, a threat to self. The model suggests that once the sense of current threat is activated it is accompanied by PTSD symptoms. The findings of this study support the appraisal model in that they suggest that trauma appraisals that threaten the self (i.e., by negative autonomous appraisals and negative relatedness appraisal for those with an independent self-construal and by negative relatedness appraisals for those with an interdependent self-construal) may produce a sense of current threat which is accompanied by PTSD symptoms. However, the findings suggest the appraisal model may need to consider and make explicit how cultural differences in self impact on the way in which appraisals may be implicated in the etiology and maintenance of PTSD. Specifically, while the appraisal model posits that PTSD is the result of "individual differences in the appraisal of the trauma and/or its sequelae" (p. 320, italics added), it seems that this may need to be extended to include cultural differences in the appraisal of the trauma and/or its sequelae.

The findings have clinical implications as they suggest that although a clinical awareness of the impact of trauma on appraisals is essential, awareness also needs to consider cultural factors which moderate any impact. Specifically, assessment and therapy needs address the impact of trauma on appraisals associated with private aspects of self and the impact of trauma on appraisals associated with public and collective components of the self. This would include identifying, challenging and reappraising relatedness appraisals (e.g., I am isolated, I am alienated, I am not a good mother, I let others down, etc.). Similar to Study 3, these results support the growing advocacy (e.g., Schwartz, 2005; Tarrier \& Humphreys, 2003) for the use of interventions that target social support as adjuncts to cognitive behavior therapy. 
Ehlers (e.g., Ehlers, Clark, et al., 1998) has found that the level of these appraisals in trauma narratives impacts on treatment outcomes. In general, those with high levels of mental defeat, alienation and permanent change appraisals do not show the same therapeutic improvements following exposure treatment. It is suggested that these clients require a stronger focus on cognitive work alongside the exposure work. It is recommended that research explores whether culture moderates this relationship between the trauma appraisals and treatment outcomes.

Finally, as Ehlers' work has been solely conducted in populations who have endured severe, interpersonal traumas (such as rape and torture); one aim of this research was to explore if these appraisal styles are present following all trauma types or only exclusively after interpersonal severe traumas. This study controlled for time since trauma, trauma history and self-reported trauma severity. The culture and PTSD status groups were equally matched on all of these variables, and as in Ehlers' work the four cognitive appraisals were evident, differentiated between those with and without PTSD and predicted PTSD. The study compared the cognitive variables in those who had endured an interpersonal trauma (e.g., rape, torture, assault) and those who had not (e.g., natural disaster, serious accident, serious illness). There were no cultural differences in the number of people who retrieved an interpersonal and noninterpersonal trauma type but those with PTSD were more likely to retrieve an interpersonal trauma than a non-interpersonal trauma. Regardless of cultural group, it was found that those who had endured an interpersonal trauma had more permanent change appraisals than those who had not endured an interpersonal trauma. This is not surprising given interpersonal trauma may be perceived to target the individual and invades the self whereas non-interpersonal trauma may be perceived more as the result of chance, a random occurrence and more distant from the self. All other cognitive appraisal variables were comparable across these groups. Furthermore, when using interpersonal trauma classification as an additional covariate, the same results emerged.

A limitation of this study was in the method of PTSD diagnosis. Second, retrieving memories in English may impact on how appraisals are presented. Third, there is an acknowledgement of possible demographic differences between groups which may have confounded the cultural independent variable. While there is little evidence that such factors would affect trauma appraisals it may be helpful for groups to be more closely matched in further research. Finally, it would be useful in future 
studies to examine the above, matching trauma type (i.e., just rape survivors, just torture survivors, etc.), as is done in Ehlers' (e.g., Ehlers et al., 2000) work.

Despite the shortcomings, this study demonstrated a factor that may be maintaining PTSD in interdependent populations. The study replicated Ehlers' (Ehlers et al., 2000) findings; cognitive appraisal styles (mental defeat, control strategies, alienation and permanent change) distinguish between those with PTSD from those without PTSD. However, these findings appear culturally specific, that is, these differences were only found in the independent sample except alienation. Unlike the independent cultural group, autonomous cognitive appraisals did not differentiate between those with and without PTSD from interdependent cultures. However, like the independent group, alienation did differ between those with and without PTSD from interdependent cultures. Therefore, the only cognitive appraisal style distinguishing between those with and without PTSD and predicting PTSD in interdependent cultures was alienation, a relatedness cognitive appraisal. 


\section{CHAPTER 9}

\section{Study 5: Cultural Differences in Meanings Attached to Trauma Autobiographical Memory in Posttraumatic Stress Disorder}

Study 5 is a brief supplementary study that provides a preliminary investigation of the impact of trauma on meaning and belief systems. A thorough examination of the impact of cultural variation in self on the impact of trauma on belief systems is beyond the breadth of this thesis, and is a thesis topic, in and of it self. Socio-cognitive theories of PTSD (e.g., Dalgleish, 2004; Ehlers \& Clark, 2000; Horowitz, 1986, 1997; Janoff-Bulman, 1992) propose that trauma impacts on survivors' belief systems and suggest mechanisms regarding the adjustment required to reconcile the traumatic event with prior beliefs and expectations (Resick \& Calhoun, 2001). The models posit that assumptions originally held about the self, others and world are shattered by trauma resulting in a transformation in meaning (Dalgleish, 2004; Janoff-Bulman, 1992). Resick and Calhoun (2001) note that many socio-cognitive theorists propose that in the aftermath of trauma, survivors make a conscious effort to search for meaning, to ascribe cause or blame, and to resolve conflicts between the trauma and prior beliefs, in an aim to reduce negative emotion and restore a sense of control or autonomy over one's environment. However, the universality of this search for meaning is questioned. That is, it is questioned as to whether trauma survivors from interdependent cultures, cultures in which relatedness is valued and personal control and autonomy have less relevance, also embark on this same pursuit for meaning in order to restore a sense of control or autonomy.

Dalgleish (2004) asserts cognitive therapy targets the interpretation and meaning of the trauma memory. He claims that the intention of cognitive therapy is to "counteract posttrauma transformation of meaning" (p. 231), or as Resick and Calhoun (2001) state, the aim of treatment is to establish pre-trauma fundamental beliefs and reach a point of equilibrium. Ehlers and Clark (2000) suggest assessment include identifying trauma memory meaning and specific characteristics about the nature of the memory, and therapy consist of cognitive-restructuring of altered assumptions and beliefs.

It is evident that pre-trauma belief systems, transformation in meaning and mechanisms utilized in reconciling the trauma with prior beliefs are three fundamental components in understanding the development, maintenance and in turn, treatment of PTSD. However, the central thesis of Markus and Kitayama's (1991) theory is that the cultural distinction in self-construal governs many belief systems. They claim that for those from independent cultures, beliefs about self and others tend to emphasize the separateness of the 
individual, and of the individual being comprised of internal attributes. In these cultures, there is a belief that one is required to be unique, exercise agency, and influence others, objects and situations in a way in which one will achieve personal goals. In contrast, those from interdependent culture, beliefs about self and others are centered round connectedness and interdependence with others. There are strong beliefs that an individual is expected to establish and maintain harmony within social relationships. The socio-cognitive models of PTSD appear to have not considered the impact of cultural distinction in self on survivor's efforts in the aftermath of trauma to search for meaning, to ascribe cause or blame, and to resolve conflicts between the trauma and prior beliefs.

This final study attempts to explore how cultural differences in self impact on the meanings attached to the trauma autobiographical memory by asking participants what the memory means to them, and if aspects or features of the memory are more significant. It is proposed that, given Markus and Kitayma (1994) claim independent cultures tend to associate meaning with the self as a unique, independent, self-contained, autonomous individual, while interdependent cultures associate meaning with the self as an interdependent entity attending to and fitting in with others, trauma memory meanings of those from independent cultures will be related to independence, while trauma memory meanings of those from interdependent cultures will be associated with interdependence.

\subsection{Hypotheses}

It is predicted that those from independent cultures will focus on independence and the uniqueness of the individual, while interdependent cultures will focus on interdependence when attributing meaning to the trauma autobiographical memory.

\subsection{Method}

\subsubsection{Participants}

Participants were recruited as in Study 3.

\subsubsection{Measures}

Participants were asked after the narrative task, "What does this traumatic memory mean to you?" and "Are there any aspects or features of the traumatic memory that stand out 
or mean more to you?". Participants were given two lines per question to write down their responses.

\subsubsection{Design and Procedure}

Design and procedure are outlined in Study 3.

\subsection{Results}

\subsubsection{Group and Participant Characteristics}

Group and participant group characteristics are outlined in Study 3.

\subsubsection{Trauma Memory Meanings}

As shown in Table 9.1, participants' responses to what the trauma memory meant to them were grouped according to the following categories: change in views of self and life (such as self-blame, self-doubt, I am a bad person, self-reflection, it destroyed my life, etc.); emotion (such as emotional responses, e.g., fear, guilt, emotional numbing, and/or change in emotion); change in views of the world (such as the world is unjust and unpredictable, people are not nice, etc.); family importance (such as a realization of how important family is, impact of trauma on family and wanting to assist family); on-going symptoms; and nothing/over it.

To examine culturally dependent differences in trauma memory meaning a chi-square with culture (independent; interdependent) and trauma memory meaning (change in views of self and life; emotion; change in views of the world; family importance; on-going symptoms; nothing/over it) was used. Although, the $2 \times 6$ contingency table had two of its cells with frequencies less than five, the assumptions of chi-square were met as there was more than five respondents in $80 \%$ of cells (e.g., Hinkle, Wiersma, \& Jurs, 2003). The independent and interdependent cultural groups did differ in terms of trauma memory meaning distribution, $\chi^{2}$ $(5, N=106)=18.93, p<.01$. Post-hoc follow-up tests for cultural differences were conducted using chi-square with culture (independent; interdependent) and each trauma memory meaning (reported; not reported). In order to compensate for alpha inflation, Bonferroni's correction was used and $\alpha$ was set at .04. The cultural groups differed in terms of the following distributions; change in views of self and life, $\chi^{2}(1, N=106)=14.46, p<$ .01 , and family importance, $\chi^{2}(1, N=106)=5.07, p=.02$. However, the groups did not differ in terms of emotion, $\chi^{2}(1, N=106)=.42, p=.52$, change in world views, $\chi^{2}(1, N=$ 
106) $=.02, p=.89$, symptoms, $\chi^{2}(1, N=106)=3.52, p=.06$, and nothing, $\chi^{2}(1, N=106)=$ $.80, p=.37$.

To examine PTSD status differences in trauma memory meaning a chi-square with PTSD status (PTSD; PTSD) and trauma memory meaning (change in views of self and life; emotion; change in views of the world; family importance; on-going symptoms; nothing/over it) was used. The PTSD and no PTSD groups did not differ in terms of trauma memory meaning distribution, $\chi^{2}(5, N=106)=2.10, p=.84$.

Table 9.1

Percentage of Participants who attached the Following Meanings to the Trauma Memory.

$$
\text { Independent culture Interdependent culture }
$$

\begin{tabular}{lcccc}
\hline & PTSD & No PTSD & PTSD & No PTSD \\
Change in views of self/life & 50.00 & 48.38 & 20.83 & 8.00 \\
Emotion & 26.92 & 9.67 & 20.83 & 28.00 \\
Change in views of world & 15.38 & 22.58 & 12.50 & 28.00 \\
Family importance & 0.00 & 6.45 & 12.50 & 4.00 \\
Symptoms & 0.00 & 3.23 & 12.50 & 8.00 \\
Nothing & 7.69 & 9.67 & 12.50 & 16.00 \\
\hline
\end{tabular}

Participants' responses to distinct aspects of the trauma memory were grouped into the following categories; flashbacks of a particular detail; emotional response; changes in beliefs about the self; family concerns; changes in beliefs about others and the world; and nothing stands out. To examine culturally dependent differences in distinct aspects of the trauma memory a chi-square with culture (independent; interdependent) and distinct aspects of the memory (flashbacks; emotional response; changes in beliefs about the self; family concerns; changes in beliefs about others and the world; and nothing stands out) was conducted. The independent and interdependent cultural groups (flashbacks, 12.22\%, $12.42 \%$; emotional response, $24.75 \%, 12.25 \%$; self beliefs, $18.3 \%, 8.17 \%$; family concerns, $6.77 \%, 16.34 \%$; other/world beliefs, 20.60\%, 10.09\%; nothing, $17.37 \%$, 40.75\%, respectively) did not differ in terms of distinct aspects distribution, $\chi^{2}(5, N=106)=10.25, p$ 
$=.07$. To examine PTSD status differences in distinct aspects of the trauma memory a chisquare with PTSD status (PTSD; no PTSD) and distinct aspects of the memory (flashbacks; emotional response; changes in beliefs about the self; family concerns; changes in beliefs about others and the world; and nothing stands out) was conducted. The PTSD and no PTSD groups (flashbacks, 16.19\%, 8.45\%; emotional response, 19.71\%, 17.29\%; self beliefs, $17.63 \%$, 8.84\%; family concerns, $10.26 \%, 12.84 \%$; other/world beliefs, $9.78 \%, 20.91 \%$; nothing, $26.44 \%, 31.68 \%$, respectively) did not differ in terms of distinct aspects distribution, $\chi^{2}(5, N=106)=5.68, p=.34$.

\subsection{Discussion}

Study 5 found support for the prediction that cultural differences in self impacts on the meanings attached to the trauma autobiographical memory. It was predicted that those from independent cultures would focus on independence of the individual, while interdependent cultures would focus on interdependence when attributing meaning to the trauma autobiographical memory. This hypothesis was supported. For independent cultures trauma memory meaning was associated with self-change and life-change, while interdependent cultures tended to focus on interdependence as they attributed trauma memory meaning to a heightened awareness of family importance. Cultural groups did not differ in reports of aspects of the trauma autobiographical memory that were more significant.

This finding has theoretical implications. The socio-cognitive theories of PTSD (e.g., Dalgleish, 2004; Ehlers \& Clark, 2000) propose that trauma impacts on belief systems. However, it appears that belief systems differ culturally. Markus and Kitayama's (1991) position, that the cultural distinction in self impacts on beliefs systems and meaning of the self, others and world needs to be considered and made explicit when discussing prior belief systems, the notion of transformation in meaning (independent cultures may focus on transformation in meaning to the self, whilst interdependent cultures may giver greater focus to the family and interdependence within social groups), and the adjustment required to reconcile the traumatic event with prior beliefs and expectations in PTSD models.

The findings have clinical implications. Dalgleish (2004) suggests that cognitive therapy targets the interpretation and meaning of trauma memory and the effects of the trauma on lives. However, as has been seen in this study the meaning of the trauma memory appears to be impacted on by cultural differences in self. Adopting Ehlers and Clark's (2000) treatment framework, the study suggests assessment needs to be aware that those with an 
independent self-construal may focus on trauma memory meaning to the self and life, whilst interdependent culture may focus on trauma meaning to family and relational aspects of self.

A limitation of this study was the methodology. Participants were asked to write about what the trauma autobiographical memory meant to them. This may have been biased by what participants were willing to disclose and such a task was not an optimal measure of belief systems, meaning systems and transformation in meaning. However, the preliminary findings from this study suggest that cultural differences in self may impact on belief systems and world views, and trauma memory meaning. 


\section{CHAPTER 10 \\ General Discussion}

In this final chapter the main findings of this thesis are discussed in the context of the research questions defined in the introduction. Second, the socio-cognitive models of PTSD are critiqued in light of the findings. Third, a model that synthesizes the findings of this thesis with current theoretical understandings about the etiology and maintenance of PTSD and understandings about cultural variation in selfconstrual is developed. Fourth, the clinical implications of the findings are discussed. Finally, limitations and future directions are reviewed.

\subsection{Overview of Main Findings}

\subsubsection{Autobiographical Remembering of Trauma}

Studies 1 and 2 found, while the cultural distinction between autonomy versus relatedness was evident in autobiographical memories of everyday events, no cultural distinction was evident in the autobiographical remembering of trauma. The autobiographical remembering of trauma was predominately autonomous. Moreover, it was found that for those from independent cultures the level of autonomous orientation was similar in both the everyday and trauma memories, while those from interdependent cultures had higher levels of autonomous orientation in the trauma memory when compared to the everyday memory.

Study 3 found evidence to suggest that cultural distinction in self impacts on the relationship between the nature of autobiographical remembering and posttraumatic psychological adjustment. Trauma survivors from independent cultures with PTSD had less autonomous orientation in their autobiographical remembering than trauma survivors from independent cultures without PTSD. In contrast, trauma survivors from interdependent cultures with PTSD had more autonomous orientation in their autobiographical remembering than trauma survivors from interdependent cultures without PTSD.

\subsubsection{Integration of Autobiographical Memory of Trauma}

Studies 1 and 3 found that the cultural distinction in self moderated trauma's impact on personal identity, self-definition and psychological adjustment to trauma. 
Trauma-exposed participants from independent cultures with PTSD clearly displayed a more trauma-defined personal identity than those without PTSD. For those from interdependent cultures, however, there were no differences in trauma-centered identity between the PTSD and no PTSD groups.

\subsubsection{Appraisals}

Studies 2 and 4 found that the cultural distinction in self impacts on trauma appraisals. There was evidence suggesting trauma survivors from independent cultures had more negative autonomous cognitive appraisals in their trauma narratives than trauma survivors from interdependent cultures. However, no cultural differences in the number of negative relatedness cognitive appraisals were found. Moreover, Study 4 demonstrated cultural difference in self moderates the relationship between cognitive appraisal styles and psychological adjustment. Specifically, for independent cultures those with PTSD had more negative autonomous appraisals (more mental defeat and permanent change, and less control strategies) and more alienation appraisals in their trauma memories than those without PTSD. A number of these autonomous appraisals were unique predictors of PTSD. However, those with and without PTSD from interdependent cultures did not differ regarding autonomous cognitive appraisal styles but rather trauma survivors from interdependent cultures with PTSD had more alienation (relatedness) appraisals in their trauma narratives than trauma survivors from interdependent cultures without PTSD.

\subsubsection{Meaning and Belief Systems}

Study 5 found that cultural variation in self impacts on the meanings attached to the trauma autobiographical memory; trauma survivors from independent cultures focused on self-change and life-change, while trauma survivors from interdependent cultures focused on interdependence.

\subsection{Theoretical Implications}

Throughout this thesis it has been questioned as to whether current models of PTSD are culturally flexible enough to accommodate cultural variation in self. Based on the findings, the answer seems to be both yes and no. That is, the models, as they stand, account for most of observed phenomena associated with PTSD, and seem to 
be able to differentiate between those with and without PTSD. However, it is suggested, that the models be expanded to explicitly consider cultural variations in self. The PTSD models will now be critiqued in light of the findings.

\subsubsection{Self-Memory System (SMS)}

\section{Support.}

The findings support SMS (Conway, 2005) in its suggestion that the working self is the prime regulator of autobiographical retrieval. An example of such support is derived from the finding of cultural similarities in the autobiographical remembering of trauma, suggesting that how a person remembers a traumatic event is predominantly guided by an autonomous working self, regardless of the dominant goal orientation of their culture. Second, the introduction of the conceptual self, the role of the conceptual self in autobiographical remembering, and its relationship with the working self, outlined in the most recent modifications to SMS (Conway, 2005), appear equally appropriate and present in the autobiographical remembering in both cultural groups. Specifically, autobiographical remembering of everyday events seems to reflect the conceptual self. Third, SMS' prediction that the conceptual self can be altered to center on trauma in the aftermath of trauma and that self-coherence can be instrumental in this self-change, was supported. Finally, the results accord with the proposal derived from SMS; namely that change in the conceptual self is based on goals consistent with the existing goals of the conceptual self. Specifically, change in the conceptual self can occur when goals activated by the trauma match existing goals of the conceptual self.

\section{Further considerations.}

Although most of the findings support SMS, the findings do suggest SMS may be limited in its understandings of the self and in particular cultural variation in self. First, it is suggested that SMS consider the finding that cultural variation in self impacts on the relationship between the nature of the trauma memory and psychological adjustment. That is, for those with poorer psychological adjustment the conceptual self seemed to be challenged by the nature of the trauma memory (i.e., the level of autonomous orientation in the trauma memory or, in other words, the strength of the autonomous goal hierarchy activated to encode the trauma memory). It seems that the level of autonomous orientation in the trauma memory impacts on autobiographical remembering, the self, and the unique relationship between the two. 
Second, the thesis highlighted that the trauma memory alone is not sufficient in differentiating between those with and without PTSD and thus, suggests SMS give greater emphasis to components such as appraisals and the notion of transformation of meaning when accounting for the etiology and maintenance of PTSD. Finally, the findings suggest that SMS' proposal, that following trauma alteration in the conceptual self to center on trauma driven by a need for self-coherence, is moderated by culture. Specifically, the impact of cultural distinction in self was found to impact on the degree to which trauma becomes a central component to self-conception. SMS seems to have stopped short of considering and making explicit the impact of cultural variability in self-consistency needs.

\subsubsection{Centrality of Trauma Approach}

\section{Support.}

The findings accord with the centrality of trauma approach (Berntsen \& Rubin, 2006, 2007), which states following trauma, the social role of trauma survivor/victim can become central to personal identity. Berntsen and Rubin suggest, that self-change can be driven by a need for self-consistency and self-coherence, and thus, as in the case for SMS, the findings supported that the need for self-consistency and selfcoherence seems active in the move towards trauma-centered self-definition. Second, the results accord with the proposal derived from the centrality of trauma model that the social role of trauma victim/survivor is a culturally sanctioned role in independent cultures, given its alliance with cultural expectations of the self, personal identity and the individual life story. However, in interdependent cultures the social role of trauma survivor/victim may be viewed as culturally inappropriate, as it contradicts cultural expectations of a communal self. Finally, the results supported the proposal that the trauma memory can become the central cognitive reference point for other autobiographical memories.

\section{Further considerations.}

The findings suggest that the centrality of trauma model may be limited in terms of current understandings of impact of cultural distinction in self on the degree to which trauma becomes a central component to personal identity. The centrality of trauma approach seems not to have considered the impact of cultural variability in self-consistency needs. The findings of this thesis point to important cultural considerations both in the etiology of trauma-centered personal identity in PTSD and 
in its maintenance, which are not part of this psychological model of PTSD. Second, Berntsen and Rubin (2007, p. 221) state that "salient personal memories form turning points" and "if a trauma memory is seen as a central turning point in our life story it would also most likely be regarded as a central component of our personal identity". However, the results suggest that the trauma memory can become a cognitive reference point for the organization of other autobiographical memories without becoming the salient and important component of identity. Hence, alterations in identity are not necessarily the consequence of the trauma memory becoming a salient personal memory. Rather, alterations in identity seem dependent on the need for selfconsistency. It is recommended that their approach clarify this distinction; trauma memory centrality does not always result in identity change, especially in interdependent cultures.

\subsubsection{Appraisal Model}

\section{Support.}

There was considerable support for the appraisal model (Ehlers \& Clark, 2000). First, the findings accorded with the appraisal model's position that PTSD is the result of both the disturbance and dysregulation in autobiographical memory and individuals' processing trauma in a way that has excessive negative appraisals of the trauma and/or its sequelae. That is, the findings suggest that the nature of the trauma memory alone was not the only factor differentiating between those with and without PTSD. While autobiographical remembering for trauma appeared relatively culturally similar, the results indicated cultural variation in self impacts on the relationship between trauma appraisals and psychological adjustment. Supporting the appraisal model, it does seem that as a result of negative appraisals, individuals process the past threat as an on-going threat, in this case an internal threat to the conceptual self. Hence, future situations are appraised as dangerous. Second, the model allows for the thesis' assertion that PTSD may be maintained in independent populations by the information and memories the survivor retrieves following trauma aligning with negative autonomous appraisals of inadequacy and an inability to cope. This results in survivors continuing to view themselves as incapable, unworthy and failing as an autonomous person. However, in the interdependent group there was no evidence suggesting a relationship between autonomous cognitive appraisal styles and problematic psychological adjustment, as these autonomous appraisals seem not to 
challenge the interdependent self. For interdependent populations, PTSD may be maintained by appraisals of alienation, as the information and memories the survivor retrieves following trauma aligns with these appraisals of alienation, resulting in perceptions of the self as isolated and failing as an interdependent person. Although, alienation also differentiated between those with and without PTSD from independent cultures, it is proposed that alienation operates differently in maintaining PTSD in interdependent and independent cultures. This will be discussed below.

\section{Further considerations.}

The shortcoming of the appraisal model was that although there is a consideration of individual differences, cultural differences in memory and appraisals are ignored. Given this thesis found support for cultural variations in self impacting on the relationship between appraisals and psychological adjustment, it seems necessary to consider culture in the appraisal model. Furthermore, as stated above, the appraisal model posits that memory and appraisals are involved in the development and maintenance of PTSD. The memory component of their model is based on SMS. However, it is based on SMS presented in Conway and Pleydell-Pearce's paper published in 2000. In 2005, Conway introduced the conceptual self into the SMS, making it more sympathetic to cultural considerations. It is suggested the appraisal model make similar introductions. Finally, many of the suggested appraisals are quite individualistically focused. This is appropriate for autonomous appraisals. Few of Ehlers and Clark's identified appraisals focus on social roles. Additionally, even the communal/relatedness appraisals are predominately private self focused. For example, Ehlers (e.g., Ehlers et al., 2000) describes a relatively individualistic approach to alienation and refers to alienation as an obstruction to trauma survivors shifting private cognitions and appraisals. It is proposed that alienation may be maintaining PTSD in interdependent cultures by challenging the interdependent self. Hence, public and communal appraisals need to be given greater emphasis in the appraisal model.

\subsubsection{Transformation of Meaning Models}

\section{Support.}

The thesis did not thoroughly examine the socio-cognitive theories of PTSD (e.g., Dalgleish, 2004; Ehlers \& Clark, 2000; Janoff-Bulman, 1992) proposal that 
trauma impacts on belief systems and transformation in meaning. Hence, little support emerged from this thesis in support of such proposals.

\section{Further considerations.}

The preliminary findings, however, did suggest the models need to consider and make explicit that belief systems differ culturally. Eagle (2005) argues although the main assumptions about the world and self presented in PTSD models may be universal, the substance of these premises may differ culturally. Markus and Kitayama (1991) assert the cultural distinction in self impacts on beliefs, thoughts, feelings and behavior regarding self, others and world. Such positions needs to be considered and made explicit when discussing prior belief systems in PTSD models. The notion of transformation in meaning also needs to consider cultural differences. It seems independent cultures focus on transformation in meaning to the self, whilst interdependent cultures may giver greater focus to the family and interdependence. Resick and Calhoun (2001) propose that in the aftermath of trauma survivors search for meaning, ascribe cause or blame, and resolve conflicts between the trauma and prior beliefs, in an aim to restore a sense of autonomy. Although, independent cultures may aim to obtain autonomy, interdependent cultures may attribute meaning to other factors such as interdependence. Personal control and autonomy are less applicable in interdependent cultures. In sum, it is suggested that socio-cognitive theories of PTSD need consider cultural differences in belief systems, in assumptions about the self, others and world and how (and even if) trauma shatters these assumptions, and the adjustment required to reconcile the traumatic event with prior beliefs and expectations.

\subsubsection{Summary}

Overall, the findings point to important cultural considerations both in the etiology of PTSD and in its maintenance, which are not part of current psychological models of PTSD. The results of this thesis challenge these models to articulate how the cultural self aligns with their accounts. This reflection may result in alterations in structural aspects of PTSD models and in aspects/processes thought to be implicated in the maintenance of PTSD symptoms. It is thought, confirmed by the findings in this thesis, that PTSD models need to be more explicit regarding the impact of the cultural self on the processes involved in development and maintenance of PTSD. 


\section{3 'Threat to Conceptual Self (TCS)' Model}

The thesis has now reached the point in which it is appropriate to synthesize the findings with current theoretical knowledge about the etiology and maintenance of PTSD, current theoretical knowledge about cultural differences in self-construal and the conceptual framework outlined in Chapter 4, to produce a working model that offers a method of making sense of the findings and accounts for the relationship between trauma and the conceptual self in the etiology and maintenance of PTSD. This model has been depicted in Figure 10.1. 


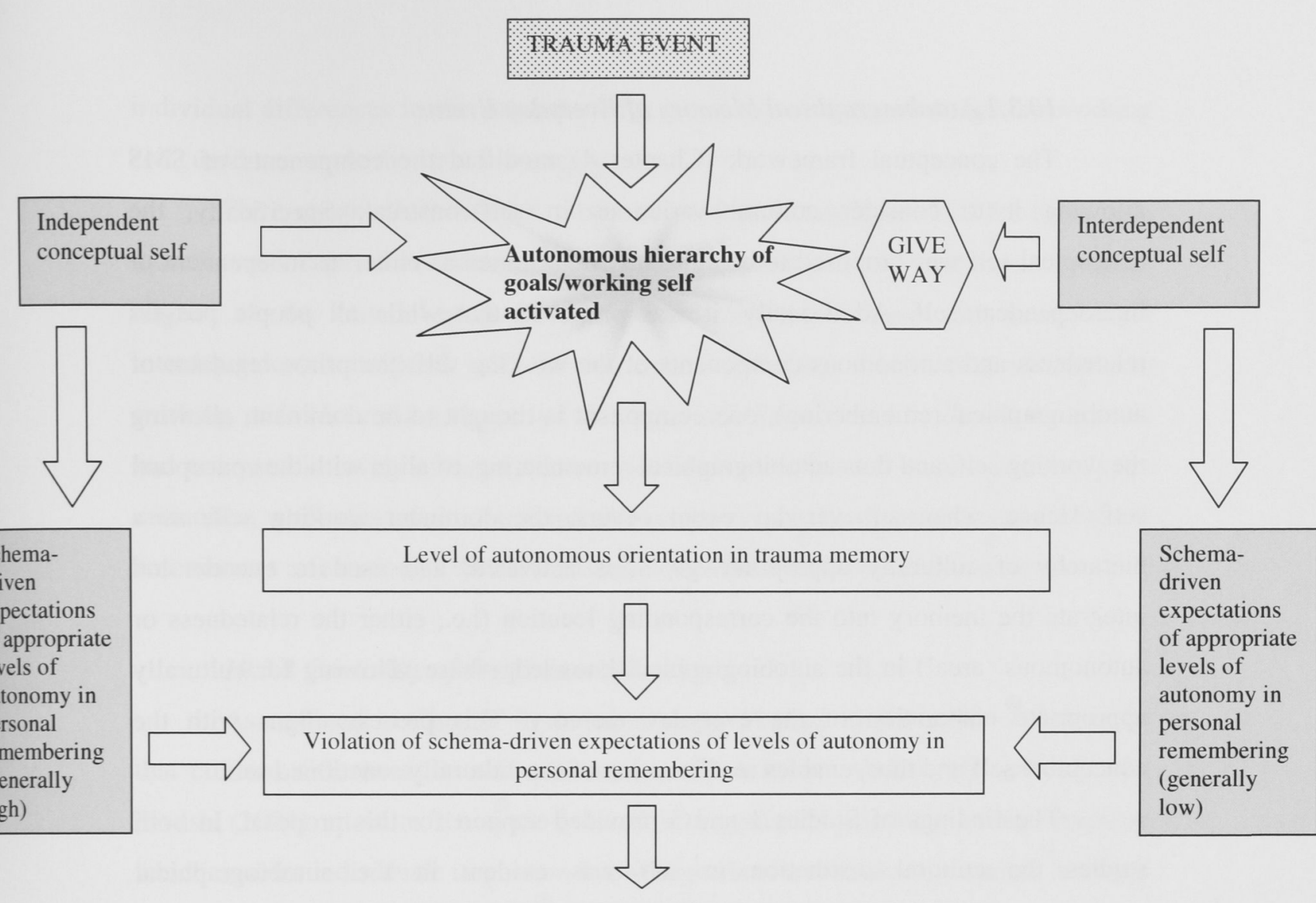

Trauma memory salient and the cognitive reference point for the organization of other autobiographical memories

Impact on the

development, expression

and maintenance of

conceptual self

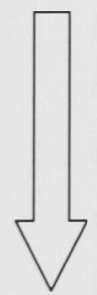

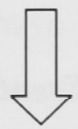

Need for selfconsistency \& self-coherence

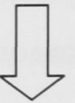

Trauma central to personal identity, self-concept and selfdefinition<smiles>C1=CCCCC1</smiles>

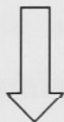

Negative Appraisals

Autonomous

Relatedness

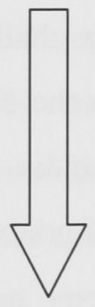

Meaning and belief systems altered transformation in meaning

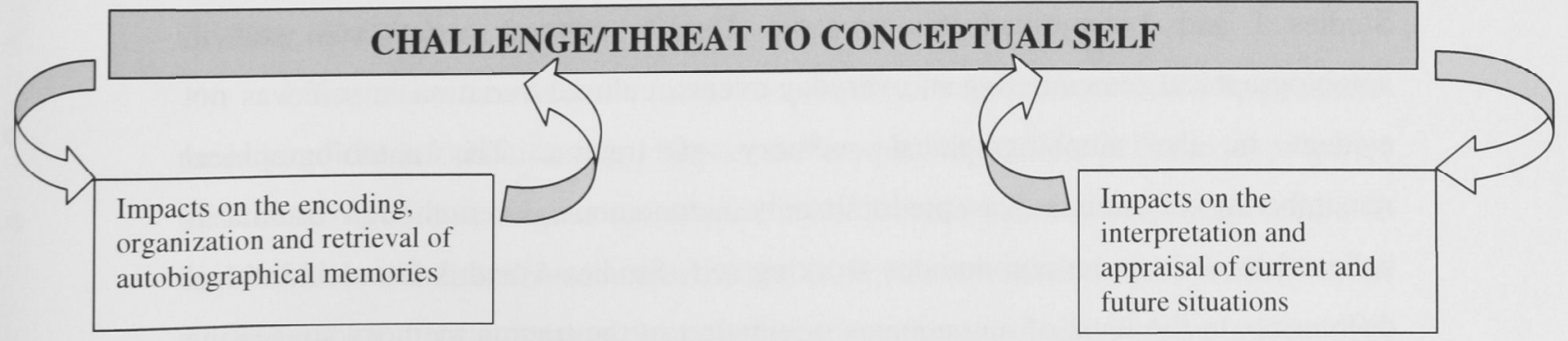

Figure 10.1 Threat to Conceptual Self(TCS) Model 204 


\subsubsection{Autobiographical Memory of Everyday Events}

The conceptual framework (Chapter 4) modified the components of SMS allowing it to consider cultural variations in self-construal. Specifically, the conceptual self was proposed to be culturally determined as either an independent or interdependent self. Additionally, it was proposed that while all people possess relatedness and autonomous components of the working self (the prime regulator of autobiographical remembering), one component is thought to be dominant, allowing the working self, and thus autobiographical remembering, to align with the conceptual self. Hence, when an everyday event occurs, the dominant working self, as a hierarchy of culturally appropriate goals, is activated, and used to encode and integrate the memory into the corresponding location (i.e., either the relatedness or autonomous 'area') in the autobiographical knowledge base, allowing for culturally appropriate elaboration of the everyday memory. This process aligns with the conceptual self and thus, enables reaffirmation of the culturally sanctioned self.

The findings of Studies 1 and 3 provided support for this proposal. In both studies the cultural distinction in self was evident in the autobiographical remembering of everyday and self-defining memories. The findings also suggested that autobiographical memories are elaborated in culturally appropriate ways.

\subsubsection{Autobiographical Memory of a Trauma Event}

In addition, the conceptual framework highlighted theorists' (e.g., Dalgeish, 2004) claims that trauma events have certain characteristics that challenge personal goals to survive, to protect personal safety, and to personally control and master the situation; that is, trauma challenges predominately autonomous goals. Therefore, it was proposed that given the culturally dominant self-construal can be overridden by temporary primes (Triandis et al., 1991), trauma, by its nature, activates an autonomous hierarchy of goals. Thus, the autobiographical remembering of trauma is predominately autonomous, regardless of the dominant self-system. The findings of Studies 1 and 3 supported this proposal. Despite cultural variation in self in autobiographical remembering of everyday events, cultural variation in self was not evident in the autobiographical memory of trauma. The autobiographical remembering of trauma was predominately autonomous. Even though trauma is believed to activate an autonomous working self, Studies 1 and 3 found individual differences in the level of autonomous orientation in the trauma memory suggesting 
individual differences in the strength of the hierarchy of autonomous goals (working self) used to encode the trauma memory.

The conceptual framework, proposed, as depicted in Figure 10.1 for those with a dominant independent conceptual self, the conceptual self and working self remain aligned in the remembering of trauma. However, for those with a dominant interdependent conceptual self, the conceptual self gives way to the autonomous working self. The results supported this proposal. Those from independent cultures had comparable levels of autonomous orientation in both their trauma and everyday memories, while those from interdependent cultures had significantly greater levels of autonomous orientation in their trauma memories than their everyday memories.

\subsubsection{Centrality of the Trauma Memory}

As outlined in Chapter 8, theorists' (e.g., Markus \& Kitayama, 1991) argue that cultural differences in self-construal govern schema. Additionally, Nelson and Fivush (2004) attest to the socio-cultural emergence of autobiographical memory which enables individuals to possess knowledge about culturally appropriate levels of autonomy in autobiographical remembering. Wang and Conway (2004) propose that such knowledge carries over into adulthood. By combining these stances, the TCS model suggests people have schema-driven expectations as to appropriate levels of autonomous orientation in autobiographical remembering derived from the conceptual self. Specifically, as Figure 10.1 shows, an independent conceptual self generally results in schema-driven expectations of high levels of autonomous orientation in autobiographical remembering, as high levels of autonomous orientation reaffirm personal control, mastery, uniqueness and autonomy (Wang \& Conway, 2004). In contrast, an interdependent conceptual self generally results in schema-driven expectations of low levels of autonomous orientation in autobiographical remembering, as autonomous orientation is not valued and has the potential to undermine group harmony, 'fitting in' and a sense of interdependence (Wang \& Conway, 2004).

Information that deviates from schema-driven expectations results in enhanced memory of the information (e.g., Brewer \& Treyens, 1981). The TCS model proposes that the level of autonomous orientation in the trauma memory has the potential to deviate from schema-driven expectations of levels of autonomous orientation in autobiographical remembering. Specifically, for trauma survivors with an independent 
conceptual self a low level of autonomous orientation in the autobiographical remembering of trauma and for trauma survivors with an interdependent conceptual self a high level of autonomous orientation in the autobiographical remembering of trauma may violate schema-driven expectations. Such a deviation from schema-driven expectations may result in an enhanced, highly accessible memory of the trauma event.

Berntsen and Rubin (1997) assert highly accessible autobiographical memories often become the reference points for the organization of autobiographical memories of less significant events. They suggest such memories structure "life narratives by providing turning points and by forming beginnings and ends of life time periods" (p. 418) in the autobiographical knowledge base of SMS (Conway \& Pleydell-Pearce, 2000). They propose that centrality of the trauma memory is associated with PTSD symptoms. Therefore, TCS proposes that one way, and not exclusively the only way, by which the trauma memory can become the reference point for other life events and thus associated with PTSD symptoms, is by the level of autonomous orientation in the trauma memory deviating from schema-driven expectations of levels of autonomous orientation. This proposal is supported by the findings (Study 3) that demonstrated that those from independent culture with PTSD had less autonomous orientation in their trauma autobiographical memory than those without PTSD, while those from interdependent culture with PTSD had more autonomous orientation in their trauma autobiographical memory than those without PTSD.

As Figure 10.1 demonstrates, the trauma memory, as the cognitive reference point for other autobiographical memories, can result in four potential outcomes; first, the trauma memory can impact on the functional role of autobiographical memory; second, the trauma memory can become central to identity, self-concept and selfdefinition; third, the trauma memory can moderate appraisals, and fourth, the trauma memory can impact on transformation of meaning. It is thought that all four potential outcomes threaten the conceptual self.

\subsubsection{Impact of Trauma Memory on Functional Role of Autobiographical}

\section{Memory}

The first potential outcome of centrality of the trauma memory is an impact of the trauma memory on the functional role of autobiographical memory. Centrality of 
the trauma memory in the organization of autobiographical memory means, according to Berntsen and Rubin (2007), that everyday events become associated with the trauma memory. Wang and Conway (2004) assert that autobiographical memories are instrumental in the development, expression and maintenance of the conceptual self. Therefore, as shown in Figure 10.1, the trauma memory, and its level of autonomous orientation, as central to autobiographical memory organization, is likely to impact on the expression, development and maintenance of the conceptual self. Consequently, the conceptual self is threatened. For those with an independent self-construal, low autonomous orientation in the central trauma memory means the conceptual self is developed, expressed and maintained in terms of low levels of autonomy. In contrast, for those with an interdependent self-construal, high autonomous orientation in the central trauma memory means the conceptual self is developed, expressed and maintained in terms of high levels of autonomy. Additionally, the trauma autobiographical memory is, what Pillemer (1998) coins, an anchoring event and "a lasting reminder of the way things are" (p. 74).Thus, the level of autonomous orientation in the trauma memory is likely to anchor the level of autonomous orientation in future everyday events and act as a lasting reminder of levels of autonomy in the self, again posing a potential threat to the conceptual self.

\subsubsection{Trauma Central to Identity}

A second potential outcome of the centrality of the trauma memory is that trauma becomes central to identity, self-concept and self-definition. The following theoretical assertions draw attention to the unique relationship between autobiographical memory and identity. Berntsen and Rubin (2007) propose that the way in which life stories, and thus autobiographical memories, are composed is closely related to self-understanding and identity. Pillemar (1998) suggests highly accessible autobiographical memories anchor and stabilize self-conceptions. McAdams (2001) argues that identity "takes the form of a story" (p. 101). Conway (2005) claims that autobiographical memory validates and supports the self, and the conceptual self is comprised of the life story. Wang and Conway (2004) assert that autobiographical memory is a constituent of identity and the self. Conway and Holmes (2004) suggest autobiographical memory defines the self. Therefore, the TCS model proposes centrality of the trauma autobiographical memory is most likely to be regarded as a central component of personal identity and the self. 
The TCS argues, however, as shown in Figure 10.1, the determining factor as to whether the trauma becomes central to identity and the conceptual self is the requirement for self-consistency. As stated throughout the thesis, theory and research (Suh, 2000, 2002) have demonstrated that self-consistency and self-coherence in identity is culturally variable. Therefore, if a trauma survivor has a requirement for self-consistency and is defined by a set of stable internal attributes, it is likely that a trauma memory, which is central to the life story and other autobiographical memories, becomes central to identity and the conceptual self. This results in a trauma-centered identity or trauma-centered conceptual self. Identity or conceptual self change will obviously threaten the conceptual self as it becomes centered round trauma.

\subsubsection{Appraisals}

A third potential outcome of the trauma memory as cognitive reference point for other autobiographical memories is that it results in negative on-going appraisals. Ehlers and Clark (2000) claim that there is a reciprocal relationship between the nature of the trauma memory and appraisals. The recall of the trauma memory may be biased by negative appraisals and in turn, information that is consistent with these appraisals is selectively retrieved from autobiographical memory. Therefore, if the trauma memory is central to autobiographical memory organization, it is likely that appraisals will reflect the nature of the trauma memory and have the potential to threaten the conceptual self. Low levels of autonomous orientation (i.e., little evidence of personal control, evaluations, mastery, uniqueness, etc.) in the trauma memory are likely to result in negative autonomous appraisals (e.g., "I cannot cope, I have no personal control, I am permanently changed", etc.). Such appraisals are thought to impact on the independent conceptual self but have a neutral impact on the interdependent conceptual self. In contrast, high levels of autonomous orientation (i.e., evidence of personal control, evaluations, mastery, uniqueness, etc.) in the trauma memory are likely to result in autonomous appraisals (e.g., "I can cope, I have control over the environment, I mastered the situation, I am unique", etc.). Appraisals of personal control, uniqueness and mastery may have a neutral or positive impact on

the independent conceptual self but have limited applicability to the interdependent conceptual self (Mesquita \& Walker, 2003). High levels of autonomous content in autobiographical remembering will over time result in negative relatedness appraisals 
as high levels of autonomy challenge the interdependent self and contradict interdependence, group harmony and adhering to social obligations.

\subsubsection{Meaning Systems}

The fourth potential outcome is that the centrality of the trauma memory in autobiographical memory challenges beliefs and meanings (i.e., transformation of meaning, Dalgleish, 2004). Cohen $(1989,1998)$ claims that autobiographical memory aids the development of opinions, beliefs and attitudes and Bluck et al. (2005) state that autobiographical memory anchor personal values, act as originating events for chosen life directions and as turning points that redirect the life path. Therefore, centrality of the trauma memory is likely to impact on belief systems. Given, the conceptual self is comprised of belief systems, alterations to beliefs and meanings will threaten the conceptual self.

\subsubsection{Threat to Conceptual Self}

The TCS claims that threat to the conceptual self can result in the development and maintenance of PTSD. Ehlers and Clark (2000) theorize that PTSD is the result of some individuals processing "the traumatic event and/or its sequelae in a way which produces a sense of a serious current threat" (p. 320). They espouse that perceived current threat can be "either external (e.g., the world is a more dangerous place) or, very commonly, internal (e.g., a threat to one's view of oneself as a capable/acceptable person who will be able to achieve important life goals)" (p. 320). Their model predicts that once the perception of current threat is activated it is accompanied by PTSD symptoms. They suggest that PTSD can be exacerbated and maintained by the perceived threat motivating a series of behavioral and cognitive responses that are aimed at reducing perceived threat and distress in the short-term, but have the result of preventing cognitive change and therefore, maintain PTSD.

The TCS claims that threat to conceptual self is analogous to Ehlers and Clark's conceptualization of internal threat. Specifically, threat to conceptual self is a threat to one's view of oneself as a capable/acceptable person who is able to achieve important life goals and/or a threat to the perceived or actual social view of self as a capable/acceptable person who will be able to achieve important life goals. Therefore, the TCS proposes that threat to conceptual self results in the processing of the traumatic event and/or its sequelae in a way which produces a sense of a serious 
current threat and once the perception of current threat is activated, it is accompanied by PTSD symptoms.

Additionally, Sato (2001) suggests that the conceptual self is essential in maintaining mental health. Theorists (e.g., Mesquita \&Walker, 2003; Sato, 2001) argue that those with an independent conceptual self require personal control over the environment, autonomy and a sense of asserting the self to maintain mental health. Thus, situations, events and life encounters that result in actual or perceived reductions in autonomy and personal control over the situation can result in poor psychological adjustment. Sato claims independent cultures expect a strong sense of autonomy in a person allowing them to function properly as a member of society. A person with a strong sense of relatedness, and/or a person lacking autonomy, are seen as "dysfunctional" and are more prone to mental health concerns. In contrast, theorists (e.g., Sato, 2001) argue that those with an interdependent conceptual self require interdependence with others and a sense of fulfilling social obligations, fitting in and maintaining group harmony to maintain mental health. Thus, situations, events and life encounters concerning negative relationships with others, rejection by significant others and a lack of social support can result in poor psychological adjustment. Sato claims interdependent cultures expect a strong sense of relatedness in a person, allowing them to function properly as a member of society; a person with a strong sense of autonomy, and/or a person lacking relatedness is seen as "dysfunctional" and more prone to mental health concerns.

The TCS posits that the conceptual self is essential in maintaining mental health. It proposes that threat to conceptual self can be direct (i.e., a lack of development, expression and maintenance of the culturally dominant self) or indirect (i.e., development, expression and maintenance of the culturally inappropriate self). For those with an independent conceptual self, direct threat to the conceptual self can occur when personal control over the environment and autonomy has diminished. Indirect threat to the conceptual self can occur when there is an increased emphasis on the interdependent self. Increased interdependence suggests the self is less autonomous, less capable and less in control of situations. Additionally, aligned with Sato's claim, the TCS suggests independent cultures will be less tolerant of a trauma survivor with a lessened sense of autonomy, impairing the survivor's ability to function properly as a member of society. This may result in alienation of the trauma survivor. In contrast, the TCS proposes that for those with an interdependent 
conceptual self, direct threat to the conceptual self can occur when either interdependence with others or a sense of fulfilling social obligations, fitting in or maintaining group harmony have diminished. Indirect threat to the conceptual self can occur when there is an increased emphasis on autonomy. Increased self-importance and personal control suggests the self is less interdependent and not maintaining group harmony or fulfilling social obligations. Additionally, it is proposed that interdependent cultures will be less tolerant of a trauma survivor with an increased sense of autonomy or a lessened sense of relatedness, impairing their ability to function properly as a member of society. This is likely to result in alienation of the trauma survivor. Levels of autonomous orientation in the trauma memory pose a direct threat to the independent conceptual self and an indirect threat to the interdependent conceptual self.

In the case of centrality of the trauma memory impacting on the development, expression and maintenance of the conceptual self, threat to the conceptual self is believed to occur as follows. Low levels of autonomous content in autobiographical remembering has the potential of threatening the independent conceptual self, as the self is developed, expressed and maintained in terms of lacking personal control, autonomy, uniqueness, capability, and so forth. High levels of autonomous content in autobiographical remembering has the potential of threatening the interdependent conceptual self as the self is developed, expressed and maintained in terms of increased self-importance, autonomy and personal control. Such self development, expression and maintenance undermine the self as interdependent, maintaining group harmony and fulfilling social obligations. Both instances result in what Ehlers and Clark (2000) term a serious sense of internal threat to self, which is accompanied by PTSD symptoms. Given self-defining memories have been perceived to be a measure of alteration in self-concept (e.g., Sutherland \& Bryant, 2006), support for the proposed impact of the level of autonomous content in the trauma memory on the conceptual self is derived from the finding in Study 3 that those from independent cultures with PTSD had less autonomous orientation in the self-defining memories than did those from independent cultures without PTSD, while those from interdependent cultures with PTSD had more autonomous orientation in their selfdefining memories than did those from interdependent cultures without PTSD.

Centrality of the trauma memory impacting on identity and self-change, which is believed to be moderated by self-consistency needs, threatens the conceptual self as 
it the self is now centered round trauma. Such threat is accompanied by PTSD symptoms, as Berntsen and Rubin (2007) suggest that trauma-centered identity results in current situations being perceived as a serious current threat. The findings (Studies 1 and 3) support this proposal. Those from independent cultures (where selfconsistency is valued) with PTSD were found to have greater trauma-centered selfidentity than did those without PTSD, while there was no difference between those with and without PTSD from interdependent cultures (where self-flexibility is valued) in trauma-centered self-change.

Threat to the conceptual self via appraisals is thought to occur as follows. Low levels of autonomous orientation in the trauma memory are likely to result in negative autonomous appraisals, which impact on the independent conceptual self but have a neutral impact on the interdependent conceptual self. In independent cultures appraisals associated with diminished autonomy and personal control are associated with poor psychological adjustment. In contrast, high levels of autonomous orientation in the trauma memory are likely to result in autonomous appraisals, which have a neutral or positive impact on the independent conceptual self but have limited applicability (Mesquita \& Walker, 2003) or a negative impact on the interdependent conceptual self. High levels of autonomous content in autobiographical remembering may over time result in negative relatedness appraisals, as high levels of autonomy challenge the interdependent self and contradict interdependence, group harmony and adhering to social obligations. Moreover, it is proposed that independent cultures will be less supportive of trauma survivors with a lessened sense of autonomy and interdependent cultures will be less supportive of trauma survivors with a heightened sense of autonomy. This may result in appraisals of social alienation. It is suggested, however, that alienation appraisals culturally differ in their involvement in the development and maintenance of PTSD.

It is proposed that alienation is a maintaining factor in both cultures, but via different means. Ehlers (e.g., Ehlers et al., 2000) suggests that alienation maintains PTSD because appraisals and feelings of alienation result in the PTSD sufferer not engaging with the world. Hence, the survivor fails to alter self-cognitions. This is an individualistic approach. It is proposed that alienation may be maintaining PTSD in interdependent cultures in a different manner. That is, alienation challenges the interdependent self. The survivor feels isolated from the group and thus, perceives them self as failing as a socially valued relatedness being. Consequently, alienation 
impacts on the public (i.e., social roles) and communal (i.e., interpersonal/relatedness) aspects of self. This proposal is supported by Markus and Kitayama's (1991) assertion that for the independent self-construal "others, or the social situation in general, are important, but primarily as standards of reflected appraisal, or as sources that can verify and affirm the inner core of the self" (p. 224) whereas, the interdependent selfconstrual may adhere to goals and motivations that are significantly shaped and dominated by the reaction of others and in doing so gain self-definition. They propose relationships for the independent self-construal may be viewed as a means for obtaining individual goals, whereas in interdependent self-construal, relationships will often be ends in and of themselves.

The findings supported this proposal. Those from independent cultures with PTSD had more negative autonomous appraisals in their trauma memories than did those without PTSD and autonomous appraisals were significant predictors of PTSD. However, those from interdependent cultures with and without PTSD did not differ in terms of negative autonomous appraisals in their trauma memories. Only alienation, a relatedness appraisal, differentiated between those with and without PTSD from interdependent cultures. Alienation in the independent group also distinguished between those with and without PTSD.

The fourth potential outcome threatens the conceptual self as the trauma memory challenges meaning and beliefs systems (transformation of meaning), which are a major component of the conceptual self.

\subsubsection{Maintaining Threat to the Conceptual Self}

As can be seen in Figure 10.1, the TCS model suggests at least two possible maintaining factors of threat to the conceptual self.

First, Wang and Conway (2004) theorize that the conceptual self is used to encode, organize and retrieve autobiographical memories. Therefore, it seems likely that threat to the conceptual self will impact on on-going encoding, organization and retrieval of other autobiographical memories, such as memories of everyday events and self-defining memories. Additionally, autobiographical memories retrieved pretrauma to develop, express and maintain the conceptual self, or re-affirm the culturally sanctioned self, may now be more difficult to retrieve. Therefore, continual autobiographical remembering, which is instrumental in the development, expression and maintenance of the conceptual self, maintains threat to the conceptual self and 
consequently PTSD. This proposal is supported by the findings that demonstrated that those from independent cultures with PTSD not only had less autonomous orientation in their trauma memories than those without PTSD, but also had less autonomous orientation in their autobiographical remembering (everyday events and self-defining memories) in general. In contrast, those from interdependent cultures with PTSD not only had more autonomous orientation in their trauma memories than those without PTSD, but also had more autonomous orientation in their autobiographical remembering (everyday events and self-defining memories) in general.

Second, Ehlers and Clark posit that appraisals centered round the self may maintain PTSD as survivors continue to perceive current situations as threatening and dangerous as they perceive themselves as inadequate and unable to cope in current situations and retrieve information and memories that confirm this inadequacy. Appraisals about others and interpersonal relationships may maintain PTSD because survivors have on-going negative appraisals of interactions with others, and thus, may withdraw from social interactions and are thus, less likely to receive social support and to correct negative beliefs about themselves and others (Ehlers et al., 2000). Additionally, the self may be perceived as isolated and therefore, not maintaining group harmony, fulfilling social obligations and failing in social roles. Trauma survivors retrieve information and memories that confirm this alienation and social role failings. These on-going appraisals of the trauma and its sequelae, and information retrieved from autobiographical memory that aligns with these appraisals, will maintain threat to the conceptual self.

\subsubsection{Summary of Model}

The TCS postulates that in the personal remembering of trauma, despite the universal foregrounding of autonomy, there are individual differences in the level of autonomous orientation. Given, the conceptual self has been found to impact on schema knowledge it is proposed that, based on the conceptual self people have schema-driven expectations as to appropriate levels of autonomous orientation in personal remembering. The level of autonomous orientation in the personal remembering of trauma may violate schema-driven expectations of autonomous orientation in personal remembering. Such deviation from schemata may provide one mechanism by which the trauma memory becomes the cognitive reference point for other autobiographical memories (Berntsen \& Rubin, 2007). This may result in four 
potential outcomes. First, the trauma memory as central in the organization of autobiographical memory impacts on the functional role of autobiographical memory in developing, expressing and maintaining the conceptual self. Second, trauma, depending on self-consistency requirements, may become central to identity. Third, given Ehlers and Clark's proposed reciprocal relationship between trauma memory and appraisals, the centrality of the trauma memory is likely to impact on negative appraisals. Fourth, centrality of the trauma memory is likely to challenge belief systems given autobiographical memory anchors beliefs and values. All four possibilities threaten the conceptual self and thus, current situations may be perceived as a current threat - an internal threat to the self as the self is failing as either the expected independent (e.g., I am incapable, I have no control) or interdependent (e.g., I am socially isolated, I am failing in my social role) self, thus possibly resulting in the development and maintenance of PTSD. This continual threat will be maintained by first, given the self is used to encode, organize and retrieve autobiographical memories (Wang \& Conway, 2004) all other autobiographical remembering will reflect this challenge to the conceptual self. Second, given the conceptual self has been found to impact on the way events are appraised, the threat to the conceptual self will impact on the interpretation and appraisals of current and future situations reaffirming threat to the self.

It is believed that this model is beneficial as it accounts for many of the findings in this thesis. Furthermore, the model is not culturally specific; rather it can be applied to culture, gender and individual differences. Specifically, it suggests both theory and practice consider the dominant conceptual self of the client. It is apparent that further research is needed to further test and develop the model.

\subsection{Clinical Implications}

The socio-cognitive models outlined in this thesis have guided current clinical practice in psychosocial treatment of PTSD. Dalgleish (2004) notes that copious treatment studies indicate that exposure therapy, cognitive therapy, and a combination of the two, are all efficacious in the treatment of PTSD. Most contemporary clinical theories propose that in order to achieve positive outcomes in treating people with PTSD, exposure and cognitive therapy target the following two aspects. First, theories emphasize an elaboration and contextualization of the trauma memory. This is 
thought to involve the development of conceptual associations between separate memories of the trauma event, and an integration of the trauma memory into existing autobiographical memories and current self-knowledge (Conway, 2005; Hembree \& Foa, 2004). Second, theories state that therapy needs target the interpretation and meaning of trauma, the effects of the trauma on lives, and the interpretation and meaning given to symptoms (Dalgleish, 2004). This often involves helping the client make sense of the trauma in respect to aspects of their self-image and goals (Conway, 2005), cognitively re-structuring negative appraisals (Ehlers \& Clark, 2000) and reconciling trauma with prior beliefs (Resick \& Calhoun, 2001).

The results of this thesis clearly indicate that culture moderates the elaboration and contextualization of the trauma memory, the integration of the trauma memory into current self-knowledge, negative appraisals, and belief and meaning systems. Specifically, the findings here suggest that while a clinical awareness of the impact of trauma on factors such as autobiographical memory, identity, self-definition, appraisals and belief systems is important, awareness needs also to consider cultural factors which moderate any impact.

First, in terms of exposure and cognitive work around autobiographical memory, the results (Study 3) indicated that cultural variation in self impacts on the relationship between the nature of the trauma memory and PTSD. It was found that those from independent cultures had significantly less autonomous orientation in their trauma, everyday and self-defining memories than did those from independent culture without PTSD. Such a finding supports Herman's (1992) proposal that treatment needs to focus on empowering the survivor. The finding also supports therapeutic approaches that suggest the "first principle of recovery is the empowerment of the survivor" and that the efforts of "others may offer advice, support, assistance, affection, and care, but not cure" (Herman, 1992, p. 173). Such approaches propose it is not until autonomy has been achieved that therapy should focus on "deepening relationships" with significant others (Herman, 1992, p. 205). That is, these approaches suggest that in independent cultures, therapy needs to focus on increasing autonomous orientation in clients with PTSD. Practically, this may involve exposure work that highlights and focuses on autonomous aspects of the memory. It may also involve clients repeatedly re-telling the trauma event with increased autonomous orientation. 
However, it was found that those from interdependent cultures had significantly more autonomous orientation in their trauma, everyday and self-defining memories than did those from interdependent culture without PTSD. Such a finding suggests that a primary focus on agency and autonomous orientation in interdependent cultures may be less relevant. Furthermore, this finding suggests Herman's (1992) proposals are culturally moderated. Instead the focus of therapy with clients from interdependent cultures may need to center round re-framing and/or reducing the level of autonomous orientation and increasing levels of relatedness. In interdependent cultures this may translate to a downplaying of autonomous aspects of the memory during exposure work and clients re-telling the trauma event with decreased autonomous orientation and/or increased emphasis on relatedness aspects of the memory. Cognitive work may involve cognitive re-structuring of the memory to realign the trauma memory, and possibly everyday and self-defining memories, with the conceptual self.

Second, in terms of cognitive work around the identity and self-definition, the results (Study 1 \& 3) suggest that while a clinical awareness of the impact of trauma on identity and self-definition is important, awareness needs also consider cultural factors. The impact of trauma on identity and self-definition is explored at assessment and addressed in components of cognitive therapy, such as self-schema work. This work aims to address trauma caused "vulnerable identities" such as "the Self as powerless... inferior...nonexistent...futureless" (Brewin, 2003, p. 73), operates to integrate a client's current views of the self (e.g., I am a victim, I am damaged, I have no control) with pre-trauma views and the life story (Conway, 2005), and intends to make sense of the trauma in respect to existing aspects of self-concept and goals (Hembree \& Foa, 2004), to achieve self-consistency.

The thesis found that while trauma-exposed participants from independent cultures with PTSD clearly displayed a more trauma-defined personal identity than those without PTSD, for those from interdependent cultures there were no differences in trauma-centered identity between those with and without PTSD. This finding is suggested to be the result of cultural variation in self-consistency needs and cultural variation in the elements from which self-definition is derived. Namely, interdependent cultures derive self-definition from social roles and emphasize flexibility in the self across social roles. Therefore, the finding indicates that the list of vulnerable "identities", which generally consists of private components of the self, 
needs to be extended to include public and collective components of the self. This would extend to a clinical consideration of social roles, the impact of trauma on such social roles and the value of role complexity and diversity. Moreover, the finding highlights a clinical appreciation that that identity may not always be "vulnerable", especially in interdependent cultures.

Third, in terms of cognitive work around appraisals, this thesis suggests a clinical awareness of the impact of cultural variation in self on the relationship between appraisals and PTSD. The thesis (Studies $2 \& 4$ ) found that while for those from independent cultures, autonomous and relatedness appraisals differentiated between those with and without PTSD, for those from interdependent cultures, only the relatedness appraisal differentiated between those with and without PTSD. It is believed that much of cognitive behavior therapy for the treatment of PTSD focuses on appraisals associated with private aspects of self. It appears, as stated above, even when there is a focus on relatedness appraisals (e.g., alienation) it is approached from an independent self-construal. It is proposed that clinicians need consider appraisals derived from the interdependent aspect of self. This may include exploring and challenging appraisals associated with the public self (e.g., "I am a failure as a mother") and communal self appraisals (such as isolation from the group and a sense of social failing) in assessment and in cognitive work, especially when working with clients from interdependent cultures.

Fourth, the findings (Study 5) suggest cultural variation in self impacts on transformation of meaning. Cultural differences in self were found to impact on an issue that cognitive therapy targets; the interpretation and meaning of trauma memory (Dalgleish, 2004). Thus, during the assessment and therapy stages, clinicians need to consider that those with an independent self-construal may focus on what the trauma has meant to the self and individual life story, whilst for those with an interdependent self-construal assessment may need to focus on what the trauma has meant for their family and other significant groups and communities to which the client belongs, and to the relational aspects of self.

The results suggest that much of cognitive behavior therapy used in the treatment of PTSD reflects Bracken's (2002) proposal that the current clinical awareness remains individualistically focused. The findings of this thesis, however, do not dismiss the usefulness of cognitive behavioral therapy in interdependent cultures. It is suggested that effective components such as imaginal exposure may not 
be as influenced by cultural factors. Additionally, techniques such as goal-setting in life roles using behavioral activation models such as those used for depression (e.g., Lejuez et al., 2001) seem appropriate in the treatment of PTSD in those from interdependent cultures. Moreover, cognitive therapy remains useful in addressing aspects of the trauma experience and appraisals that are not culturally sanctioned. Finally, there is accumulating support (e.g., Schwartz, 2005; Tarrier \& Humphreys, 2003) for the use of interventions that target social support as adjuncts to cognitive behavior therapy in the treatment of PTSD. Practically this suggests an inclusion of a client's interpersonal and social context in case formulation (Tarrier, 2006), an acknowledgment of the impact of the beliefs held by significant others on the development and maintenance of PTSD (Tarrier \& Humphreys, 2003), and the consideration of treatment options that are designed to facilitate and improve social support (Harvey et al., 2003) such as family therapy, re-engagement with social networks, modification of perceptions about the intentions and reactions of others, and a focus on reciprocity and social exchange (Tarrier \& Humphrey, 2003).

The key clinical implication to be drawn from this thesis is that the current focus in cognitive behavior therapy on the private, individualistic, autonomous side of self not be disregarded but rather expanded to include considerations of public, social and communal aspects of the self. It must be noted, however, that this thesis does not promote the stereotyping of clients from particular cultures. Rather, it endorses the continuation of the professionalism of clinical psychology. That is, as upheld in clinical psychology, whoever the client, regardless of gender, ethnicity, sexual orientation, and so forth, a thorough clinical assessment is conducted, a systematic case formulation generated and evidence-supported yet client-focused treatment is employed.

\subsection{Limitations}

The limitations of this thesis will be briefly discussed, given they have been addressed in depth in the studies. The first set of limitations pertains to the method of PTSD diagnosis and the possibility of trauma type moderating the impact of cultural differences in self on trauma responses.

The second set of limitations pertains to the culture variable. First, language and task understanding must be considered. Second, it is possible that cultural 
differences found in autobiographical memory are the result of cultural differences in terms of how cultures express an autobiographical memory, such as appropriate levels of self-disclosure. This "may create a type of 'response bias' in memory narratives rather than actual differences in memory representations" (Wang \& Conway, 2004, p. 930). However, Wang and Ross' (2005) findings indicate cultural differences in autobiographical memory do reflect cultural differences in the way in which people remember autobiographical events rather than simply the way in which they talk or write about these events. Third, it is acknowledged that the independent/interdependent construct is only one cultural dimension and the cultures comprising these groups in this study vary on other cultural dimensions.

The third limitation is related to a theoretical concern. The conceptual framework proposes that trauma memory becomes associated with the activated autonomous goals. Therefore, anytime the goals associated with the trauma memory are activated there is involuntary retrieval of the trauma memory (i.e., re-living symptoms) (Conway, 2005). Hence, the conceptual framework would predict that those from independent cultures would have more re-living symptoms than those from interdependent cultures, given the dominance of autonomous goals in independent culture. There is no clear evidence supporting or disputing this claim. Other models (e.g., appraisal model) suggest strong associative memory and perceptual priming also play a role in re-living symptoms. Further research is required to develop the conceptual framework.

The final set of limitations pertains to methodological issues. First, the hypotheses derived in this thesis were tested using very similar methodology throughout. Thus, the findings may have been a reflection of the methodology, as opposed to actual cultural differences. Consistently across these tasks, however, similar findings emerged. Second, the thesis adopted a universalistic approach to cross-cultural research in that it tested the universality of the existing psychological theories of trauma. The thesis, however, was developed within an individualistic culture and from an independent self-construal and hence its constructs, values, beliefs and methodology may be somewhat biased. However, research is a valueladen activity searching for answers and to by-pass these values completely is near impossible. The hypotheses, however, generated from solid theoretical foundations, were empirically supported. The thesis also involved the opinions of those from 
interdependent cultures by including an Asian coder and discussing the thesis with researchers and clinicians from interdependent cultures.

\subsection{Conclusion}

In conclusion, this thesis investigated cultural differences in self-construal in the autobiographical memory of trauma. The thesis, based on the observation that the socio-cognitive models of PTSD seem to have largely ignored the model of selfconstrual and its associated implications, developed and tested a conceptual framework that transported the PTSD models into the realm of culture via their existing connections with the self. Overall, the conceptual framework was supported.

Despite cultural similarities in the autobiographical remembering of trauma, cultural variation in self was found to impact on the relationship between the autobiographical remembering and psychological adjustment; the influence of trauma on identity and self-definition; trauma and trauma sequelae appraisals and the relationship between appraisals and psychological adjustment; and trauma memory meanings.

In light of these findings, current PTSD models were critiqued in terms of their cultural flexibility in accounting for cultural variation in self-construal. Overall, the findings indicated that although current PTSD models can account for much of the phenomena observed in PTSD, there are important cultural considerations both in the etiology of PTSD and in its maintenance, which are not part of current psychological models of PTSD. The findings challenge these models to articulate how the cultural self aligns with their accounts. It is thought, confirmed by the findings in this thesis, that PTSD models need to be more explicit regarding the impact of the cultural self on the processes involved in development and maintenance of PTSD.

Based on these models and current understandings of the self, the threat to the conceptual self model was developed to account for the findings. Specifically, it provided a working model illustrating the impact of trauma on the conceptual self in the etiology and maintenance of PTSD.

The thesis offered guidelines for clinical practice. It was suggested current elements of cognitive behavior therapy for PTSD focus on the private, individualistic, autonomous side of self. Hence, it was posited that this approach be expanded to include considerations of public/social and communal aspects of the self. 


\subsection{Future Directions}

This thesis initiated an exploration into cultural differences in the autobiographical memory of trauma. However, it is believed to be one of the first studies of its kind and thus, its findings have only scratched the surface. Although clear theoretical and clinical implications emerged, so did many questions and areas requiring further research. Such areas include: a further examination of the threat to conceptual self model and an exploration of cultural differences in the mechanisms involved in the development and maintenance of PTSD. Research is required to explore cultural differences in structural, perceptual, cognitive (e.g., attention, perceptual priming), and biological (e.g., amygdala, hyperarousal responses) processes involved in the development and maintenance of PTSD. Furthermore, cultural differences in self have been found to impact on emotion, hence further research should include an analysis of culture, trauma and emotion. The final study in this thesis indicated that cultural differences in self impact on belief systems and transformation of meaning. However, at this stage our psychological literature is very limited in this respect, and thus further research is needed.

The thesis highlighted a need for future research to employ different methodologies and designs such as prospective studies (i.e., what are the factors that predict the development of PTSD and do these factors differ culturally?), analog studies (e.g., showing participants emotional films and assessing cultural differences in memory), cross-country studies (i.e., trauma survivors in a collectivistic country being compared to trauma survivors in an individualistic country) and studies examining cultural differences in the memory of the same trauma event (e.g., after September 11 or 2004 Indian Ocean Tsunami), to name but a few suggestions.

The thesis also calls for the requirement of cross-cultural treatment studies. An analysis of appraisals and other factors that impact on poorer treatment outcome in interdependent cultures needs to be determined. Additionally, the question remains as to what methods other cultures use in the treatment of trauma responses, whether such methods are efficacious and whether they run adjunct to cognitive behavior therapy. Research is needed to examine whether adding components that focus on communal self and social roles improves the efficacy of cognitive treatment, especially in interdependent cultures. 
Finally, cultural differences in self, is only one cultural dimension. Other cultural factors impact on the trauma memory and its subsequent appraisals needs to be examined. 


\section{REFERENCES}

American Psychiatric Association. (1980). Diagnostic and statistical manual of mental disorders (3rd ed.). Washington DC: Author.

American Psychiatric Association. (1994). Diagnostic and statistical manual of mental disorders ( $4^{\text {th }}$ ed.). Washington DC: Author.

Andrews, B., Brewin, C. R., Rose, S., \& Kirk, M. (2000). Predicting PTSD symptoms in victims of violent crime: The role of shame, anger, and child abuse. Journal of Abnormal Psychology, 109, 69-73.

Annan, K. (2001, December 10). Nobel Lecture. Retrieved November 5, 2007, from http://nobelprize.org/nobel_prizes/peace/laureates/2001/annan-lecture.html

Arrindell, W. A. (2003). Cultural abnormal psychology, Behaviour Research and Therapy, 41, 749-753.

Baddeley, A. (1987). But what the hell is it for? In M. M. Gruneberg, P. E. Morris, \& R. N. Sykes (Eds.), Practical aspects of memory: Current research and issues (pp. 3-18). Chichester, England: Wiley.

Barclay, C. R. (1996). Autobiographical remembering: Narrative constraints on objectified selves. In D. C. Rubin (Ed.), Remembering our past: Studies in autobiographical memory (pp. 94-125). Cambridge: Cambridge University Press.

Bauer, J. J., \& McAdams, D. P. (2004). Personal growth in adult's stories of life transitions. Journal of Personality, 72, 573-602.

Beck, A. T. (1983). Cognitive therapy of depression: New perspectives. In P. J. Clayton \& J. E. Barrett (Eds.), Treatment of depression: old controversies and new approaches (pp. 265290). New York: Raven Press.

Bellah, R. N., Madsen, R., Sullivan, W. M., Swidler, A., \& Tipton, S. M. (1988). Individualism and commitment in American life: Readings on themes of habits of the heart. New York: Harper \& Row.

Berntsen, D., \& Rubin, D. C. (2006). The centrality of event scale: A measure of integrating a trauma into one's identity and its relation to posttraumatic stress disorder symptoms. Behaviour Research and Therapy, 44, 219-231.

Berntsen, D., \& Rubin, D. C. (2007). When trauma becomes a key to identity: Enhanced 
integration of trauma memories predicts posttraumatic stress disorder symptoms. Applied Cognitive Psychology, 21, 417- 431.

Bigner, J. J. (1971). Sibling position and definition of self. Journal of Social Psychology, 84, 307-308.

Blatt, S. J. (1990). Interpersonal relatedness and self-definition: Two personality configurations and their implications for psychopathology and psychotherapy. In J. L. Singer (Ed.), Repression and dissociation: Implications for personality theory, psychopathology, and health (pp. 299-335). Chicago: University of Chicago Press.

Bluck, S., (2003). Autobiographical memory: Exploring its functions in everyday life. Memory, $11,113-123$.

Bluck, S., Alea, N., Habermas, T., \& Rubin, D. (2005). A tale of three functions: The selfreported uses of autobiographical memory. Social Cognition, 23, 91-117.

Bluck, S., \& Levine, L. J. (1998). Reminiscence as autobiographical memory: A catalyst for reminiscence theory development. Ageing and Society, 18, 185-208.

Bochner, S. (1994). Cross-cultural differences in the self-concept: A test of Hofstede's individualism/collectivism distinction. Journal of Cross-Cultural Psychology, 25, 273 283.

Bonanno, G. A., \& Singer, J. L. (1990). Repressive personality style: Theoretical and methodological implications for health and pathology. In J. L. Singer (Ed.), Repression and dissociation (pp. 435-470). Chicago: University of Chicago Press.

Bracken, P. J. (2002). Trauma: Culture, meaning and philosophy. London: Whurr Publishers.

Brewer, W. F. (1986). What is autobiographical memory? In D.C. Rubin (Ed.), Autobiographical memory (pp. 25-49). New York: Cambridge University Press.

Brewer, W. F., \& Treyens, J. C. (1981). Role of schemata in memory for places. Cognitive Psychology, 13, 207-230.

Brewin, C.R. (2003). Posttraumatic stress disorder: Malady or myth? New Haven, CT: Yale University Press.

Brewin, C. R., \& Holmes, E. A. (2003). Psychological theories of posttraumatic stress disorder. Clinical Psychology Review, 23, 339-376.

Briere, J. (1997). Psychological assessment of adult posttraumatic states. Washington, DC: 
American Psychological Association.

Brown, R., \& Kulik, J. (1977). Flashbulb memories. Cognition, 5, 73-99.

Bruner, J. (1990). Acts of meaning. Cambridge, MA: Harvard University Press.

Byrne, C. A., Hyman, I. E., \& Scott, K. L. (2001). Comparisons of memories for traumatic events and other experiences. Applied Cognitive Psychology, 15, 119-133.

Choi, S. H. (1992). Communicative socialization processes: Korea and Canada. In S.

Iwasaki, Y. Kashima, \& K. Leung (Eds.), Innovations in cross-cultural psychology (pp. 103-122). Amsterdam: Swets \& Zeitlinger.

Christianson, S.-A., \& Safer, M. A. (1996). Emotional events and emotions in autobiographical memory. In D.C. Rubin (Ed.), Remembering or past: Studies in autobiographical memory (pp. 218-243). New York: Cambridge University Press.

Cohen, G. (1989). Memory in the real world. Hove, England: Erlbaum.

Cohen, G. (1998). The effects of aging on autobiographical memory. In C. P. Thompson, D. J. Herrmann, D. Bruce, D. J. Read, D. G. Payne, \& M. P. Toglia, (Eds.), Autobiographical memory: Theoretical and applied perspectives (pp. 105-123). Hillsdale, NJ: Lawrence Erlbaum Associates.

Conway, M. A. (1990). Autobiographical memory: An introduction. Buckingham: Open University Press.

Conway, M. A. (1996). Autobiographical knowledge and autobiographical memories. In D. C. Rubin (Ed.), Remembering our past: Studies in autobiographical memory, (67-93). New York: Cambridge University Press.

Conway, M. A. (2005). Memory and the self. Journal of Memory and Language, 53, 594-628.

Conway, M. A., \& Holmes, A. (2004). Psychosocial stages and the accessibility of autobiographical memories across the life cycle. Journal of Personality, 72, 461-480.

Conway, M. A., Meares, K., \& Standart, S. (2004). Images and goals. Memory, 12, 525-531.

Conway, M. A., \& Pleydell-Pearce, C. W. (2000). The construction of autobiographical memories in the self-memory system. Psychological Review, 107, 261-288.

Conway, M. A., \& Rubin, D. C. (1993). The structure of autobiographical memory. In S. F. Collins, S. E. Gathercole, M. A. Conway, \& P. E. Morris (Eds.), Theories of Memory (pp. 103-139). Hillsdale, NJ: Erlbaum. 
Conway, M. A., \& Tacchi, P. C. (1996). Motivated confabulation, Neurocase, 2, 325-339. Conway, M., A., Wang, Q., Hanyu, K., \& Haque, S. (2005). A cross-cultural investigation of autobiographical memory: On the universality and cultural variation of the reminiscence bump. Journal of Cross-Cultural Psychology, 36, 739-749.

Cousins, S. D. (1989). Culture and self-perception in Japan and the United States. Journal of Personality and Social Psychology, 56, 124-131.

Dalgleish, T. (2004). Cognitive approaches to posttraumatic stress disorder: The evolution of multirepresentational theorizing. Psychological Bulletin, 130, 228-260.

Dana, R. H. (Ed.) (2000). Handbook of cross-cultural and multicultural personality assessment. Mahwah, NJ: LEA.

Derogatis, L. R., Lipman, R. S., Rickels, K., \& Cori, L. (1974). The Hopkins Symptom Checklist (HSCL): A self-report symptom inventory. Behavioral Science, 19, 1-15.

Dhawan, N., Roseman, I. J., Naidu, R. K., \&, Rettek, S. I. (1995). Self-concepts across two cultures: India and the United States. Journal of Cross-Cultural Psychology, 26, 606-621.

Draguns, J. G., \& Tanaka-Matsumi, J. (2003). Assessment of psychopathology across and within cultures: Issues and findings. Behaviour Research and Therapy, 41, 755-776.

Dunmore, E., Clark, D. M., \& Ehlers, A. (2001). A prospective investigation of the role of cognitive factors in persistent posttraumatic stress disorder (PTSD) after physical or sexual assault. Behaviour Therapy, and Research, 39, 1063-1084.

Eagle, G. T. (2005). Therapy at the cultural interface: Implications for African cosmology for traumatic stress intervention. Journal of Contemporary Psychotherapy, 35, 199-209.

Ehlers, A., \& Clark, D. M. (2000). A cognitive model of posttraumatic stress disorder. Behaviour Research \& Therapy, 38, 319-345.

Ehlers, A., Clark, D. M., Dunmore, E., Jaycox, L., Meadows, E., \& Foa, E. B. (1998). Predicting response to exposure treatment for PTSD: The role of mental defeat and alienation. Journal of Traumatic Stress, 11, 457-471.

Ehlers, A., Maercker, A., \& Boos, A. (2000). Posttraumatic stress disorder following political imprisonment: The role of mental defeat, alienation, and perceived permanent change. Journal of Abnormal Psychology, 109, 45-55.

Ehlers, A., Mayou, R. A., \& Bryant, B. (1998). Psychological predictors of chronic PTSD after 
motor vehicle accidents. Journal of Abnormal Psychology, 107, 508-519.

Ellsworth, P. C. (1994). Sense, culture, and sensibility. In S. Kitayama, \& H. R. Markus (Eds.), Emotion and culture: Empirical studies of mutual influence. Washington, DC: American Psychological Association.

Emmons, R. A. (1986). Personal strivings: An approach to personality and subjective well-being. Journal of Personality and Social Psychology, 51, 1058-1068.

Feldman Barrett, L., \& Russell, J. A. (1998). Independence and bipolarity in the structure of current affect. Journal of Personality and Social Psychology, 74, 967-984.

Fiske, A. P., Kitayama, S., Markus, H. R., \& Nisbett, R. E. (1998). The cultural matrix of social psychology. In D. T. Gilbert, S. T. Fiske, \& G. Lindzey (Eds.), The handbook of social psychology, (Vol. 4, pp. 915-981). Boston, WA: McGraw Hill.

Fiszman, A., Cabizuca, M., Lanfredi, C., \& Figueira, I. (2005). The cross-cultural adaptation to Portuguese of the Trauma History Questionnaire to identify traumatic experiences. Revista Brasileira de Psiquiatria, 27, 63-66.

Fitzgerald, J. M. (1992). Autobiographical memory and conceptualizations of the self. In M. A. Conway, D. C. Rubin, H. Spinnler, \& W. A. Wagenaar (Eds.), Theoretical perspectives on autobiographical memory, (pp. 99-114). Boston: Kluwer Academic Publishers.

Fivush, R. (1994). Constructing narrative, emotions, and self in parent-child conversations about the past. In U. Neisser \& R. Fivush (Eds.), The remembering self: Construction and accuracy of the self-narrative, (pp. 136-157). New York: Cambridge University Press.

Fivush, R., \& Haden, C. (2003). Introduction: Autobiographical memory, narrative and self. In R. Fivush \& C. A. Haden (Eds.), Autobiographical memory and the construction of a narrative self: Developmental and cultural perspectives (pp. vii-xiv). Mahwah, NJ: Lawrence Erlbaum Associates.

Fivush, R., Haden, C., \& Adam, S. (1995). Structure and coherence of preschoolers' personal narratives over time: Implications for childhood amnesia. Journal of Experimental Child Psychology, 60, 32-56.

Foa, E. B., Cashman, L., Jaycox, L., \& Perry, K. (1997). The validation of a self-report measure for posttraumatic stress disorder: The Posttraumatic Diagnostic Scale. Psychological Assessment, 9, 445-451. 
Foa. E. B., Riggs, D. S., Dancu, C. V., \& Rothbaum, B. O. (1993). Reliability and validity of a brief instrument for assessing post-traumatic stress disorder. Journal of Traumatic Stress, 6, 459-473.

Foa, E. B., Riggs, D. S., Massie, E. D., \& Yarczower, M. (1995). The impact of fear activation and anger on the efficacy of exposure treatment for posttraumatic stress disorder. Behavior Therapy, 26, 487-499.

Freeman, M. (2007). Autobiographical understanding and narrative inquiry. In D. J. Clandinin (Ed.), Handbook of narrative inquiry: Mapping a methodology (pp. 120-145). Thousand Oaks, CA: Sage Publications, Inc.

Garcia, C. B. (2005). Cognitive- behavioral intervention for PTSD in Columbian combat veterans. Universitas Psychologica, 4, 205-219.

Garro, L. C. (2001). The remembered past in a culturally meaningful life: Remembering as cultural, social, and cognitive processes. In C. C. Moore \& H. F. Matthews (Eds.), The psychology of cultural experience (pp. 105-147). Cambridge University Press.

Gergen, K. J. (1968). Personal consistency and the presentation of self. In C. Gordon \& K. J. Gergen (Eds.), The self in social interaction: Classic and contemporary perspectives (Vol. 1, pp. 299-308). New York: Wiley.

Glynn, S., Eth, S., Foy, D. W., Randolph, E. T., Urbaitis, M., Boxer, L., et al. (1999). A test of behaviour family therapy to augment exposure for combat-related posttraumatic stress disorder. Journal of Consulting and Clinical Psychology, 67, 243-251.

Grady, D., \& Lipton, E. (2005, January, 24). After treating victims' bodies, Indonesia and Sri Lanka turn to hearts and minds. New York Times. Retrieved November 2, 2007, from http://www.nytimes.com.

Green, B. L. (1996). Trauma history questionnaire. In B. H. Stamm (Ed.), Measurement of stress, trauma, and adaptation (pp. 366-369). Lutherville, MD: Sidran Press.

Green, E. G. T, Deschamps, J., \& Paez, D. (2005). Variation of individualism and collectivism within and between 20 countries: A typological analysis. Journal of Cross-Cultural Psychology, 36, 321-339.

Greenwald, A. G. (1980). The totalitarian ego: Fabrication and revision of personal history. American Psychologist, 35, 603-618. 
Grey, N., Holmes, E., \& Brewin, C. R. (2001). Peritraumatic emotional "hotspots" in traumatic memory: A case series of patients with posttraumatic stress disorder. Behavioural and Cognitive Psychotherapy, 29, 367-372.

Halligan, S. L., Michael, T., Clark, D. M., \& Ehlers, A. (2003). Posttraumatic stress disorder following assault: The role of cognitive processing, trauma memory, and appraisals. Journal of Consulting and Clinical Psychology, 71, 419-431.

Han, J. J., Leichtman, M. D., \& Wang, Q. (1998). Autobiographical memory in Korean, Chinese, and American children. Developmental Psychology, 34, 701-713.

Harvey, A. G., \& Bryant, R. A. (1999a). A qualitative investigation of the organization of traumatic memories. British Journal of Clinical Psychology, 38, 401-405.

Harvey, A. G., Bryant, R. A., \& Tarrier, N. (2003). Cognitive behaviour therapy for posttraumatic stress disorder. Clinical Psychology Review, 23, 501-522.

Hauer, B. J. A., Wessel, I., \& Merckelbach, H. (2005). Overgeneral memory in depression and posttraumatic stress disorder: The role of intrusive memories. Tijdschrift voor Psychiatrie, 47, 299-308.

Heine, S. J., Lehman, D. R., Markus, H. R., \& Kitayama, S. (1999). Is there a universal need for positive self-regard. Psychological Review, 106(4), 766-794.

Helgeson, V. S. (1994). Relation of agency and communion to well-being: Evidence and potential explanations. Psychological Bulletin, 115, 412-428.

Hembree, E. A., \& Foa, E. B. (2004). Promoting cognitive change in posttraumatic stress disorder. In M. A. Reinecke \& D. A. Clark (Eds.), Cognitive Therapy across the life span. Cambridge, UK: Cambridge University Press.

Herlihy, J., \& Turner, S.W. (2002). Discrepancies in autobiographical memories - implications for the assessment of asylum seekers: Repeated interviews study. British Medical Journal, 324, 324-327.

Herman, J. (1992). Trauma and recovery. The aftermath of violence-from domestic abuse to political terror. New York: Basic Books.

Hermans, D., Van den Broeck, K., Belis, G., Raes, F., Pieters, G., \& Eelen, P. (2004). Trauma and autobiographical memory specificity in depressed inpatients. Behaviour Research and Therapy, 42, 775-789. 
Higgins, E.T. (1996). Knowledge activation: Accessibility, applicability, and salience. In E.T. Higgins \& A.W. Kruglanski (Eds.), Social psychology: Handbook of basic principles (pp.113-168). New York: Guilford Press.

Hinkle, D. E., Wiersma, W., \& Jurs, S.G. (2003). Applied statistics for the behavioural sciences $\left(5^{\text {th }}\right.$ Ed.). Boston, MA: Houghton Mifflin.

Ho, C. N. (1999). The effects of generational status and acculturation on the self-reporting of traumatic experiences and symptoms in a nonclinical Asian population. Dissertation Abstracts International: Section B: The Sciences and Engineering, 59, 5086.

Hofstede, G. (1980). Culture's consequences: International differences in work-related values. Beverly Hills, CA: Sage.

Hofstede, G. \& Hofstede, G. J. (2004). Cultures and organizations: Software of the mind: Intercultural cooperation and its importance for survival. New York: McGraw-Hill. Horowitz, M. J. (1986). Stress response syndromes ( $2^{\text {nd }}$ ed.). Northvale, NJ, Aronson. Horowitz, M. J. (1997). Stress response reactions ( $2^{\text {nd }}$ ed.). Northvale, NJ, Aronson.

Howe, M. L., \& Courage, M. L. (1993). On resolving the enigma of infantile amnesia. Psychological Bulletin, 113, 305-326.

Howe, M. L., \& Courage, M. L. (1997). The emergence and early development of autobiographical memory. Psychological Review, 104, 499-523.

Hsu, F.L.K. (1953). American and Chinese: Two ways of life. New York: Schuman.

Hunt, R. R., \& McDaniel, M. A. (1993). The enigma of organization and distinctiveness. Journal of Memory and Language, 32, 421-445.

Hyman, I. E., \& Neisser, U. (1992). The role of the self in recollections of a seminar. Journal of Narrative and Life History, 2, 81-103.

James, W. (1890). The principles of psychology. Oxford, England: Dover Publications. James, W. (1950). The principles of psychology. Oxford, England: Dover Publications. Janoff-Bulman, R. (1992). Shattered assumptions: Towards a new psychology of trauma. New York: Free Press.

Jaycox, L. H., \& Foa, E. B. (1996). Obstacles in implementing exposure therapy for PTSD: Case discussions and practical solutions. Clinical Psychology and Psychotherapy, 3, 176-184.

Jobson, L. \& O’ Kearney, R. (2006). Cultural differences in autobiographical memory of trauma. 
Clinical Psychologist, 10, 89-98.

Kagitcibasi, C. (1996). Family and human development across cultures: A view from the other side. Hillsdale, NJ: Erlbaum.

Kanagawa, C., Cross, S. E., \& Markus, H. R. (2001). "Who am I?”: The cultural psychology of the conceptual self. Personality and Social Psychology Bulletin, 27, 90-103.

Kelly, G. A. (1955). The psychology of personal constructs. New York, Norton.

Kenardy, J., Smith, A., Spence, S. H., Lilley, P. R., Newcombe, P., Dob, R., \& Robinson, S. (2006). Dissociation in children's trauma narratives: An exploratory investigation. Journal of Anxiety Disorders, 21, 456-466.

Kitayama, S., Markus, H. R., \& Kurokawa, M. (2000). Culture, emotion, and well-being: Good feelings in Japan and the United States. Cognition and Emotion, 14, 93-124.

Kitayama, S., Markus, H. R., Matsumoto, H., \& Norasakkumkit, V. (1997). Individual and collective processes in the construction of self: Self-enhancement in the United States and self-criticism in Japan. Journal of Personality and Social Psychology, 72, 1245-1267.

Kolts, R. L., Robinson, A. M., \& Tracy, J. J. (2004). The relationship of sociotropy and autonomy to posttraumatic cognitions and PTSD symptomatology in trauma survivors. Journal of Clinical Psychology, 60, 53-63.

Kuhn, M. H., \& McPartland, T. S. (1954). An empirical investigation of self-attitudes. American Sociological Review, 19, 68-76.

LeDoux, J. E. (1996). The emotional brain: The mysterious underpinnings of emotional life. New York: Simon \& Schuster.

Lee, A. Y., Aaker, J. L., \& Gardner, W. L. (2000). The pleasures and pains of distinct selfconstruals: The role of interdependence in regulatory focus. Journal of Personality and Social Psychology, 78(6), 1122-1134.

Lee, L. C., \& Zane, N. W. S. (1998). Handbook of Asian American Psychology. Thousand Oaks, CA: Sage.

Lejuez, C. W., Hopko, D. R., \& Hopko, S. D. (2001). A brief behavioral activation treatment for depression. Behavior Modification, 25, 255-286.

Liem, R., Lim, B. A., \& Liem, J. H. (2000). Acculturation and emotion in among Asian Americans. Cultural Diversity and Ethnic Minority Psychology, 6, 13-31. 
Lu, L., Gilmour, R., Ka, S., Weng, T., Hu, C., Cern, J., Huang, S., \& Shih, J. (2001). To ways to achieve happiness: When the East meets the West. Personality and Individual Differences, 30, 1161-1174.

Ma, V., \& Schoeneman, T. J. (1997). Individualism versus collectivism: A comparison of Kenyan and American self-concepts. Basic and Applied Social Psychology, 19, 261-273.

Markus, H. R. (1977). Self-schemata and processing information about the self. Journal of Personality and Social Psychology, 35, 63-78.

Markus, H. R., \& Kitayama, S. (1991). Culture and the self: Implications for cognition, emotion, and motivation. Psychological Review, 98, 224-253.

Markus, H. R., \& Kitayama, S. (1994). A collective fear of the collective: Implications for selves and theories of selves. Personality and Social Psychology Bulletin, 20, 568-579.

Maslow, A. H. (1970). Motivation and personality. ( $2^{\text {nd }}$ ed.) New York: Harper and Row.

Matsumoto, D., Kudoh, T., Scherer, K. R., \& Wallbott, H. (1988). Antecedents of and reactions to emotions in the United States and Japan. Journal of Cross-Cultural Psychology, 19(3), 267-286.

Mauro, R., Sato, K., \& Tucker, J. (1992). The role of appraisal in human emotions: A crosscultural study. Journal of Personality and Social Psychology, 62(2), 301-317.

McAdams, D. P. (1985). Power, intimacy, and the life story: Personological inquiries into identity. Homewood, IL: Dorsey Press.

McAdams, D. P. (2001). The psychology of life stories. Review of General Psychology, 5, 100122.

McAdams, D. P., Bauer, J. J., Sakaeda, A. R., Anyidoho, N. A., Machado, M. A., MagrinoFailla, K., While, K. W., \& Pals, J. L. (2006). Continuity and change in the life story: A longitudinal study of autobiographical memories in emerging adulthood. Journal of Personality, 74, 1371-1400.

McGaugh, J. L. (2003). Memory and emotion: The making of lasting memories. New York: Columbia University Press.

McGuire, W., McGuire, C., Chile, P., \& Fujioka, T. (1978). Salience of ethnicity in the spontaneous self-concept as a function of one's ethnic distinctiveness in the social environment. Journal of Personality and Social Psychology, 36, 511-520. 
McNally, R., Lasko, N., Macklin, M., \& Pitman, R. (1995). Autobiographical memory disturbance in combat-related posttraumatic stress disorder. Behaviour Research and Therapy, 33, 619-630.

Meares, R. (2004). The conversational model: An outline. American Journal of Psychotherapy, $58,51-66$.

Mesquita, B., \& Ellsworth, P. C. (2001). The role of culture in appraisal. In K. R. Scherer, \& A. Schorr (Eds.), Appraisal processes in emotion: Theory, methods, research (pp. 233-248). New York: Oxford University Press.

Mesquita, B., \& Karasawa, M. (2002). Different emotional lives. Cognition and Emotion, 16(1), 127-141.

Mesquita, B., \& Markus, H. R. (2004). Culture and emotion: Models of agency as sources of cultural variation in emotion. In N. H. Frijda, A. S. R. Manstead, \& A. H. Fischer (Eds.), Feelings and emotions: The Amsterdam symposium, Cambridge, MA: Cambridge University Press.

Mesquita, B., \& Walker, R. (2003). Cultural differences in emotions: A context for interpreting emotional experiences. Behaviour Research and Therapy, 41, 777-793.

Miller, P. J., \& Sperry, L. L. (1987). The socialization of anger and aggression. Merrill-Palmer Quarterly, 33, 1-31.

Mouanoutoua, V. L., \& Brown, L. G. (1995). Hopkins Symptom Checklist-25, Hmong version: A screening instrument for psychological distress. Journal of Personality Assessment, 64, 376-383.

Mueser, K. T., Rosenberg, S. D., Fox, L., Salyers, M. P., Ford, J. D., \& Carty, P. (2001). Psychometric evaluation of trauma and posttraumatic stress disorder assessments in persons with severe mental illness. Psychological Assessment, 13, 110-117.

Mullen, M. K., \& Yi, S. (1995). The cultural context of talk about the past: Implications for the development of autobiographical memory. Cognitive Development, 10, 407-419.

Nakamura, H. (1964). Ways of thinking of Eastern peoples: India-China-Tibet-Japan. Honolulu, HI: East-West Center Press.

Neisser, U. (1988). Five kinds of self-knowledge. Philosophical Psychology, 1, 35-59.

Neisser, U. (1994). Self-narratives: True and false. In U. Neisser \& R. Fivush (Eds.), The 
remembering self: Construction and accuracy of the self-narrative (pp. 1-18). New York: Cambridge University Press.

Nelson, K. (1996). Language in cognitive development: The emergence of the mediated mind. New York: Cambridge University Press.

Nelson, K., \& Fivush, R. (2004). The emergence of autobiographical memory: A social cultural developmental theory. Psychological Review, 111, 486-511.

Nisbett, R. E., Peng, K., Choi, I., \& Norenzayan, A. (2001). Culture and systems of thought: Holistic vs analytic cognition. Psychological Review, 108, 291-310.

O’Kearney, R. T., Speyer, J., \& Kenardy, J. (in press). Children's narrative memory for accidents and their posttraumatic distress. Applied Cognitive Psychology.

Oyserman, D., Coon, H., \& Kemmelmeier, M. (2002). Rethinking individualism and collectivism: Evaluation of theoretical assumptions and meta-analyses. Psychological Bulletin, 128, 3-72.

Patel, V., \& Sumathipala, A. (2001). International representation in psychiatric literature: Survey of six leading journals. British Journal of Psychiatry, 178, 406-409.

Paunovic, N., \& Ost, L. (2001). Cognitive-behavior therapy vs. exposure therapy in the treatment of PTSD in refugees. Behaviour Research and Therapy, 39, 1183-1197.

Peng, K., \& Nisbett, R. E. (1999). Culture, dialectics, and reasoning about contradiction. American Psychologist, 54, 741-754.

Pillemer, D. B. (1992). Remembering personal circumstances: A functional analysis. In E. Winograd \& U. Neisser (Eds.), Affect and accuracy in recall: Studies of "flashbulb" memories (Emory symposia in cognition 4th ed., pp. 236-264). New York: Cambridge University Press.

Pillemer, D. B. (1998). Momentous events, vivid memories. Cambridge, MA, Harvard University Press.

Raes, F., Hermans, D., Williams, J. M., Brunfaut, E., Hamelinck, L., \& Eelen, P. (2006).

Reduced autobiographical memory specificity and trauma in major depression: On the importance of post-trauma coping versus mere trauma exposure. In S. M. Sturt (Ed.), New developments in child abuse research (pp. 61-72). Hauppauge, NY: Nova Science Publishers. 
Raes, F., Hermans, D., Williams, J. M., \& Eelen, P. (2005). Autobiographical memory specificity and emotional abuse. British Journal of Clinical Psychology, 44, 133-138.

Resick, P. A., \& Calhoun, K. S. (2001). Posttraumatic stress disorder. In D. H. Barlow (Ed.), Clinical handbook of psychological disorders: A step-by-step treatment manual ( $3^{\text {rd }}$ ed.) (60-113). New York: Guilford Press.

Reviere, S. L. \& Bakeman, R. (2001). The effects of early trauma on autobiographical memory and schematic self-representation. Applied Cognitive Psychology, 15, 89-100.

Reynolds, M., \& Brewin, C. R. (1998). Intrusive cognitions, coping strategies and emotional responses in depression, post traumatic stress disorder and a non-clinical population. Behaviour Research and Therapy, 36, 135-147.

Rhee, E., Uleman, J. S., Lee, H. K., \& Roman, R. J. (1995). Spontaneous self-descriptions and ethnic identities in individualistic and collectivistic cultures. Journal of Personality and Social Psychology, 69, 142-152.

Robinson, J. A. (1986). Autobiographical memory: A historical prologue. In D. Rubin (Ed.), Autobiographical memory (pp. 19-24). Cambridge: Cambridge University Press.

Robinson, J. A., \& Swanson, K. L. (1990). Autobiographical memory: The next phase. Applied Cognitive Psychology, 4, 321-335.

Rogers, T. B., Kuiper, N. A., Kirker, W. S. (1977). Self-reference and encoding of personal information. Journal of Personality and Social Psychology, 35, 677-688.

Ross, M., \& Wilson, A. E. (2000). Constructing and appraising past selves. In D. L. Schacter \& E. Scarry (Eds.), Memory, brain, and belief, (pp. 231-259). Cambridge, MA: Harvard University Press.

Ross, M., Xun, E., \& Wilson, A. E. (2002). Language and the bicultural self. Personality and Social Psychology Bulletin, 28, 1040-1050.

Roth P. L., \& Switzer, F. S. (1995). A Monte Carlo analysis of missing data techniques in HRM setting. Journal of Management, 211, 1003-1023.

Rubin, D. C., \& Kozin, M. (1984). Vivid memories. Cognition, 16, 81-95.

Sato, T. (2001). Autonomy and relatedness in psychopathology and treatment: A cross-cultural formulation. A Genetic, Social, and General Psychology Monographs, 12, 89-127.

Schachtel, E. G. (1947). On memory and childhood amnesia. Psychiatry, 10, 1-26. 
Scherer, K. R. (1984). Emotion as a multicomponent process: a model and some cross-cultural data. In P. R. Shaver (Ed.), Review of personality and social psychology (pp. 37-63). Beverly Hills, CA: Sage.

Scherer, K. R. (1997). The role of culture in emotion-antecedent appraisal. Journal of Personality and Social Psychology, 73, 902-922.

Schwartz, R. S. (2005). Psychotherapy and social support: Unsettling questions. Harvard Review of Psychiatry, 13, 272-279.

Schwartz, B., \& Kim, M. (2002). Honor, dignity, and collective memory: Judging the past in Korea and the United States. In K.A. Cerulo (Ed.), Culture in mind: Toward a sociology of culture and cognition (pp. 209-226). New York, NY: Routledge.

Shallice, T. (1988). From neuropsychology to mental structure. New York: Cambridge University Press.

Singer, J. A., \& Salovey, P. (1993). The remembered self: Emotion and memory in personality. New York, US: Free Press.

Spiro, M. E. (1993). Is the Western conception of the self "peculiar" within the context of world cultures? Ethos, 21, 107-153.

Spitzer, S. P., Couch, C., \& Stratton, J. (1973). The Assessment of the Self. New York: Escort Sernoll Inc.

Spitzer, R. L., Williams, J. B. W., \& Gibbon, M. (1987). Structured Clinical Interview for DSMIII-R (SCID). Biometrics Research Department, New York State Psychiatric Institute.

Stipek, D., Weiner, B., \& Li, K. (1989). Testing some attribution-emotion relations in the People's Republic of China. Journal of Personality and Social Psychology, 56, 109-116.

Stryker, S. (1986). Identity theory: Developments and extensions. In K. Yardley \& T. Honess (Eds.), Self and identity (pp. 89-104). New York: Wiley.

Suh, E.M. (2000). Self, the hyphen between culture and subjective well-being. In E. Diener, \& E.M. Suh (Eds.), Culture and subjective well-being (pp. 63-86). London: MIT Press.

Suh, E.M. (2002). Culture, identity consistency, and subjective well-being. Journal of Personality and Social Psychology, 83, 1378-1391.

Sullivan, H. S. (1953). Interpersonal theory of psychiatry. New York: Norton.

Summerfield, D. (2004). Cross-cultural perspectives on the medicalization of human suffering. 
In G. M. Rossen (Ed.), Posttraumatic Stress Disorder: Issues and Controversies. (pp. 233-246). West Sussex: John Wiley \& Sons.

Sutherland, K., \& Bryant, R. A. (2006). Self-defining memories in posttraumatic stress disorder. British Journal of Clinical Psychology, 44, 591-598.

Tarrier, N. (Ed.). (2006). Case formulation in cognitive behavior therapy: The treatment of challenging and complex cases (pp. 1-11). New York: Routledge/Taylor \& Francis Group.

Tarrier, N., \& Humphreys, A-L. (2003). PTSD and the social support of the interpersonal environment: The development of social cognitive behavior therapy. Journal of Cognitive Psychotherapy, 17, 187-198.

Trafimow, D., Triandis, H. \& Goto, S. (1991). Some tests of the distinction between the private and collective self. Journal of Personality and Social Psychology, 60, 649-655.

Triandis, H. (1989). The self and social behavior in differing social contexts. Psychological Review, 96, 506-520.

Triandis, H. (1995). Individualism and collectivism. Boulder: Westview Press.

Wagar, B. M., \& Cohen, D, (2003). Culture, memory, and the self: An analysis of the personal and collective self in long-term memory. Journal of Experimental Social Psychology, 39, 468-475.

Wang, Q. (2001). Culture effects on adults' earliest childhood recollection and self-description: Implications for the relation between memory and the self. Journal of Personality and Social Psychology, 81, 220-233.

Wang, Q., \& Conway, M.A. (2004). The stories we keep: Autobiographical Memory in American and Chinese Middle-Aged Adults. Journal of Personality, 72, 911-938.

Wang, Q. \& Fivush, R. (2005). Mother-child conversations of emotionally salient events: Exploring the functions of emotional reminiscing in European-American and Chinese families. Social Development, 14, 473-495.

Wang, Q., \& Hsueh, Y. (2000) Parent-child interdependence in Chinese families: Change and continuity. In C. Violato, E. Oddone-Paolucci \& M. Genuis (Eds.), The changing family and child development (pp. 60-69). Aldershot, UK: Ashgate.

Wang, Q., \& Leichtman, M. D. (2000). Same beginnings, different stories: A comparison of 
American and Chinese children's narratives. Child Development, 71, 1329-1346.

Wang, Q., Leichtman, M. D. \& Davies, K. I. (2000). Sharing memories and telling stories: American and Chinese mothers and their 3-year-olds. Memory, 8, 159-178.

Wang, Q., Leichtman, M. D., \& White, S. H. (1998). Childhood memory and self-description: The impact of growing up an only child. Cognition, 69, 73-103.

Wang, Q., \& Ross, M. (2005). What we remember and what we tell: The effects of culture and self-priming on memory representations and narratives. Memory, 13, 594-206.

Watkins, D. \& Gerong, A. (1999). Language of response and the spontaneous self-concept: A test of the cultural accommodation hypothesis. Journal of Cross-Cultural Psychology, 30 , 115-121.

Weiss, D. S., \& Marmar, C. R. (1997). The Impact of Event Scale--Revised. In J.P. Wilson, \& T.M. Keane (Eds.), Assessing psychological trauma and PTSD. (pp. 399-411). New York: Guilford Press.

Wheeler, A. M., Stuss, D. T., \& Tulving, E. (1997). Toward a theory of episodic memory: The frontal lobes and autonoetic consciousness. Psychological Bulletin, 121, 331-354.

Williams, J. M., \& Dritschel, B. H. (1988). Emotional disturbance and the specificity of autobiographical memory. Cognition and Emotion, 2, 437-444.

Williams, J. M. G., \& Scott, J. (1988). Autobiographical memory in depression. Psychological Medicine, 18, 689-695.

Woike, B., Gershkovich, I., Piorkowski, R., \& Polo, M. (1999). The role of motives in the content and structure of autobiographical memory. Personality Processes and Individual Differences, 76, 600-612.

Wu, K. K., \& Chan, S. K. (2004). Psychometric properties of the Chinese version of the Impact of Event Scale-Revised. Hong Kong Journal of Psychiatry, 14, 2-8. 


\section{APPENDIX A}

\section{Individualism Scores}

Table A1

Individualism Score (Hofstede \& Hofstede, 2004) and Group Allocation of Cultures used in Thesis.

Culture Individualism score

Group allocation

Australia

90

Independent

Belgium 70

Independent

Brazil

Interdependent

Burma

Interdependent

Burundi

Interdependent

Cambodia

Interdependent

Canada

80

Independent

China

10

Interdependent

Columbia

Interdependent

Congo

Interdependent

Denmark

70

Independent

India

Interdependent

Indonesia

14

Interdependent

Iran

41

Interdependent 


\begin{tabular}{|c|c|c|}
\hline Culture & Individualism score & Group Allocation \\
\hline Iraq & 38 & Interdependent \\
\hline Japan & 41 & Interdependent \\
\hline Korea & 15 & Interdependent \\
\hline Malaysia & 20 & Interdependent \\
\hline Netherlands & 80 & Independent \\
\hline New Zealand & 80 & Independent \\
\hline Pakistan & 10 & Interdependent \\
\hline Russia & 38 & Interdependent \\
\hline Scotland & 85 & Independent \\
\hline Sierra Leone & 15 & Interdependent \\
\hline Sudan & 27 & Interdependent \\
\hline Thailand & 20 & Interdependent \\
\hline United Kingdom & 85 & Independent \\
\hline United States of America & 91 & Independent \\
\hline Thailand & 20 & Interdependent \\
\hline Vietnam & 20 & Interdependent \\
\hline Zimbabwe & 25 & Interdependent \\
\hline
\end{tabular}




\section{APPENDIX B}

\section{Additional Statistical Analyses for Study 4}

\section{Mental Defeat}

There was no significant difference between interdependent cultures and independent cultures in mental defeat, $F(1,97)=2.84, p>.05$. Those with PTSD had greater mental defeat than those without, $F(1,97)=8.11, p<.01$. The interaction between culture and PTSD status was significant, $F(1,97)=13.68, p<.01$. Independent PTSD $(M=.62, S D=.90)$ had significantly greater mental defeat than independent without PTSD $(M=-.60, S D=.89), t(55)=$ $4.24, p<.01$. However, interdependent PTSD $(M=-.32, S D=.87)$ did not differ significantly from interdependent no PTSD $(M=-.21, S D=.91), t(47)=.44, p>.05$.

\section{Control Strategies}

There was no significant difference between interdependent culture and independent cultures in control strategies, $F(1,97)=.56, p>.05$. Those with PTSD had significantly less control strategies than those without PTSD, $F(1,97)=5.08, p<.05$. The interaction between culture and PTSD status was significant, $F(1,97)=11.09, p<.01$. Independent PTSD $(M=.92$, $S D=.84)$ had significantly greater mental defeat than independent without PTSD $(M=2.03, S D$ $=1.00), t(55)=4.24, p<.01$. However, interdependent PTSD $(M=1.36, S D=1.15)$ did not differ significantly from interdependent no PTSD $(M=1.12, S D=1.13), t(47)=.95, p>.05$.

\section{Alienation}

There was no significant difference between interdependent cultures $(M=-.32, S D=$ $1.03)$ and independent cultures $(M=-.25, S D=1.08)$ in alienation, $F(1,97)=.12, p>.05$. However, those with PTSD $(M=.10, S D=1.01)$ had significantly more alienation appraisals in their memories than did those without PTSD $(M=-.63, S D=.99), F(1,97)=11.70, p<.01$. The interaction between culture and PTSD status was not significant, $F(1,97)=.01, p>.05$. 


\section{Permanent Change}

The difference between interdependent culture and independent cultures in permanent change was not significant, $F(1,97)=3.15, p=.08$. Those with PTSD had significantly more permanent change appraisals than those without PTSD, $F(1,97)=23.51, p<.01$. The interaction between culture and PTSD status was significant, $F(1,97)=10.26, p<.01$. Independent PTSD $(M=2.35, S D=1.16)$ had significantly greater permanent change than independent without PTSD $(M=.57, S D=.63), t(55)=5.21, p<.01$. Furthermore, interdependent PTSD $(M=1.30, S D=1.11)$ did not differ significantly from interdependent no $\operatorname{PTSD}(M=.88, S D=.88), t(47)=1.27, p>.05$.

\section{Simultaneous Regression Analyses}

Independent simultaneous regressions were conducted to examine the variables that predict PTSD in the independent and interdependent groups. For the independent culture group, interpersonal trauma classification was forced into the equation in the first step. Interpersonal trauma explained 23\% $\left(R^{2}\right)$ of the variance for PTSD (multiple $R=.48$, adjusted $R^{2}=.21, F[1$, $56]=16.21, p<.01)$. In the second step the variables mental defeat, control strategies, alienation and permanent were entered simultaneously. This led to a significant increase in the variance explained $\left(R^{2}\right.$ change $\left.=.42, F[4,51]=15.07, p<.01\right)$. On the basis of interpersonal trauma classification and the four cognitive variables, $65 \%$ of the variance in PTSD could be explained (multiple $R=.80$, adjusted $\left.R^{2}=.61, F[5,51]=18.62, p<.01\right)$. Control strategies $(B=.16, S E B$ $=.05, \beta=.34, t=3.12, p<.01)$ and permanent change $(B=-.21, S E B=.05, \beta=-.54, t=4.70, p$ $<.01)$ explained unique variance. For the interdependent culture group, interpersonal trauma classification was forced into the equation in the first step. Interpersonal trauma explained $10 \%$ $\left(R^{2}\right)$ of the variance for PTSD (multiple $R=.02$, adjusted $R^{2}=-.02, F[1,48]=.03, p=.87$ ). In

the second step the variables mental defeat, control strategies, alienation and permanent were entered simultaneously. This did not lead to a significant increase in the variance explained $\left(R^{2}\right.$ change $=.15, F[4,43]=1.93, p=.12)$. On the basis of interpersonal trauma classification and the four cognitive variables, $15 \%$ of the variance in PTSD could be explained (multiple $R=.39$, adjusted $\left.R^{2}=.05, F[5,48]=1.55, p=.20\right)$. Alienation $(B=-.16, S E B=.07, \beta=-.31, t=2.19, p$ $=.03$ ) explained unique variance. 